\title{
From vision to action: \\ Hand representations in macaque grasping areas AIP, F5, and M1
}

\section{Dissertation}

\author{
for the award of the degree \\ "Doctor rerum naturalium" \\ of Georg-August-Universität Göttingen
}

submitted by

Stefan Schaffelhofer

from Linz, Austria

Göttingen, 2014 
Doctoral thesis committee: Prof. Dr. Hansjörg Scherberger (First referee, advisor) Research Group Neurobiology German Primate Center $\mathrm{GmbH}$ Kellnerweg 4 37075 Göttingen

Prof. Dr. Stefan Treue (Second referee) Cognitive Neuroscience Laboratory German Primate Center GmbH

Kellnerweg 4

37077 Göttingen

Prof. Dr. Alexander Gail

Cognitive Neuroscience Laboratory

German Primate Center $\mathrm{GmbH}$

Kellnerweg 4

37077 Göttingen

Prof. Dr. Tobias Moser

Abtl. Hals-Nasen-Ohrenheilkunde

Universitätsmedizin Göttingen

Robert-Koch-Str. 40

37075 Göttingen

External examiners:

Prof. Dr. Hannelore Ehrenreich

Max-Planck-Institut für experimentelle Medizin

Klinische Neurowissenschaften

Hermann-Rein-Str. 3

37075 Göttingen

Dr. Peter Dechent

Universitätsmedizin Göttingen

Abt. Kognitive Neurologie

Robert-Koch-Str. 40

37075 Göttingen

Date of oral examination: $\quad 29^{\text {th }}$ of July 2014 
Herewith I declare that I have written this thesis independently and with no other aids and sources than quoted.

Göttingen, $16^{\text {th }}$ of December 2014

Stefan Schaffelhofer 
To my son Jakob 


\section{Acknowledgements}

First of all, I am grateful to Hansjörg Scherberger for giving me the opportunity to work in his laboratory, giving me all the freedom and confidence needed to work creative and autonomously. I did not only profit from the excellent laboratory conditions, but also from his outstanding experience and knowledge. My gratefulness also belongs to my thesis committee members Stefan Treue, Alexander Gail, and Tobias Moser, who actively supported my thesis projects with their excellent knowledge and advice.

I deeply thank my teachers Johann Rauchenecker and Thomas Haslwanter who influenced my professional career significantly. Their dedication and support sparked my enthusiasm for engineering and science.

I would also like to thank all my colleagues from the Neurobiology Lab. First of all I am grateful for the help of Matthias Dörge, who helped me building the experimental setup and supported me with his valuable advice throughout my thesis. He was always there for me, especially in difficult stages of my dissertation. My warm thanks goes to Natalize Nazarenus, Ricarda Ahlert and Leonore Burchardt for their priceless support in animal training. I thank Andres Agudelo-Toro, Jonathan Michaels, Benjamin Dann, Katharina Menz, Rijk in't Veld, Sebastian Lehmann, Wei-An Sheng, and Jeroen Buil for their advantageous collaborations and fruitful discussions.

My gratefulness also goes to Massimo Sartori and Dario Farina. With their help, a musculoskeletal model was developed that could be applied to the electrophysiological studies included in this thesis. Their experience and effort helped me improving my dissertation.

A deep thank goes to Benjamin Lamplmair who invested many hours in drawing the illustrations included to most manuscripts of this dissertation. 
My practical students Alexander Kratzenberg and Anna-Elisa Roser did not only support me in data collection, but also helped me improving my teaching skills by asking fiddly questions and giving valuable feedback.

My appreciation also goes to the PhD Program of the Center for Systems Neuroscience and the GGNB, providing financial and educational support during my dissertation. Their travel grants allowed me to visit exciting conferences all over the world and to meet outstanding scientist from my field. I am grateful for being member of their graduate program.

Not to forget, I am deeply thankful to my monkeys, Zara and Moe. From my perspective it's a privilege to work with such highly intelligent animals and I am aware of the sacrifice they made.

My final and warmest thanks go to family. Lisa, I can't express in words how grateful I am for your never-ending support, understanding and love. You were always there, especially, when I needed you most. Last but not least, I thank my parents and my sister Birgit, who always believed in me. 



\section{Content}

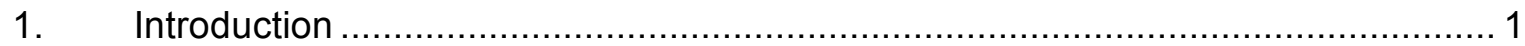

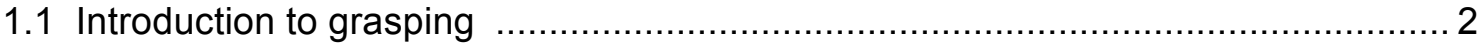

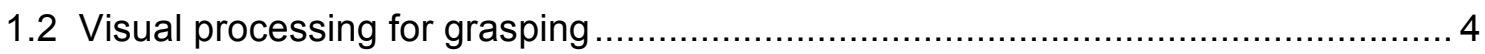

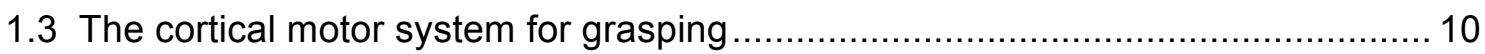

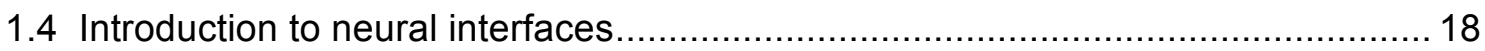

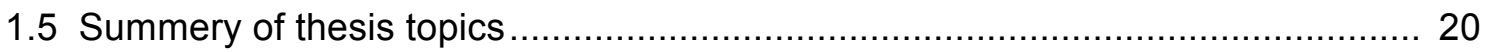

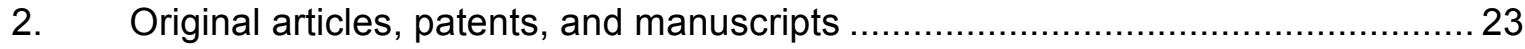

2.1 Tracking of finger, hand and arm movements ..................................... 27

2.2 Musculoskeletal representation of a large repertoire of hand grasping

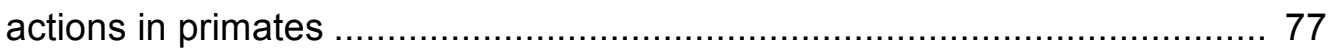

2.3 From vision to action: a comparative population study of hand grasping areas AIP, F5, and M1

2.4 Decoding a wide range of hand configurations from macaque motor,

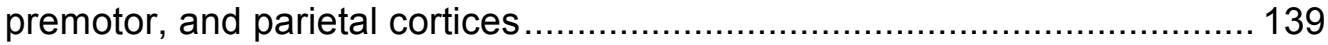

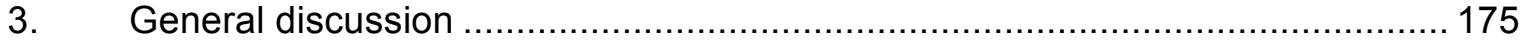

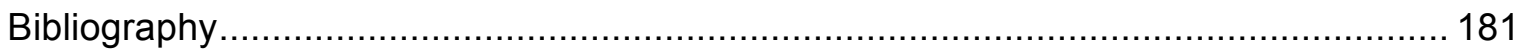

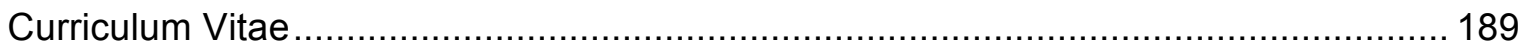





\section{Introduction}

Since the beginning of neuroscience, the investigations of hand movements have been essential for understanding and exploring the motor system. Extensive studies have localized the cortical areas involved in grasping and allowed a first characterization of their electrophysiological attributes. Due to the complexity of the primate hand and its underlying neural mechanisms, essential questions have remained open. This thesis is devoted to further explore the cortical areas involved in hand movement generation and its musculoskeletal system. By utilizing a comprehensive task and novel technologies, light is shed onto the underlying sensory and motor mechanisms.

This thesis comprises four individual studies, focusing on different aspects of grasping in non-human primates. The first study and the resulting patent deal with the tracking of finger, hand, and arm-kinematics. This development was essential for correlating the grasp-kinematics with electrophysiological signals of the brain. The second study presents a three-dimensional musculoskeletal model of the primate upper extremity that was developed for describing motions in the muscle- and the joint angle domain. The third study explores the representation of hand movements in the neocortex and focuses on the visuomotor transformation, a neural process required for translating the visual properties of objects into motor commands. The final work demonstrates that neural planning and motor signals from the cortex can be used to decode a wide range of hand configurations, highlighting their importance for neuroprosthetic control.

This chapter gives an introduction to the physiology of hand movement generation, focusing first on the visual processing of objects and its relevance for grasping. Then, an overview of the hand's motor system is given, including the cortical network and its underlying musculoskeletal system. Chapter 2 constitutes the main part of this thesis, including three original articles, an international patent, and one prepared manuscripts. The final chapter summarizes the results of this thesis and embeds it in a general context. 


\subsection{Introduction to grasping}

Grasping and manipulating objects of different shapes is a fundamental feature of the primate hand. Its sophisticated biomechanics allows us to form a wide range of dexterous hand configurations ranging from precision to power grips (Napier, 1956). In this context, Sir Isaac Newton once said: "In the absence of any other proof, the thumb alone would convince me of God's existence" (Craig, 1867). He realized early that the human hand and the opposition of the thumb is an essential instrument. It allows humans to interact with and construct our environment according to our needs. He believed that without this crucial tool "the most fertile and the most brilliant mind would only be a gift without worth" (Craig, 1867). Forming our hands according to a variety of objects however, requires more than just the advanced biomechanics of our upper extremities. It is a complex process that integrates sensory, motor, and perceptual parameters (Scott, 2004, Castiello, 2005, Brochier and Umilta, 2007).

In order to plan a corresponding hand configuration that matches an object, its shape, size, orientation as well as its meaning have to be processed. The example of Figure 1 summarizes the physiology of visually guided grasping and shows a daily life situation when grasping for a cup of coffee. As a first step, the projection of the cup on the retina starts several visual processes that extract the location of the target along with its shape and orientation (Murata et al., 2000). This information is accessed from pre-motor areas of the frontal lobe that creates motor plans in order to reach for and grasp the target. Additionally, context information of the task is processed to form an appropriate grip (Baumann et al., 2009, Fluet et al., 2010). As an example, we would avoid directly gripping the cup of coffee around the body, because we see and remember it is hot. Instead we would grasp for the handle, thereby applying a different grip (i.e. hook-grip). Once the decision is made, premotor (Borra et al., 2010) and motor areas (Rathelot and Strick, 2009) communicate directly to the spinal cord where alpha motor neurons carry the motor commands to the corresponding muscles of the upper extremity. When the object is touched, sensory receptors on the fingers provide essential feedback about the grip-force applied to the object, 
which is returned to the sensory cortex via the spinal cord. In this control loop, the basal ganglia and the cerebellum play an important role in the judgment of grasp force, and in the correction of error movements, respectively (see also Scott, 2004 for a review). This introduction will focus on two parts of this control loop. At first an overview about the visual processing of object information in the cortex is given. Then, the cortical motor system and its capability of translating visual features into motor commands are discussed.

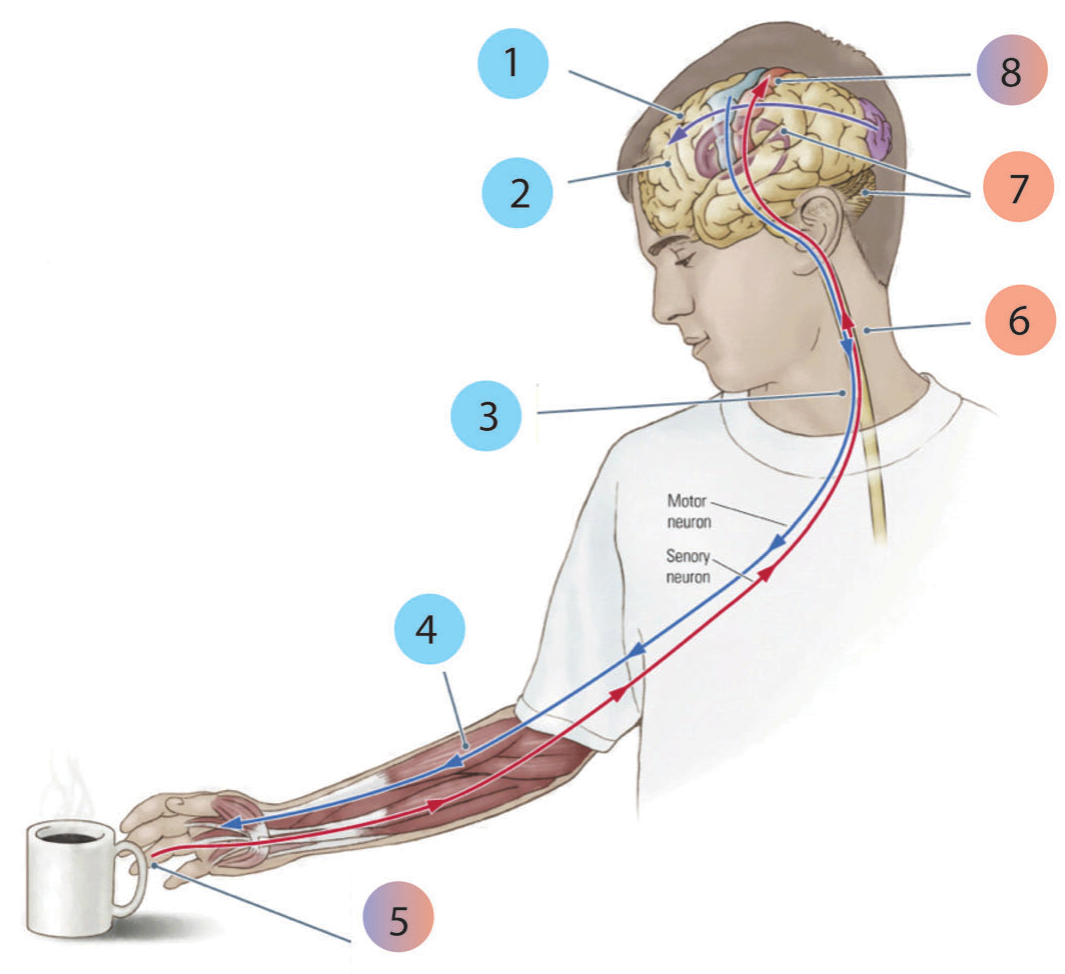

Figure 1 From vision to action. Grasping an object requires the integration of sensory, motor and perceptual parameters: (1) Visual information of an object is extracted and forwarded to the ventral premotor cortex (2) to plan reach and grasping movements. The actual execution is performed together with the primary motor cortex that projects to the spinal cord (3). There, alpha-motor neurons carry the message to the muscles of the hand and forearm (4). When touching the object, sensory receptors on the fingers report information about the applied forces via the spinal cord (6). At the level of the brainstem, cerebellum, and the basal ganglia, forces are judged and movement errors are corrected (7). Finally, the sensory cortex processes the somatosensory information received from the periphery. Adapted from Kolb and Whishaw (2002). 


\subsection{Visual processing for grasping}

The recognition of objects in daily life appears to be trivially simple. We have the capability to distinguish between thousands of objects (Biederman, 1987) and this within a fraction of a second (Thorpe et al., 1996). Although such operations seem to be effortless, their underlying computational mechanisms are highly extensive. This is demonstrated by the fact that half of the neocortex is involved in visual processing (Felleman and Van Essen, 1991, DiCarlo et al., 2012).

If we open a toolbox two aspects of object recognition can be described. On the one hand, we need to have the capability of differentiating shapes and items (i.e. tools) in order to create links to our experience and memories. As an example, recognizing a hammer or a saw as such, allows one to associate the item to its purpose. On the other hand grasping an object requires also considering the physical aspects of an object. We need to know the size of the tool, its position, orientation, and dimensions in order to physically interact with it. It is hypothesized that both aspects of object recognition are processed in separate neuronal pathways.

\subsubsection{Two separate pathways for perception and prehension?}

Two important case studies gave evidence that the brain is by nature separating visual processing for perception and prehension (Goodale et al., 1994). Patient RV, who had sustained bilateral lesions of the occipito-parietal cortex, was unable to use visual information to place her fingers correctly on irregularly shaped objects, whereas she could discriminate such objects from each other. In contrast to this, patient DF, who had a bilateral damage in the ventrolateral occipital region, had no difficulty in placing her fingers on appropriate opposition points during grasping, but was unable to visually differentiate such objects (Goodale et al., 1991). These case studies and later fMRI (functional magnetic resonance imaging) experiments in humans (Culham et al., 2003, James et al., 2003) and monkeys (Nelissen and Vanduffel, 2011) supported the theory from Goodale and Milner (1992) of two separate visual pathways for perception and action. They were termed as the ventral and the dorsal stream (see Figure 2). 
Both pathways take their origin in the primary visual cortex (V1). The ventral steam was described as coursing through the occipitotemporal cortex to its anterior temporal targets (e.g. IT). In comparison, the dorsal stream projects from the occipitoparietal cortex to the posterior half of the inferior parietal lobule (e.g area PG, AIP). The lesion studies of the ventral and the dorsal stream lead to their characterization as the "What" and the "Where" pathways, respectively. Functional descriptions of the dorsal stream and its interconnections to premotor areas caused an additional and more appropriate term of the "How"-pathway (Goodale and Milner, 1992).

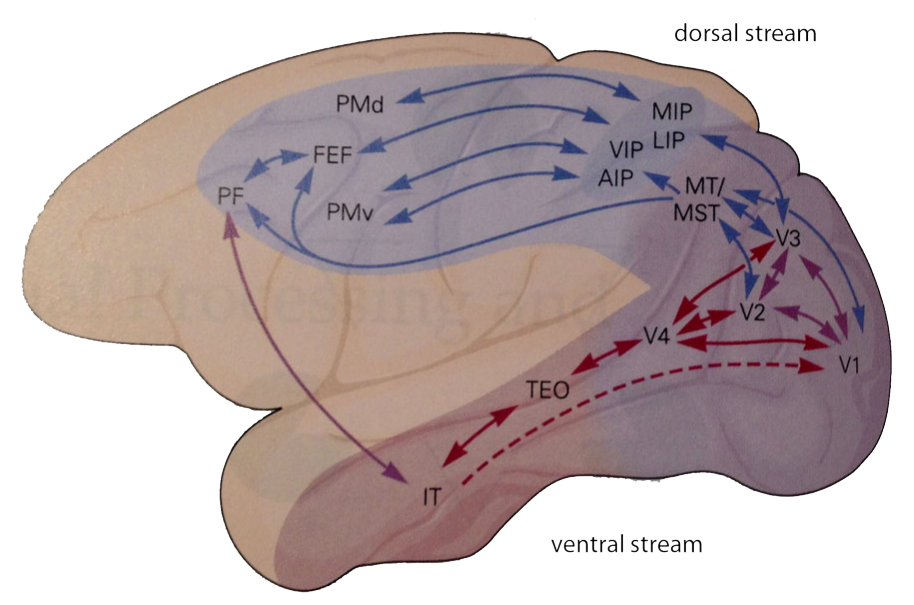

Figure 2. Visual pathways. In the macaque cortex V1 is located at the posterior pole of the occipital cortex and sends axons across two pathways, the ventral and the dorsal stream. The ventral stream projects through V1 to $I T$ and is related to object recognition and perception. In contrast, the dorsal stream courses through the parietal lobe and is linked to the visual processing for movement generation. (AIP, anterior intraparietal cortex; FEF, frontal eye field; IT, inferior temporal cortex; LIP, lateral intraparietal cortex; MIP, medial intraparietal cortex; MST, medial superior temporal cortex; MT, middle temporal cortex; PF, prefrontal cortex; PMd, dorsal premotor cortex; PMv, ventral premtor cortex; TEO, occipitotemporal cortex, VIP, ventral intraparietal cotex; V1, V1, V3, V4, primary, secondary, third, and fourth visual areas).Adapted from Kandel et al. (2000)

A more detailed description was given by Norman (2002), who identified several main differences between the ventral and the dorsal pathway. Most importantly, both streams demonstrate functional differences. Whereas the ventral system is recognizing and identifying the visual input, the primary function of the dorsal system is the analysis of its 
input for visually guided behavior (e.g. reaching and grasping). Secondly, the two streams differ in their sensitivity in spatial and temporal domains. The ventral system is advanced at seeing colors and details of the visual input (Komatsu and Ideura, 1993), while the dorsal stream is better in processing motion (Maunsell and Van Essen, 1983). Subsequently, the dorsal system is faster. This can be explained due to the strong input from magnocellular cells of the retina and the lateral geniculate nucleus (LGN) that were shown to respond faster than parvocellular cells (Merigan and Maunsell, 1990, Merigan et al., 1991). Furthermore, the ventral system requires utilizing long-term memories in order to recognize objects, while the dorsal system depends rather on short-term storage (working memory). Another highly important disparity for grasping is their difference in frame and metric. Both visual systems process information about objects in our environment (Konen and Kastner, 2008) but use them for different purposes (Komatsu and Ideura, 1993, Logothetis et al., 1995, Logothetis and Sheinberg, 1996, Sakata et al., 1997, Murata et al., 2000). The ventral system's functional aim is object identification. For this it needs object-centered information in an allocentric frame of reference. In contrast, the dorsal stream requires information about the location of the object to reach and grasp it. Therefore, it depends essentially on the bodycentered object information in an egocentric frame of reference (Batista et al., 1999, Buneo et al., 2002, Lehmann and Scherberger, 2013).

\subsubsection{Object representation in the ventral and dorsal stream}

Although the ventral and the dorsal stream appear to work autonomously (Goodale et al., 1994, James et al., 2003), they share similar functions. For example, both streams process object shape and sizes (Komatsu and Ideura, 1993, Murata et al., 2000, Fattori et al., 2012, Theys et al., 2012a, Romero et al., 2014) and could synergistically benefit from each other. Evidence of an anatomical connection between the ventral and dorsal stream was given by Borra et al. (2008). They showed that the anterior intraparietal cortex (AIP), known to be involved in hand movement generation, has a strong anatomical linkage to ventral visual stream areas of the lower bank of the superior temporal sulcus. These findings suggest that the information of the ventral stream areas that are involved in object discrimination can be 
directly conveyed to AIP. Getting back to the example of the toolbox, it is not only relevant to process the shape of a saw in motor terms, but also to identify it as a tool (see Palmeri, 2004 for a review of "Object Understanding"). Utilizing this context information allows grasping the saw's handle rather than its toothed edge. In conclusion, both streams are relevant for identifying and grasping objects in healthy subjects. Because of the important role of object processing in grasping, principal neural mechanisms are summarized in this chapter in both the ventral and the dorsal stream.

A

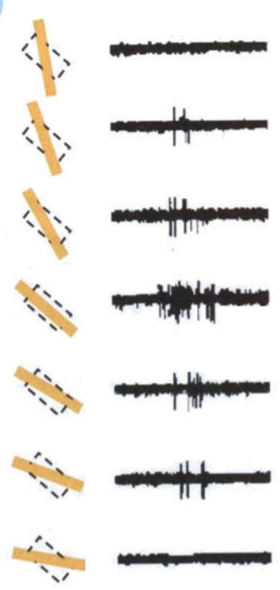

C

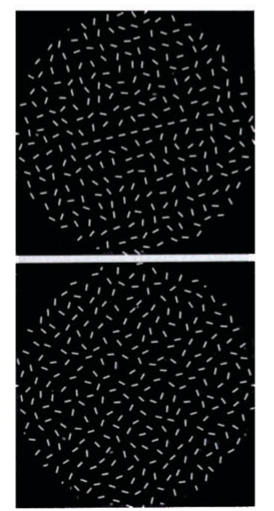

B

$$
\text { Neurons Receptive fields }
$$
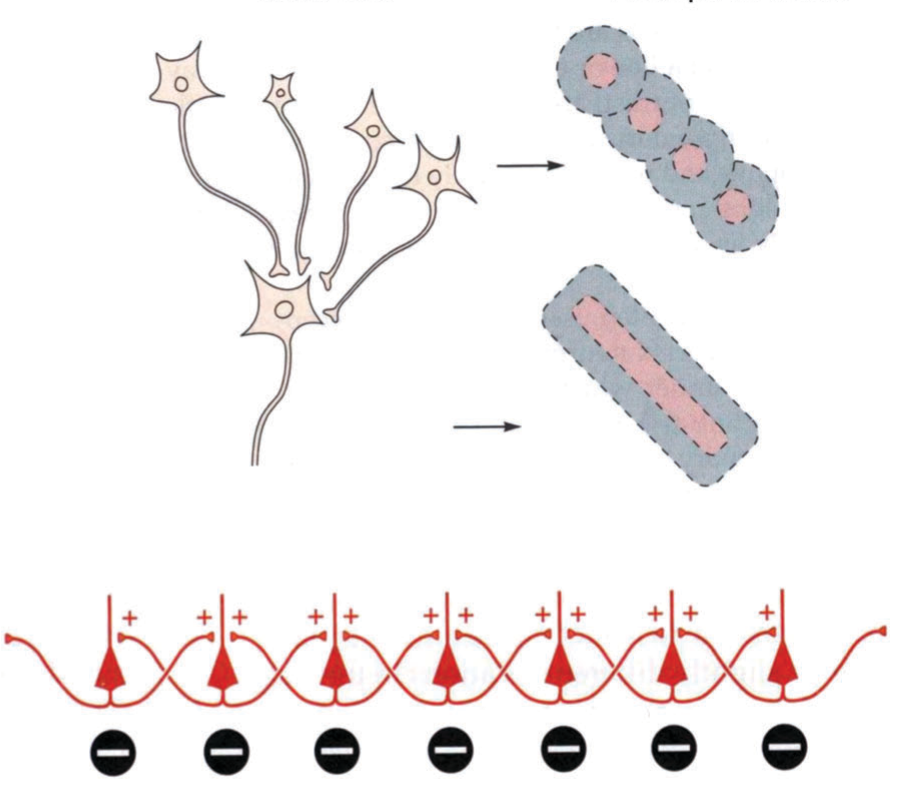

Spacing of collienar line elements

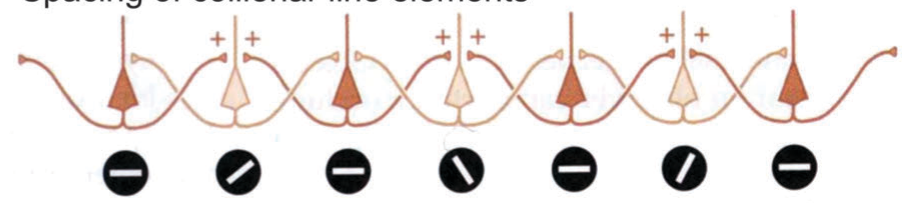

Figure 3. Basic mechanisms of object recognition. (A) "Simple cells" in the primary visual cortex respond selectively to line segments that fit the orientation of the neurons visual field. (B) This kind of response is processed by integrating the information of circular center-surround receptive fields from LGN. (C) Horizontal cells connect the columns of $V 1$ that share the same orientation selectivity. When contour elements are located close to each other in the visual field (first row), excitation can propagate from cell to cell, thereby facilitating the response of V1 neurons. Adapted from Kandel et al. (2000) 
As a first important step in object processing, edges and contours have to be detected. Ganglion cells of the retina and the LGN provide the basis for extracting such features. They have circular receptive fields with center-surround organization and can be subdivided into center-off and center-on cells (Kuffler, 1953). Center-on cells are most activated when the center of the visual field is illuminated whereas center-off cells respond most when the surrounding of their visual field gets illuminated. Therefore, they are detecting the contrast of edges or lines in their visual field but do not respond to their orientation. They can be interpreted as individual pixels of the visual field. In contrast to individual cells of the LGN, the primary visual cortex is able detect orientations (Hubel and Wiesel, 1968) (see Figure 3). The arrangement of ganglion cells in the LGN is highly important for this integration. At this stage, projections are organized as a retinotopic representation of the visual field. This allows the primary visual cortex to create the context between receptive fields located close to each other in the visual field. As shown in the schematic of Figure 3B, center-surround receptive fields of the visual cortex converge to "simple-cells", thereby creating the first representation of orientation. Although the size of visual fields increases along the visual pathway, simple cells cannot detect contours in the global visual scene. Studies in the last decade demonstrated how contextual influences over large regions of visual space could be created in V1 (Li and Gilbert, 2002, Li et al., 2006). Multiple columns of neurons that share the same orientation characteristics (Blasdel, 1992) might be anatomically connected in parallel to the cortex in order to integrate the visual fields of lower level cells, thereby supporting contour detection.

Further integration of contour saliency allows higher visual areas in the ventral stream (Komatsu and Ideura, 1993, DiCarlo et al., 2012) and dorsal stream (Murata et al., 2000, Theys et al., 2012a, Romero et al., 2014) to differentiate individual geometries and objects. Although these processes are not yet fully understood, electrophysiological- and lesion experiments could reveal functional attributes of these areas. Two representative electrophysiological studies of the interconnected areas IT and AIP (Borra et al., 2008) are presented here (see Figure 4). Komatsu and Ideura (1993) visually presented distinct 
shapes to the monkey while recording spiking activity from IT. The results demonstrated that the majority of cells $(62 \%)$ responded to one or more of the stimuli presented in Figure 4 . In analogy to these findings, Murata et al. (2000) showed that more than half of the neurons in AIP responded during object fixation, without a subsequent grasping movement. It was suggested that these "visual neurons" code information about object shape, size and orientation.

Although both areas share major attributes, important differences remain. Whereas IT provided additional information about the color (Komatsu and Ideura, 1993), this feature does not appear in AIP. On the other hand, there is evidence for a retinotopic representation of graspable objects in AIP (Lehmann and Scherberger, 2013), whereas IT shows allocentric, view-independent properties. Furthermore, area AIP demonstrates its strongest connection to the ventral premotor cortex (F5) highlighting its important role in hand movement generation.

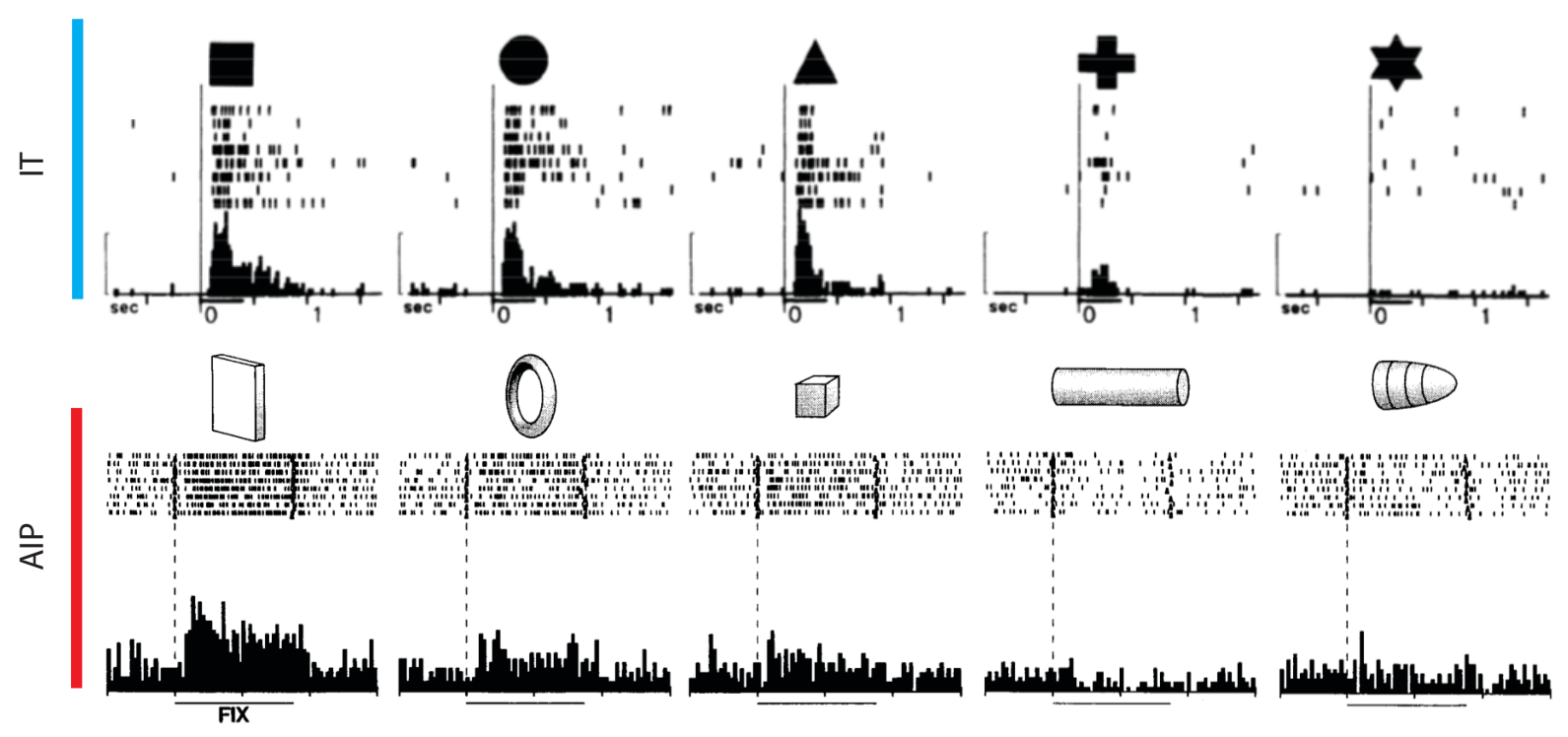

Figure 4. Object processing in ventral and dorsal stream. Peri-stimulus time histograms for two example neurons from IT (first row) and AIP (second raw) are shown, reflecting their spiking activity vs. time. Activity is aligned to cue presentation for both cells. Adapted from Komatsu and Ideura (1993), and Murata et al. (2000). 


\subsection{The cortical motor system for grasping}

The anterior intraparietal (AIP), the ventral premotor (F5), and the primary motor cortex (M1) were identified as the main areas involved in hand movement generation (Rizzolatti and Luppino, 2001, Castiello, 2005). Anatomical studies showed strong interconnections between those areas; Especially AIP and F5, which are part of the fronto-parietal network, revealed intense bidirectional connections (Luppino et al., 1999, Borra et al., 2008, Borra et al., 2010) that are associated with the translation of object properties into motor commands (Murata et al., 2000, Rizzolatti and Luppino, 2001, Raos et al., 2006). Furthermore, F5 and M1 are not only connected to each other, but project to the spinal cord, thereby demonstrating their important role in movement execution (Schmidlin et al., 2008, Borra et al., 2010, Kraskov et al., 2011). This chapter reviews and summarizes functional and electrophysiological attributes of these areas and discusses how the cortical motor system could transform properties of objects into motor signals controlling the musculoskeletal system.

\subsubsection{The anterior intraparietal area (AIP)}

The anterior intraparietal area (AIP) is an end-stage area of the dorsal stream and bridges the gap to the cortical motor areas. Mountcastle and coworkers might have provided the first evidence of grasp-movement modulation in the primate's parietal lobe (Mountcastle et al., 1975). They reported "furious" discharge from a novel group of neurons during grasping and referred to them as "hand-manipulation neurons". The rostral part of the posterior bank of the intraparietal sulcus (IPS) was later designated as AIP (Sakata et al., 1995). Inactivation of this area in non-human primates caused significant impairment of hand functions (Gallese et al., 1994). Specifically, the animals lost their ability to pre-shape their hand to the dimensions of the object while approaching it.

This areas' important role in grasping was further supported by electrophysiological studies, demonstrating grip type (Baumann et al., 2009) and object selectivity (Taira et al., 1990, Sakata et al., 1995, Murata et al., 2000). Based on their specific properties, neurons in AIP were divided into three main classes: "motor-dominant", "visual-dominant", and "visuomotor" neurons (Sakata et al., 1995, Murata et al., 2000). Motor dominant cells become 
activated during grasping and holding in both light and dark. They do not fire during object fixation. Visual-motor neurons discharge stronger during grasping in light than in the dark. Visual-dominant neurons discharge during object fixation and when grasping in light. Interestingly, more than half of these visual cells (visual-dominant and visuo-motor) responded to the presentation of $3 D$ objects (see Figure 4) without the need of a subsequent action, as previously discussed in chapter 1.1.2. In addition, a subgroup of neurons showed size and orientation tuning while grasping and holding the object. The important role for visual processing was further supported recently when AIP neurons were reported to respond selectively to 3D contours (Theys et al., 2012b) and features of shape (Romero et al., 2014).

The variety of neural features highlights the important role of AIP in sensorimotor transformation that is further supported by the rich anatomical connections of AIP to motor, sensory, and higher cognitive areas. Borra et al. (2008) carefully traced the connections from AIP and made several key observations. The most relevant are summarized here. Important for grasping, major reciprocal projections to the ventral premotor cortex (F5) were found in accordance to previous investigations (Luppino et al., 1999). Interestingly, the lateral and the medial part of the inferior arcuate sulcus are more linked to AIP, whereas the medial part showed hardly any connections. Secondly, AIP reciprocally connects to SII, a higher order sensory-motor area, involved in tactile object recognition (Reed et al., 2004). Finally, AIP projects to the ventral stream (TEO, TEa, TEP) known to be involved in object recognition and discrimination (Komatsu and Ideura, 1993, Tanaka, 1996). All of these findings suggest that AIP is highly important for integrating not only visual, but also tactile information for grasping. However, it remains unclear how this variety of features are represented in the neuronal population. More detailed studies have to address the separate visual, motor and proprio-receptive features of AIP-neurons. 


\subsubsection{Area F5}

In the macaque monkey area F5 is located in the rostral part of the ventral premotor cortex (PMv) (Borra et al., 2010). Several investigations associated this area with hand and mouthmovement control (Hepp-Reymond et al., 1994). Especially the posterior bank of the arcuate sulcus (F5ab) seems, for multiple reasons, to be linked to the generation of hand movements. First of all, this area contains many neurons that become activated during natural reach-to-grasp movements (Rizzolatti et al., 1988, Raos et al., 2006, Umilta et al., 2007, Fluet et al., 2010, Lehmann and Scherberger, 2013). Secondly, intracoritcal microstimulations in F5 evoke hand movements and activate distal hand muscles (Godschalk et al., 1995, Schmidlin et al., 2008). Thirdly and similar to AIP, deactivating this area with muscimol leads to strong impairments of the contralateral hand (Fogassi et al., 2001). The pre-shaping of the hand during the reaching phase was most affected. Fourthly, it appears to be one of the main nodes for the transformation of object features into corresponding hand configurations (Jeannerod et al., 1995, Rizzolatti and Luppino, 2001, Davare et al., 2011). This theory is further supported by the areas' anatomical connections. On the one hand, F5ab receives strong input from the anterior intraparietal cortex (Luppino et al., 1999, Borra et al., 2008) that was shown to respond to 3D objects in reach-to-grasp tasks (Murata et al., 2000). On the other hand, it shows vigorous connections to the hand area of motor cortex and, furthermore, also sparse connections to the spinal cord (Dum and Strick, 1991, 2005, Borra et al., 2010).

In contrast to M1, many neurons in F5 are already modulated by grasping action well before the actual movement starts (Raos et al., 2006, Fluet et al., 2010, Townsend et al., 2011, Lehmann and Scherberger, 2013). Different electrophysiological studies described the existence of two types of cells: "motor-neurons", that become active during motor execution and, most importantly for planning, "visuo-motor-neurons", that become active during both object presentation and movement (Murata et al., 1997, Raos et al., 2006). However, the interpretation of these planning-neurons is inconsistent. First Murata et al. (1997) claimed 
that the planning activity is reflecting object information whereas later Raos et al. (2006) suggested the representation of hand shapes/grip types.

In this context it has to be mentioned that changes of object attributes have almost always strong effects on the applied grip. Changes in the discharge rate of a neuron can therefore either be affected by the grip or the object. For this reason, statistical analysis between a small subset of objects as presented so far (Murata et al., 1997, Murata et al., 2000, Raos et al., 2006) can, from my point of view, not provide sufficient evidence for either an object or a grip-type tuning. Differentiating visual and motor features on the neuronal basis would require a more complex object design with a multitude of object and motor representations. Furthermore, additional monitoring of hand kinematics would allow comparing neural patterns with the actual hand configurations. Addressing these questions in a systematic way could therefore be of substantial benefit for understanding the visuomotor processes in the fronto-parietal loop.

\subsection{3 area $\mathrm{M} 1$}

In order to grasp an object, an individual must be able to prepare the movement, pre-shape its hand, and control individual fingers (Rizzolatti and Luppino, 2001). As mentioned above, AIP and F5 are linked to the first part, whereas the latter process depends to a large extent on the hand area of primary motor cortex. Lesions studies in M1 revealed several of its key features such as force deficit, slowness and most importantly, massive impairment of individual finger movements (Schieber and Poliakov, 1998, Fogassi et al., 2001). This fine and independent action control is unique in humans and higher primates and can be explained by the direct connection of cortical motor neurons to alpha-motor neurons of the spinal cord (Rathelot and Strick, 2009). Finding these direct pathways for hand and arm control induced Rathelot and Strick (2009) to subdivide the primary motor cortex into an "old" part and a "new part". The "old" subdivision is standard in many mammals and requires output to go through interneurons of the spinal cord, whereas the "new" subdivision allows direct control of the muscles via alpha-motor neurons. Highly important for 
electrophysiological studies, these dense and direct projections were exclusively found in the rostral part of M1, in the bank of the central sulcus. Furthermore, the connections to distal muscles of the arm, responsible for hand movement control, were significantly more frequent than those linked to its proximal muscles. The complexity of the hand that can be controlled in more than 23 degrees of freedom (DOF) could explain the more numerous connections in comparison to the arm (4 DOF).

Although these tracer studies demonstrated clear evidence for M1's role in movement execution, only some of the neural mechanisms that generate these actions are so far understood. As an example, electrophysiological studies demonstrated a relation to muscle force (Cheney and Fetz, 1980) and joint kinematics (Vargas-Irwin et al., 2010, Aggarwal et al., 2013). Other results suggest a higher-level representation of actions such as movement direction of hand and arm (Georgopoulos et al., 1986, Kakei et al., 1999). In this context, M1 neurons were shown to precisely reflect reach-directions in 3D space (see Figure 5B) (Georgopoulos et al., 1986).

A

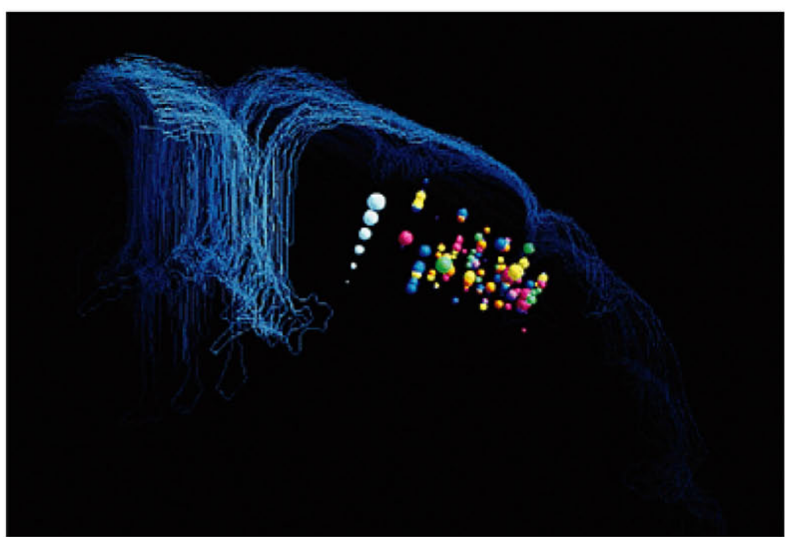

B

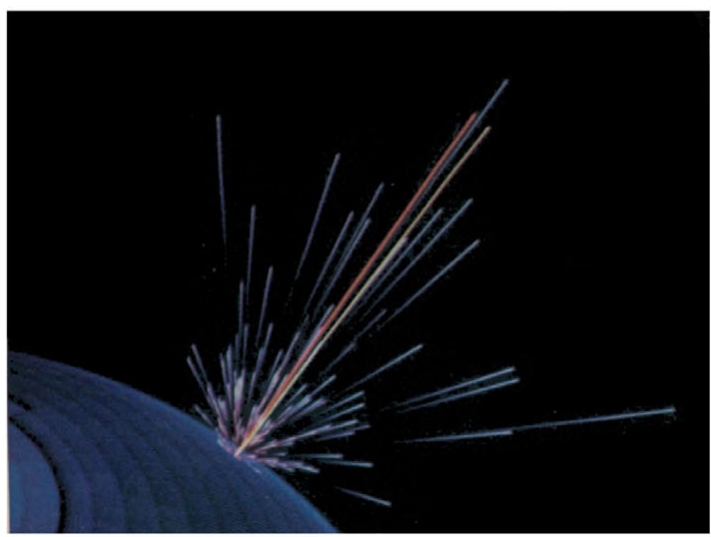

Figure 5. Organization and coding of M1 neurons. (A) Individual finger movements are widely distributed across M1. Each sphere represents a single neuron and its firing rate (size) while flexing or extending its preferred digit (color). Adapted from Schieber and Hibbard (1993). (B) Cortical neurons of M1 reflect reach direction. The image shows the activity of 224 neurons while a rhesus monkey moved into a specific direction in $3 D$ space. The firing rate of each neuron is reflected by the length of vector pointing into the neuron's preferred direction. As shown, the actual movement direction of the arm (yellow) is precisely described by the population activity (orange). Adapted from Georgopoulos et al. (1986). 
Another important question is how movements are organized in primary motor cortex. In the last decades, it became clear that Penfield's classic "homunculus", that suggested a somatotopic representation of the body parts (i.e. individual fingers) can be inaccurate and does not reflect the true nature of primary motor cortex. In contrast to this simplified model, Marc Schieber showed that individual finger movements are widely distributed across M1 (Schieber and Hibbard, 1993, Schieber, 2001) (see Figure 5A). This finding could be explained by the organization of the motor cortex in terms of motor programs that access specific "fractions" of a movement (i.e. individual finger flexion). In this context, stimulation trains of around $500 \mathrm{~ms}$ in M1 evoked coordinated, complex movements that involved many joints (hand-to-mouth, or defending actions) (Graziano et al., 2002, Graziano, 2006). For these reasons, the primary motor cortex can be interpreted functionally as a pre-motor area that stores, activates, and controls motor prototypes that are sent to the the spinal cord.

\subsubsection{Visuomotor transformation}

The neural processes linked to visually guided reach-to-grasp actions begin with an image on the retina and end with coordinated muscle activations. As we have seen, the neural mechanisms in between are complex. Object properties and several coordinate frames have to be considered to reach and grasp for an object in space. One of the key networks that are responsible for transforming visual features into motor commands is the fronto-parietal loop. In this, the parietal reach region (PRR) and the dorsal premotor cortex (PMd) (see Figure 2) were identified to translate eye-centered coordinates of a target into reach plans (Batista et al., 1999, Buneo et al., 2002, Pesaran et al., 2006, Batista et al., 2007), whereas the connection between AIP and F5 was associated with object-hand coordination (Jeannerod et al., 1995, Murata et al., 2000, Rizzolatti and Luppino, 2001, Raos et al., 2006). The areas of the latter control loop were discussed beforehand and are here brought into a general context.

Based on anatomical and electrophysiological results, Giacomo Rizzolatti defined a model that could reflect the interaction of AIP, F5 and M1 in visuomotor processing as shown 
in Figure 6 (Rizzolatti et al., 2001). In this, the visual dominant neurons in AIP create the initial step of transformation (Murata et al., 2000). They extract the 3D object features and subsequently send the relevant information to F5. There, the information coming from AIP activates the visuomotor neurons of F5 (Murata et al., 1997, Raos et al., 2006) that create motor prototypes congruent with the received object description. When activated, these neurons recruit other, purely motor neurons of F5, coding the same motor command. The model further suggests that the selected motor prototype is then executed together with M1.

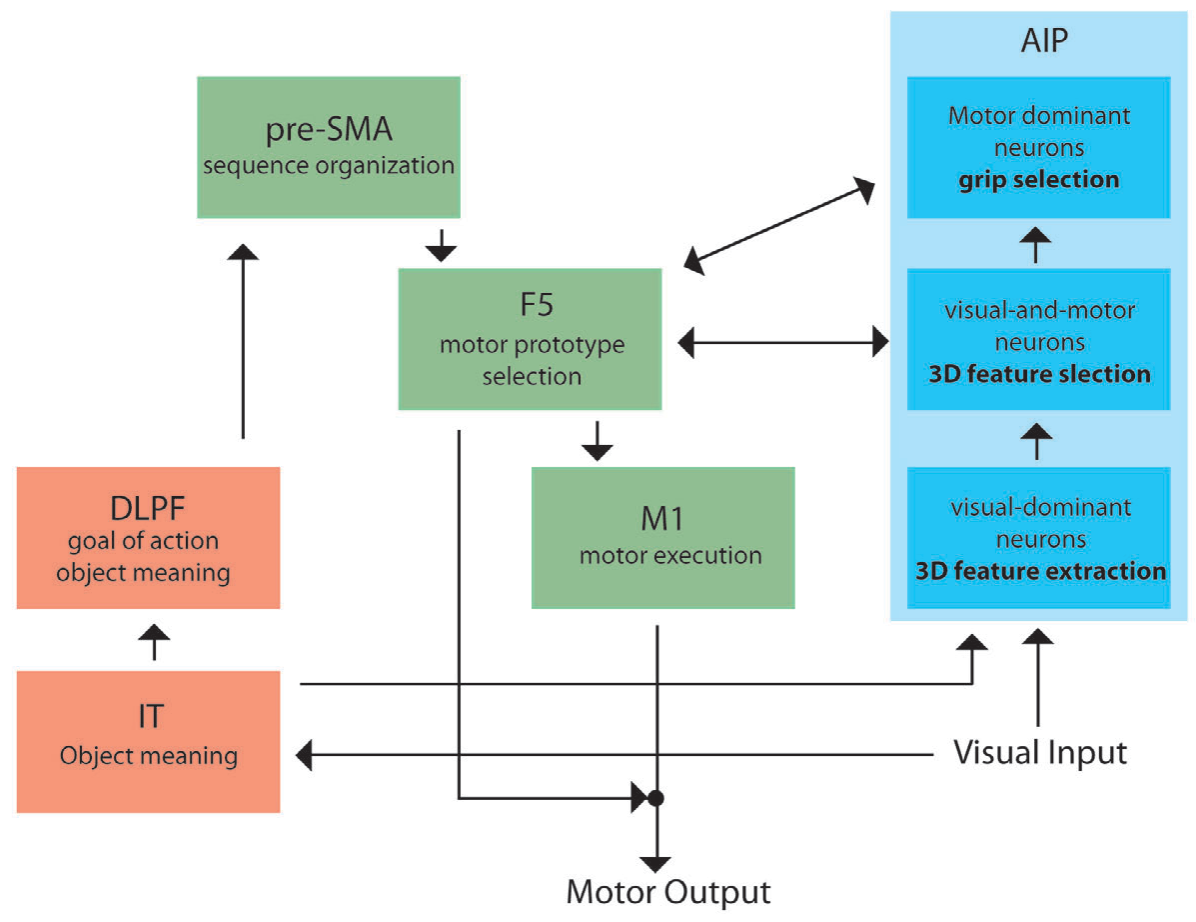

Figure 6. Schematic model of visuomotor transformations for grasping. Visual features of objects are extracted in AIP and forwarded to F5. Context specific information and object identity coded in DLPF and IT support the selection of the corresponding motor command in the ventral premotor cortex that executes the action together with M1. (AIP, anterior intraparietal cortex; F5, ventral premotor cortex; M1, primary motor cortex; IT, inferior temporal cortex; DLPF, dorsolateral prefrontal cortex; SMA, supplementary motor area). Adapted from (Rizzolatti and Luppino, 2001)

However, in most scenarios, an object can offer multiple grasping solutions or in other words: object affordances. Fagg and Arbib (1998) proposed that AIP is not only forwarding one, but multiple presentations of the same object to F5 where different motor 
prototypes become activated. Importantly for this, PMv receives input from the prefrontal cortex and in turn from IT. These connections could provide object identity and higher goals of an action. Based on this information, one of the offered motor commands and object affordances could be selected. In contrast to this theory, the connections of F5 to the prefrontal cortex are only minor interconnected, whereas AIP shows a strong link not only to the prefrontal cortex, but also to IT (Borra et al., 2008). The anatomical connections could therefore suggest a grip selection based on object meaning directly in AIP (Rizzolatti and Luppino, 2001). In this theory, AIP would host multiple object representations, but would only provide one to F5.

Although these models are based on anatomical and electrophysiological studies, the interpretations of visuomotor transformation is so far to a large extend speculative. In order to better understand the processes in the fronto-parietal loop, multi-electrode arrays are required that allow recording from a large population of neurons simultaneously from AIP and F5. Correlating spike traces between different neuron types (e.g. "visual", "visuo-motor") could help better understanding the bidirectional activation of both areas and the extraction of motor commands. 


\subsection{Introduction to neural interfaces}

Paralysis can result from accidents or diseases such as peripheral neuropathies or amyotrophic lateral sclerosis. A disconnection of the body from the spinal cord caused by these disease patterns has fatal influences on the daily life of such patients. The ultimate goal of neural interfaces $(\mathrm{NI})$ is to bridge this emerged gap between the nervous system and the outside world in order to restore parts of the lost functions, such as sensory or motor capabilities (Hatsopoulos and Donoghue, 2009, Scherberger, 2009).

To build NIs, different kind of electrodes can be used to access and decode the signals of the brain (Hochberg and Donoghue, 2006). EEG-based interfaces allow recording non-invasively from surface-electrodes that are attached to the head. Although this kind of signal allowed partially recovering communication and motor functions (Townsend et al., 2010, Diez et al., 2013), its information transfer rate is limited (Hochberg and Donoghue, 2006). In contrast, micro-electrode arrays (MEA) that are implanted in the cortex allow directly recording from hundreds of individual neurons. In this way, cortical implants give access to the direction of movement that is coded accurately by populations of M1 neurons (see chapter 1.3.3) (Georgopoulos et al., 1986). Acquiring such neural information in realtime allowed paralyzed patients to control computer cursers by motor imagination (Kim et al., 2008, Kim et al., 2011). The principle of movement-direction decoding was also applied in non-human primates to control reach velocity of robotic arms in 3D space (Velliste et al., 2008). The know-how acquired from these studies later allowed human subjects to control the position of an artificial arm as well as the orientation and the aperture of a 1-dimensional gripper (Hochberg et al., 2012, Collinger et al., 2013). All of these brain machine interfaces (BMI) rely on instant visual feedback from their assistive devices (e.g. cursor and arm position) and are therefore termed as "close looped" applications. At the moment, several research institutes are working on providing additional sensory information that is measured by the prosthetic device (O'Doherty et al., 2009, Gilja et al., 2011, O'Doherty et al., 2011). In this context, the goal of future neural interfaces is not "only" to receive information from the 
cortex, but also to actively return sensory feedback such as force feedback or the sensation of touch.

Despite the impressive advances of controlling robotic arms, the neural guidance of hand prosthetics remains a major challenge. The reason for the difficulty of such applications can be explained by the complexity of the primate hand (Napier, 1956, Schieber and Santello, 2004). While reaching in space includes three degrees of freedom (DOF), this number increases to 26 DOF or more when considering the joints of wrist and fingers of an anthropomorphic robotic hand. Decoding movement intentions from higher order planning areas could therefore be an alternative approach (Musallam et al., 2004, Subasi et al., 2010, Townsend et al., 2011). Accessing motor commands (i.e, grip types) instead of individual degrees of freedom that form the movement could help reducing the dimensionality problem of such decoding applications (Andersen et al., 2010). The areas of the fronto-parietal loop, including AIP and F5 were demonstrated to be optimal for this kind of task (see chapter 1.3.1 and 1.3.2). Neurons in these areas reflect grip types and wrist orientation during the planning and the execution of hand movements (Baumann et al., 2009, Fluet et al., 2010) and can in turn be successfully decoded (Carpaneto et al., 2011, Townsend et al., 2011, Carpaneto et al., 2012). However, the studies presented so far predicted a small number of most extreme grips (i.e., precision and power grip). Addressing the question of how detailed hand configurations can be decoded from motor planning signals could be highly relevant for the development of neuroprosthetic hands. 


\subsection{Summary of thesis topics}

This thesis explores the neural mechanisms of hand movement generation in the primate's parietal, premotor, and motor cortex.

The detailed study of these areas required the development of a hand tracking device and a 3D musculoskeletal model of the primate upper extremity in order to correlate electrophysiological signals of the brain with the kinematics of the hand. Combining both technologies allowed extracting joint angles and muscle parameters at a high accuracy. These engineering projects are reported in chapters 2.1-2.2.

In chapter 2.3, an additional study investigated the cortical mechanism of hand grasping areas AIP, F5, and M1. Two macaque monkeys were trained to grasp 50 different objects while we recorded from large population of neurons using micro-electrode arrays. The extensive number of conditions caused a high variation of hand shapes and allowed separating visual and motor features at the population level. This study gives new insights how visual information is used to create and execute motor commands.

These findings and the developed technologies were used in chapter 2.4 to demonstrate the decoding of a large number of grip types from cortical planning and execution signals. Additionally, we demonstrated the possibility of translating the decoded hand configurations to an anthropomorphic robotic hand.

Finally, in chapter 3, a comprehensive discussion summarizes the obtained findings of the individual studies in a general context. 




\section{Original articles, patents, and manuscripts}

This chapter contains the following research articles and manuscripts:

\subsection{Tracking of finger, hand and arm movements based on electro-magnetic sensor coils}

The methodology presented in this chapter was applied in small primates and humans and published as a journal article (2.1.1) and a patent (2.1.2), respectively.

\subsubsection{A new method of accurate hand- and arm-tracking for small primates} Schaffelhofer S, Scherberger $H$ (2012) A new method of accurate hand- and armtracking for small primates. Journal of neural engineering. 9:026025.

Author contribution: SS and HS designed the task. SS developed the soft- and hardware of the prototype, implemented the experiment, trained the animal and collected and analysed the data. SS and HS wrote the manuscript.

\subsubsection{Modelling of hand and arm position and orientation}

Scherberger H, Schaffelhofer S (2013) Modelling of hand and arm position and orientation. US Patent 20,130,158,946.

Author contribution: HS and SS equally developed the mathematical model and methods that are part of this patent. SS developed the prototype of the instrumented glove. SS wrote the manuscript. HS edited the manuscript. Kimio Takahashi (Vossius \& Partner, Munich, Germany) converted the manuscript into an international patent.

\subsection{Musculoskeletal representation of a large repertoire of hand grasping actions in primates}

Schaffelhofer S, Sartori M, Scherberger H, Farina D (2014) Musculoskeletal representation of a large repertoire of hand grasping actions in primates. IEEE Trans Neural Syst Rehabil Eng.

Author contribution: SS and HS designed the task. SS trained both animals, implemented the experiment, recorded the data, pre-processed the kinematics, and wrote the interface to the modelling software OpenSim. MS designed the musculoskeletal modelling pipeline that was further developed together with SS. SS 
and MS performed the musculoskeletal analyses and took the lead in writing. HS and DF supervised the project and edited the manuscript.

\subsection{From vision to action: a comparative population study of hand grasping areas AIP, F5, and M1}

Schaffelhofer S, Scherberger H (prepared for submission)

Author contribution: SS and HS designed the task. SS implemented the experiment developed all required tools, trained the animals, recorded and analysed the data and wrote the manuscript. HS edited the manuscript.

\subsection{Decoding a wide range of hand configurations from macaque motor, premotor, and parietal cortex}

Schaffelhofer S, Agudelo-Toro A, Scherberger H. Decoding a wide range of hand configurations from macaque motor, premotor, and parietal cortices. J Neurosci. In press.

Author contribution: SS and HS designed the task. SS built the experimental setup, trained both animals, recorded and analysed the kinematic- and electrophysiological data. AAT and SS developed the translation tool for converting the recorded hand and arm kinematics to a robotic device. SS and AAT wrote the manuscript that was edited by HS. 




\subsection{Tracking of finger, hand and arm movements}

In this chapter a novel instrumented glove for tracking finger, hand and arm movements is presented. The computational model behind the device utilizes the information of electromagnetic sensors that were attached to the hand in order to compute a $3 \mathrm{D}$ model of the upper extremity in real-time. For this, anatomical restrictions and the anthropometry of hand were exploited in order to extract the position of 18 joints and 28 DOF by using only 7 sensors. To our knowledge, this ratio between DOF and number of sensors is higher than any other published or commercially available method. Furthermore, a graphical user interface was developed that enables users to setup the hardware, monitor the kinematics and to transfer the data over several interfaces to electrophysiological recording systems. The methodology was successfully applied and evaluated with prototypes built for nonhuman primates and humans and presented here in chapter 2.1.1 and 2.1.2, respectively. The development of the instrumented glove was essential for this thesis and provided the basis for investigating the kinematics of the primate hand and its correlation to the cortical network.

Follow links to access:

Demonstration video

Journal article (Journal of Neural Engineering)

Patent (US Patent specifications) 


\subsubsection{A new method of accurate hand- and arm- tracking for small primates}

A new hand-tracking method for primates

\section{S Schaffelhofer ${ }^{1}$ and $\mathrm{H}$ Scherberger ${ }^{1,2, *}$}

${ }^{1}$ Deutsches Primatenzentrum GmbH, 37077 Göttingen, Germany

${ }^{2}$ Department of Biology, University of Göttingen, D-37077 Göttingen, Germany

Correspondence should be addressed to Hansjörg Scherberger, Kellnerweg 4, D-37077 Göttingen, Germany. Email: hscherberger@dpz.eu

Acknowledgments: The authors thank Leonore Burchardt and Natalie Nazarenus for assistance in animal training, Matthias Dörge, and Benjamin Wellner for technical assistance, Lisa Sattlegger for glove production, and Tanya Lewis and Katharina Menz for comments on an earlier version of the manuscript. This work was supported by the BMBF (BCCN II, FKZ 01GQ1005C). 


\begin{abstract}
The investigation of grasping movements in cortical motor areas depends heavily on the measurement of hand kinematics. Currently used methods for small primates need either a large number of sensors or provide insufficient accuracy. Here, we present both, a novel glove based on electro-magnetic tracking sensors that can operate at a rate of $100 \mathrm{~Hz}$, and a new modeling method that allows to monitor 27 degrees of freedom (DOF) of the hand and arm using only 7 sensors. A rhesus macaque was trained to wear the glove while performing precision and power grips during a delayed grasping task in the dark without noticeable hindrance. During 5 recording sessions all 27 joint angles and their positions could be tracked reliably. Furthermore, the field generator did not interfere with electrophysiological recordings below $1 \mathrm{kHz}$ and did not affect single cell separation. Measurements with the glove proved to be accurate during static and dynamic testing (absolute error below $2^{\circ}$ and $3^{\circ}$, respectively). This makes the glove a suitable solution for characterizing electrophysiological signals with respect to hand grasping and in particular for brain-machine interface applications.
\end{abstract}

Keywords: Motor, Hand, Arm, Kinematic, Monkey, Grasping, and Reaching 


\section{Introduction}

In the past decade, the investigation of cortical motor areas in human and non-human primates has brought new insights into how the brain encodes grasping movements (Georgopoulos et al., 1986, Schieber and Hibbard, 1993, Lemon, 2008, Baumann et al., 2009, Vargas-Irwin et al., 2010). However, detailed processes in primary-, premotor, and parietal areas and their relationship to hand kinematics are still poorly understood. One reason is the difficulty of tracking hand kinematics of the research field's primary subject, the macaque monkey. So far, grasping tasks have been mainly monitored with sensor-equipped manipulanda (Schieber, 1991, Murata et al., 1997, Murata et al., 2000), and hand kinematic tracker have been developed only very recently (Overduin et al., 2010, Vargas-Irwin et al., 2010). Necessary attributes for hand tracking systems that solve this problem include high sampling rate, high accuracy, compactness and robustness.

Tracking human hand movements has already been realized successfully for more than 20 years. The DataGlove (VPL Research Inc., Redwood City, CA, USA)(Zimmermann and Lanier, 1991) was the first commercially available input glove to gain widespread use in the research community. It was based on optical flex-sensors (Zimmermann, 1985) but has been criticized for being unable to measure finger abduction and adduction (MacKenzie, 1995, Williams, 1997). Current optical gloves, such as the 5DT glove (5DT Inc., Irvine, CA, USA) or the ShapeHand (Measurand Inc. Fredericton, NB, CAN) additionally provide finger abduction/adduction. This results in 14 and 22 degrees of freedom (DOF) of the hand, respectively. Another method for the recording of joint flexion uses electro-resistive sensors (Yun et al., 1997, Simone et al., 2007, Gentner and Classen, 2009) (e.g. CyberGlovell; CyberGlove Systems LCC, San Jose, CA, USA). The resistance of these sensors varies according to the bending of the sensor. Further technology for finger tracking includes Hall-sensors (Dipietro et al., 2003) that is commercially available as HumanGlove (Humanware S.R.L. Pisa, IT) and force resistive sensors that allow additional detection of forces to emphasize haptic perception (Castro and Cliquet, 1997, Yun et al., 1997) (e.g. TouchGlove; Infusion Systems Ltd., Montreal, CAN).

For non-human primates, the first instrumented glove was based on flex sensors (Overduin et al., 2010), which is also the most common technology in humans. The glove tracks 9 DOF with 9 sensors that are placed onto the desired joints. This type of sensor was evaluated earlier by Simone et al. (2005), who found the sensor to be insufficient for sensing the absolute magnitude of bend angles. Also, electro resistive bend sensors are non-linear and therefore require a time consuming calibration for each sensor (Simone et al., 2007, Overduin et al., 2010).

Another recently presented method (Vargas-Irwin et al., 2010, Zhuang et al., 2010) uses 29 optical markers and 12 infrared cameras to track 25 DOF of the hand and arm of a rhesus monkey. Optical systems provide highly accurate positioning (below $1 \mathrm{~mm}$ ), but markers always rely on line-of sight to 
the cameras. Therefore, the grasping of objects that obscure markers, such as handles or objects with cavities, cannot be tracked.

As a solution for these problems, we present a novel data glove for macaque monkeys based on highly accurate electro-magnetic tracking that uses only 7 electro-magnetic sensors to track 27 DOF of the fingers, hand, and arm with a sampling rate of $100 \mathrm{~Hz}$.

\section{Methods}

Animal care and all experimental procedures were conducted in accordance with German and European laws governing animal care and were in agreement with the Guidelines for the Care and Use of Mammals in Neuroscience and Behavioral Research (National Research Council, 2003).

\section{Glove}

The goal of the glove design was to build a custom-fitted, robust device for daily use with macaque monkeys. Figure 1 shows a schematic drawing of the data glove in the context of the experimental setup and on the animal's hand. Our glove consists of three parts: a wristband, a fingerless glove and fingertip caps (figure 1(b)). The glove holds seven magnetic sensors (see below: "Sensor System"), one on each of the five finger caps, one on the hand's dorsum and one on the distal forearm. To ensure tight fitting of the sensors, the finger caps are made of elastic silicon tubes, whereas the fingerless main glove and the wristband are made of spandex (Spandex House Inc., New York, USA). Sensors are held by thin plexiglass plates $(7 \times 4 \times 0.8 \mathrm{~mm})$ that are stitched onto the glove and finger caps. Such a plate avoids possible tilt of the sensors and adapts to the finger shape when stitched onto the elastic silicon tubes. The full glove including sensor cables $(1 \mathrm{~m})$ weighs $16 \mathrm{~g}$. Placement of the glove starts by pulling the elastic glove over the monkey's hand. The glove is adjusted properly and fixed with a Velcro (hook and loop) fastener on the wristband. Then, the elastic fingertip caps are stretched with a sleeving clamp (Facom GmbH, Wuppertal, Germany) and pulled over the animal's fingers.

To achieve high accuracy of fit, a plaster cast model of the monkey's hand was made while the animal was anesthetized for routine examinations. The glove was then fitted to this plaster model. 
(a)

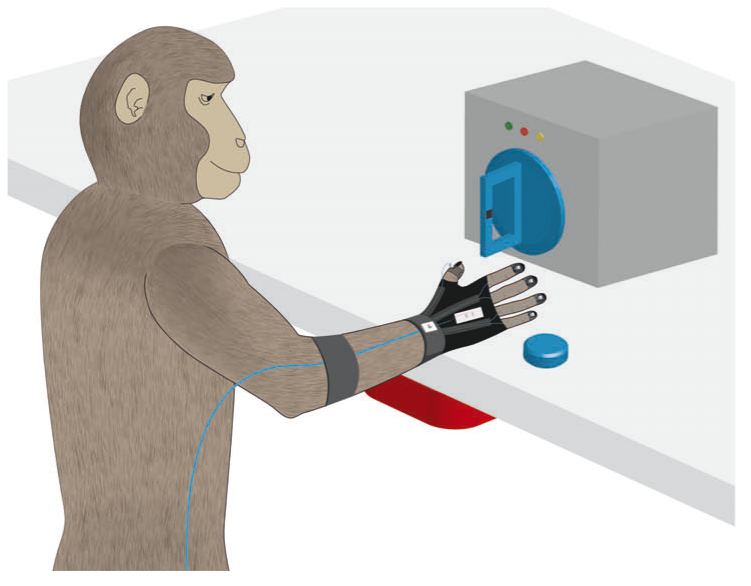

(b)

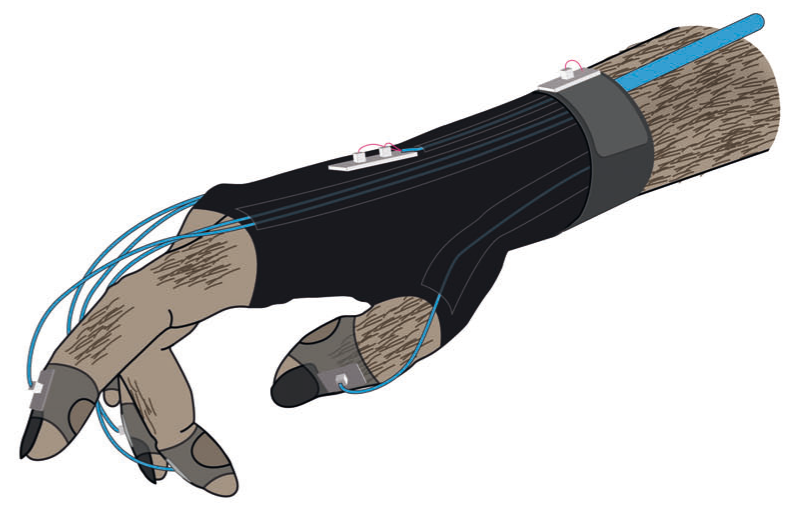

Figure 1. Behavioral setup and tracking glove. (a) Setup. A yellow or green LED instructs the monkey to perform either a precision or a power grip while hand and arm movements are tracked with the instrumented glove. Red LED: eye fixation point. A field generator (red box) placed below the table induces currents in the sensor coils that are proportional to distance and orientation with respect to the generator. (b) Tracking glove. The tracking system consists of a flexible fingerless glove holding a reference sensor on the hand's dorsum, flexible finger-caps holding a sensor on each fingertip, and micro-tubes guiding the cables and a cable strain relief from the wrist to each fingertip sensor. A wristband holds the arm sensor and fixates the glove with a Velcro (hook-andloop) fastener.

\section{Sensor System}

To track hand and arm movements, we used the electromagnetic tracking system WAVE (Northern Digital, Waterloo, CA). The technology is based on generating near-field, low frequency magnetic fields from an assembly of stationary antenna coils (transmitters), which are detected with one sensor coil (5 DOF sensor) or with two sensor coils arranged perpendicular to one another (6 DOF sensor). The field generator (figure 1(a), red box below table) induces currents in the passive receiver coils that are proportional to the strength of the current in the transmitter, the distance between transmitter and receiver, and the orientation of the receiver. Sequential activation of the transmitter coils results in a sensor output of a set of linearly independent vector fields. This output contains sufficient information to determine the position and orientation of the sensor relative to the transmitter (Raab, 1982, Bashashati et al., 2006). The carrier frequency of the system is kept proprietary, but measurements show that it operates in the lower kHz frequency band.

The 5 DOF sensors (dimensions: $3 \times 3 \times 3 \mathrm{~mm}$ ) provide information on the 3-dimensional spatial position $(x, y, z)$ as well as on the yaw and pitch angle with respect to the coordinates of the field generator; 6 DOF sensors (dimensions: $9.5 \times 4.5 \times 4 \mathrm{~mm}$ ) additionally provide a roll angle. The system 
allows sensor tracking within a defined measurement volume of $0.5 \times 0.5 \times 0.5 \mathrm{~m}$ at a sampling rate of $100 \mathrm{~Hz}$. The accuracy of the sensors for static measurements is $0.6 \mathrm{~mm}$ root mean square (RMS) for position and $0.2^{\circ} \mathrm{RMS}$ for orientation, whereas for dynamic tracking the accuracy is $1.5 \mathrm{~mm}$ RMS for position and $0.6^{\circ}$ RMS for orientation (Northern Digital Inc., 2010).

\section{Setup}

Electro-magnetic tracking systems do not depend on line-of-sight, but are influenced by the presence of conductive or ferromagnetic materials (Raab et al., 1979). For this reason, metal was avoided as much as possible within the setup, on the animal chair, and on the grasping manipulandum. Distortions caused by non-ferromagnetic materials such as Titanium or stainless steel (DIN 1.441) are negligible (Kirsch et al., 2006).

\section{Software}

To control the tracking device and for data acquisition, a graphical user interface was implemented in Matlab (The MathWorks Inc., Natick, MA). The software allowed real-time acquisition of the sensor data as well as online modeling of the hand. The computed hand was visualized at up to 25 frames per second (though recording occurred at $100 \mathrm{~Hz}$ ). Time-critical functions were implemented in $\mathrm{C}$ and compiled as Matlab MEX-files. This resulted in an iteration time below $10 \mu \mathrm{s}$. Additional options allowed the user to send data over UDP (User Data Protocol) or a serial interface. This made the system compatible with a wide range of bio-signal recording systems such as RZ2 (Tucker Davis Technologies, Alachua, FL) or Cerebus (Blackrock Microsystems, Salt Lake City, UT), which facilitated synchronous recordings of hand kinematics and multi-channel electrophysiological signals.

\section{Hand and arm model}

The computation of the monkey's hand position was based on six magnetic sensors (figure 2). A reference sensor (6 DOF) was placed on the hand's dorsum and defined the local coordinate system of the hand (S6). In this coordinate system, the positions of metacarpal-phalangeal (MCP) joints (points $A_{1}-A_{5}$ ) were fixed. Their relative position to the reference sensor (S6) was determined either by measuring their distances to the center of the sensor or with a calibration routine as described below. Each fingertip sensor (S1-S5) provided its position and orientation of the distal phalanx in spatial coordinates. From this sensor data the position of the fingertips $\left(T_{1}-T_{5}\right)$ and the distal interphalangeal (DIP) joints $\left(C_{1}-C_{5}\right)$ were calculated.

The joints of a single finger are anatomically restricted to move within a single plane. Therefore, the proximal interphalangeal (PIP) joint (point B) is located within this plane and can be calculated when 
the length of the finger phalanges (line $A B$ and $B C$ ) are known (figure 2(b)). Geometrically, two solutions can be found in the plane for the PIP joint position (defined as B and B' in figure 2(b)), but only the case where the PIP joint and the fingertip $T$ lie on opposite sides of the line $A C$ is physiological. From these joint positions, which also determine the orientation of the phalanges, all 20 finger joint angles could be extracted, including flexion, extension of all finger joints (DIP, PIP, MCP), and adduction/abduction (spread) of each finger at the MCP joint.

The modeling of the arm was realized by employing a sensor (S7) on the distal forearm close to the wrist, which measured the orientation of the lower arm. Since the elbow joint is located on the sensor's axis, its location can be calculated if the length of the forearm is known. Finally, we assumed the shoulder position of the animal to be fixed in space; this allowed us to compute a full model of the monkey's arm (i.e., 7 DOF) including the elbow angle and flexion/extension, adduction/abduction and lateral and medial rotation of the shoulder as well as flexion/extension, adduction/abduction, and pronation/supination of the wrist.

As mentioned above, the position of the MCP joints relative to the reference sensor can be measured manually. To improve this step, we developed a calibration method that determines the MCP joint positions automatically: when a finger is stretched, the sensor axis points in the direction of the MCP joint. Shifting the position of the fingertip along this axis by the length of the finger determines the position of the MCP joint. To apply this routine we placed the animal's outstretched hand with the palm down on the table in front of it. The position of the MCP joints were computed for 1 second (100 samples) and averaged. Resulting MCP positions (relative to the reference sensor) were then used during real-time tracking of the hand but could also be adjusted offline if necessary.

A detailed mathematical description of the hand and arm model and of the calibration routine is presented in the appendix. 
(a)

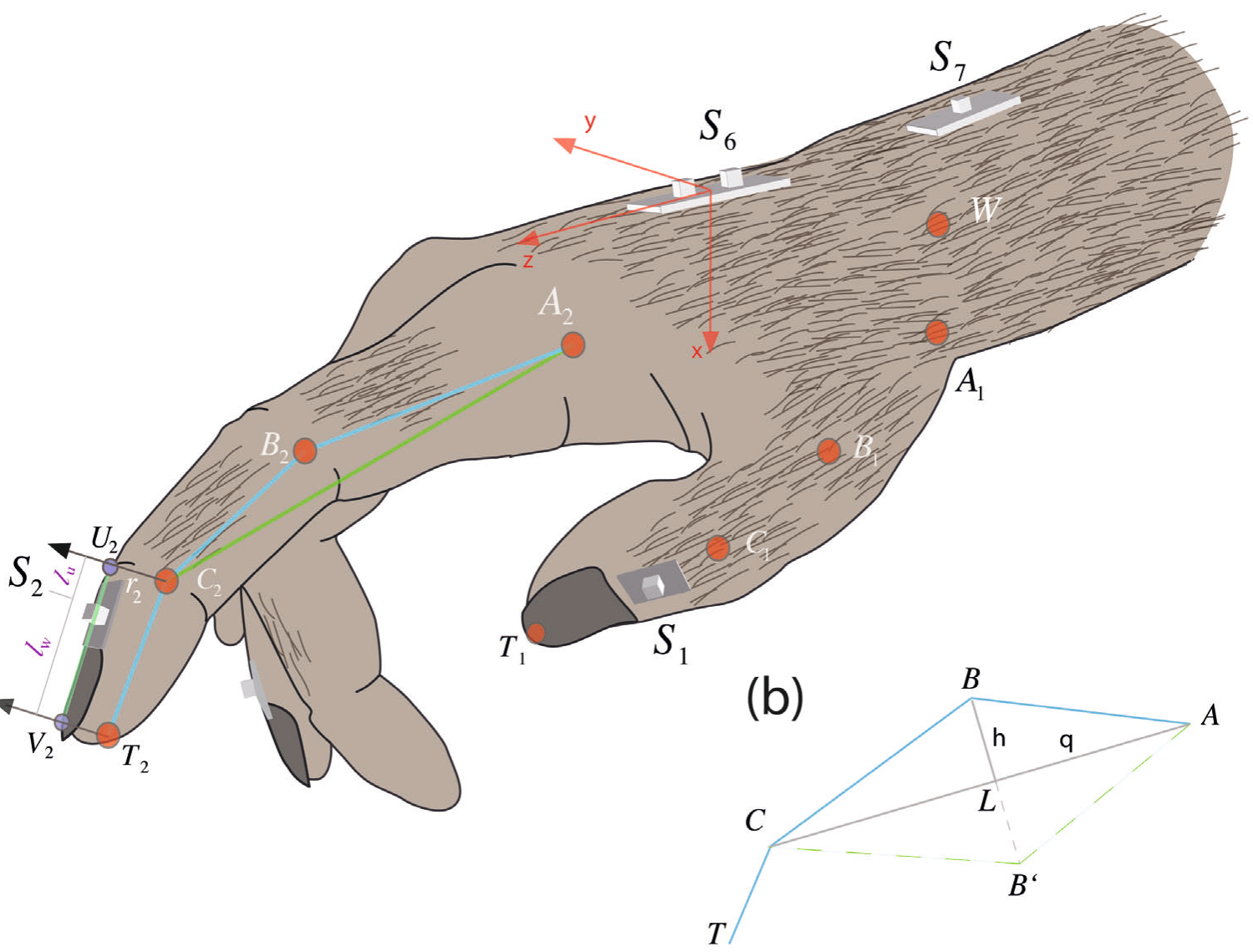

Figure 2. Hand model. (a) Seven magnetic sensors $\left(S_{1}-S_{7}\right)$ define seven points and orientations on the monkey's hand. A reference sensor $\left(S_{6}\right)$ is placed on the hand's dorsum providing the $x, y$, and z-position in $3 D$ space as well as the hand's yaw, pitch, and roll angle (6DOF). $S_{6}$ defines the origin and orientation of the local coordinate system of the hand. Sensors $S_{1}-S_{5}$ are placed on the fingertips of the hand (only $S_{1}$ and $S_{2}$ are shown), providing their position and the orientation of the distal phalanx in order to find points $U$ and $V$ on the finger's surface. The projection of $U$ and $V$ to the distal phalanx center leads to the DIP joint (C) and the fingertip $(T)$. Since $A, C$, and $T$ are moving within the same plane, the PIP joint position (B), is also located on this plane and can be computed. (b) From the measured length of the proximal and middle phalanges ( $A B$ and $B C$ ) and the calculated distance (AC) two solutions emerge for the PIP joint in the plane: $B$ and $B^{\prime}$. However, the correct solution is the one where $B$ and $T$ are on opposite sides of the line $A C$, which is the physiological case.

\section{In vitro evaluation methods}

\section{Static accuracy}

To determine the accuracy of the hand tracking system and the subsequent hand model calculations, we placed the sensor-coils on a wooden hand model of five fingers, which could each be moved in 3 DOF. After calibration, the orientation of the proximal phalanx (PPO), middle phalanx (MPO), and distal phalanx (DPO) were systematically altered within their movement-range in steps of about 5 degree and their corresponding elevation angles recorded with the magnetic sensor system. For verification, a high-resolution camera (Canon EOS 550D with lens Canon EF 70-200 mm) was placed 
parallel to the hand to take photos of the markers placed on each finger joint (MCP, PIP, and DIP) and the fingertip. Images were loaded into Matlab to detect the markers manually for computing all phalanx orientations. The camera resolution of $3456 \times 5184$ pixels allowed a theoretical resolution of $0.02^{\circ}$. Real joint angles, based on the photo images, were compared with computed angles of the joints (figure 3(a)).

\section{Dynamic tracking}

To confirm a sufficiently fast tracking speed for detecting fast reaching- and grasping-movements of rhesus monkeys, we placed the sensors again on the wooden hand and moved it through the measurement volume with various speeds. We computed the hand model while moving the wooden hand at speeds up to $3.2 \mathrm{~m} / \mathrm{s}$. The computed orientations of the phalanges during movements were compared to their static orientation to evaluate the error of movement speed on tracking accuracy (figure 3(b)).

\section{In vivo evaluation methods}

\section{Biosignal interference}

Developing a device compatible with neural recordings requires testing for possible bio-signal interferences. Bashahati et. al. (2006) showed that electro-magnetic tracking with this device does not disturb EEG recordings within a frequency range of 0.1-55 Hz. However, the potential disturbance on the extracellular recording of action potentials (frequency band: $300-7000 \mathrm{~Hz}$ ) has not yet been investigated.

To evaluate this possible interference, we recorded spiking activity from four implanted floating microelectrode arrays (FMA; MicroProbe Inc, Gaithersburg, MD, USA) in a separate animal that had been implanted previously: two arrays had been placed in the ventral premotor cortex (F5) on the bank of the arcuate sulcus (AS) and two further arrays had been implanted in the anterior intraparietal cortex (AIP) toward the lateral end of the intraparietal sulcus (ISP). A detailed description of the electrode arrays and the surgical procedures is presented in Townsend et al. (2011). Neural signals were amplified (300x) and digitized with 16-bit resolution (0.25 $\mu \mathrm{V} / \mathrm{bit})$ at 30 kS/s using a Cerebus Neural Signal Processor (Blackrock Microsystems, Salt Lake City, UT).

Neurons were recorded under 4 conditions, each lasting 2 minutes: tracking system disabled (C1), or tracking system enabled and the field-generator placed at a distance of $50 \mathrm{~cm}$ (C2), $30 \mathrm{~cm}$ (C3), or 10 $\mathrm{cm}$ (C4) from the animal's head. 
As a first test, we investigated the noise levels of the recordings for different distances (conditions C1-C4) to evaluate how much the interference affected the quality of the recordings. The noise level (3x s.d.) was determined for the continuous and filtered (bandpass $0.3-7 \mathrm{kHz}$ cut-off frequency, Butterworth $4^{\text {th }}$ order) data (figure 4(b)) over 56 channels.

In a second test, we investigated possible effects on the quality of the detected waveforms. For this, we compared the waveforms of 99 neurons for condition C1 against C2-C3. For spike sorting, we used manual and automatic spike sorting techniques in Plexon-Offline-Sorter (Plexon Inc., Dallas, TX, USA). To determine possible effects on the waveforms for conditions $\mathrm{C} 1-\mathrm{C} 3$, we first subtracted all waveforms of a unit from its mean waveform in condition C1 (device off). From this, the RMS value was calculated for each unit separately for conditions C1-C3 (figure 5). The RMS values between these conditions were compared using a paired t-test $(p<0.05)$.

\section{Repeatability}

The sensor's repeatability was evaluated by using a standardized evaluation method (Dipietro et al., 2003). To proof the variability in a recording session, the first 100 successful trials were separated into 10 consecutive blocks of 10 trials (Overduin et al., 2010). The measured joint angles were timealigned and averaged over 0.8 seconds while the monkey pressed the hand-rest button. An array $\left(X_{i j k}\right), i=1, \ldots, 10, j=1, \ldots, 10, k=1, \ldots, 27$ was finally obtained to specify the data for the $i^{\text {th }}$ trial in the $j^{\text {th }}$ data block and related to the $\mathrm{k}^{\text {th }}$ joint angle. For each session the range was defined as $R_{k}=$ $\left(\max _{j}\left(\bar{X}_{j k}\right)-\min _{j}\left(\bar{X}_{j k}\right)\right)$ where $\bar{X}_{j k}=\frac{1}{10} \sum_{i=1}^{10} X_{j k}$. In a next step we calculated the average of $R_{k}$, the standard deviation (s.d.) of the $\bar{X}_{j k}$ values, and the average of the s.d. across all joint angles. The average range and the average s.d. across the joint angles were used to define the glove's repeatability.

In an additional test we computed the repeatability in between days. For this, we defined the first 100 correct trials of each day as a distinct block and computed the average range and s.d. across these days. To estimate the day-to-day variability of the MCP positions, we calculated the distance between adjacent MCP joints: $M C P(k, n)-M C P(k+1, n)$, [k: finger number, $n$ : day number]. The standard deviation over all days was then averaged for all joints $k$, describing the variability of the MCP estimation. 


\section{Subject}

Kinematic-data was recorded from an 8-year-old female rhesus monkey (Macaca mulatta) with a weight of $7.0 \mathrm{~kg}$. In sequential steps, the monkey was trained to tolerate the sensor glove by applying positive reinforcement training techniques (Laule et al., 2003, Prescott et al., 2005). We started to condition the animal to hold hands for periods of more than one minute. In the next step we manipulated the animal's hand with pieces of fabric around its wrist and fingers until the animal tolerated the whole glove. Training duration was about 4 month until the animal tolerated the glove. This time includes extensive testing of different prototypes of the glove. It should be noted that this first animal was by its nature quite active and distractible. A second, more relaxed animal (not included in this study) could be trained to tolerate the glove in about a month.

For the experiment, we recorded hand kinematics in 5 daily sessions of about 2 hour duration.

\section{Behavioral training}

The animal was trained to perform power or precision grips in a delayed grasping task, as described in detail in previous publications (Baumann et al., 2009, Fluet et al., 2010) (figure 1(a)). In short, the animal had to place its hand on a capacitive switch to initiate a trial. Then, two LEDs indicated the grip type and the start of the grasping task. Force sensors and push buttons were used to detect power or precision grips. All correct trials were rewarded with a fixed amount of juice, and the animal could initiate the next trial after a short intertrial interval of $500 \mathrm{~ms}$. Error trials were immediately aborted without giving a reward and were followed by a longer intertrial interval (1500 $2500 \mathrm{~ms}$ ). The animal's behavior and all stimulus presentations were controlled using custom-written behavioral control software implemented in LabView Realtime (National Instruments), which allowed a time resolution of $1 \mathrm{~ms}$. 


\section{Results}

\section{In vitro testing}

After the assembly of the glove, we first performed in-vitro tests to measure the static and dynamic accuracy of the hand model. This was performed on a wooden hand (see chapter: "In vitro evaluation methods").

\section{Static accuracy}

We used the wooden hand model to evaluate the accuracy of the hand tracking system. Optical markers were placed on the wooden hand to precisely measure the finger joint angles photographically. Real joint angles were then compared with the computed joint angles from our model (see Fig. 3(a)). Across all measurements, we found an absolute error of $1.73 \pm 2.2^{\circ}$ (mean \pm s.d.) for the proximal (PPO), $1.65^{\circ} \pm 2.03^{\circ}$ for the middle (MPO), and $0.61^{\circ} \pm 0.66^{\circ}$ for the distal phalanx orientation (DPO). This confirmed that the data glove is sufficiently accurate for finger tracking in static (no motion) conditions.

\section{Dynamic tracking}

We then used the wooden hand to evaluate the affect of fast movements on measurement accuracies. For this, we brought the hand in a fixed posture and compared the joint angles measured in static position with those measured while the hand was moving with various speeds (Fig. 3(b)). We found slightly, but significantly higher measurement errors when the speed increased (Pearson's correlation coefficient: $r=0.29, p<0.01$ ). We measured an absolute error of $0.81 \pm 0.84^{\circ}$ (mean \pm s.d.) for DPO, $2.11 \pm 3.26^{\circ}$ for $\mathrm{MPO}$, and $2.41 \pm 3.36^{\circ}$ for PPO. The manufacturers accuracy specifications $\left(0.60 \pm 0.7^{\circ}\right)$ therefore could be confirmed, but only for speeds below $0.5 \mathrm{~m} / \mathrm{s}$. These errors are generally tolerable, in particular since they do not accumulate across various finger joints. In other words, position and orientation of the fingertip is measured directly by the fingertip sensor and is therefore most accurate. Overall, these in vitro results confirmed the suitability of this tracking method for hand and finger tracking in monkeys. 
(a)

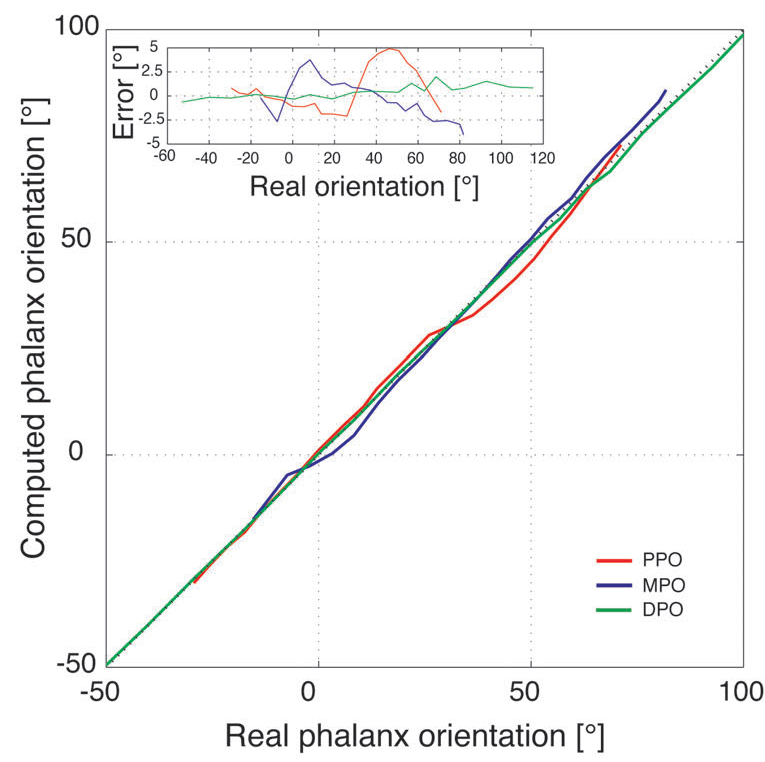

(b)

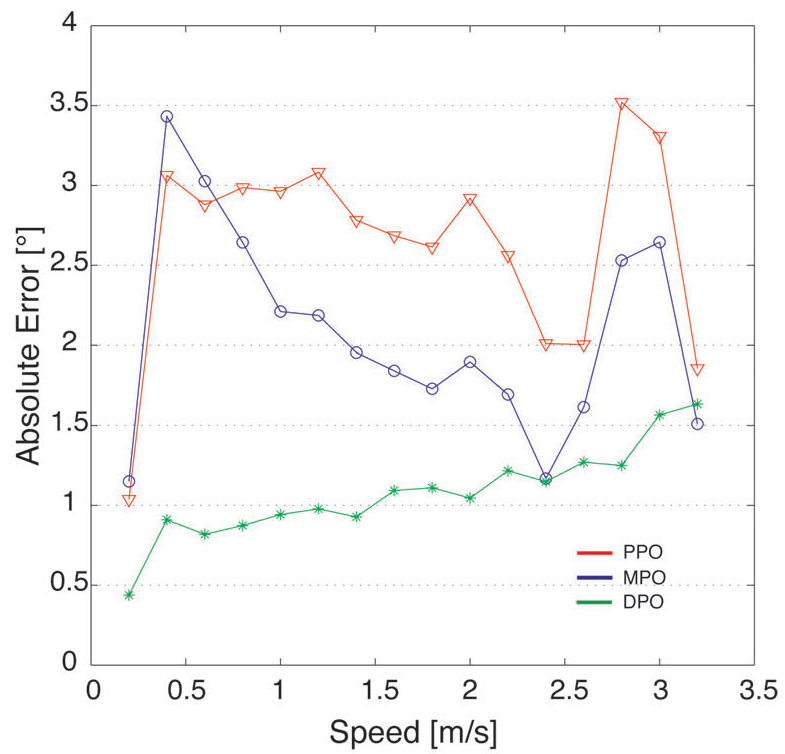

Figure 3. Tracking accuracy. A wooden hand model was used to verify the static and dynamic accuracy of the computed hand model for the proximal (PPO), middle (MPO), and distal phalanx orientation (DPO). (a) Static accuracy. The wooden hand was kept static while altering the orientation of the finger phalanges stepwise over their full range of motion. For every step, the computed phalanx orientation was plotted vs. its real orientation, as determined by visual markers and a high-resolution camera. The inlay shows the measurement error of the phalanx orientation (real - computed) over the full motion range. (b) Dynamic accuracy. For testing dynamic accuracy, the wooden hand was moved with various speeds while the orientation of all phalanges were kept constant. The measurement error was then defined as the difference between the dynamic and the static orientation of a phalanx. The panel shows the absolute mean error for the three phalanges of a finger (PPO, $M P O, D P O$ ) vs. the speed of the hand (bin size $=0.2 \mathrm{~m} / \mathrm{s}$ ).

\section{In vivo testing}

\section{Usability}

Using positive reinforcement training, we have trained a macaque monkey to wear the data glove in a primate chair and within the experimental setup. The animal performed a delayed grasping task, which it had previously learned for a different experiment. Wearing the glove did not cause a decline in task performance (with glove: $89.8 \pm 2.1 \%$ correct trials; without glove: $85 \pm 2.3 \%$ ), nor did it reduce the number of trials performed by the animal per session. Hand and arm kinematics of the macaque monkey were recorded over five consecutive days. In total about 6900 grip trials were recorded. The animal performed $1107 \pm 77$ (mean \pm s.d.) correct trials per day. By comparison, during the five training days prior to the experiments, when the animal was not wearing the glove, it performed an average of $1029 \pm 142$ (mean \pm s.d.) correct trials per day. The monkey tolerated the 
glove during recording sessions. In addition, the glove and sensors were robust enough to withstand situations in which the monkey tried to remove the glove. The specific glove design allowed attaching the glove to the monkey's hand in 5-10 minutes before each recording session.

\section{Measurement repeatability}

Measurements repeatability addresses the question how much the measured joint angles change within a recording session or between recording days while the animal performed the well-trained grasping task. To allow a comparison of our results with previous work, we used standardized evaluation methods (see chapter: "Repeatability"). Following previous investigators (Dipietro et al., 2003) we computed the average repeatability range and the s.d. metrics across all joint angles both within a day and between different days. Repeatability within a recording session showed an average range of $5.4^{\circ}$ and an average s.d. of $1.65^{\circ}$ across all computed joint angles. In contrast, between-day repeatability had an average range of $12.7^{\circ}$ and an average s.d. of $5.6^{\circ}$.

Furthermore, we computed the distance between adjacent MCP joints and their variation across days to estimate the day-to-day variability of the computed MCP joint positions (see Methods), and found, across recording days, an average standard deviation of these distances of $1.3 \mathrm{~mm}$. Together, these results clearly demonstrate a tight and reliable fit of the glove and the sensors to the hand, which is a prerequisite for accurate tracking of hand and finger movements.

\section{Bio-signal interference}

We next tested for a possible influence of the magnetic field generator with electrophysiological recordings. Toward this end, we compared the noise level of electrophysiological recordings and the stability of single unit waveforms for different testing conditions (head-to-field-generator distances): tracking system disabled ( $\mathrm{C} 1$ ) and tracking system enabled with the field-generator at a distance of $50 \mathrm{~cm}$ (C2), $30 \mathrm{~cm}$ (C3), or $10 \mathrm{~cm}$ (C4) from the animal's head. We found a massive signal disturbance for condition C4 that effectively precluded a clear classification of spikes, but for conditions C1-C3 waveforms could be identified and sorted positively for all 99 neurons tested (see figure 5).

Continuous, band-pass filtered recordings (Fig. 4 (b)), showed a noise level (3x s.d.) of $126.8 \pm 48.9$ $\mu \mathrm{V}$ (mean \pm s.d.) for condition C4 (distance: $10 \mathrm{~cm}$ ), $44.0 \pm 10.5 \mu \mathrm{V}$ (mean \pm s.d.) for C3 $(30 \mathrm{~cm}$ ), 41.9 $\pm 9.9 \mu \mathrm{V}$ (mean \pm s.d.) for $\mathrm{C} 2(50 \mathrm{~cm}$ ), and $41.6 \pm 9.4 \mu \mathrm{V}$ (mean \pm s.d.) for condition C1 (generator off). C1-C4 showed significantly different noise levels (1-way ANOVA $p<0.01$ ), which could be entirely explained by the increase of $\mathrm{C} 4$ (paired t-tests: $\mathrm{C} 1-\mathrm{C4}, \mathrm{C2}-\mathrm{C4}, \mathrm{C3}-\mathrm{C4}$, each $\mathrm{p}<10^{-6}$ ). Importantly, the noise levels in condition C1 - C3 were not significantly different (1-way ANOVA for C1-C3, p=0.4), 
which demonstrated that a field generator distance of at least $30 \mathrm{~cm}$ did not strongly affect the electrophysiological noise levels. The amplitude spectrum of the continuous signals pinpoints the interferences of the magnetic field generator to the lower $\mathrm{kHz}$ frequency band (figure 4(c)).

Consistent with these electrophysiological continuous signal measurements, spike classification was precluded in condition C4, but could be easily done in conditions C1-C3. Spike waveform variability measured in RMS showed a non-significant RMS increase of $0.2 \mu \mathrm{V}$ between condition $\mathrm{C} 1$ and $\mathrm{C} 2$ (paired t-test, $p>0.05)$ and a significant increase of $1.18 \mu \mathrm{V}$ between $\mathrm{C} 1$ and $\mathrm{C} 3(\mathrm{p}<0.05)$. A comparison of RMS values of $C 2$ and $C 3$ vs. $C 1$ is illustrated in Fig. 5. Although the noise level increased slightly, but significant, a clear classification of all recorded units was still possible and the waveforms remained unchanged (figure 5 , inset). These results demonstrate that single unit recordings are possible and distortions of waveforms are minimal if the distance of the field generator from the animal's head is at least $30 \mathrm{~cm}$, which could easily be accommodated.

(a)

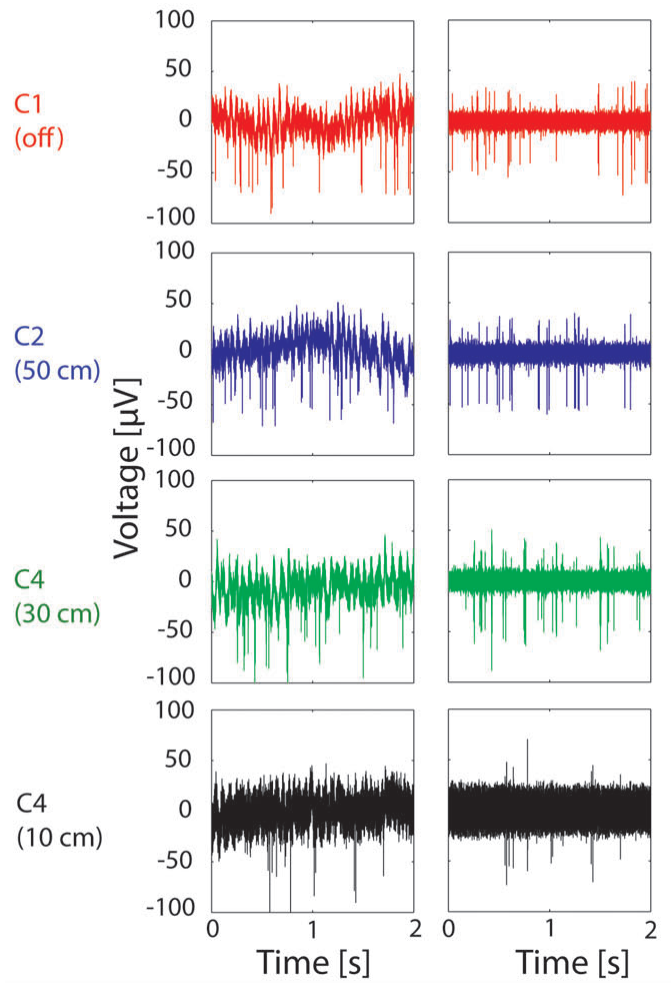

(c)

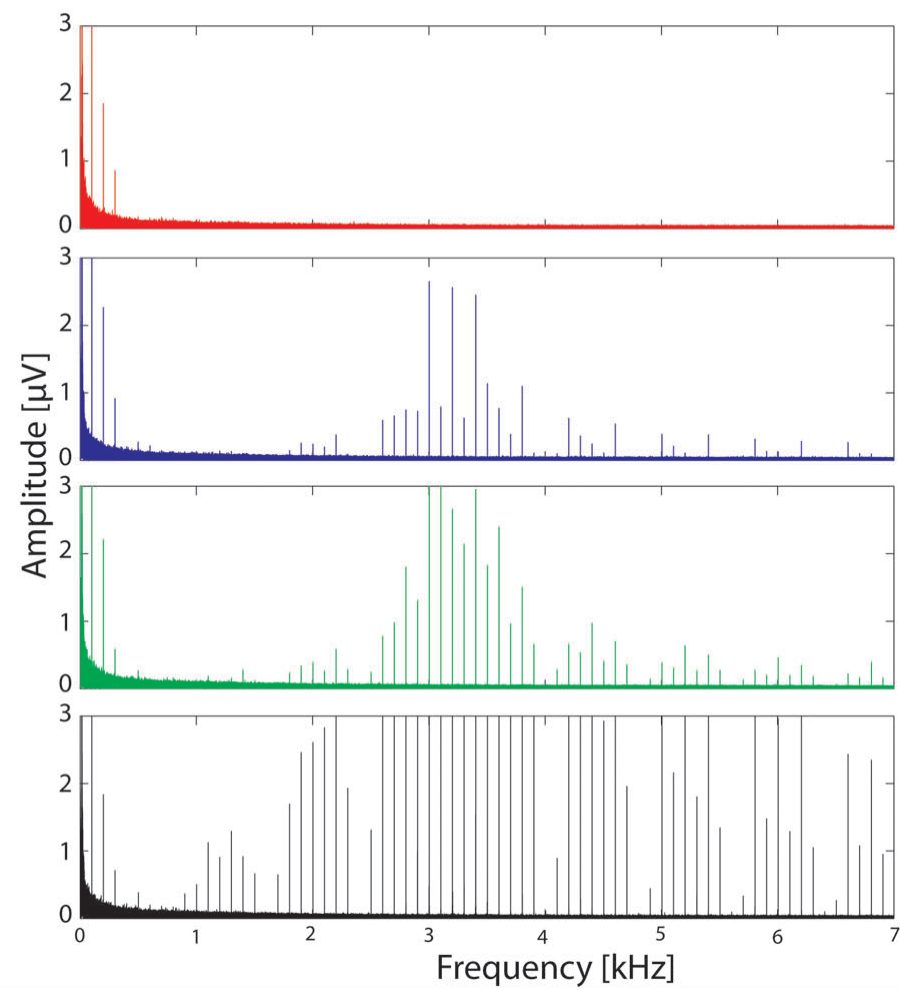

Figure 4. Electrophysiological signal disturbance by the field generator. The effect on extracellular recordings was tested with the device off (C1, red), and while operating at a distance of $50 \mathrm{~cm}(\mathrm{C} 2, \mathrm{blue}), 30 \mathrm{~cm}$ (C3, green) or $10 \mathrm{~cm}$ (C4, black) to the head of the monkey. (a) Raw, continuous extracellular recordings of an example channel. (b) Same data, bandpass filtered (0.3-7 kHz; $4^{\text {th }}$ order Butterworth). (c) Amplitude spectrum of the raw extracellular recording. 


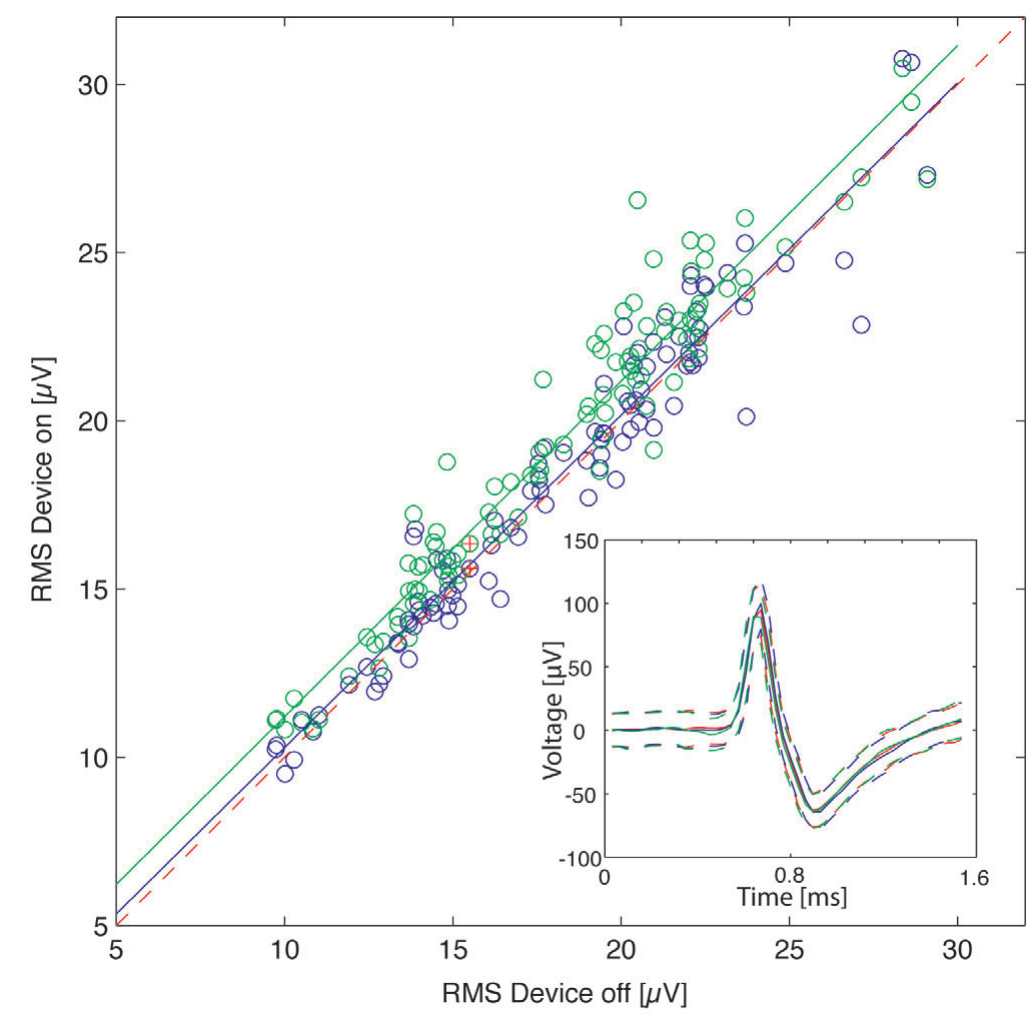

Figure 5. Stability of spike waveforms. 99 neurons were detected on 56 channels under three conditions: (C1) field generator off, (C2) head-generator-distance $=50 \mathrm{~cm}$, and (C3) head-generator-distance $=30 \mathrm{~cm}$. For each condition C1-C3, the waveforms of a unit were subtracted from the mean waveform in C1 and the residual RMS value calculated over all samples per condition. The RMS value of condition C2 (blue circles) and condition C3 (green circles) is plotted vs. condition C1 separately for each neuron. Dashed red line: unity line; blue solid line: least square fit of RMS in C2 vs. C1; green solid line: same for C3 vs. C1. Inlay: spike shapes of an example unit in condition C1 (red), C2 (blue), and C3 (green); solid line: mean spike shape, dashed lines: RMS border of the waveforms.

\section{Hand grasping kinematics}

The sensor's position and orientation were acquired with a sampling rate of $100 \mathrm{~Hz}$. The model of the hand was updated in real-time at this rate (figure $6(b-c))$. Based on the 18 joint positions, the joint angles (27 DOF) were extracted and plotted in figure 6(a). The computational model of the hand and arm includes, for each finger, the metacarpal-phalangeal (MCP) joints (flexion, extension, adduction and abduction), the proximal interphalangeal (PIP) joints (flexion, extension), and the distal interphalangeal (DIP) joints (flexion, extension), and for the wrist and arm the radiocarpal joint (flexion, extension, adduction, abduction, pronation and supination), the elbow joint (flexion, extension), and the shoulder (flexion, extension, adduction, abduction, lateral-, and medial rotation). As we will demonstrate, the full hand and arm motions could be tracked reliably across 5 daily sessions. 
The kinematics of the hand showed significant variation for precision- and power-grips (figure 6(a)). Both grip types made it necessary to rotate the wrist about $90^{\circ}$ to grasp a vertically oriented handle (see figure 1(a)). During precision-grips, this rotation was almost completely accomplished by a rotation of the shoulder, whereas during power-grips the rotation was mainly achieved by a supination of the wrist. The medial rotation and adduction of the shoulder during precision-grip were compensated by a strong extension and slight abduction of the wrist. This placed the hand in an optimal position with respect to the handle to perform the precision grip. By comparison, during the power-grip the wrist flexed to allow the fingers to encompass the handle. Other components of the reaching movement, mainly involving the shoulder (extension, adduction) and elbow (extension), were similar for both grip types, mainly because of the identical target location.

Finger movements were also markedly different between the two grip types. The aperture, defined as the distance between the tips of thumb and index finger, illustrated this variation. During power grip trials (figure $6(a)$; time $t_{1}$ ), grip aperture first increased and then decreased as the handle was grasped. In precision grip trials, by comparison, grip aperture decreased immediately (figure 6(a); time $t_{2}$ ). For power grip, all digits flexed and extended together, whereas during precision grip digits 3-5 flexed more than the thumb and the index finger. This behavior ensured enough space for thumb and index finger to perform a precise grip on the handle. Furthermore, fingers touching the handle showed a slight extension of their DIP joints (e.g. thumb during precision grip and middle and ring finger during power grip). This was a likely consequence of the pressure exerted by the fingertips on the handle. 
(a)
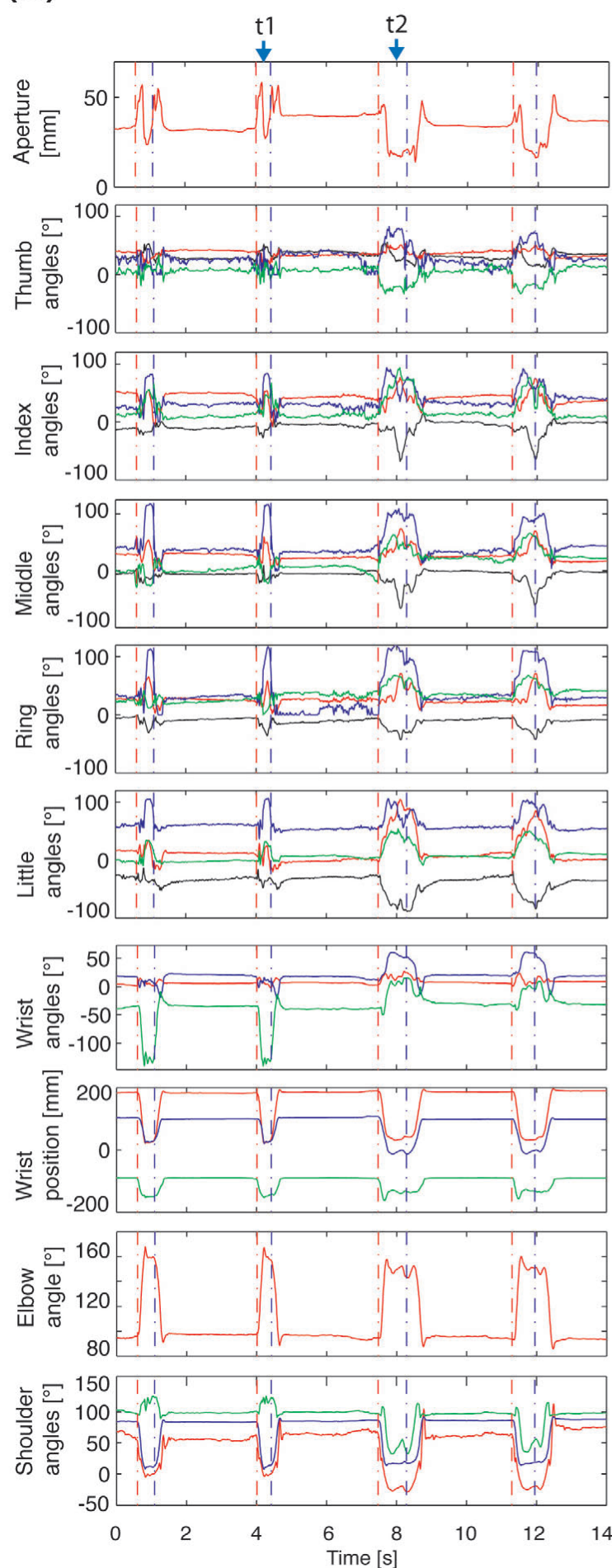

(b) Power grip (t1)

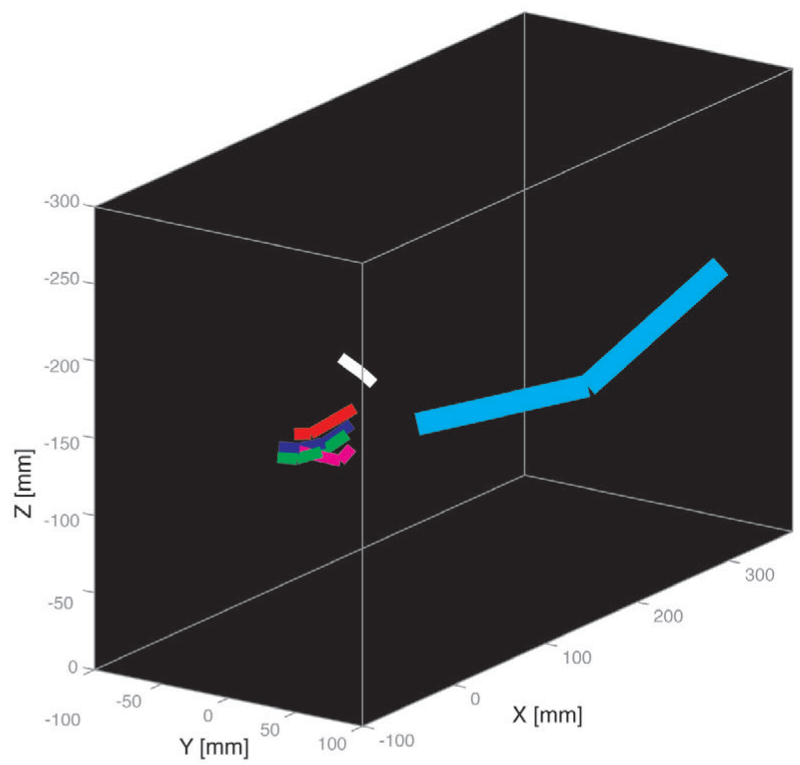

(c) Precision grip (t2)

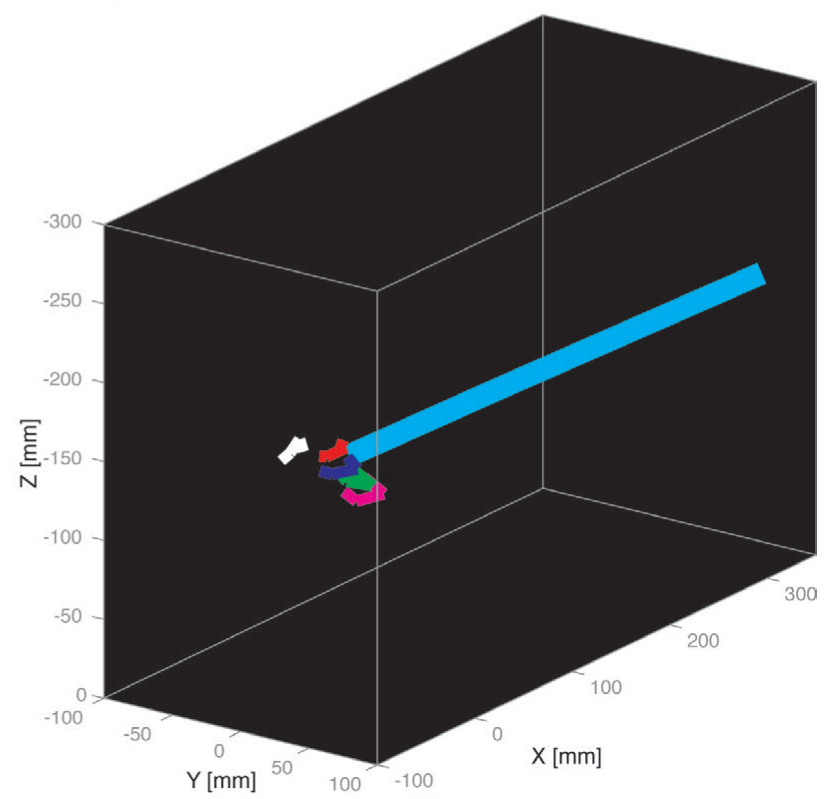

Figure 6. Hand and arm kinematics. (a) Kinematic features of hand and arm are plotted over time. Traces show the performance of two power grips and two precision grips in sequence. Subpanels from top to bottom: hand aperture (red), joint angles of thumb, index, middle, ring, and little finger (MCP in red, PIP in blue, DIP in green, spread in black), wrist orientation (adduction/abduction in red, flexion/extension in blue, pronation/supination in green), wrist position ( $x$ in red, $y$ in blue, $z$ in green), elbow angle (red), and shoulder orientation (adduction/abduction in red, flexion/extension in blue, lateral/medial rotation in green). Vertical dashed lines indicate the "go-signal" (red) and the time of reward (blue) for each trial, respectively. Screenshot of the real-time computed hand model for precision (b) and power-grip (c) at times $t 1$ and $t 2$, respectively. 


\section{Discussion}

\section{Strengths}

In this work we have demonstrated a novel method for tracking finger, hand, and arm movements of macaque monkeys based on an instrumented glove with only 7 sensors. This technique allows tracking of 27 DOF of finger, wrist, elbow, and shoulder joint angles of 18 joints and includes their position in 3-dimensional space. Our new computational hand model allows reducing the number of tracking sensors by exploiting the anatomical geometry of the primate's hand. To our knowledge, this ratio between DOF and number of sensors is higher than for any other published or commercially available method.

The reduced number of sensors and their miniaturization make the glove design well suited for kinematic hand tracking in small primates. As we have demonstrated, our computational model did not require additional sensors on the upper arm or elbow, nor at intermediate or proximal phalanges of the fingers. This made it possible to separate the glove into parts, which made the fitting of the glove on the animal's hand easy and quick. Furthermore, in full fabric gloves, actions such as wrist or finger flexion often cause the glove to slip over the skin, which would then compromise measurement accuracy (Simone and Kamper, 2005). Subdividing the glove into independently moving parts reduces this limitation. In addition, full fabric gloves interfere with tactile sensing. In our design, most parts of the arm and hand are free of fabric to reduce impairment of tactile sensation.

Our behavioral task included precision grips in the dark, and was therefore highly dependent on tactile information. Good performance in this task therefore clearly demonstrated the suitability of this glove even for complex grasping tasks. Initially, we built a full fabric glove made out of highly elastic Lycra material as a first prototype. Because of the mentioned disadvantages and the long time needed for placement (up to 15 minutes), our subsequent experience with the fingerless-glove was much superior.

Recording of 27 DOF with only 7 sensors was also possible because of the employed sensor technology, in which a single sensor coil provided 5 DOF. In comparison, single optical markers can measure only the spatial position ( 3 DOF). Furthermore, magnetic sensor coils can be tracked without dependence on line-of-sight. This makes it possible to track hand movements that are not visible for a set of cameras (e.g. grasping of hidden objects or of objects in cavities). In contrast, 3D optical tracking systems depend on line-of-sight to at least two cameras, however, more typically up to 12 cameras are used (e.g., see Vargas-Irvine et al., 2010 or Zhuang et al., 2010). This is a strong limitation for many experimental designs. Other technologies that do not depend on line-of-sight are 
electro-resistive or optical flex sensors. However, these technologies have other serious disadvantages, including non-linearity, the absence of a 3-dimensional sensor position, and their low accuracy compared to sensor coils (Simone and Kamper, 2005).

In our tests, we confirmed the manufacturer's specification for static sensor orientation accuracy of $0.6^{\circ}$ RMS. The same accuracy was also found for many joint angle computations in our model, including the most important parameters for grasping such as flexion/extension of the distal phalanx and the wrist, as well as finger and wrist adduction/abduction. Other parameters for grasp representation, such as MCP and PIP, were measured with lower precision (mean absolute errors $<2^{\circ}$ ) due to the more indirect means of computation; however these values are still better than reported values of other methods (Overduin et al., 2010). Sensor placement on the distal phalanx, as in our method, can therefore generate highly accurate measurements of fingertip position and orientation, which is particularly advantageous for the study of grip types and hand manipulation.

However, as a precaution we want to note that in our computational model, as in most others, the palm of the hand was assumed to be stiff (i.e., no palmar flexion). For this reason, the angle errors of the proximal finger angles (MCP, PIP) are expected to be slightly higher for in-vivo recordings, while in particular the fingertip position and orientation will be unaffected by this assumption due to the proximate sensor location.

Beside the accuracy of the instrumented glove, its robustness is also highly relevant. To guarantee a technology that is reliable and robust enough for applications in macaques, we took several precautions against possible damage. First, we casted the sensors and their cable connections with epoxy resin. Epoxy dries within minutes, protects the sensors, and prevents cable movements close to the soldered joints. Second, we used silicon micro-tubes to protect the sensor cables. Furthermore, we passed nylon cords through the micro-tubes parallel to the sensor cables and connected them to the fabric of the glove and the plate carrying the sensors. This provided strain relief for the cables. In our experience, such protection has proved to be sufficient, even though the animal had tried several times to remove the glove before getting used to wearing it.

\section{Limitations}

Repeatability measurements within a single recording session showed lower variations (range: $5.4^{\circ}$, s.d. $1.65^{\circ}$ ) than for human gloves (range: $7.47^{\circ}$, s.d. $2.44^{\circ}$ )(Dipietro et al., 2003) and small primate gloves (range: $7.0^{\circ}$, s.d. $\left.2.3^{\circ}\right)($ Overduin et al., 2010). The low within-day variability demonstrates a tight fit of the glove and sensors with no or minimal sensor movements. However, the between-day

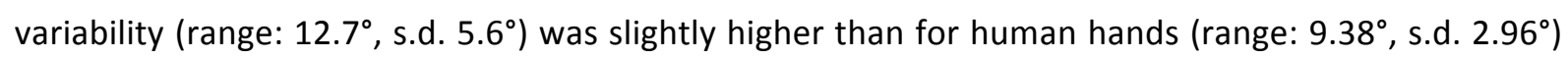
(Dipietro et al., 2003). This can be explained with the smaller dimensions of the monkey hand, where day-to-day changes of the reference sensor position have a larger effect on the computed angles. To 
our knowledge, no comparable between-day repeatability measurements have been reported for small primates so far. As a future development, the calibration method could be improved to decrease the between-day error. Instead of determining the MCP joints with the hand is stretched out on a table, calibration could be made while the animal grasps a negative mold of its own hand (Dipietro et al., 2003), which will likely decrease the variability of the obtained MCP joint positions. In general, the computational model presented here strongly depends on accurate measurements of limb segments, accurate placement of the sensors, and on the digits being represented as hinges centered in the joint space. Using elastic silicon tubes in combination with the sleeving clamp allowed highly accurate positioning of the sensors $(<1 \mathrm{~mm})$. Placing the sensors on thin plastic plates additionally prevented a possible sensor tilt. The silicon tubes ensure a close fit of the sensors to the fingers surface, to get the orientation of the distal phalanges highly accurately. Furthermore, a precise measurement of limb segments is possible, using a plaster cast model of the hand or with MR-imaging. The latter method would additionally allow finding the precise center of rotation of the finger joints, which in primates often lie within the more proximal phalangeal condyle. Furthermore, existing computational methods could be applied for reducing errors caused by sensor noise, inaccurate sensor placement, or missing data samples (Todorov, 2007).

One of the most relevant disadvantages of an electromagnetic tracking system are distortions of the electromagnetic field by ferromagnetic materials. This makes it necessary to avoid ferromagnetic materials within the setup (e.g. animal chair). However, a stable construction of the experimental setup is still possible with this constraint by using plastics of high density and mechanical strength, such as polyoxymethylen copolymer (POM-C, tensile modulus=2700 MPa), polyetheretherketon (PEEK, tensile modulus $=3600 \mathrm{MPa}$ ) or glass fiber compounds (e.g. PA 6 GF30, tensile modulus=9000 $\mathrm{MPa}$ ). Metals such as titanium or stainless steel show negligible field distortions and can also be used without constraints (Kirsch et al., 2006).

Furthermore, the magnetic field generator of the tracking system could potentially interfere with electrophysiological recordings. The field generator used for this study operated in the lower $\mathrm{kHz}$ frequency band and showed clear superpositions in the extracellular recordings. Increasing electrode-generator distance improved the signal quality significantly due to a reduction of the electromagnetic field density, which is proportional to distance-squared. Distances $>30 \mathrm{~cm}$ allowed spike sorting without limitation. Fortunately, this distance can easily be accommodated during hand and finger tracking. Furthermore, the amplitude spectrum revealed no interference below $1 \mathrm{kHz}$, which will allow the analysis of local field potentials (LFP). For further noise reduction, notch filters could be applied to suppress the field generator's carrier frequency.

The defined measurement volume produced by the field generator was limited to $0.5 \times 0.5 \times 0.5 \mathrm{~m}$. This is relatively small in comparison to optical systems that allow tracking in a measurement volume 
of several cubic-meters while providing the same or better spatial resolution. The system's measurement volume was large enough for our purpose, but could limit the design of other tasks (e.g. reaching experiments). However, the computational model of the arm extends the effective volume. As shown, the angles of elbow and arm can be computed, even if these body parts are outside of the system's measurement volume. If necessary, the measurement volume could be increased by combining the electro-magnetic system with other, e.g., optical tracking systems.

Another limitation of electro-magnetic tracking is the relatively low sampling rate, typically around $100 \mathrm{~Hz}$. This is below the frame rates provided by optical systems (up to $1 \mathrm{kHz}$ ). However, even when larger frame rates are recorded, a sampling rate of $\sim 20 \mathrm{~Hz}$ is sufficient to analyze primate hand movements in many cases (Zhuang et al., 2010).

\section{Possible applications}

The presented hand-tracking device allows recording of complete hand, finger, and arm movements together with electrophysiological signals. This enables a wide range of possible applications for this device. One of the most obvious applications is the investigation of cortical motor areas. Neuronal activity could be correlated with hand and arm kinematics in order to classify them and to understand the neuronal code. Furthermore, the static grip of an object could be compared to the motor plan activity recorded in premotor areas during delayed grasping (Baumann et al., 2009, Fluet et al., 2010, Townsend et al., 2011). As recently shown, instrumented gloves can also be used in the field of BMI to train decoding algorithms in order to reconstruct complete reach and grasp kinematics from neural activity (Vargas-Irwin et al., 2010, Zhuang et al., 2010). Further applications of the glove might include the investigation of CNS disorders in non-human primates such as spinal cord injury and stroke. In comparison to other systems, the glove presented here measures the position and the orientation of the fingers without depending on line-of-sight. This advantage allows the detection of abnormal postures, such as twisted and closed hand postures often observed after neurological impairments.

\section{Conclusion}

We have presented a new and reliable technique for finger, hand, and arm tracking of primates using a minimal number of sensors. The introduced method is convenient for monitoring reaching and grasping movements online and it is compatible with electrophysiological recordings. The system shares the main advantages of optical tracking, such as the detection of absolute positions in space, 
but does not depend on line-of-sight and could therefore be more straightforward than other systems for many neurophysiological applications.

\section{Appendix}

\section{Hand model}

Real-time computation of the hand and arm model was based on 7 sensors $\left(S_{1}-S_{7}\right)$ placed on the monkey's hand and arm (figure 2). Knowing the position and orientation of the sensors makes it possible to determine, for every finger (1-5), the location of the metacarpal-phalangeal (MCP) joint (points $\left.A_{1}-A_{5}\right)$, the proximal-interphalangeal (PIP) joint $\left(B_{1}-B_{5}\right)$, the distal-interphalangeal (DIP) joint $\left(C_{1}-C_{5}\right)$, and the fingertip positions $\left(T_{1}-T_{5}\right)$. Figure 2(a) shows these labels for the thumb $\left(A_{1}, B_{1}, C_{1}, T_{1}\right)$ and the index finger $\left(A_{2}, B_{2}, C_{2}, T_{2}\right)$.

Every sensor $\mathrm{S} \in\left\{S_{1}, \ldots, S_{7}\right\}$ provides its position in Cartesian coordinates $\boldsymbol{S}^{(G)}=\left[x^{(G)}, y^{(G)}, z^{(G)}\right]^{T}$ and its orientation in quaternions $\boldsymbol{q}^{(G)}=\left[q_{0}, q_{1}, q_{2}, q_{3}\right]^{T}$ with respect to the global (field generator) coordinate system $C_{G}$. In addition, each sensor has its own coordinate system $C_{S} . \ln C_{S}$, a point $\mathrm{p}$ is defined by the position vector $\left[x^{(S)}, y^{(S)}, z^{(S)}\right]^{T}$, or in homogeneous coordinates: $\boldsymbol{p}^{(S)}=\left[x^{(S)}, y^{(S)}, z^{(S)}, 1\right]^{T}$. This point can be transformed to the global coordinate system $C_{G}$ by the matrix equation

$$
\boldsymbol{p}^{(G)}=M_{S G}^{-1} \boldsymbol{p}^{(S)}
$$

where $\mathrm{M}_{\mathrm{SG}}$ is the transformation matrix

$$
M_{S G}=\left(\begin{array}{cccc}
q_{0}^{2}+q_{1}^{2}-q_{2}^{2}-q_{3}^{2} & 2\left(q_{1} q_{2}-q_{0} q_{3}\right) & 2\left(q_{0} q_{2}+q_{1} q_{3}\right) & x \\
2\left(q_{1} q_{2}+q_{0} q_{3}\right) & q_{0}^{2}-q_{1}^{2}+q_{2}^{2}-q_{3}^{2} & 2\left(q_{2} q_{3}-q_{0} q_{1}\right) & y \\
2\left(q_{1} q_{3}-q_{0} q_{2}\right) & 2\left(q_{0} q_{1}+q_{2} q_{3}\right) & q_{0}^{2}-q_{1}^{2}-q_{2}^{2}+q_{3}^{2} & z \\
0 & 0 & 0 & 1
\end{array}\right)
$$

and $\boldsymbol{s}=[x, y, z]^{T}, \boldsymbol{q}=\left[q_{0}, q_{1}, q_{2}, q_{3}\right]^{T}$ are the sensor position and orientation in global coordinates $C_{G}$.

Specifically, a 6DOF sensor $\left(S_{6}\right)$ defines the local coordinate system $C_{L}:=C_{S_{6}}$ on the hand's dorsum, to which the matrix transformation $\mathrm{M}_{\mathrm{LG}}$ is associated that transforms points from global $\left(C_{G}\right)$ to local coordinates $\left(C_{L}\right)$. For every finger, the position A of the MCP joint is assumed to be constant in $C_{L}$ and can be measured directly or determined by a calibration procedure (see below).

To determine the remaining points $\mathrm{B}, \mathrm{C}$, and T for each finger, we first defined the points $U^{(S)}=$ $\left[0,0,-l_{U}, 1\right]^{T}$ and $V^{(S)}=\left[0,0, l_{V}, 1\right]^{T}$ in sensor coordinates, where $l_{U}$ and $l_{V}$ denote the distance of the sensor $S$ along the sensor axis to the DIP joint and the fingertip, respectively, see figure 2(a). 
These points are then transformed to global coordinates $U^{(G)}=M_{S G}^{-1} U^{(S)}, V^{(G)}=M_{S G}^{-1} V^{(S)}$ and from there to the local coordinates of the hand $\left(C_{L}\right)$ :

$$
U^{(L)}=M_{L G} U^{(G)}, V^{(L)}=M_{L G} V^{(G)} .
$$

Thereby, $M_{L G}$ denotes the transformation matrix from global $\mathrm{C}_{\mathrm{G}}$ to local coordinates $\mathrm{C}_{\mathrm{L}}$. To determine the position of the DIP joint $C$ and of the fingertip $T$, points $U$ and $V$ are shifted orthogonally to the sensor axis to the finger's center. To compute this, we note that points $A, U$, and $V$ are anatomically restricted to move within a plane (see figure 2). A unit vector normal to this plane is $e(\overrightarrow{A U} \times \overrightarrow{U V})$, where $\boldsymbol{e}(\boldsymbol{x}):=\boldsymbol{x} /\|\boldsymbol{x}\|$ denotes the unit vector pointing in the direction of vector $\boldsymbol{x}$. Then $\boldsymbol{n}=$ $\boldsymbol{e}((\overrightarrow{A U} \times \overrightarrow{U V}) \times \overrightarrow{U V})$ lies within this plane, is orthogonal to $U V$, and has length 1 . From this and the distal radius $r$ of the finger, $C$ and $T$ can be calculated as

$$
\begin{aligned}
& C=U+r \boldsymbol{n}, \\
& T=V+r \boldsymbol{n} .
\end{aligned}
$$

Finally, to determine point $B$, we consider the triangle $(A B C)$. Points $A$ and $C$ as well as the lengths of the proximal phalanx $(A B)$ and middle phalanx $(B C)$ are known (figure $2(b))$. Using the law of cosines, the angle $\alpha$ between $(\mathrm{AB})$ and (AC) follows as $\alpha=\arccos \left(\frac{(A B)^{2}+(A C)^{2}-(B C)^{2}}{2|A B||A C|}\right)$. From this, the orthogonal projection of $B$ on the line $A C$, which we call $L$, can be computed as

$$
L=A+q \boldsymbol{e}(\overrightarrow{A C}),
$$

where $q=\cos (\alpha)|A B|$ gives the distance of $\mathrm{A}$ to L. Furthermore, $h=\sin (\alpha)|A B|$ notes the length of the line (BL). Together with the unit vector $\boldsymbol{m}=\boldsymbol{e}(\overrightarrow{A C} \times(\overrightarrow{A C} \times \overrightarrow{C T}))$, which lies in the plane $A C T$ and normal to $A C$, we can obtain $B$ as

$$
B=L+h \boldsymbol{m} .
$$

This definition of $B$ for the PIP joint location (instead of the alternate solution $B^{\prime}=L-h \boldsymbol{m}$ ) ensures that the PIP joint and the fingertip position T are always on opposite sides of the line AC, as it is the case during naturally occurring finger movements.

\section{Arm model}

The computation of the subject's arm position is realized with the sensor $S_{6}$ on the hand dorsum and sensor $S_{7}$ on the glove wristband (see figure 2(a)). To create the arm model, the position of the wrist 
joint $W^{(L)}=\left[x^{(L)}, y^{(L)}, z^{(L)}, 1\right]^{T}$ is determined by manual measurement and transformed to global coordinates $C_{G}$ by

$$
W^{(G)}=M_{L G}^{-1} W^{(L)}
$$

Then, $W^{(G)}$ and the orientation $\boldsymbol{q}_{7}^{(G)}=\left[q_{7,0}, q_{7,1}, q_{7,2}, q_{7,3}\right]$ of the sensor $\left(\mathrm{S}_{7}\right)$ define a new coordinate system $C_{W}$ of the wrist and the associated transformation matrix $\mathrm{M}_{\mathrm{WG}}$ that transforms points from global $\left(C_{G}\right)$ to wrist coordinates $\left(C_{W}\right)$.

In $C_{W}$, the position of the wrist joint is the origin $W^{(W)}=[0,0,0,1]^{T}$ and the elbow joint position is given by $E^{(W)}=\left[0,0,-l_{e}, 1\right]^{T}$, where $l_{e}$ is the distance of the wrist joint to the elbow joint. Wrist and elbow position can be transformed to global coordinates $C_{G}$ by

$$
\begin{aligned}
W^{(G)} & =M_{W G}^{-1} W^{(W)} \\
E^{(G)} & =M_{W G}^{-1} E^{(W)} .
\end{aligned}
$$

Finally, the shoulder position $H^{(G)}$ is assumed to be fixed in space and can be measured directly. This completes the arm model consisting of the global shoulder $(H)$, elbow $(E)$, and wrist $(W)$ position.

\section{Calibration}

To avoid measuring local distances between sensor $S_{6}$ and the MCP joints (point $A$ ) for every finger, we developed an online calibration method. The monkey was trained to press its hand onto a flat surface with all fingers extended. The surface was arranged orthogonally to the field generator's zaxis that points downward. Then for each finger, we defined $K^{(S)}=\left[0,0,-\left(|A B|+|B C|+l_{U}\right), 1\right]^{T}$ as the point along the axis of the fingertip sensor $S$ just on top of $A$. This point was transformed first to global coordinates $K^{(G)}=M_{S G}^{-1} K^{(S)}$ and then shifted towards the palm (along the z-axis) by finger radius $r$, which led to the MCP joint position in global coordinates $A^{(G)}=K^{(G)}+[0,0, r, 1]^{T}$ and in local coordinates $\mathrm{C}_{\mathrm{L}}$ of the hand: $A^{(L)}=M_{L G} A^{(G)}$. 


\section{References}

Bashashati A, Noureddin B, Ward RK, Lawrence PD, Birch GE (2006) An experimental study to investigate the effects of a motion tracking electromagnetic sensor during EEG data acquisition. IEEE Transactions on Biomedical Engineering 53:559-563.

Baumann MA, Fluet MC, Scherberger H (2009) Context-specific grasp movement representation in the macaque anterior intraparietal area. The Journal of Neuroscience 29:6436-6448.

Castro MC, Cliquet A, Jr. (1997) A low-cost instrumented glove for monitoring forces during object manipulation. IEEE Transactions on Neural Systems and Rehabilitation Engineering 5:140-147.

Dipietro L, Sabatini AM, Dario P (2003) Evaluation of an instrumented glove for hand-movement acquisition. Journal of Rehabilitation Research and Development 40:179-189.

Fluet MC, Baumann MA, Scherberger H (2010) Context-specific grasp movement representation in macaque ventral premotor cortex. The Journal of Neuroscience 30:15175-15184.

Gentner R, Classen J (2009) Development and evaluation of a low-cost sensor glove for assessment of human finger movements in neurophysiological settings. Journal of Neuroscience Methods 178:138-147.

Georgopoulos AP, Schwartz AB, Kettner RE (1986) Neuronal population coding of movement direction. Science 233:1416-1419.

Kirsch SR, Schilling C, Brunner G (2006) Assessment of metallic distortions of a electromagnetic tracking system. Medical Imaging 2006: Visualization, Image-Guided Procedures, and Display 6141:J1410-J1410.

Laule GE, Bloomsmith MA, Schapiro SJ (2003) The use of positive reinforcement training techniques to enhance the care, management, and welfare of primates in the laboratory. Journal of Applied Animal Welfare Science 6:163-173.

Lemon RN (2008) Descending pathways in motor control. Annual Review of Neuroscience 31:195-218.

MacKenzie IS (1995) Input devices and interaction techniques for advanced computing. In: Virtual environments and advanced interface design (Barfield, W. and Furness, T. A., eds), pp 437-470 New York ; Oxford: Oxford University Press.

Murata A, Fadiga L, Fogassi L, Gallese V, Raos V, Rizzolatti G (1997) Object representation in the ventral premotor cortex (area F5) of the monkey. Journal of Neurophysiology 78:22262230.

Murata A, Gallese V, Luppino G, Kaseda M, Sakata H (2000) Selectivity for the shape, size, and orientation of objects for grasping in neurons of monkey parietal area AIP. Journal of Neurophysiology 83:2580-2601.

National Research Council (2003) Guidelines for the care and use of mammals in neuroscience and behavioral research. Washington, D.C.: National Academies Press.

Northern Digital Inc. (2010) The Wave Tracking System. Company Brochure.

Overduin SA, Zaheer F, Bizzi E, d'Avella A (2010) An instrumented glove for small primates. Journal of Neuroscience Methods 187:100-104.

Prescott MJ, Bowell VA, Buchanan-Smith HM (2005) Training laboratory-housed non-human Primates, part 2: resources for developing and implementing training programs. Animal Welfare 4:133-148.

Raab FH (1982) US Patent No. 4346348

Raab FH, Blood EB, Steiner TO, Jones HR (1979) Magnetic Position and Orientation Tracking System. IEEE Transactions on Aerospace and Electronic Systems 15:709-718.

Schieber MH (1991) Individuated finger movements of rhesus monkeys: a means of quantifying the independence of the digits. Journal of neurophysiology 65:1381-1391.

Schieber MH, Hibbard LS (1993) How somatotopic is the motor cortex hand area? Science 261:489-492.

Simone LK, Kamper DG (2005) Design considerations for a wearable monitor to measure finger posture. Journal of Neurengineering and Rehabilitation 2:5. 
Simone LK, Sundarrajan N, Luo X, Jia Y, Kamper DG (2007) A low cost instrumented glove for extended monitoring and functional hand assessment. Journal of Neuroscience Methods 160:335-348.

Todorov E (2007) Probabilistic inference of multijoint movements, skeletal parameters and marker attachments from diverse motion capture data. IEEE transactions on biomedical engineering 54:1927-1939.

Townsend BR, Subasi E, Scherberger H (2011) Grasp movement decoding from premotor and parietal cortex. The Journal of Neuroscience 31:14386-14398.

Vargas-Irwin CE, Shakhnarovich G, Yadollahpour P, Mislow JM, Black MJ, Donoghue JP (2010) Decoding complete reach and grasp actions from local primary motor cortex populations. The Journal of Neuroscience 30:9659-9669.

Williams NW (1997) The virtual hand. The Pulvertaft Prize Essay for 1996. The Journal of Hand Surgery 22:560-567.

Yun MH, Cannon D, Freivalds A, Thomas G (1997) An instrumented glove for grasp specification in virtual-reality-based point-and-direct telerobotics. IEEE Transactions on Systems, Man, and Cybernetics, Part B: Cybernetics 27:835-846.

Zhuang J, Truccolo W, Vargas-Irwin C, Donoghue JP (2010) Decoding 3-D reach and grasp kinematics from high-frequency local field potentials in primate primary motor cortex. IEEE Transactions on Biomedical Engineering 57:1774-1784.

Zimmermann TG (1985) US Patent No. 4542291

Zimmermann TG, Lanier JZ (1991) US Patent No. 4988981 




\subsubsection{Modelling of hand and arm position and orientation}

H Scherberger ${ }^{1,2}$ and S Schaffelhofer ${ }^{1}$

$\dagger$ Equal first authors

${ }^{1}$ Deutsches Primatenzentrum GmbH, 37077 Göttingen, Germany

${ }^{2}$ Department of Biology, University of Göttingen, D-37077 Göttingen, Germany 


\section{(19) United States \\ (12) Patent Application Publication Scherberger et al.}

(10) Pub. No.: US 2013/0158946 A1

(43) Pub. Date: Jun. 20, 2013

(54) MODELLING OF HAND AND ARM POSITION AND ORIENTATION

(76) Inventors: I I ansjörg Scherherger, (jottingen (I)Ii); Stefan Schaffelhofer, (jottingen (1)]i)

(21) Appl. No.:

$13 / 816,581$

(22) PCT Filed:

Aug. 10, 2011

(86) PCT No:

PCT/EP11/63771

$\$ 371(\mathrm{c})(1)$,

(2), (4) Date: Feb. 12, 2013

\section{Related U.S. Application Data}

(60) Provisional application No. 61/373,432, filed on Aug. 13, 2010.

Foreign Application Priority Data

Aug. 13, 2010

(I)] i) 10172813.7

\section{Publication Classification}

(51) Int. Cl.

G01B $7 / 00$

G06F 17/00

$(2006.01)$

(2006.01)
(52) U.S. CI.

CPC . G01B 7/00 (2013.01); G06F 17/00 (2013.01)

USPC

$702 / 151$

\section{ABSTRACT}

The present invention provides a method for modelling a position and orientation of a hand with as small a number of sensors as possible. A first sensor is attached on a phalanx. distalis of a finger. The first sensor is adapted to provide information on at least five degrees of freedom that correspond to three translations, yaw and pitch. $\Lambda$ second sensor is placed at a fixed position relative to a dorsum or palm of the hand. The second sensor is adapted to provide information on at least six degrees of freedom that correspond to three translations, yaw, pitch and roll with respect to a point of the dorsum or palm of the hand. A position and orientation of each of the first and second sensors is detected. A first distance between said point and a metacarpal-phalangeal joint of the finger, a second distance between the melacarpal-phalangeal joint and a proximal interphalangeal joint, a third distance between the proximal interphalangeal joint and a distal interphalangeal joint, and a fourth distance between the distal interphalangeal joint and the first sensor are measured. A position and orientation of each of the three joints is calculated on the basis of the measured first to fourth distances, the detected position and orientation of the first sensor, and the detected position and orientation of the second sensor.

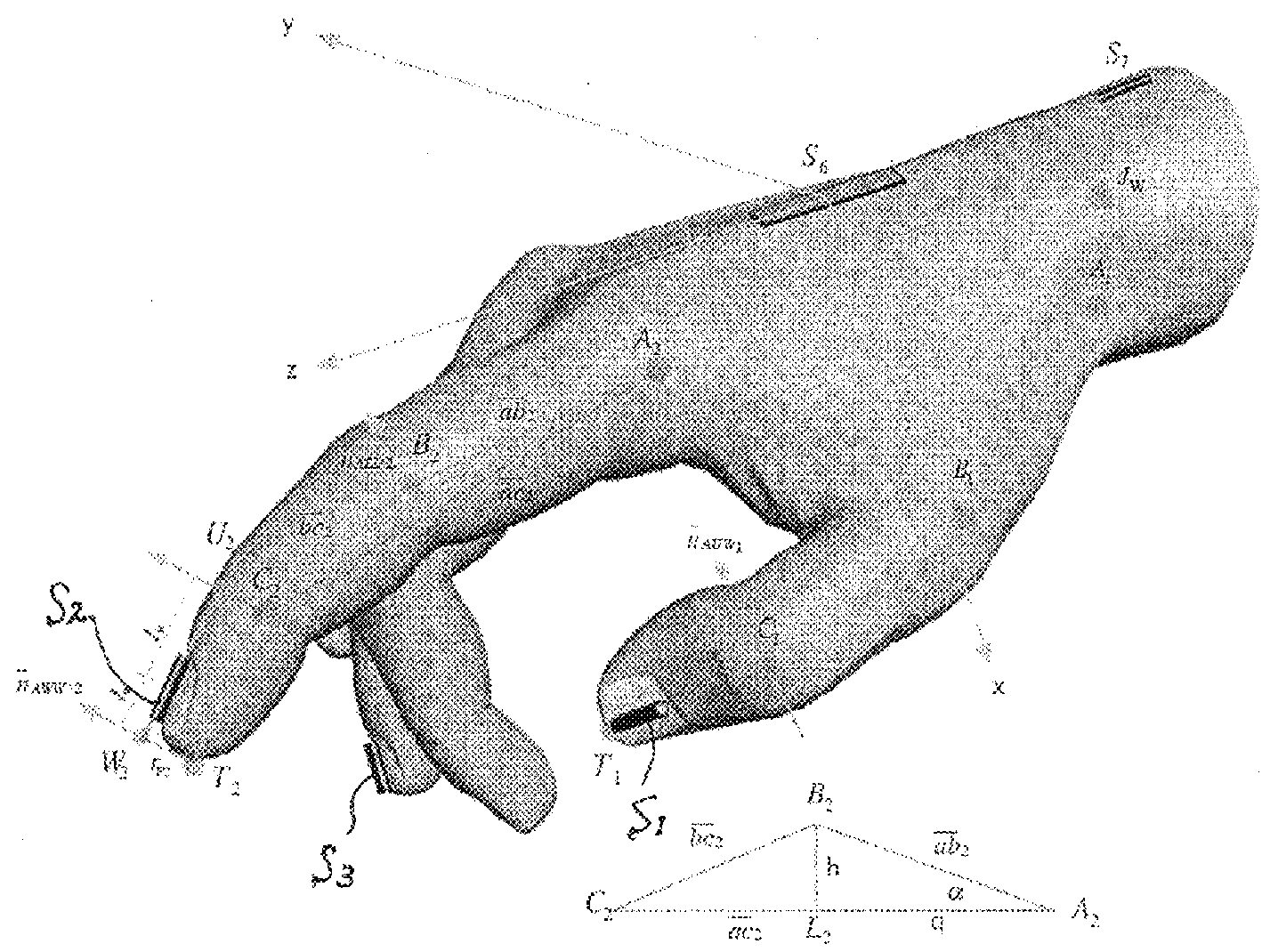


Fig. 1

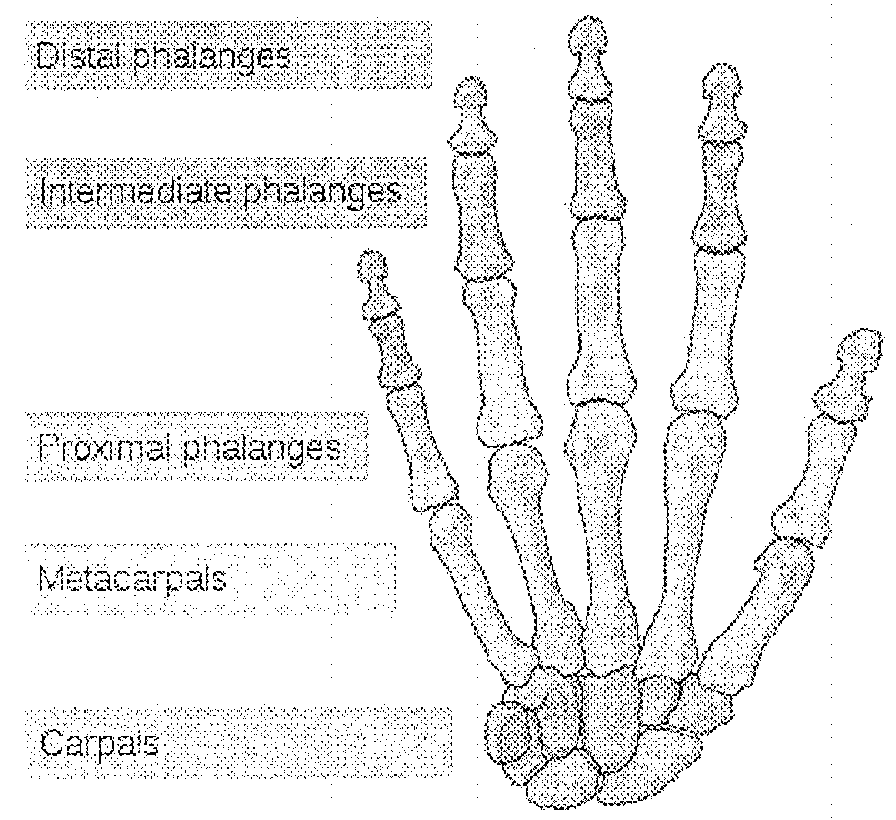

Fig. 2

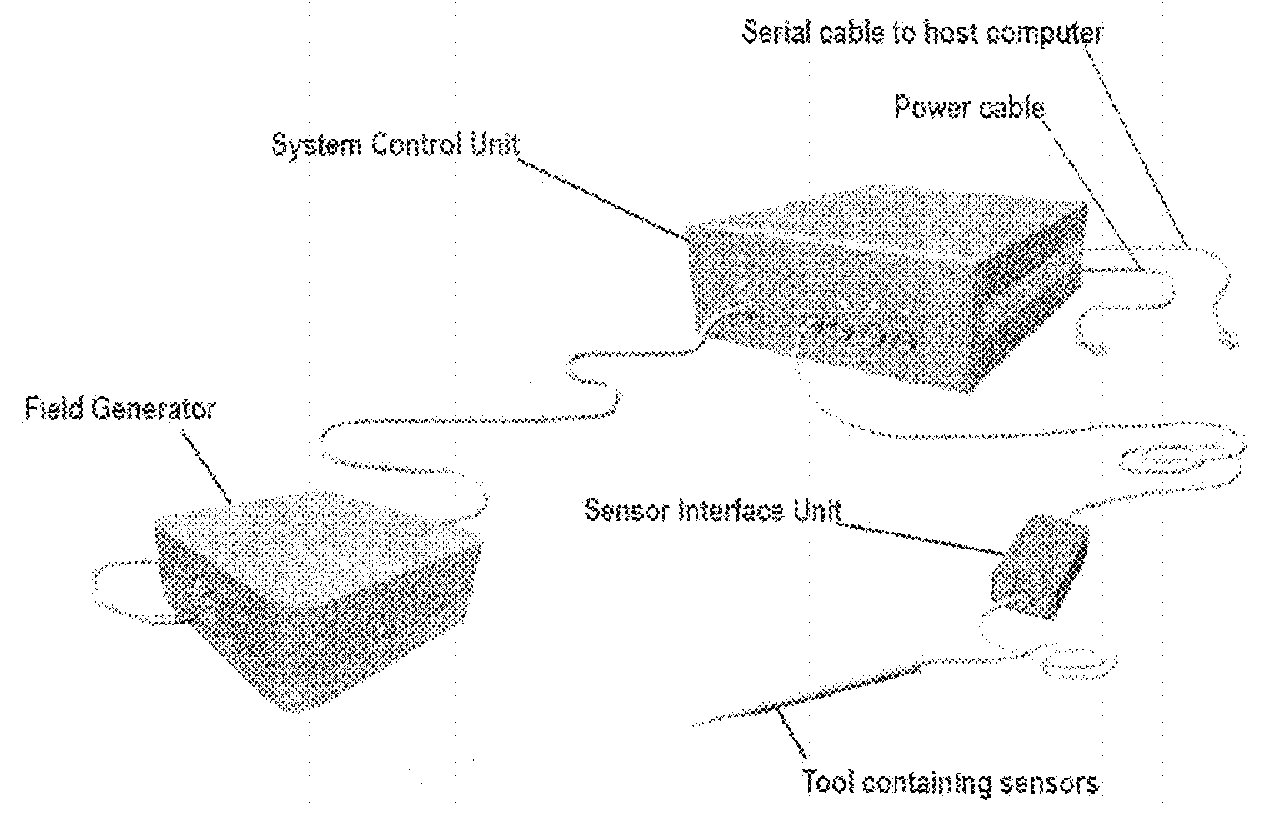




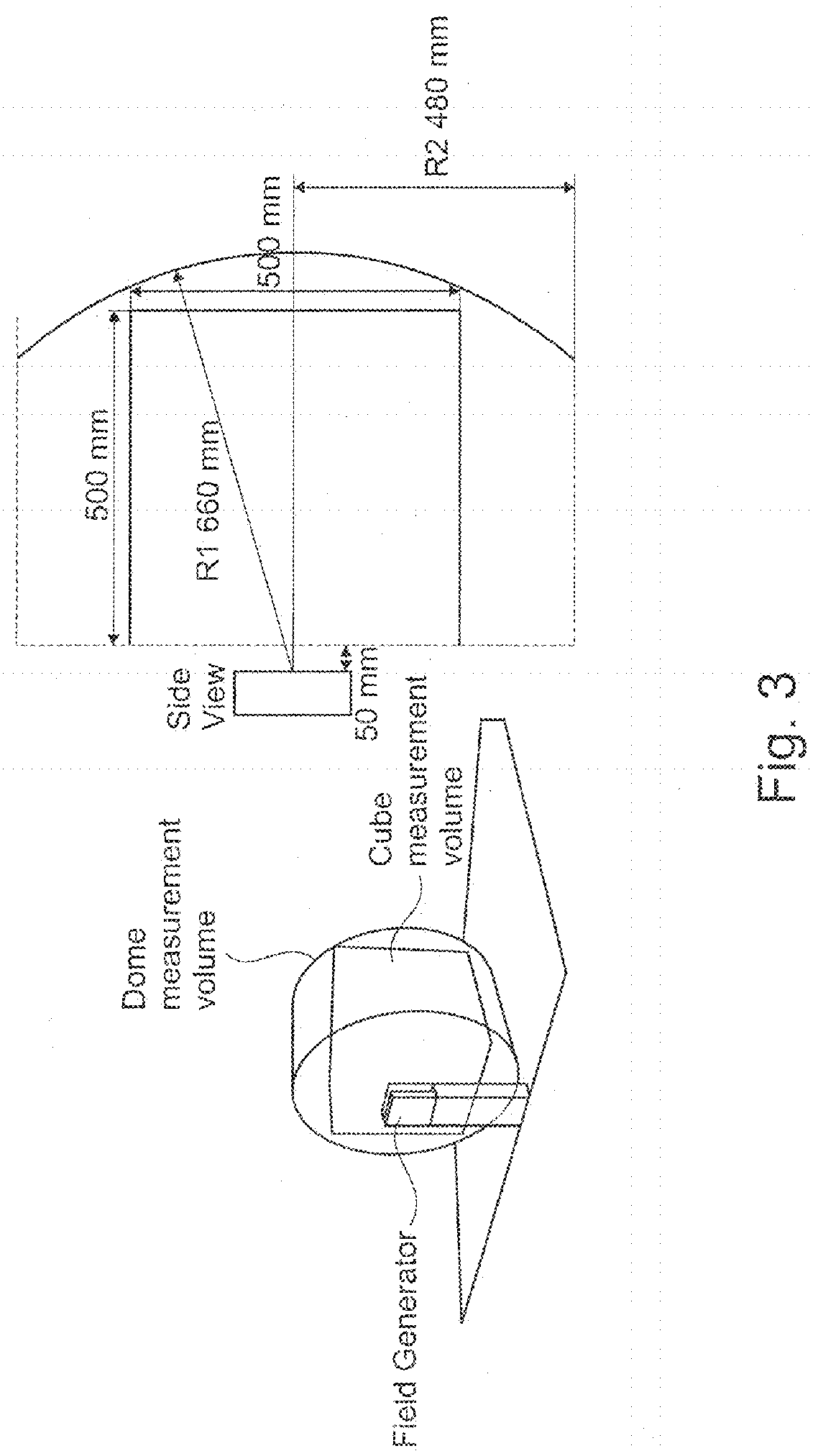


Fig. 4

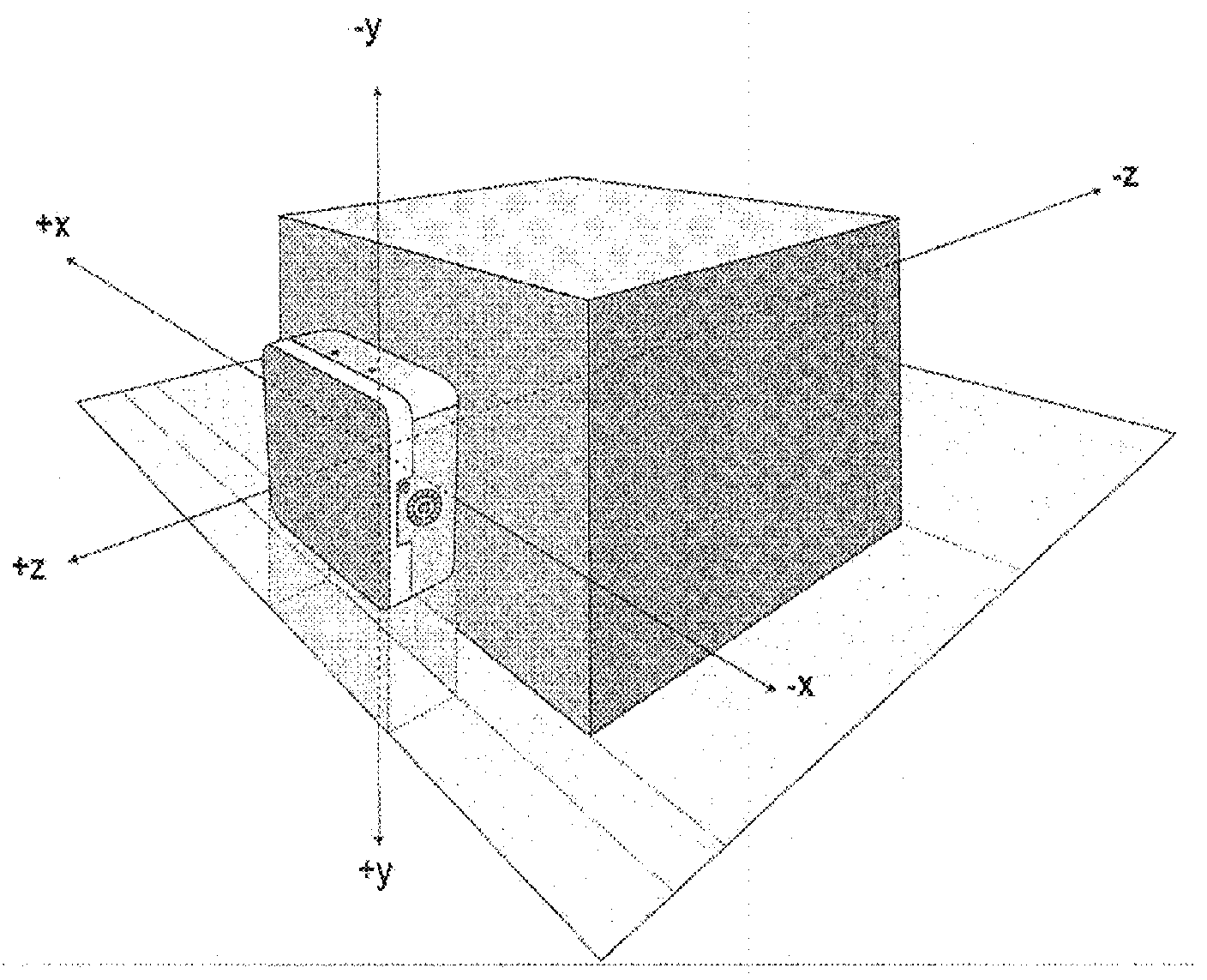


Fig.

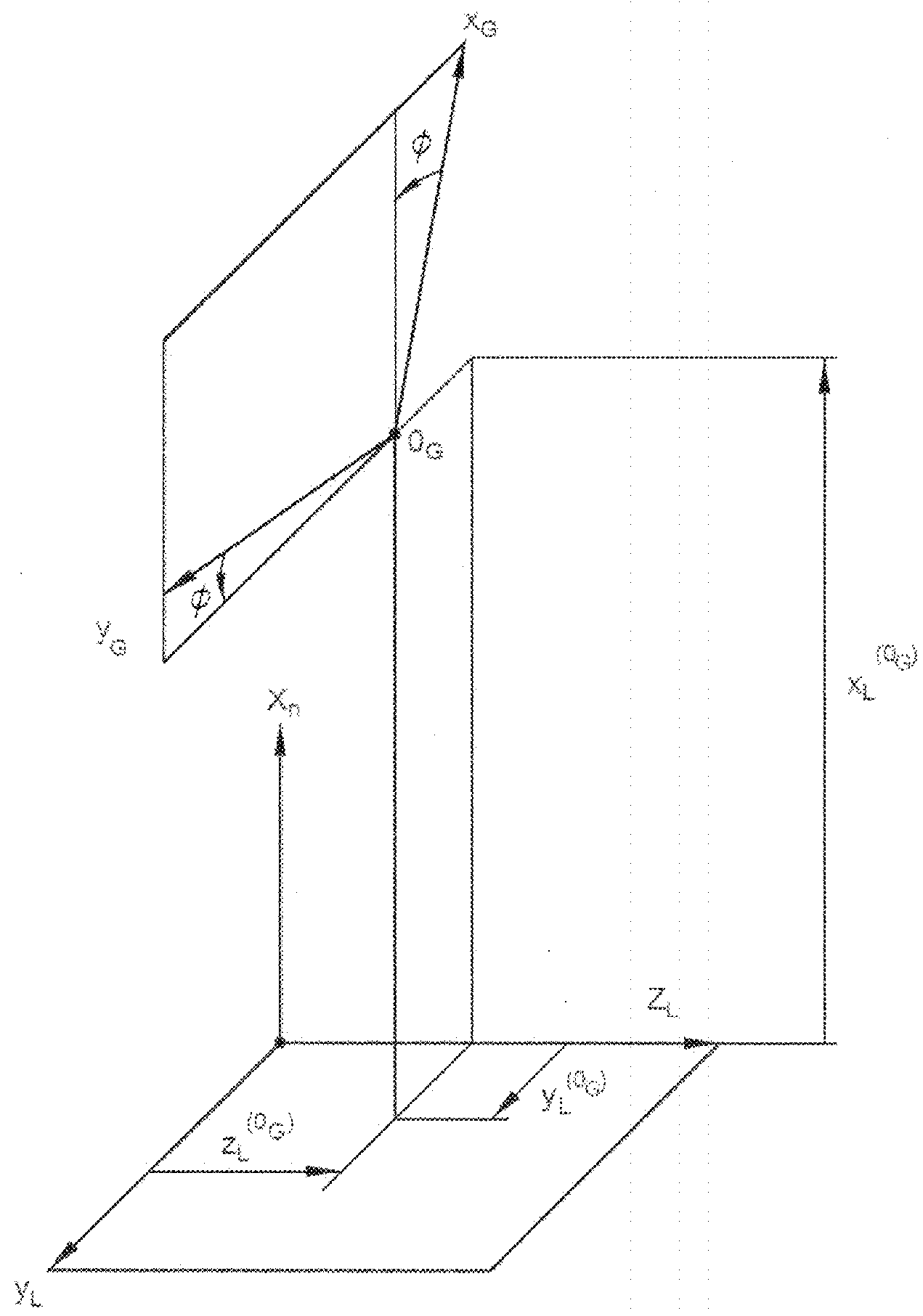




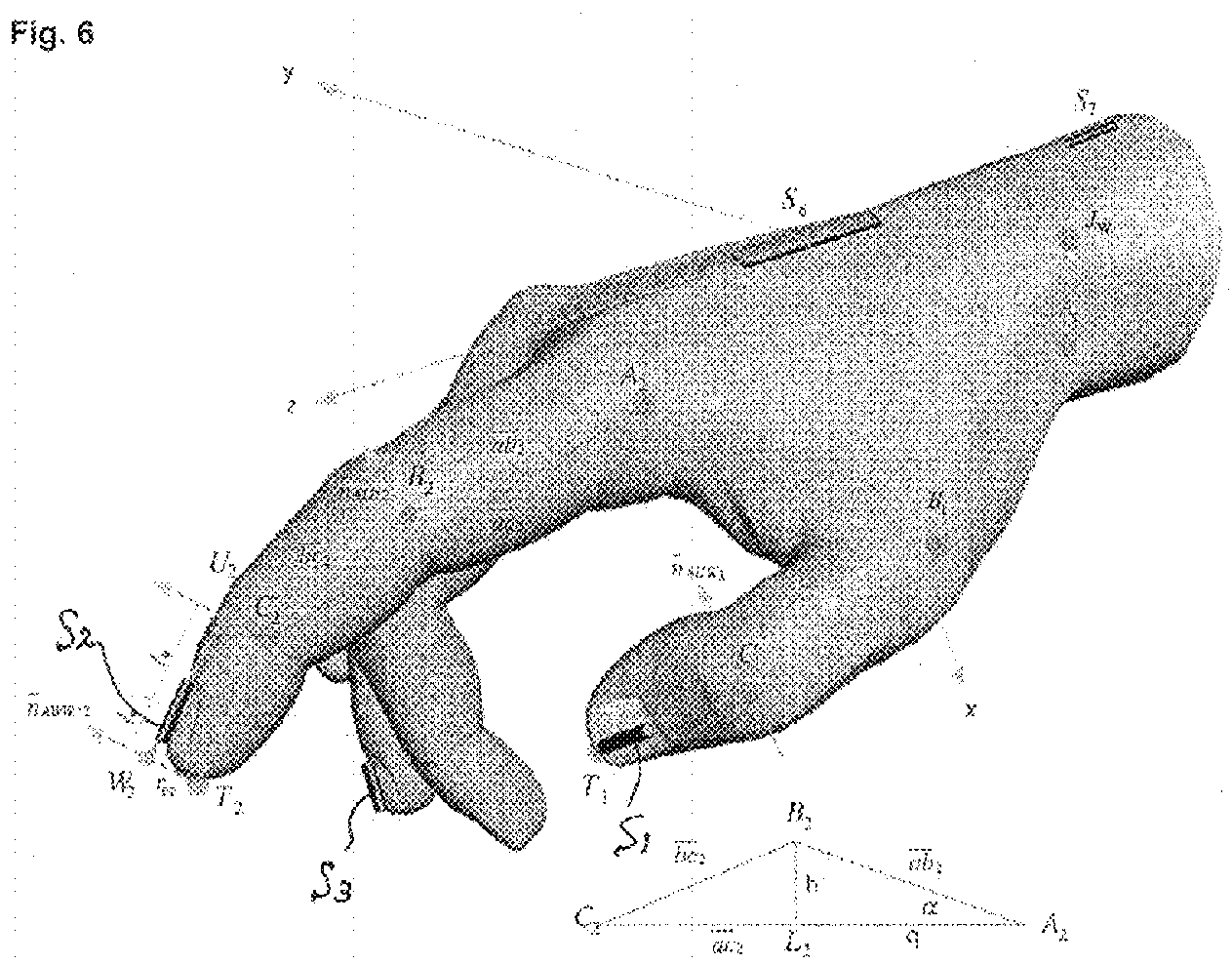

Fig. 7

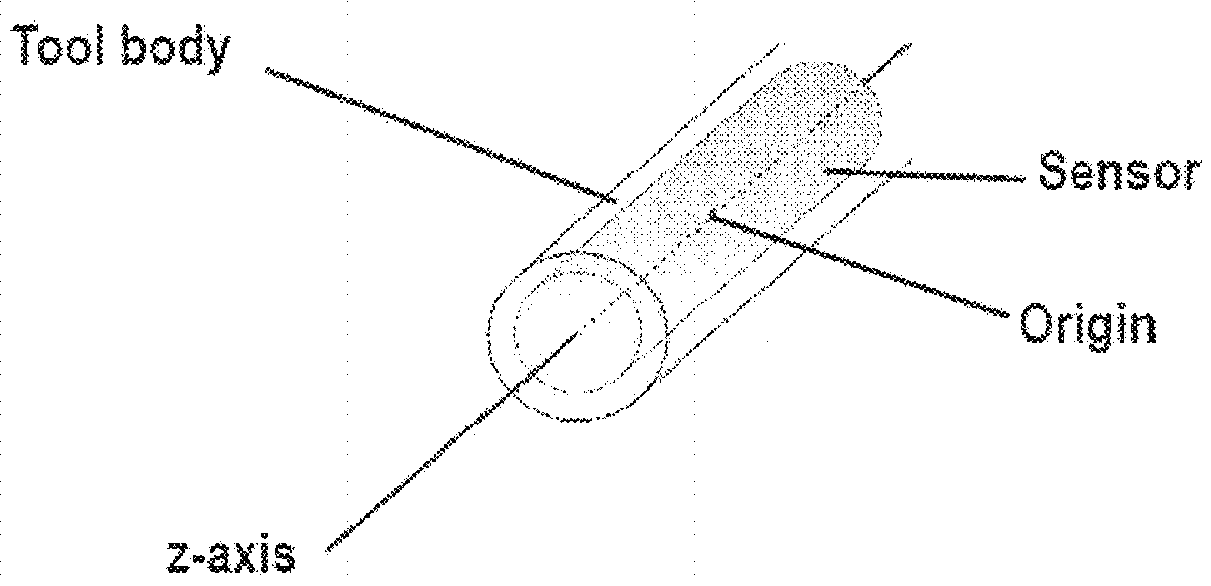


Fig. 8

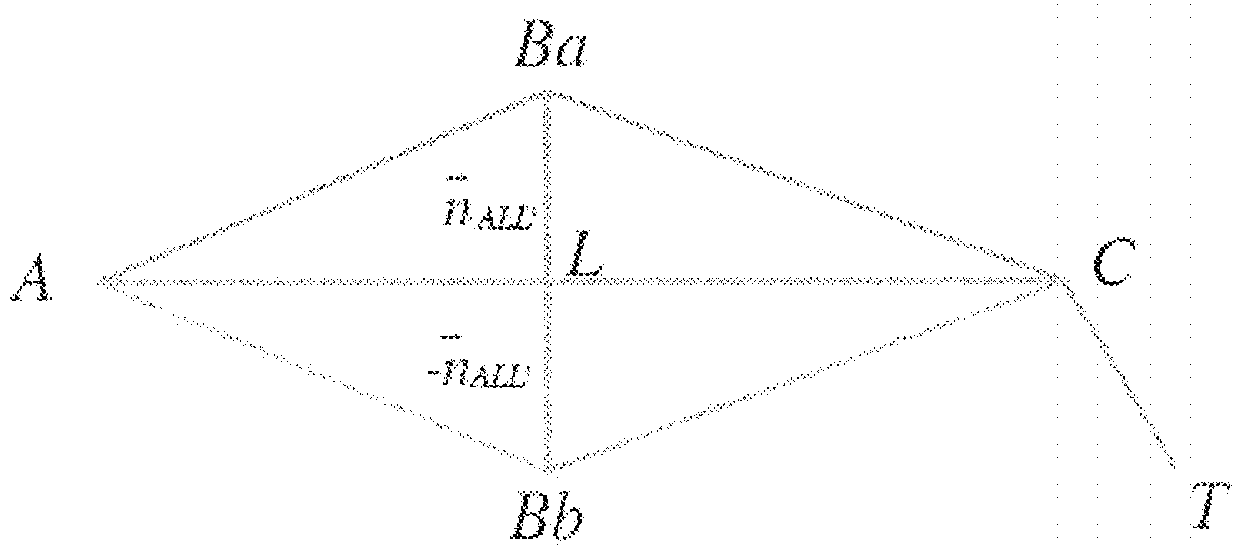



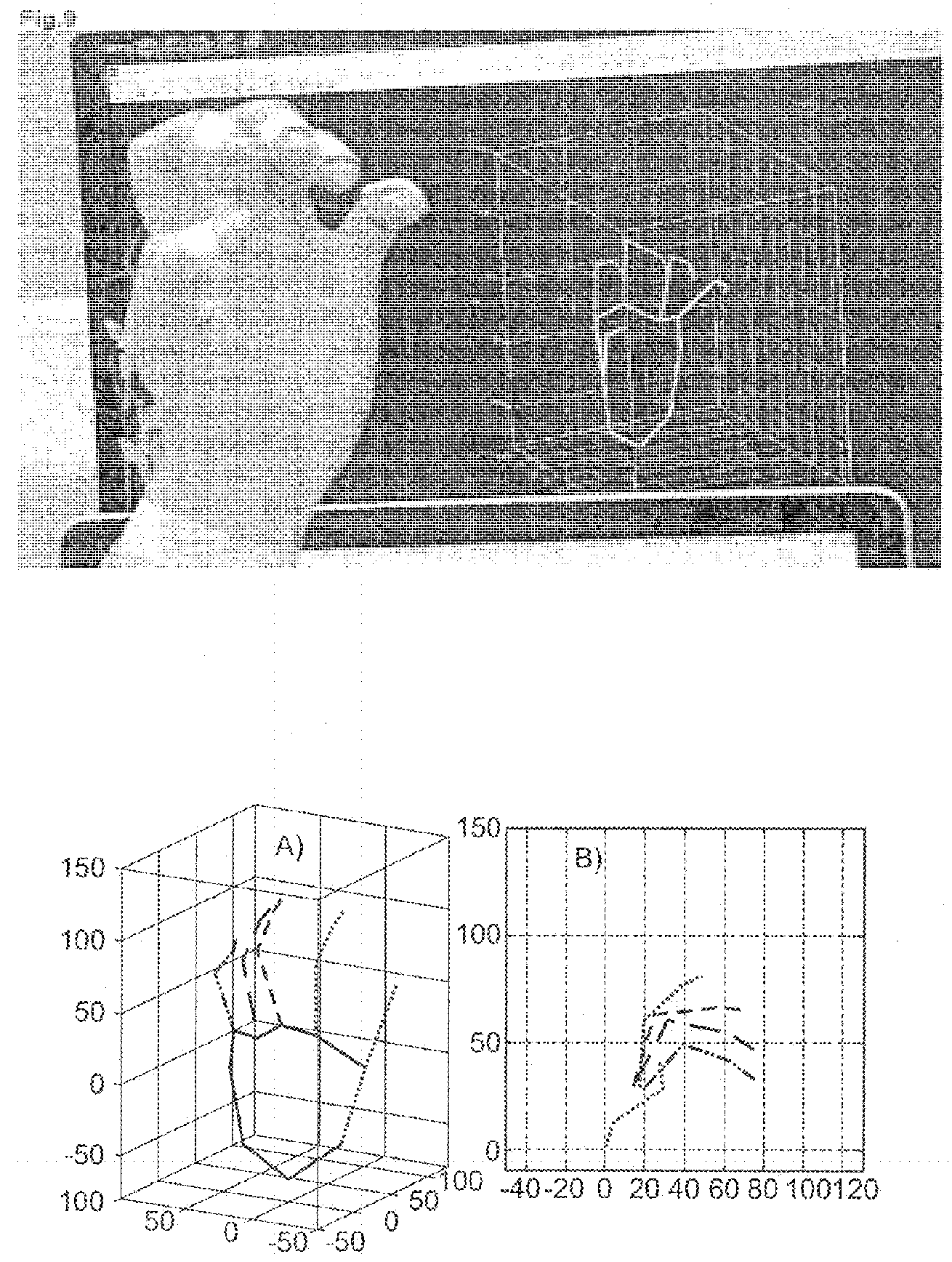

Fig. 10 


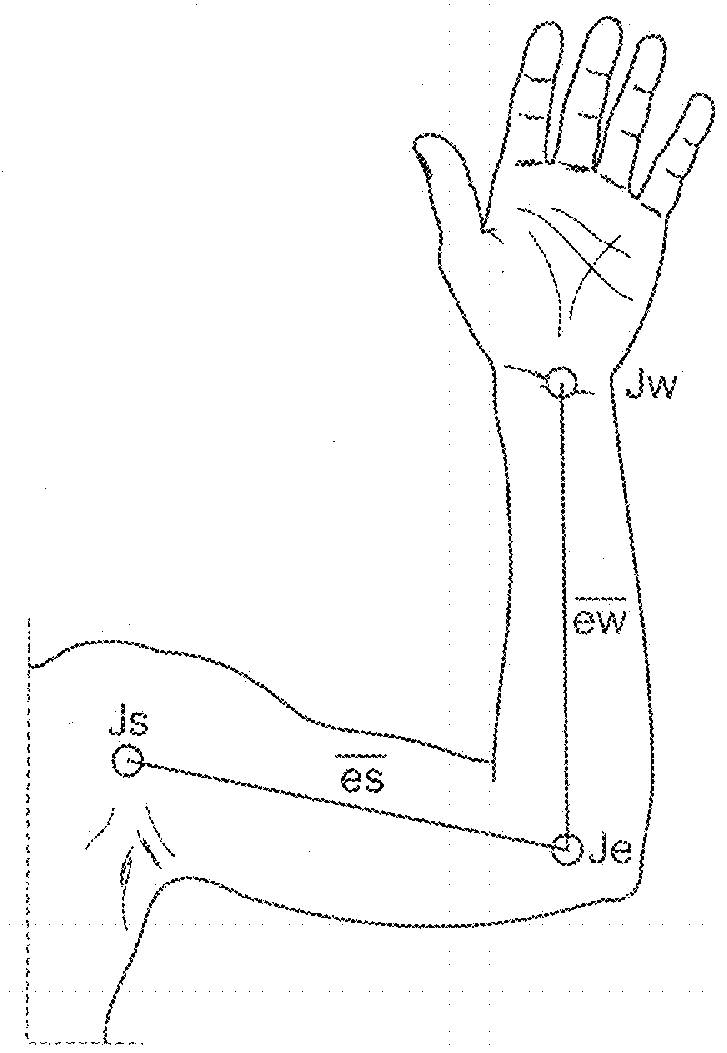

Fig. 11

Fig. 32

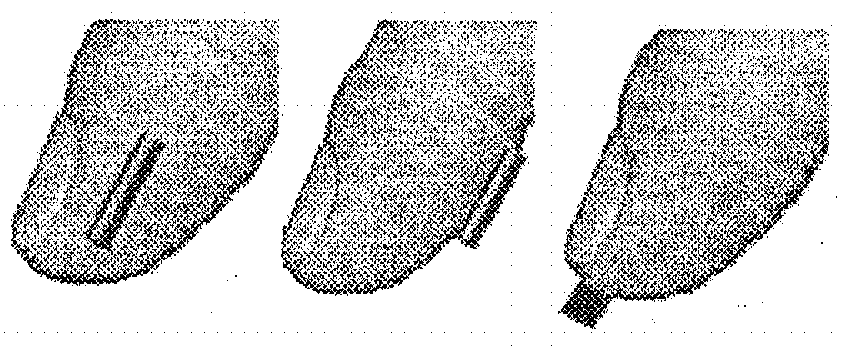


Fig. 13

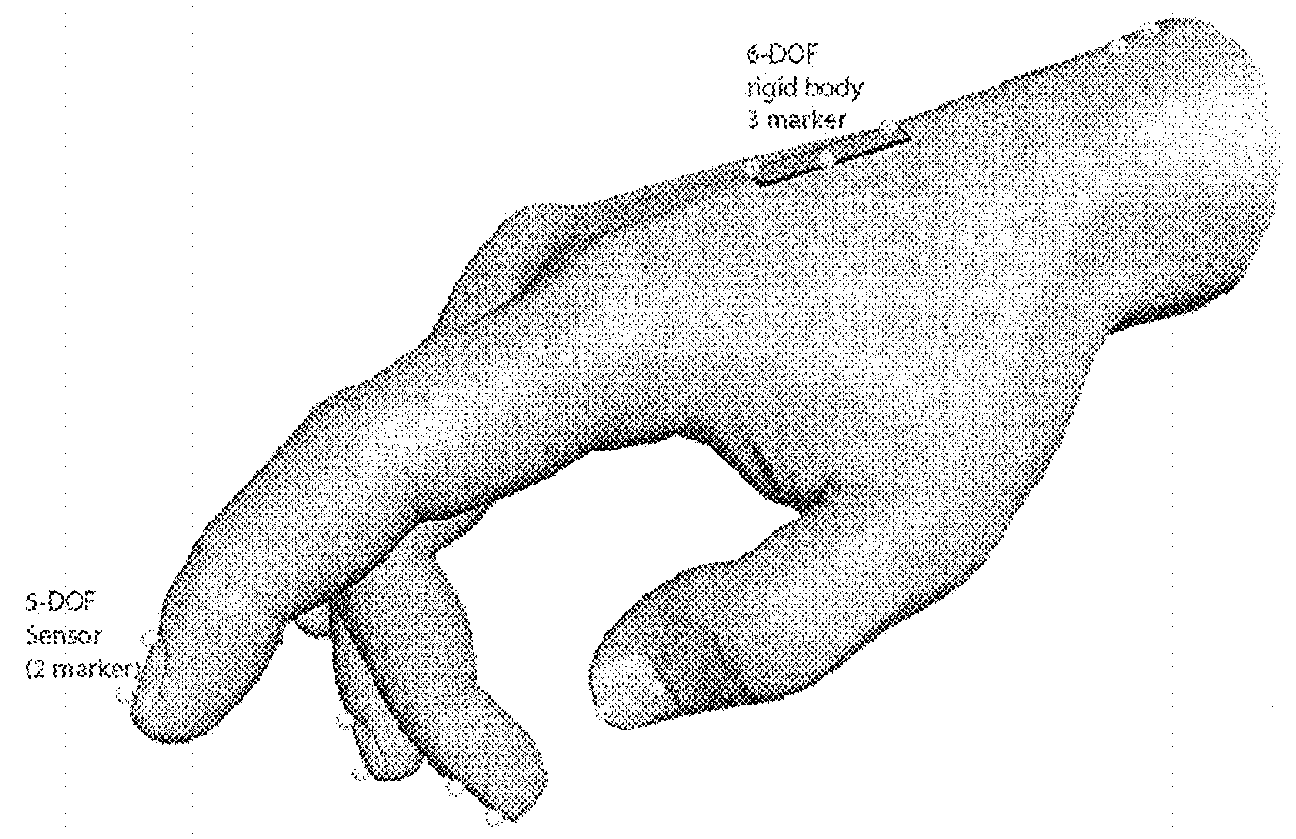




\section{MODELLING OF HAND AND ARM POSITION AND ORIENTATION}

\section{TECHNICAL FIELD}

[0001] The present invention relates to a method and system for modelling a position and orientation of a primate hand and arm.

\section{BACKGROUND OF THE ART}

[0002] Finger movements are highly complex. Therefore, humans spend much of their childhood learning to use their hands. I Iolding and moving objects of different size, shapes and weight is part of our early life, but the biomechanic system enabling these movements is highly sophisticated. The human hand as well as the hand or macaque monkeys are able to use 29 degrees of freedom. This fact and the wide range of motion (sec Table below) allow primates to perform complex grasp types.

\section{TABLE}

\begin{tabular}{|c|c|c|}
\hline \multicolumn{3}{|c|}{$\begin{array}{l}\text { Range of motion of a human hand (C. Youngblut, R. E. Johnston } \\
\text { S. H. Nash et al., "Review of virtual environment } \\
\text { Interface tcchnology," I.f.D. Analyses, ed., 1996) } \\
\text { (MCP: metacarpal-interplalangeal joint, PIP: proximal- } \\
\text { intcrphalangcal joint, IP: interphalangeal-ioint) }\end{array}$} \\
\hline Joint & Motion & Range \\
\hline \multirow[t]{5}{*}{ Thumb } & Palmar Adduction & $90^{\circ}$ \\
\hline & Radial Abduction & $80-90^{\circ}$ \\
\hline & Opposition & $90^{\circ}$ \\
\hline & MCP Flexion & $50^{\circ}$ \\
\hline & PIP Flexion & $80^{\circ}$ \\
\hline \multirow[t]{9}{*}{ Digits } & Abduction/Adduction & $\pm 15^{\circ}$ \\
\hline & Index MCP Flexion & $86-90^{6}$ \\
\hline & Index MCP Extension & $22-45^{\circ}$ \\
\hline & Index IP Flexon & $100-110^{\circ}$ \\
\hline & 2nd finger $\mathrm{MCP}$ & $91^{\circ}$ \\
\hline & Flex. & \\
\hline & 2nd finger MCP Ext. & $18^{\circ}$ \\
\hline & 2nd finger IP & $105^{\circ}$ \\
\hline & Flexion & \\
\hline
\end{tabular}

[0003] The human hand consists of 27 bones as illustrated in FIG. 1. The carpus account for 8 , the metacarpus or palm contains five; the remaining fourteen are digital bones: fingers and thumb. The joints connecting them are named meracarpal-phalangeal joints, proximal-interphalangeal joints and distal-interphalangeal joints. The only movements permitted in the interphalangeal joints are flexion and extension, whereas the metacarpal-phalangeal joint is also able to perform adduction and abduction. The most versatile finger is the thumb, which is capable of bending in sucb a way that it can touch all the other digits on the hand. The opposable thumb is reserved to the primate species.

[0004] There have been known various tracking systems for tracking a position and/or an orientation of an orientation such as hand.

[0005] For example, a glove for measuring hand kinemalies is known, wherein a flex sensor in the form of a strip adapled to change their impedance in proportion to the strain are provided, so that the angle of the finger joint can be evaluated (Simon A. Overduin et al. "An instrumented glove for small primates", Journal of Neuroscience Methods, 187 (2010) p100 to 104). However, such a strip has to be provided under and above each joint. It is to be noted that this glove provides the angle values but does not provide information on the positions in $3 \mathrm{D}$ space.

[0006] The object of the present invention is to provide a method and system for modelling a position as well as an orientation of a hand, i.e. the positions and orientations of the finger joints with as small a number of sensors as possible.

\section{DISCLOSURE OF THE INVENTION}

[00(07] In an aspect of one present invention, a method for modelling a position and orientation of a hand, includes the step of attaching at least a first sensor on a phalanx distalis of a finger. The first sensor is adapted to provide information on at least five degrees of freedom that correspond to three translations, yaw and pinch. A second sensor is placed at a fixed position relative to a dorsum or palm of the hand. The second sensor is adapted to provide information on at least six degrees of freedom than correspond to three translations, yaw, pitch and roil with respect to a point of the dorsum or palm of the hand. $\Lambda$ position and orientation of each of the first and second sensors is detected. $\Lambda$ first distance between said point and a metacarpal-phalangeal joint of the finger, a second distance between the metacarpal-phalangeal joint and a proxinnal interphalangeal joint, a third distance between the proximal interphalangeal joint and a distal interphalangeal joint, and a fourth distance between the distal interphalangeal joint and the first sensor are measured. A position and orientation of each of the three joints is calculated on the basis of the measured first to fourth distances, the delected position and orientation of the first sensor, and the detected position and orientation of the second sensor.

[0008] In one embodiment, the first sensor has a sensor coil with its axis being placed along a longitudinal direction of the finger. The second sensor has two sensor coils with their axes being placed in a geometrically defined position to each other so that the second sensor provides information on a roll of said point. A varying magnetic field is generated so that the first and second sensors induce voltages, so that at the detection step the position and orientation of each of the first and second sensors is detected cased on the induced voltages.

[0009] To simplify the construction, the second sensor is preferably arranged on the dorsum or palin of the hand.

[0010] In a further aspect of the present invention, a system for modelling a position and orientation of a hand includes a glove with glove fingers and a modelling deviec. The glove includes at least a first sensor on a portion of the glove finger corresponding to a phalanx distalis of a finger. The first sensor is adapted to provide information on at least five degrees of freedom that correspond to three translations, yaw and pitch. The glove is also provided with a second sensor at a position that is fixed on a portion of the glove finger corresponding to a dorsum or palm the hand. The second sensor is adapted to provide information on at least six degrees of freedom that correspond to three translations, yaw, pitch and roll with respect to a point of the dorsum or palm of the hand. T'be modelling device includes a detector for detecting a position and orientation of each of the first and second sensors. The modelling device also includes a storage for storing a first distance between said point and a first joint of the glove finger corresponding to a metacarpal-phalangeal joint of the finger, a second distance between the first joint and a second joint of the glove finger corresponding to a proximal interphalangeal joint of the finger, a third distance between the second joint and a third joint of the glove finger corresponding to a distal 
interphalangeal joint of the finger, and a fourth distance between the third joint and the first sensor. The modelling device is further provided with a calculator for calculating a position and orientation of each of the three joints on the basis of the stored first to fourth distances, the detected position and orientation of the first sensor, and the detected position and orientation of the second sensor.

[0011] According to the present invention, it is possible to model a position and orientation of a hand preferably without placing additional sensors to detect the positions and orientations of the metacarpal-phalangeal joint, the proximal interphalangeal joint, the distal interphalangeal joint of the finger.

\section{BRIEF DESCRIPTION OF DRAWINGS}

[0012] FIG. 1 shows a human hand and its 27 bones; [0013] FIG. 2 shows a measuring part of a modelling system according to a first embodiment of the present invention; [0014] FIG. 3 shows an exemplary measurement volume within which a hand is to be placed;

[0015] FIG. 4 shows a coordinate system referenced to the field generator and a cubic measurement volume;

[0016] FIG. 5 shows a coordinate transformation;

[0017] FIG. 6 shows first sensors and a second sensor placed on a hand, joints and vectors used for hand-modelling; [0018] FIG. 7 is a perspective view of a 5 DOF (degrees of freedom) magnetic sensor;

[0019] lil(j. 8 shows a drawing to explain a calculation according to the first embodiment;

[0020] FIG. 9 shows an image of a real hand with sensors fixated with tapes and a modelled hand displayed on a monitor;

[0021] FIG. $10 \Lambda$ shows a perspective view of the modelled hand;

[0022] FIG. 10B shows a side view of the modelled hand; [0023] FIG. 11 shows sensors placed on an arm, joints and vectors used for arm-modelling aceording so a third embodiment of the present invention;

[0024] FIG. 12 shows other examples of sensor placement; and.

[0025] FIG. 13 shows an example of sensor placement for optical tracking technologies.

\section{BEST MODES FOR IMPLEMENTING THE INVENTION}

[0026] With reference to the drawings, preferred embodiinents of the preserve invention will be explained below.

\section{First Embodiment}

\section{Configuration}

[0027] Referring to FIG. 2, the modelling system of the present embodiment includes a tool having a plurality of sensors to be attached to the finger and a separate modelling device, and uses an electro-magnetic principle to track hand and finger movements. In other words, the sensors to be placed on the fingers are sensor coils to which a varying magnetic field is applied and a resnltant voltage is outputted to the modelling device.

[0028] As one example of such a modelling systen a Aurora measurement system developed by Northern Digital Inc., Waterloo, Canada may be used. The measurement system may have a sampling rate of $40 \mathrm{~Hz}$ per sensor. $\Lambda$ serial interface (Recommended Standard 232) may be used to transfer data from the system to a host computer with a maximum data rate of $115 \mathrm{kBaud}$.

[0029] The modelling system includes a field generator, a system control unit (SCU), sensor coils and a host computer (not shown), and may further include a sensor interface unit (SIU). The SCU or other power source provides power to the field generator, which in turn produces a series of varying inagnetic fields, creating a predeternined volume of varying magnetic flux. Sensors connected to the SCU, via the SIU, are induced by the varying magnetic fields of the field generator. The SIU converts the voltages induced by the sensors, into digital data that is processed by the SCU to calculate the position and orientation of each of the sensors. (On reqnest of the host computer, a package including information of all the sensors may he prepared and sent over a device interface (RS 323 ) to the host computer.

[0030] Instead, the sensors or SIU may be provided (or connected) wish a wireless transmitter for transmitting a signal corresponding to the measured voltages or the calculated position and orientation of each of the sensors to the SIU or SCU (receiver), respectively, for further processing. Such a configuration may be preferably implemented in a system (e.g. game system) in which the movement of the hand/finger is displayed on a monitor.

[0031] 'The field generator produces, for example, a cnbic measurement volume (e.g. $500 \mathrm{~mm} \times 500 \mathrm{~mm} \times 500 \mathrm{~mm}$ ) or a dome $(\mathrm{R} 1=660 \mathrm{~mm}, \mathrm{R} 2=480 \mathrm{~mm})$, as shown in FIG. 3 .

[0032] In the present embodiment the sensor coil is an AUROR sensor coils by Northern Digital Inc. In one example, the sensor coil has a diameter of $0.5 \mathrm{~mm}$ and a length of $8 \mathrm{~mm}$.

[0033] The modelling system of the present embodiment includes six magnetic sensors (five first sensors and a second sensor).

[0034] A sensor coil (each constituting the first sensor) is placed on a phalanx distalis of each of the digits and the thumb to provide 5 DOF (degrees of freedom) information: the Cartesian coordinates ( $\mathrm{x}, \mathrm{y}, \mathrm{z}$ ) (three translations) as well as the yaw angle and the pitch angle of the sensor (in FlG. 6 three sensor coils $S_{1}, S_{2}, S_{3}$ of the five are shown). I'or this purpose, the axis of the coil of the first sensor is placed along a longitudinal direction of the finger. It is noted that since roll information of each finger around the finger axis is unnecessary (because the fingers are rotated together with the remaining part of the hand; this roll information is detected by a second sensor as explained below), a sensor providing 5 DOF information is sufficient.

[0035] On other hand, two sensor coils constituting the second sensor are placed on the dorsum so as to provide 6 DOF information: the Cartesian coordinates $(\mathrm{x}, \mathrm{y}, \mathrm{z})$ (three translations) as well as the yaw angle, the pitch angle and the roll angle of a point of the dorsum. That is, the second sensor provides information on a hand roll angle. For this purpose, the axes of the two sensor coils are placed in a geometrically defined position (e.g. orthogonal to each other; it is possible to obtain the roll information unless the axes are parallel to each other) so that the second sensor provides information on a roll of a point of the dorsum.

[0036] Together the two sensor coils present a plane in space that can be rotated in all directions around the abovementioned point (which is an origin of the local coordinate system $\mathrm{CV}$ of the second sensor). 
[0037] The two sensor coils are tracked separalely, but the outputs are processed by the SCU to determine the $6 \mathrm{DOF}$ information of the above-mentioned point). For this process the SCU will use a geometrical relationship of the two sensor coils saved in a memory-chip (SROM, serial read only memory) inside the SIU connector (FIG. 2). The geometry of the two sensor coils can be determined by a user. After the user has entered the needed information in a graphical user interface of the modelling system, a file is created. 'I'his file can be written to the SROM chip over a serial interface. During the initialization of the sensor coils the SROM is read to identify the origin.

\section{Hand-Modelling}

[0038] All calculations essential for hand-modelling are realized within the hardware configuration of the modelling system. The oblained position and orientation of the sensor will be referred to the coordinate system of the field generator (see FIG. 4).

[0039] To compute and track one's hand, a new local reference point at the dorsum (backside of the hand's wrist) is defined (FIG. 5). For this reason the 6 DOF sensor (second sensor) will be placed there. The dorsum of the hand is the best location for the reference sensor because the relative distance to the metacarpal phalangeal joints does not change. The importance of this condition will become clearer in the following calculations.

[0040] It is to be noted that the $6 \mathrm{I})(\mathrm{)})$ i sensor may be arranged on a palm of the hand instead of on the dorsum. l'urthermore, the second sensor may not necessarily be placed directly on the dorsum or palm (i.e. the second sensor is spaced a distance away above the dorsum or below the palm) because the distance between the second sensor and a eertain point of the dorsum or palm is constant, so that using the 6 DOF information of the sensor, the position of said point on the dorsum or palm and the orientation of the hand (the orientation of the second sensor is identical to that of the hand even if the second sensor is spaced away from the dorsum or palm) can still be obtained.

[0041] Here the field generator's coordinate system is called a global coordinate system while the hand's coordinate system a local coordinate system $C_{L}$. Point $S$ is represented in coordinate system $C_{G}$ by the position vector

$$
r_{G}=\left[x_{G}, y_{G}, z_{G}, 1\right]^{T}
$$

[0042] The same point $\mathrm{S}$ can be determined in coordinate system $\mathrm{C}_{L}$ by the position vector

$$
r_{t},\left|x_{t}, y_{t,}, z_{t,}, 1\right|^{T}
$$

with the Matrix equation

$$
\mathrm{r}_{L}=\mathrm{M}_{L G^{\mathrm{r}}} \mathrm{s}
$$

[0043] Matrix $\mathrm{M}_{L G}$ is represented by

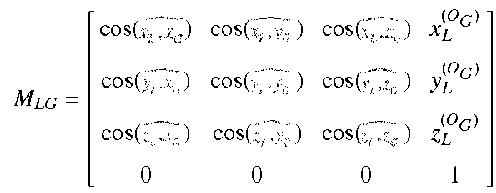

$\mathrm{O}_{G}$ and $\mathrm{O}_{L}$ are the origins of the "global" and the "local" coordinate system (FIG. 5); subscript LG designation $\mathrm{M}_{L G}$ indicates that the transformation is performed from $\mathrm{C}_{G}$ to $\mathrm{C}_{L}$. [0044] In this way the global coordinates from the sensors $\left\{\mathrm{S}_{I_{1},}, \mathrm{~S}_{I_{2}}, \ldots, \mathrm{S}_{I_{5}}\right\}$ mounted on the phalanx distalis of digits and thumb are transformed to the local coordinate system of the reference sensor $\mathrm{s}_{G_{i}}\left[\mathrm{x}_{G_{i}}, \mathrm{y}_{G_{i}}, \mathrm{z}_{G_{i}}, 1\right]^{T}$.

$$
\mathrm{s}_{L_{j}}=\mathrm{M}_{L G^{\prime}} \mathrm{s}_{G_{i}} \forall i=\{1,2, \ldots, 5\}
$$

[0045] From now on equations refer to the local coordinate system. Therefore subscripts indicating the coordinate frame are omitted.

[0046] The whole hand can be described by the wrist-joint $\left(\mathrm{J}_{w}\right)$, the five metacarpal-phalangeal joints $\left\{\mathrm{A}_{1}, \mathrm{~A}_{2}, \ldots, \mathrm{A}_{5}\right\}$, the five proximal-inkerphalangeal joints $\left\{B_{1}, B_{2}, \ldots, B_{5}\right\}$ and the distal-interphalangeal joints $\left\{\mathrm{C}_{1}, \mathrm{C}_{2}, \ldots, \mathrm{C}_{5}\right\}$ as shown in FIG. 6. Each of these variables represents the joint's local coordinates $[\mathrm{x}, \mathrm{y}, \mathrm{z}]^{T}$

[0047] As mentioned before, the distance between $S_{6}$ (origin of the local coordinate system of the second sensor) and the metacarpal-phalangeal joints are assumed to be constant. Therefore $\left\{A_{1}, A_{2}, \ldots, A_{5}\right\}$ can be determined by measuring their distances to $\mathrm{S}_{6}$.

[0048] With the given transformed points $\left\{\mathrm{S}_{L_{1}}, \mathrm{~S}_{L_{2}}, \ldots\right.$, $\left.S_{L_{5}}\right\}$ and the measured points $\left\{\Lambda_{1}, \Lambda_{2}, \ldots, \Lambda_{5}\right\}$ the remaining points $\left\{\mathrm{C}_{1}, \mathrm{C}_{2}, \ldots, \mathrm{C}_{5}\right\}$ and $\left\{\mathrm{B}_{1}, \mathrm{~B}_{2}, \ldots, \mathrm{B}_{5}\right\}$ can be calculated:

[0049] For this, a third coordinate system $\mathrm{C}_{S}$ is determined, which is the local coordinate system of the first sensor, having an origin in its center as illustrated in FIG. 7. X and Y-axes of the 5 DOF sensors coordinate system are not determined. Because transformations were only done in the 2-axis this limitation can be ignored

[0050] 'Iwo points along the sensor's 7-axis are introduced according to Fili. 6.

$$
\begin{aligned}
& U_{s}=\left[0,0,-l_{U}\right]^{T} l_{U} \ldots \text { distance from origin to } U \\
& \mathrm{~W}_{t}=\mid\left(0,0,\left.\left.\right|_{w}\right|^{T} \mathrm{I}_{w} \ldots \text { distance from origin tos } \mathrm{W}\right.
\end{aligned}
$$

[0051] These points are then transformed to the hand's local coordinate system $\mathrm{C}_{L}$.

$$
\begin{aligned}
& \mathrm{U}_{L_{i}}=\mathrm{M}_{\iota, s} \mathrm{I}_{S_{i}} \forall \mathrm{i}=\{1,2, \ldots, 5\} \\
& \mathrm{W}_{L_{i}}=\mathrm{M}_{L S} \mathrm{~W}_{S_{j}} \forall \mathrm{i}=\{1,2, \ldots 5\}
\end{aligned}
$$

$\left|\overrightarrow{u w}_{i}\right|$ does have the same length as the distal phalanx of finger i. Instead of having the a vector pointing from $U$ to $\mathrm{W}$, it would be more accurate for the following calculations to have the same vector pointing from $\mathrm{C}$ to $\mathrm{T}$; $\mathrm{T}$ is defined as the finger tip (FIG. 6).

[0052] l3ecause $A, U$ and $W$ are on one plane, the vector normal to this plane $\overrightarrow{\mathbf{n}}_{\text {AUW }}$ can be calculated.

$$
\vec{n}_{A U / W}=\frac{\overrightarrow{a u} \times \overrightarrow{u v}}{|\overrightarrow{a u} \times \overrightarrow{u v}|}
$$

[0053] A uew poiut $\mathrm{W}^{\prime}=\mathrm{W}+\overrightarrow{\mathrm{n}}_{\text {AIJW }}$ can be used to find the vector normal to the plane described by the points $\mathrm{A}$, $\mathrm{w}$ and $W^{\prime}$. This new vector $\overrightarrow{n_{A U W}}$ is orthogonal to $\overrightarrow{\mathrm{uw}}_{i}$ and is part of the plane $\Lambda, U, W$ and can be used to shift the points $U$ and $W$ parallel into the center of the finger to get the point $\mathrm{C}$ and $\mathrm{T}$. 


$$
\begin{aligned}
& T_{i}=W_{i}+\vec{n}_{A W W^{\prime \prime}} \cdot r_{F_{i}} \\
& C_{i}=U_{i}+\vec{n}_{A W W W^{\prime}} \cdot r_{F_{i}}
\end{aligned}
$$

$\mathrm{r}_{\bar{F}_{i}} \ldots$ radius of finger $\mathrm{i}$

[0054] The fact that the proximal-interphalangeal joints have only one degree of freedom allows to calculate point 13 in 31 )-space. In other words, point $\mathrm{A}, \mathrm{B}$ and $\mathrm{C}$, are always moving within the same plane defined by these three points. To get point $\mathrm{B}$ the triangle built by $\mathrm{A}, \mathrm{B}$ and $\mathrm{C}$ is first calculated in 2D-space. The points A, C as well the measured length $|\overrightarrow{a b}|$ (proximal phalanx) and $|\overrightarrow{b c}|$ (intermediate pha$\operatorname{lan} x$ ) are known. l'urthermore the distance $|\overrightarrow{a c}|$ can be calculated. Using the law of cosines the angle

$$
\begin{aligned}
& a=\arccos \left(\frac{|a b|^{2}+|\overrightarrow{a c}|^{2}-|b|^{2}}{2 \cdot|a \vec{b}| \cdot|\overrightarrow{a c}|}\right) \text { and } \\
& h=\sin (\alpha) \cdot|\overrightarrow{a b}| \\
& q=\cos (\alpha) \cdot|\overrightarrow{a b}|
\end{aligned}
$$

can be calculated (according to FIG. 6). Therefore point $\mathrm{L}$ is

$$
L=A+\overrightarrow{\mathrm{e}_{a c}} \cdot \mathrm{q}
$$

[0055] There are endless solutions for $B$, all of them orthogonal to $\overrightarrow{\mathrm{ac}}$ around point $\mathrm{L}$. To find the solution on the plane the normal vector $\overrightarrow{\mathrm{n}}_{A U W}$ is used to calculate $\mathrm{L}$ '.

$$
L^{\prime}=L+\vec{n}_{A U W}
$$

[0056] The vector normal to the plane of point A, L and L' is

$$
n_{A L^{\prime}}=\frac{\overrightarrow{a l} \times a l^{\prime}}{\left|\overrightarrow{a l} \times \overrightarrow{a l^{\prime}}\right|}
$$

[0057] Two solutions on plane Al l , can be found as illnstrated in li'I(j. 8.

$$
\begin{aligned}
& B_{a}-L_{i}+\vec{n}_{A L L_{i}^{\prime}} \cdot h \\
& B_{b}=L_{i}-\vec{n}_{A L L_{i}^{\prime}} \cdot h
\end{aligned}
$$

[0058] During natural finger movements the flexion of the intermediate phalanges are associated with the flexion of the distal phalanges. The same is true for extension. This fact can be used to find the anatomically correct solution for the intermediate phalangeal join position, (point B in FIG. 8). In other words, one solution (point $\mathrm{Ba}$ or $\mathrm{Bb}$ ) with a greater distance no the finger tip (point T in FIG. 3 ) is selected.

[0059] An operation of the modelling system will now be explained.

[0060] The first sensor is attached on a phalanx distalis of each finger while the second sensor is atlached on the dorsum of the hand.

[0061] The necessary information on the finger of a user is measured. Specifically, a first distance between a point (origin of the second sensor) on the dorsum of the hand and the metacarpal-phalangeal joint A of the finger, a second distance between the metacarpal-phalangeal joint $A$ and the proximal interphalangeal joint $B$, a third distance between the proximal interphalangeal joint $B$ and the distal, interphalangeal joint $C$, and a fourth distance between the distal interphalangeal joint $\mathrm{C}$ and the first sensor are measured. The second distance and the third distance may be measured before attaching the sensors.

[0062] Next, a varying magnetic field is generated so that the Voltages induced in the sensors are sent as digital data (generated by sheSIU) to the SC (U. On the basis of the digital data, the SCU detects a position and orientation of each of the first sensors and the second sensor at intervals (e.g. sampling rate of $40 \mathrm{~Hz}$ ). When receiving a signal from the SCU, the host computer calculates a position and orientation of each of the three joints on the basis of the measured first to fourth distances of each finger, the detected position and orientation of tine first sensor of each finger, and the detected position and orientation of the second sensor.

[0063] The calculated position and orientation of each of the metacarpal-phalangeal joint $\Lambda$, the proximal interphalangeal joint $B$, and the distal interphalangeal joint $C$ of each finger is supplied to a driver for driving the monitor, in addition to the detected position and orientation of each sensor. [0064] A model of the hand is displayed on the monitor on the basis of the supplied information. A region between the adjacent joints may preferably be displayed as a line connecting the joints, as shown in FIGS. 3 and $\mathbf{1 0 .}$

\section{Second Iimbodiment}

[0065] Next, a second embodiment of the present invention is explained. A modelling system according to the present embodiment includes a glove with sensors, instead of separate sensors to be attached to the hand.

[0066] Specifically, the glove has a first sensor on a portion of cach glove finger corresponding to a phalanx distalis of a finger, and a second sensor at a position that is fixed on a portion (which may preferably be located at a non-visible place inside the glove) of the glove finger corresponding to a dorsum or palm of the hand. The first and second sensors are identical to those in the first embodiment. In one example, the first sensor is provided on a glove portion corresponding to the fingernail.

[0067] The hardware configuration is similar to that of the first embodiment shown in FIG. 2.

[0068] I lowever, in the second embodiment, there is provided a storage (for example in the host computer) for storing a first distance between a point of the dorsum (the second sensor provides 6 DOF information on said point) and a first joint of the glove finger corresponding to a metacarpal-phalangeal joint of the finger, a second distanee between the first joint and a second joint of the glove finger corresponding to a proximal interphalangeal joint of the finger, a third distance between the second joint and a third joint of the glove finger corresponding to a distal interphalangeal joint of the finger, and a fourth distance between the third joint and the first sensor.

[0069] The SCU is adapted to detect a position and orientation of each of the first and second sensors based on the voltages from the sensors (i.e. digital data from the SIU).

[0070] The host computer is further adapted to calculate a position and orientation of each of the three joints of the glove fingers on the basis of the stored first to fourth distances, the detected position and orientation of each of the first sensors, and the detected position and orientation of the second sensor. 
[0071] The operation (calculation of the position and orientation of each of the three joints) of the modelling system of the present embodiment is similar to that of the first embodiment, and therefore the explanation thereof is onitted.

\section{Third Embodinnent}

\section{Arm-Modelling}

[0072] Referring to FIG. 11, a third embodiment of the present invention is explained. In the third embodiment, in addition to the hand-modelling an arm-modelling takes place. Namely, the positions and orientations of a shoulder joint Js, an elbow joint Je and a wrist joint Jw will be determined. These joints are assumed to be on the same plane. Also, the elbow joints Je and wrist joints Jw are preferably on a rotation axis of the forearm.

[0073] As explained below, an additional sensor (sensor coil) $S_{7}$ to be placed on a forearm is needed to calculate positions and orientations of the entire arm. The axis of the sensor coil is placed along a longitudinal direction of the forearm.

[0074] A calculation method to he performed by the host computer (FIG. 2) will now be explained.

[0075] Assuming the shoulder position to he constant (e.g. the user sits on a chair), the position of the shoulder joint Is is determined. The length of the forearm is measured before the arm modelling.

[0076] In the coordinate system $\mathrm{C}_{L}$ of the sensor $\mathrm{S}_{6}$, the position of wrist joint $\mathrm{J}_{W}$ is described by the measured $\mathrm{x}-\mathrm{y}$ and $z$-distanes from the wrist joint to the reference sensor that is the second sensor $\mathrm{S}_{6}$ (FIG. 6). Here they are called the distances $\mathrm{w}_{x}, \mathrm{w}_{y}$ and $\mathrm{w}_{z}$.

$$
. / w=\mid()+w x,\left(0+w y,()+\left.w z\right|^{T}\right.
$$

[0077] The point gets transformed to the global coordinate system:

$$
J w_{G}=M_{L G}{ }^{-1} \cdot J_{W^{\prime}}
$$

[0078] The additional sensor $S_{7}$ is used to determine a new coordinate system $C_{n}$, that has the orientation of $S_{7}$ and its origin at the wrist joint point Jw. 'The rotation-matrix $\mathrm{M}_{w}$ is therefore defined by the orientation of $S_{7}$ and the position of Jw.

[0079] The position of the elbow joint Je in the coordinate system $\mathrm{C}_{w}$ is:

$$
J e_{w}=[0,0,-l f a]
$$

[0080] where lfa is the length of the forearm. The position of the elbow joint $\mathrm{Je}$ is then transformed into the global coordinate system.

$$
J e_{G}=M_{w} \cdot e_{w}
$$

[0081] The orientation of the elbow joint Je is the same as that of the sensor $S_{7}$. It is noted that the roll angle of the sensor $\mathrm{S}_{7}$ is determined on the basis of the roll angle of the second sensor $\mathrm{S}_{6}$ (since the hand and the arm rotate together around the forearm axis).

[0082] As such, the host computer obtains the position of the shoulder joint Js as well as the position and orientations of the wrist joint Jw and the elbow joint Je. This may be further processed, for example, to display the arm model together with the hand model.

[0083] Although the additional sensor $S_{7}$ may be located at an arbitrary position at the forearm as long as the yaw and pitch of the forearm is calculated, it is preferable to locate the sensor $\mathrm{S}_{7}$ close to the sensor $\mathrm{S}_{6}$ because the field generator provides a limited range of magnetic field.

[0084] In the same manner as the second embodiment, a glove may be provided with the additional sensor $S_{7}$. In this case, the sensor $S_{7}$ will have to be located close to the sensor $S_{6}$.

[0085] Although in the present embodiment the sensor coil is used as the third sensor $\mathrm{S}_{7}$ that is the same as the first sensor, i.e. 5 DOF sensor, it is sufficient that the third sensor on the forearm provides information on $2 \mathrm{DOF}$ that correspond to yaw and pitch.

[0086] The modelling system and method of the present invention has been explained with reference to the preferred embodiments. However, it should be understood that various changes, substitutions and alternations may be made without departing from the scope of the invention as defined by the appended claims.

[0087] For example, in the first and second embodiments the first sensor (finger-sensor) is placed on the fingernail or on a glove portion corresponding to the fingernail. I lowever, the first sensor may he placed on a side of the phalanx-distalis, below the phalanx-distalis, or on a finger tip as shown in FIG. 12.

[0088] The first sensors may not necessarily be provided corresponding to all the fingers.

[0089] In the first to third embodiments, sensor coils for electro-magnetic tracking are used. Other sensors such as optical sensors, acceleration sensors, or gyroscopes may be used to provide 5 DOF information of 6 DOF information.

[0090] For example, an optical tracking system using reflecting (passive) markers or active markers that can be detected in 3-D space as shown in FIG. 13, may be used. The combination of three markers with constant distance to each other ( 3 positions in 3-D space) allows acquiring $6 \mathrm{DOF}$, two markers with constant distance to each other allows acquiring $5 \mathrm{IOOI}$ respectively.

[0091] Images may he used which are captured from one or mere cameras calibrated to provide overlapping projections to compute the position (coordinates) of the markers in space. The markers are positioned so as to guarantee a sufficient difference in comparison to the rest of the caplured image to find their positions using video filtering techniques.

[0092] However, the use of magnetic sensors as in the first to third embodiments is more advantageous in that in case of the optical tracking system a so-called line of sight is needed. [0093] More than two types of sensors may be used (e.g. an acceleration sensor is used as the third sensor providing at least yaw and pitch information while sensor coils are used as the first and second sensors).

[0094] In the first embodiment, the distance between the distal interphalangeal joint $\mathrm{C}$ and the first sensor is defined, as lu with respect to a surface direction and $\mathrm{r}_{F}$ with respect to a radius direction, so that the joints $\mathrm{A}, \mathrm{B}$, $\mathrm{C}$, is positioned on an rotation axis of the finger. Although placing the joints on the finger axis allows a more accurate modelling of the position and orientation of the joints $\mathrm{A}, \mathrm{B}, \mathrm{C}$, it may be possible to define the distanee between the distal interphalangeal joint $\mathrm{C}$ and the first sensor in a different way (e.g. three joints or joint points are assumed to be not on the finger axis but on the surface of the finger).

[0095] The same can be said of the elbow joint Je and the wrist joint Jw in the third embodiment. That is, the elbow and wrist joints may be defined in a plane, which does not contain 
the forearm axis (although placing the two joints on the forearm axis allows a more accurate modeling of the arm).

[0096] In the first embodiment, the distance between the point on the dorsum and the metacarpal-phalangeal joint is measured. However, the following method may be used to evaluate the position of the metacarpal-phalangeal joint so that the measurement of the above-mentioned distance can be omitted (which may otherwise take place every time when the second sensor is attached).

[0097] Before the hand motion modeling is started, the x,y and z-position of the metacarpal-phalangeal joints will be found. For this purpose, the user will place his/her hand on a flat surface, such as table, within a measurement volume (c.g., of the magnetic field generator) with all fingers stretched. When the fingers are stretched the angle between the finger joints are assumed to be zero.

[0098] In the coordinate system $C_{s}$ of the first sensors the position of the metacarpal-phalangeal joints is:

$$
A_{s_{i}}=\left[0,0,0-\left(l u+1 \vec{b}_{i} l+l \overrightarrow{a b} i\right]\right.
$$

[0099] As such, the position of the metacarpal-phalangeal joint $\Lambda$ is then transformed to the local coordinate system of the first sensor in the same manner as for point $\mathrm{U}_{i}$ and $\mathrm{W}_{i}$. Further, the joint position is converted to the global coordinate system (i.e. the position and orientation of the first and second sensors are detected) and further to the local coordinate system of the second sensor $S_{6}$. After that, the hand motion modelling is started.

[0100] As a result, the position of the metacarpal-phalangeal joint $A$ relative to the second sensor $S_{6}$ can be oblained. Therefore, after the hand motion modelling is started, it is possible to follow the position of the metacarpalphalangeal joint A.

\section{INDUSTRIAL APPLICABILITY}

[0101] The present invention can be applied to a wide range of fields in which modelling of a hand/arm is needed.

[0102] lor example, information obtained from the hand is used to control a robotic hand. 'That is, a hand motion is tracked and translated into control signals to move the robotic hand.

[0103] Sensors or a glove with sensors may be altached to a non-human primate for a training purpose or a study on how the brain is encoding and planning grasp movements.

[0104] Furthermore, the modelling method and system may be used for a hand/arm animation in a film industry or in computer games.

1. $\Lambda$ method for modelling a position and orientation of a hand, comprising the steps of:

attaching at least a first sensor on a phalanx distalis of a finger, wherein said first sensor is adapted to provide information on at least five degrees of freedom that correspond to three translations, yaw and pitch;

placing a second sensor at a fixed position relative to a dorsum or palm of the hand, wherein said second sensor is adapted to provide information on at least six degrees of freedom that correspond to three translations, yaw, pitch and roll with respect to a point (Se) of the dorsum or paln of the hand;

detecting a position and orientation of each of the first and second sensors;

measuring a first distance between said point (S6) and a metacarpal-phalangeal joint (A) of the finger, a second distance between the metacarpal-phalangeal joint (A) and a proximal interphalangeal joint (B), a third distance between the proximal interphalangeal joint $(\mathrm{B})$ and a distal interphalangeal joint (C), and a fourth distance between the distal interphalangeal joint $(\mathrm{C})$ and the first sensor; and

calculating a position and orientation of each of the three joints on the basis of the measured first to fourth distances, the detected position and orientation of the first sensor, and the detected position and orientation of the second sensor.

2. A method according to claim 1 , wherein the first sensor comprises a sensor coil with its axis being placed along a longitudinal direction of the finger, and the second sensor comprises two sensor coils with their axes being placed in a geometrically defined position to each other so that the seeond sensor provides information on a roll of said point (S6), further comprising the step of:

generating a varying magnetic field so that the first and second sensors induce vollages, so that at the delection step the position and orientation of each of the first and second sensors is detected based on the induced voltages.

3. A method according to claim 1 , wherein the second sensor is arranged on the dorsum or palm of the hand.

4. A method of providing a signal to a processing device, comprising the steps of modelling a position and orientation according to one of the preceding claims and further comprising the step of generating at least one signal according to the calculated position and orientation.

5. A system for modelling a position and orientation of a hand, comprising:

a glove with glove fingers, comprising

at least a first sensor on a portion of the glove finger corresponding to a phalanx distalis of a finger, wherein said first sensor is adapted to provide information on at least five degrees of freedom that correspond to three translations, yaw and pitch; and

a second sensor at a position that is fixed on a portion of the glove finger corresponding to a dorsum or palm of the hand, wherein said second sensor is adapted to provide information on at least six degrees of freedom that correspond to three translations, yaw, pitch and roll with respect to a point of the dorsum or palm of the hand, and

a device comprising

a detector for detecting a position and orientation of each of the first and second sensors;

a storage for storing a first distane between said point and a first joint of the glove finger corresponding to a metacarpal-phalangeal joint of the finger, a second distance between the first joint and a second joint of the glove finger corresponding to a proximal interphalangeal joint of the finger, a third distance between the second joint and a third joint of the glove finger corresponding to a distal interphalangeal joint of the finger, and a fourth distance between the third joint and the first sensor; and

a calculator for calculating a position and orientation of each of the three joints on the basis of the stored first to fourth distances, the detected position and orientation of the first sensor, and the detected position and orientation of the second sensor.

6. $\Lambda$ system according to claim 5 , wherein the first sensor comprises a sensor coil with its axis being placed along a 
longitudinal direction of the glove finger, and the second sensor comprises two sensor coils with their axes being placed in a geometrically defined position to each other so that the second sensor provides information on a roll of said point, further comprising:

a generator for generating a varying magnetic field so that the first and second sensors induce voltages, thereby allowing the detector to detect the position and orientation of each of the first and second sensors based on the induced voltages.

7. A system according to claim 5, further comprising a generator for generating at least one signal according to the calculated position and orientation.

8. A system according to claim 5, further comprising a wireless transmitling device for transmilting an output of the first sensor and/or the second sensor to a receiver.

9. A method for modelling a position and orientation of a hand, comprising the steps of:

attaching at least a first sensor on a phalanx distalis of a finger, wherein said first sensor is adapted to provide information on at least five degrees of freedom that correspond to three translations, yaw and pitch;

placing a second sensor at a fixed position relative to a dorsum or palm of the hand, wherein said second sensor is adapted to provide information on at least six degrees of freedom that correspond to three translations, yaw, pitch and roll with respect to a point (S6) of the dorsum or palm of the hand;

measuring a first distance between a melacarpal-phalangeal joint (A) and a proximal interphalangeal joint (B), a second distance between the proximal interphalangeal joint (B) and a distal interphalangeal joint (C), and a third distance between the distal interphalangeal joint $(\mathrm{C})$ and the first sensor;

calculating a position of a metacarpal-phalangeal joint (A) relative to the first sensor in a state that the finger is stretched, on the basis of the first to third distances;

detecting a position and orientation of each of the first and second sensors; and

calculating a position and orientation of each of the three joints on the basis of the measured first to third distances, the calculated, relative position of the melacarpal-phalangeal joint (A), the detected position and orientation of the first sensor, and the detected position and orientation of the second sensor.

10. $\Lambda$ method according to claim 1 , further comprising steps of modelling a position and orientation of an arm, said steps comprising: attaching a third sensor (S7) on a forearm, wherein the third sensor is adapted to provide information on at least two degrees of freedom that correspond to yaw and pitch;

providing a position of a shoulder joint (Js);

detecting an orientation of the third sensor (S7) on the basis of an output from the third sensor (S7) and delected roll information of the second sensor (S6);

obtaining an orientation of a wrist joint ( Jw) and an elbow joint (Je) on the basis of the detected orientation of the third sensor (S7);

measuring a fifth distance between the wrist joint (JW) and the second sensor (S6);

calculating a position of the wrist joint (Jw) on the basis of the measured fifth distance and the detected position of the second sensor (S6);

measuring a length of the forearm; and

calculating a position of the elbow joint (Je) on the basis of the measured length of the forearm, the detected orientation of the third sensor (S7) and the calculated position of the wrist joint (Jw)

11. A system according to claim 5 , further adapted to model a position and orientation of an arm, wherein

the glove further comprises a third sensor (S7) on a glove portion corresponding to a forearm of the arm, wherein the third sensor is adapted to provide information on at least two degrees of freedom that correspond to yaw and pitch,

said detector is adapted to detect yaw and pitch information of the third sensor (S7);

said calculator is adapted to calculate an orientation of the third sensor (S7) on the basis of the detected yaw and pitch information of the third sensor (S7) and detected roll information of the second sensor (S6);

said storage is adapted to store a position of a shoulder joint (Js), a length of the forearm and a fifth distance between a wrist joint ( $\mathrm{Jw}$ ) and the second sensor (S6);

said calculator is adapted to obtain an orientation of the wrist joint (Iw) and an elbow joint (Je) on the basis of the detected orientation of the third sensor (S7), to calculate a position of the wrist joint (Jw) on the basis of the stored fifth distance and the detected position of the second sensor (S6), and to calculate a position of the elbow joint (Je) on the basis of the stored length of the forearm, the detecled orientation of the third sensor (S7) and the calculated position of the wrist joint (Jw). 




\subsection{Musculoskeletal representation of a large repertoire of hand grasping actions in primates}

In this chapter, a 3D-musculoskeletal model of the primate upper extremity is presented. For the first time, reach-to-grasp movements could be described entirely in the joint angle and the muscle domain in primates. Interestingly, simulations based on recorded hand kinematics revealed that the movements of the hand and arm could be expressed more compactly and precisely in the muscle domain than in the joint angle domain. The findings emphasize the intelligent design of the biomechanical system that is capable of controlling the many muscles of the upper extremity in a synergistic and in low-dimensional fashion. The chapter is included as published in IEEE Transactions on Neural Systems and Rehabilitation Engineering.

Follow links to access:

Supplemental video 1

Supplemental video 2 


\section{Musculoskeletal representation of a large repertoire of hand grasping actions in primates}

Musculoskeletal grasp movement modelling

Schaffelhofer S. ${ }^{\dagger 1}$, Sartori M. ${ }^{+2}$, Scherberger $\mathrm{H}^{* 1}$, and Farina, D. ${ }^{* 2}$

† equal first author, * corresponding author

${ }^{1}$ Department of Neurobiology at the German Primate Center GmbH, Kellnerweg 4, 37077 Göttingen, Germany.

${ }^{2}$ Neurorehabilitation Engineering, Universitätsmedizin Göttingen, 37077 Göttingen, Germany (email: d.farina@bccn.uni-goettingen.de

Acknowledgements: The authors thank R. Ahlert, N. Nazarenus, and L. Burchardt for assistance in animal training, M. Dörge for technical assistance, L. Schaffelhofer for glove production, and J. Michaels for comments on an earlier version of the manuscript. This work was supported by the BMBF (BCCN II, DPZ 01GQ1005C) and the European Research Council (ERC), Advanced Research Grant DEMOVE ("Decoding the Neural Code of Human Movements for a new Generation of ManMachine Interfaces"; contract \#267888). 


\section{Abstract}

Reach-to-grasp tasks have become popular paradigms for exploring the neural origin of hand and arm movement. This is typically investigated by correlating limb kinematic with electrophysiological signals from intracortical recordings. However, it has never been investigated whether reach and grasp movements could be well expressed in the muscle domain and whether this could bring improvements with respect to current joint domain-based task representations. In this study, we trained two macaque monkeys to grasp 50 different objects, which resulted in a high variability of hand configurations. A generic musculoskeletal model of the human upper extremity was scaled and morphed to match the specific anatomy of each individual animal. The primate-specific model was used to perform three-dimensional reach-to-grasp simulations driven by experimental upper limb kinematics derived from electromagnetic sensors. Simulations enabled extracting joint angles from 27 degrees of freedom and the instantaneous length of 50 musculotendon units. Results demonstrated both a more compact representation and a higher decoding capacity of grasping tasks when movements where expressed in the muscle kinematics domain than in the joint kinematics domain. Accessing musculoskeletal variables might improve our understanding of cortical handgrasping areas coding, with implications in the development of prosthetics hands.

Keywords: arm, grasping, hand, musculoskeletal model, non-human primates, upper extremity 


\section{Introduction}

Evolution in primates has led to an organization of large sets of muscles around multiple joints enabling complex movements in the upper extremity [1]. Reach-to-grasp tasks have become a popular paradigm for studying the mechanisms underlying movement generation in the nervous and musculoskeletal systems [2-7]. Due to the high level of implantation complexity and ethical implications, invasive recordings of neuromuscular activity are rare in humans $[8,9]$. Non-human primates (NHP) (i.e. macaca mulatta in this study) became the preferred research model due to their strong analogies to humans at the nervous and motor systems levels [10].

During reach-to-grasp actions, the dynamics of the musculoskeletal system has been studied with a main focus on limb and joint kinematics variables, including: hand velocity, direction, and joint angular position [5, 11-13]. These studies demonstrated strong correlations between the motor/premotor cortex neural activity and such kinematics variables in NHPs. In this context, movement was recorded using optical or electro-magnetic methods, based on non-anatomical models of finger, hand and arm segments [5, 14-16]. Previously proposed methodologies have never taken into account the kinematic behaviour of the underlying musculotendon units (MTUs) spanning the hand and upper extremity joints during reach-to-grasp movements.

This study uses musculoskeletal modelling and simulation for deriving MTUs kinematic variables noninvasively from the intact primate. This allows generating information that could not be easily accessed by experimental tests alone. These include the MTU instantaneous length, contraction velocity, and moment arms developed around multiple degrees of freedom (DOFs) in the finger, hand, and arm segments [17-21]. Current musculoskeletal models of the macaque monkey upper extremity $[17,21]$ do not account for the large number of DOFs and MTUs in the primate hand and are not suitable for studying complex reach-to-grasp tasks. In this study, a comprehensive musculoskeletal model of the human arm and hand was used as basis for exploring the complex NHP musculoskeletal function [18-20, 22]. This choice is further supported by extensive studies consistently confirming strong anatomical similarities between human and monkey primates [23, 24]. Scaling and morphing methods were then used to adapt each anatomical segment and muscle in the generic human model to match the specific anatomy of each primate [25].

In this study we assess the potentials of the scaled, animal-specific model for studying a large repertoire of grasping tasks in two macaque monkeys. For this, we studied how well features extracted at the joint level and at the MTU level respectively, could represent large repertoires of grasping actions. Although in humans and NHPs, the number of MTUs is by far larger than the number of DOFs in the skeletal joints, the benefit associated with this high dimensionality is not yet fully explored. In this study we hypothesize that the information extracted at the MTU level can 
produce more compact representations of a large repertoire of hand grasping actions with an associated improvement in grasping decoding capabilities than it would be possible by extracting information at the joint level, as done traditionally in the literature. This would emphasize on the inherent non-linearity between the skeletal and muscular systems. This could play a crucial role for understanding the efficient neuromuscular mechanisms underlying the control of large biological structures (i.e. muscles), which enable complex dexterous hand movement.

\section{Methods}

\section{Experimental Task}

Two macaque monkeys (macaca mulatta; animal $\mathrm{M}$ and $\mathrm{Z}, 7 \mathrm{~kg}$ male and $11 \mathrm{~kg}$ female, respectively) were trained to grasp a wide range of different objects of equal weight while wearing an instrumented glove to track their hand and arm motion [see supplementary movie 1-2]. Animal care and all experimental procedures were conducted in accordance with German and European laws governing animal care and were in agreement with the Guidelines for the Care and Use of Mammals in Neuroscience and Behavioral Research [26].

(a)

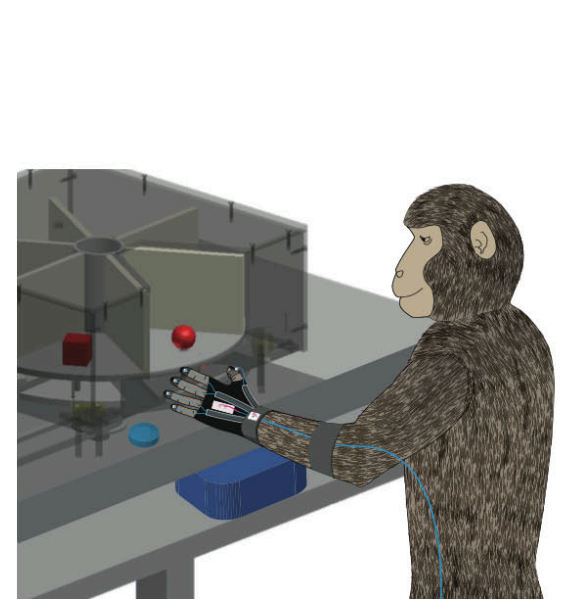

(b)

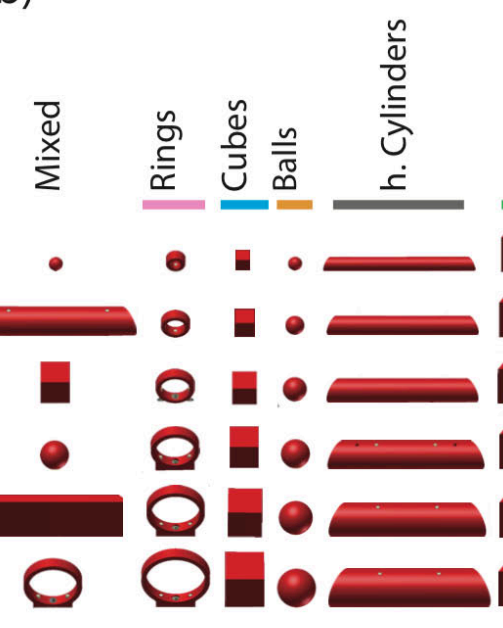

(c)

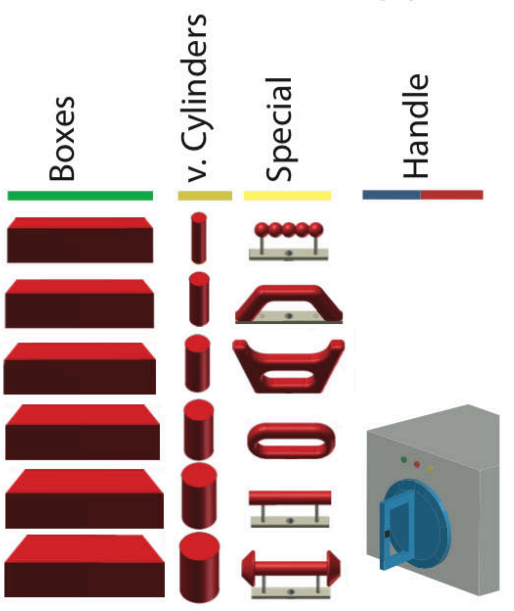

Fig. 1. Behavioural task. (a) Two rhesus monkeys were trained to grasp a wide range of objects presented on a PC-controlled turntable. During a recording session the animals wore an instrumented glove holding electromagnetic sensor coils for tracking finger, hand, and arm movements. The magnetic field generator was placed below the turntable (blue box). (b) In total, 48 objects of different shape and size were presented to the animals, including rings (pink), cubes (blue), balls (orange), horizontal cylinders (grey), boxes (green), vertical cylinders (beige) and special forms (yellow). (c) Additionally, the monkeys performed precision (red) and power grips (blue) on a handle.

Objects were presented to the monkeys on a PC-controlled turntable in front of them (Fig. 1(a)). In total, each animal grasped 48 objects of different shape and size (Fig. 1(b)) including spheres, rings, 
cubes, horizontal cylinders, boxes, vertical cylinders, and further six objects having more complex forms. The turntable could be replaced by a handle (Fig. 1(c)) that the animals grasped with a precision grip (touching the centre of the handle with index finger and thumb) or a power grip (enclosure of the handle using digits). This resulted in a total of 50 different grasping conditions, which the monkeys repeated for at least 10 times during each experimental session. All repetitions were performed in a controlled trial sequence in which the monkey had to release a switch at the resting position, grasp for and hold the randomly selected object or the handle for $0.5 \mathrm{~s}$.

All digital signals affecting and monitoring the animal's behaviour were controlled by a customwritten software implemented in Labview Realtime (National instruments; time accuracy: $1 \mathrm{~ms}$ ) and were recorded together with the hand and arm kinematics.

\section{Tracking of Hand and Arm Movements}

The musculoskeletal simulations presented in this study required comprehensive motion data describing finger, hand, and arm movements. To record this kinematics we used an instrumented glove equipped with seven electro-magnetic sensors (Fig. 1(a)) that was previously reported in detail $[14,27]$. In short, the method accessed the orientation and the position of seven electro magnetic sensors that were placed on top of the fingernails of digits and thumb, the hand's dorsum and the wrist of the hand. In this way the position of the fingertips ( ${ }^{f} \mathrm{TIP}$ ), distal interphalangeal joints ('DIP), metacarpal phalangeal joints ( ${ }^{f} \mathrm{MCP}$ ), and the wrist (W) could be derived from the sensors ( $f \in\{1,2, \ldots, 5\}$ ). Exploiting the manually measured segment length and the anatomical restrictions of the hand allowed computationally estimating the position of the proximal interphalangeal joints ('PIP), elbow (E), and shoulder (S) at high accuracies [14]. This gave a total of 18 joint positions in 3-D space (experimental markers).

\section{Pre-processing of Kinematics}

Additional to the 18 joint centers, two more markers ( $E^{\prime}$ and $\left.W^{\prime}\right)$ were defined to better constraint the simulation throughout the task and were defined as function of the existing joint positions. Whereas vectors $\overrightarrow{E W}$ and $\overrightarrow{S E}$ allowed describing the orientation of forearm and upper arm respectively, the additional two markers defined their rotation. The $E^{\prime}$ marker was placed orthogonal on the plane $P 1$, described by $\mathrm{W}, \mathrm{E}$, and $\mathrm{S}$ and was placed $d=20 \mathrm{~mm}$ distant from its origin $\mathrm{E}$ :

$$
E^{\prime}=E+d n 1
$$

where $\mathrm{n} 1$ was the unit vector normal to P1 pointing to the side of the medial epicondyle of the humerus (Fig. 2(b)). 
The marker $W^{\prime}$ was located orthogonal to the sagittal plane $(P 2)$ of the hand (described by the reference sensor at the back of the hand) $20 \mathrm{~mm}$ distant from its origin $\mathrm{W}$ :

$$
W^{\prime}=W+d n 2
$$

where $\mathrm{n} 2$ was the unit vector normal to $\mathrm{P} 2$ pointing to the radial side of the hand (Fig. 2(b)).

Markers $E^{\prime}, E$ and $S$ provided sufficient information to describe the rotation, elevation and adduction/abduction of the humerus at the shoulder when applied to the driven musculoskeletal model. The marker $W^{\prime}$ gave information about the pronation/supination of the hand, which was directly linked to the rotation of the lower arm, e.g., ulna and radius. The 3-D trajectories of the 25 experimental markers (18 joints, five fingertips and two additional help points) were transformed into the coordinate system of the musculoskeletal model and taken as its input. We refer to them as the experimental markers driving the model (Fig. 2(a)).

All kinematic data were recorded at a sampling rate of $100 \mathrm{~Hz}$. Three-dimensional coordinates of each experimental marker were then exported to our musculoskeletal model realized in OpenSim, a software system allowing users to develop models of musculoskeletal structures and to create dynamic simulations of movement [25].

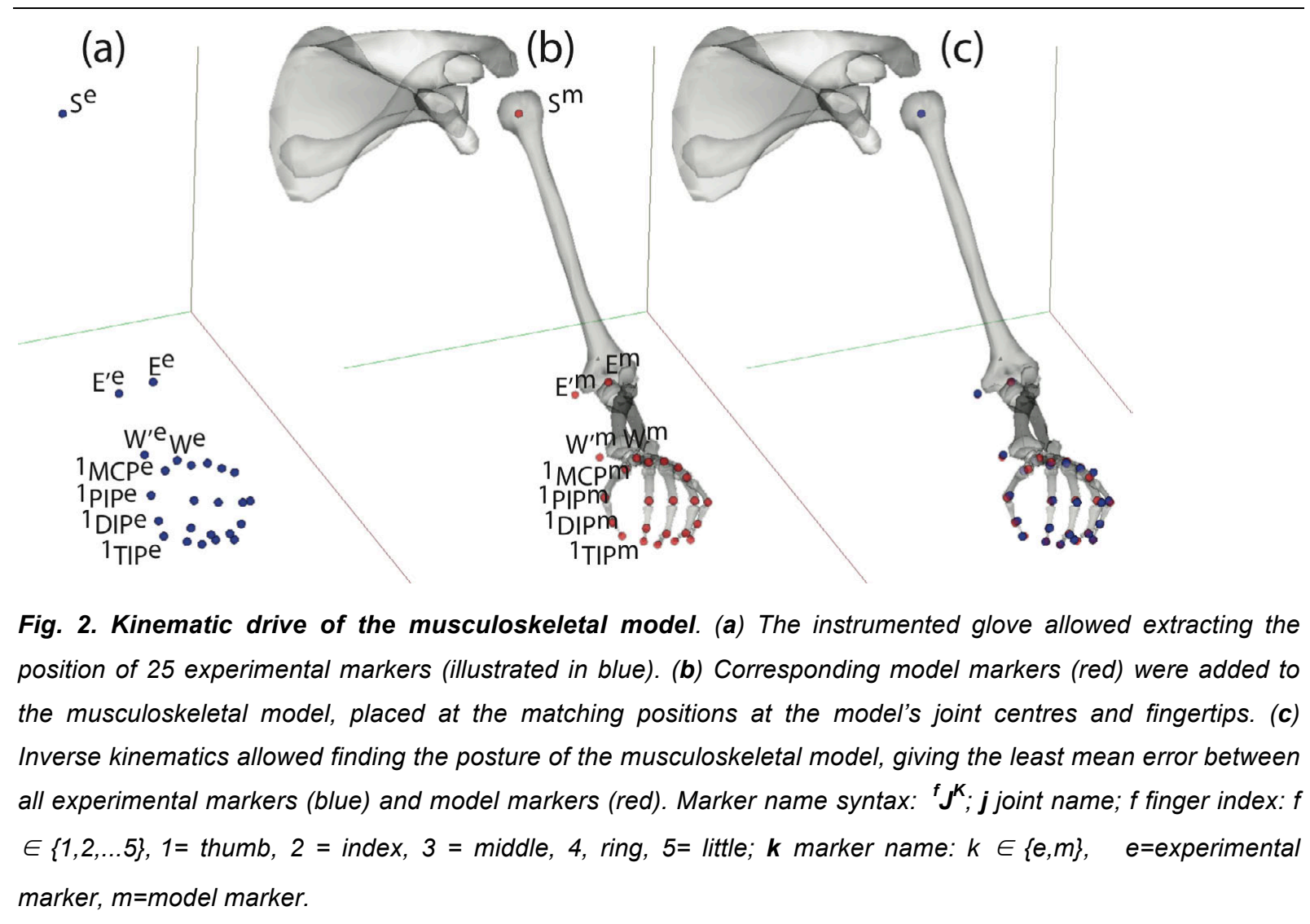




\section{Musculoskeletal Model}

\section{Model Description}

The musculoskeletal model employed in this study was adapted from an anatomically correct musculoskeletal model of the human upper extremity. This incorporated a total of 15 DOFs in the joints of the upper extremity, thumb and index segments (Table I) [18]. For this study, we extended the original musculoskeletal model to include four additional DOFs in each of the remaining fingers (middle, ring, little) as reported in Table I. In this, the axes of rotation of the joints in each finger were determined as the long axis of cylinders fit to the articular surfaces of the metacarpal and phalangeal bones as previously described [18]. Our proposed extended model had a total of 27 DOF in the hand and arm segments.

The model incorporated a total of 50 MTUs [18]. Each MTU in the model was defined by a set of points and surfaces attached to the underlying anatomical segments. The MTU attachments were determined from digitized muscle insertions and anatomical description [18]. This allowed generating anatomically accurate MTU paths over the range of motion of the considered 27 DOFs. The path of each MTU corresponded to the instantaneous MTU length $\left(e^{\mathrm{MTU}}\right)$ measured from origin to insertion and accounting for intermediate MTU-to-bone wrapping points and surfaces. All included MTUs are listed in [18].

TABLE I

JOINT AND DEGREES OF FREEDOM INCLUDED IN THE MUSCULOSKELETAL MODEL

\begin{tabular}{|c|c|c|}
\hline Joint & Degrees of Freedom (DOFs) & DOF Acronym \\
\hline Shoulder & elevation, rotation, and adduction/abduction & shELE, shROT, and shABD \\
\hline Elbow & flexion/extension & elbFE \\
\hline Wrist & flexion/extension, deviation, and pronation/supination & wristFE, wristDEV, and wristPS \\
\hline Thumb (I) Carpometacarpal & adduction/abduction and flexion/extension & $\mathrm{cmcABD}$ and $\mathrm{cmcFE}$ \\
\hline Thumb (I) Metacarpophalangeal & flexion/extension & mcpFE \\
\hline Thumb (I) Interphalangeal & flexion/extension & ipFE \\
\hline Fingers (II-V) Metacarpophalangeal & adduction/abduction and flexion/extension & mcpABD \\
\hline Fingers (II-V) Proximal Interphalangeal & flexion/extension & pipFE \\
\hline Fingers (II-V) Distal Interphalangeal & flexion/extension & dipFE \\
\hline
\end{tabular}

The symbol (II-V) refers to the index (II), middle (i.e. III), ring (IV), and little (V) fingers. The thumb finger is referred to as the first finger (I).

\section{Model Adaptation}

For accurate simulations, we scaled each skeletal segment and MTU in the generic model to match the primate specific anatomy for each specific animal using the 25 experimental marker positions (Fig.2(a)) tracked by the instrumented glove. The tracking technique explores the manually measured segment length, anatomical constraints of hand and arm, and the accurate spatial and rotational information of 7 sensor coils (see chapter 2.5 in [14] for detailed information). As described previously, measured and computationally derived experimental markers provided high accuracies [14] and were therefore selected for scaling. 
The model scaling procedure was performed using the OpenSim software package ${ }^{1}$ [25]. A set of model markers (Fig 2(b)) was placed in the generic human musculoskeletal model in correspondence to the experimental marker positions extracted from the primate [25]. The dimensions of each anatomical segment in the generic musculoskeletal model were then adjusted to minimize the relative distance between each pair of experimentally recorded marker locations and the corresponding model marker locations (see Fig 2(c) for a match between model and experimental markers). The MTU's insertion, origin, and MTU-to-bone wrapping points were adjusted proportionally so that they remained in the same relative position within the anatomical segment they were attached on [25]. This allowed adjusting the dimensions of each anatomical segment as well as the MTU paths in the generic human musculoskeletal model to the actual primates' anatomy.

\section{Inverse Kinematics and Extracted Features}

The scaled musculoskeletal model was then used to calculate angles and length (i.e. $\ell^{\mathrm{MTU}}$ ) in all DOFs and MTUs respectively. An inverse kinematics (IK) problem [25] was solved in OpenSim to determine the model DOF angles that best reproduced the experimentally recorded marker trajectories obtained from the magnetic glove system. As a result, for each frame in the simulation DOF angle values were computed, which positioned the scaled model in a pose that best matched the experimentally recorded marker trajectories (Fig. 2(c)). As the simulated model tracked the experimental marker trajectories frame-by-frame over time, instantaneous estimates of DOFs and $e^{\mathrm{MTU}}$ were calculated and stored. The simulated data were resampled at $50 \mathrm{~Hz}$ and low-pass filtered by a Kaiser window finite impulse response filter (pass-band cut-off: $2 \mathrm{~Hz}$, stop-band cu-toff: $8 \mathrm{~Hz}$, pass-band ripple: $1 \%$, stop-band attenuation: $20 \mathrm{~dB}$ ), as suggested previously [11]. The $27 \mathrm{DOFs}$ and $50 \mathrm{e}^{\mathrm{MTU}}$ s represent the features used in this study for the subsequent analysis of the grasping tasks.

\section{Analysis}

\section{Model Fit}

This first test assessed how well the scaled model reproduced the actual kinematics recorded experimentally from each primate upper extremity. For this, the root mean squared error (RMSE) between experimental (Fig. 2(a)) and model markers (Fig. 2(b)) was calculated to quantify the accuracy of both matching the primate anatomy and tracking the upper extremity kinematics:

$$
R M S E=\sqrt{\frac{1}{N} \sum_{n-1}^{N}\left\|x(n)^{\text {experimental }}-x(n)^{\text {model }}\right\|^{2}}
$$

\footnotetext{
${ }^{1}$ OpenSim release 2.4.0 available from: https://simtk.org/home/opensim
} 
where $x^{\text {experimental }}$ and $x^{\text {model }}$ represented the three-dimensional position of a specific marker as placed on the actual primate (i.e. experimental marker) and on the associated scaled model (i.e. model marker) respectively. The $N$ term is the total number of time frames in each simulated trial. This allowed quantifying the experimental kinematics tracking accuracy within each trial.

\section{Computation Speed}

In this test the IK computation time was examined. This was calculated as the average time needed to extract one frame of DOF and MTU kinematics from all joints and muscles in the model. The time needed to solve the IK problem from all trials and animals was averaged with respect to the total number of frames. Tests were performed on an 8 GB RAM, Intel Core i7 CPU @ $2.93 \mathrm{GHz}$ machine.

\section{Principal Component Analysis of Muscle and Joint Angle Patterns}

The extracted DOFs and MTU kinematics exhibit a large number of variables, making them difficult to interpret and visualize. However, multidimensional datasets often show redundancies among their variables. Principal component analysis (PCA) makes use of these correlations and allows for simplification of multidimensional hand variables [28] by creating a new set of features (principal components) that are uncorrelated and orthogonal to each other.

Animals were trained to grasp and hold each object for half a second. This epoch was well suited for PCA, since it showed the greatest variability of hand kinematics across conditions. We therefore examined the DOF and the $e^{\mathrm{MTU}}$ in the holding epoch for all correctly performed trials. Before computing the principal components, both measures were standardized to zero mean and unit standard deviation (Matlab function: zscore), which eliminates the influence of different measurement scales. The PCA analysis was performed over all correctly performed trials, which resulted in at least 500 trials in the DOF- and MTU space.

For comparing both spaces, we computed the Euclidean distance (ED) between all pairs of correctly performed trials within their complete multidimensional representations (Matlab function: $p d i s t$ ) and then correlated the resulting distance matrices for both spaces (Pearson correlation coefficient, Matlab function: corrcoef).

Additionally, cluster quality was expressed in both representations as the Dunn Index (D) aiming to identify dense and well-separated clusters [29]. The index is defined as the ratio between the minimal inter-cluster distance to maximal intra-cluster distance:

$$
D=\min \left\{\frac{d\left(C_{i}, C_{j}\right)}{\Delta_{\max }} \mid 1 \leq i<j \leq K\right\}
$$

where $d\left(C_{i}, C_{j}\right)$ represents the distance between clusters $i$ and $j$ and $\Delta_{\max }$ the maximum intercluster distance from all $K$ clusters. 


\section{Object Classification Accuracy}

The large repertoire of objects did not only cause a rich diversity of grip types, but also contained intended redundancies (different objects that were grasped in a similar fashion). These similarities among the different objects were used to evaluate and compare the information content carried in the MTU and the DOF space. As a measure, we determined how accurately the grasped objects could be decoded from the extracted DOF angles or, alternatively, from the $e^{\mathrm{MTU}}$. For this purpose, we implemented a likelihood decoder based on the Bayesian theorem [30]:

$$
p\left(c \mid k_{i}\right)=\frac{p\left(k_{i} \mid c\right) \cdot p(c)}{p\left(k_{i}\right)}
$$

In a first step, the decoder was trained with a segment of the recorded data to acquire $p\left(k_{i} / c\right)$, which is the probability of observing the kinematic variable $k$ ( $e^{\text {MTU }}$ or DOF) on channel $i$ in condition $c$. Probability distributions were modelled using Poisson distributions. In total, 50 different grasping conditions were decoded (48 objects plus precision and power grip in the handle task). In the DOF and the MTU space, 27 and 50 channels (number of DOF or MTU) were available, respectively. Since $p(c)$ is uniformly distributed and $p\left(k_{i}\right)$ does not depend on condition $c$, both terms can be summarized by a normalization factor $n_{i}$ that is independent of condition $c$. This allows expressing the probability of $c$ for given $k_{i}$ as:

$$
p\left(c \mid k_{i}\right)=n_{i} \cdot p\left(k_{i} \mid c\right)
$$

In a second step (decoding), a different segment of the recording was used to find the most likely condition for the observed kinematic variable $k$. To predict the decoded object, the likelihood function

$$
L(c)=\prod_{i=1}^{N} p\left(c \mid k_{i}\right)
$$

was evaluated for all conditions $\mathrm{c}$ and the condition $\widehat{c}$ selected as the predictor that maximized this function: $\hat{c}=\operatorname{argmax}_{c}(L(c))$. Since the factor $n_{i}$ in equation (5) was constant across all conditions, it could be ignored for the maximization and the calculation reduced to:

$$
\hat{c}=\operatorname{argmax}_{c}\left(\prod_{i=1}^{N} p\left(k_{i}|c|\right)\right) .
$$

Leave-one-out cross validation ensured that data sets for training were not used for testing.

For comparing the classification accuracy based on joint-angle or muscle patterns, we computed the decoding accuracy vs. the number of included objects $(n=\{1, \ldots, 50\})$. To rank the objects according to their separability, we first extracted the kinematic variables $\left(e^{\mathrm{MTU}}\right.$, DOF) from the mean hold epoch and computed the Mahalanobis distance (MD) between each pair of objects groups (Matlab function: manova1). The two objects (groups) showing the largest MD to each other (most separated in $\mathrm{e}^{\mathrm{MTU}}$ / DOF space) were added first to the decoder. Next, a new decoder was built by adding the object that maximized the performance in combination with previously selected objects. 
This "greedy" selection process was continued by gradually adding one more object at a time and rebuilding the decoder [5]. Using this procedure allowed to rank the objects based on their separability in the DOF and MTU space. Objects that could be separated best by the decoder were ranked first.

\section{Results}

The model was successfully used to provide fast, accurate, and reliable musculoskeletal descriptions for a large repertoire of grasping movements in primates.

\section{Extracted Parameters}

All extracted model parameters allowed visualizing the course of motion continuously over time. As an example, we present the kinematic and muscular variables of three subsequent grasping movements together in (Fig. 3) and as supplementary videos [see video 1 for DOF extraction and video 2 for MTU extraction].

The parameters clearly demonstrated a distinct approach for grasping the three objects: ring, sphere, and cylinder (i.e. Fig. $3 \mathrm{~b}-\mathrm{c}$ at times $t 1, t 2$, and $t 3$ respectively). The ring was grasped using power grips, requiring a strong rotation (supination) of the wrist of about $-70^{\circ}$ (i.e. see Wrist DOF variable in Fig. $3 \mathrm{~b}$ at time 1 ). In macaque monkeys, the rotation of ulnar and radius is mainly achieved by the pronator teres (PT) and the pronator quadratus muscle (PQ, i.e. see Wrist $\ell^{M T U}$ variable in Fig. $3 \mathrm{c}$ at time $t 1$ ) for pronating the forearm, whereas the supinator (SUP, i.e. see Wrist $\ell^{M T U}$ variable in Fig. 3c at time t1) and the biceps muscles (BIClong, BICshort; long and short head respectively) are responsible for supination. In addition, the brachioradialis muscle (BRD) moves the forearm back to a neutral position, either from a supination or from a pronation posture. Results show how grasping the ring led to a supination of the forearm with a shortening of the SUP-muscle and a simultaneous lengthening of the PQ-muscle (i.e. see Wrist $\ell^{M T U}$ variable in Fig. $3 \mathrm{c}$ at time $t 1$ ). When the monkey grasped the sphere or the cylinder, the rotation of the forearm was minimal, therefore affecting the corresponding muscles (i.e. PQ and SUP) less (i.e. see Wrist $\ell^{M T U}$ variable in Fig. $3 c$ at time $t 2$ and $t 3$ ). Grasping the small sphere required a more precise control of index finger and thumb with longer grasping time needed in comparison to ring and cylinder. Furthermore, this grip type caused a strong flexion of index finger and thumb, which was also reflected in the corresponding length of thumb and index finger muscles, including the flexor pollicis longus (FPL), the extensor pollicis longus (EPL) as well as the flexor digitorum profundus index (FDPI) and the extensor indicis proprius (EIP) (i.e. see Thumb $\ell^{M T U}$ and Index $\ell^{M T U}$ variables in Fig. $3 c$ at time $t 2$ ). In comparison to the other two objects, the cylinder was grasped with a whole-hand grip achieved by digits and thumb requiring less flexion 88 
of the fingers that caused less shortening of the muscles (i.e. see Thumb $\ell^{\text {MTU }}$ and Index $e^{\text {MTU }}$ variables in Fig. $3 \mathrm{c}$ at time $t 3$ ).

These findings demonstrate the ability of our musculoskeletal model to extract joint angle- (DOF) and muscle kinematics $\left(e^{\mathrm{MTU}}\right)$ for describing finger, hand, and arm movements.

(a)

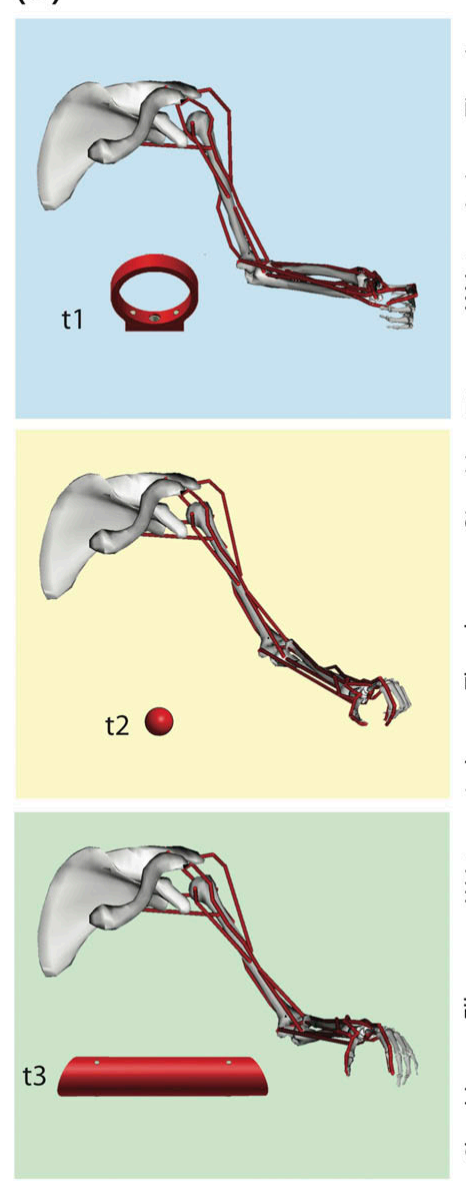

(b) $\mathrm{t}$ 1 t2 t3

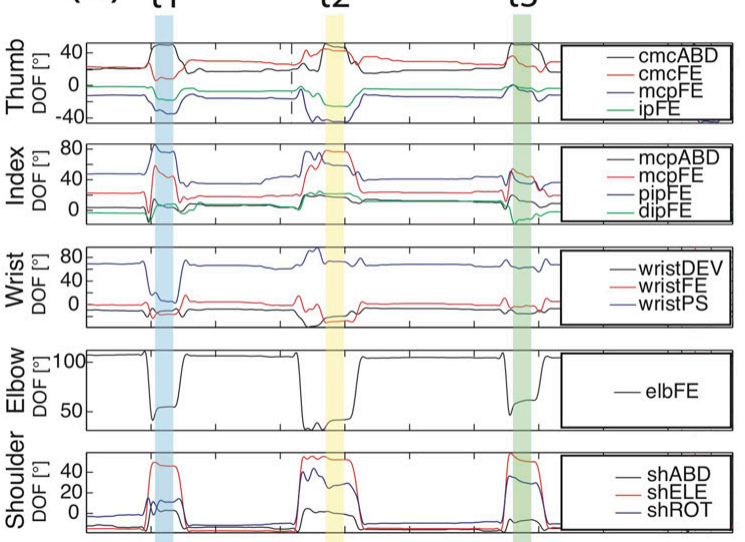

(c)
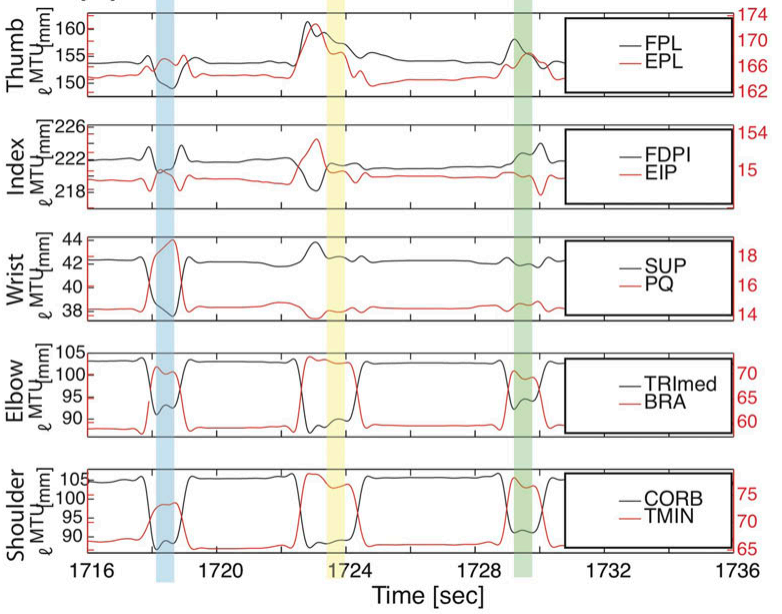

Fig. 3. Model features. The musculoskeletal model (a) allowed extracting joint angles (b) as well as muscle kinematics (c). In total, the information of 27 DOFs (18 joints) and $50 l^{M T U s}$ could be accessed. (a) Hand and arm posture of the monkey while grasping three different objects in the hold period of sample trial t1 (ring), t2 (ball), and $t 3$ (cylinder). In addition to the skeletal configuration, a selection of MTUs is illustrated for these trials in red [anterior deltoid (antDELT), posterior deltoid (postDELT), teres minor (TMIN), teres major (TMAJ), coracobrachialis (CORB), triceps long head (TRllong), biceps long head (BIClong), brachialis muscle (BRA), extensor carpi radialis brevis (ECRB), flexor carpi radialis (FCR), FDPI (flexor digitorum profundus index), extensor indicis proprius (EIP), extensor pollicis longus (EPL), extensor pollicis brevis (EPB)]. (b) Example DOFs of the thumb, index finger, wrist, elbow, and shoulder while subsequently grasping three objects. (c) $\ell^{M T U}$ of example muscles linked to the joints in (b).

\section{Model Fit}

Results from the model fit test revealed an average RMSE error of $2.06 \pm 1.64 \mathrm{~mm}$ and $2.33 \pm 1.50 \mathrm{~mm}$ (mean \pm standard deviation) across all pairs of experimental and model markers from all trials 
performed by animal $Z$ and $M$ respectively. The marker RMSEs in animal $Z$ ranged from $0.77 \pm 0.13 \mathrm{~mm}$ (i.e., DIP marker of ring finger) to $5.66 \pm 0.31 \mathrm{~mm}$ (i.e., MCP marker of little finger). For animal $\mathrm{M}$, marker RMSEs ranged from $0.93 \pm 0.22 \mathrm{~mm}$ (i.e. DIP of middle finger) to $8.26 \pm 0.10 \mathrm{~mm}$ (i.e. MCP of thumb marker). Across all trials and animals, the majority of average RMSE errors between experimental and model markers were smaller than $3 \mathrm{~mm}$ (for 40 out of 49 pairs) as shown in Fig. 4 .

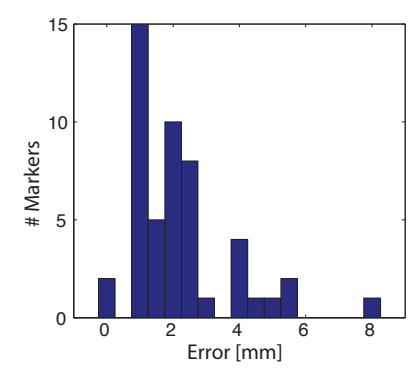

Fig. 4. Test of model fit accuracy. The histogram shows RMSEs (Eq. 3) derived from all pairs of experimental and model markers averaged across all trials and animals (50 markers in total).

Importantly, these RMSE values are small in comparison to objects sizes that ranged from $15 \mathrm{~mm}$ to $60 \mathrm{~mm}$ in diameter or length (balls and cubes respectively). Therefore, model-fit accuracies were well suited for investigating the musculoskeletal representations performed on the set of objects.

\section{Computation Speed}

The recording sessions from both animals produced extensive datasets that were used to assess the simulation speed of our musculoskeletal model. We found that performing inverse kinematics with OpenSim, despite providing a more complex musculoskeletal description of hand and arm, was significantly faster to compute than our previous, simpler kinematic model (KinemaTracks, [14]). The OpenSim's IK could process both sessions (i.e. 284795 and 342335 frames from animal Z and M, respectively) in 15:08- and 17:31 minutes whereas KinemaTracks required 18:12- and 19:55 minutes. In this, the average time needed to extract one frame of DOF and MTU kinematics from all joints and muscles required $3.1 \mathrm{~ms}$ of computation time. The musculoskeletal model therefore allowed extracting the motion data faster than real-time, and can be readily used to process extensive datasets such as the ones presented here.

\section{Principal Component Analysis of Muscle and Joint Angle Patterns}

We used PCA to interpret and visualize the large repertoire of grasping movements in both the DOF and $\ell^{\mathrm{MTU}}$ space. As an example we plotted the first two PCA components in the hold epoch of animal $Z$ for both, the DOF and $e^{\mathrm{MTU}}$ representation (Fig. 5(a) and Fig. 5(b) respectively). The plots 
demonstrate a high variability of joint angle and muscle patterns across the large number of objects and trials.

For animal $Z$, the $1^{\text {st }}$ PCA component roughly represented the aperture of the hand, pointing from the condition of smallest (precision grip) to the condition of highest aperture (big boxes), whereas the $2^{\text {nd }}$ component approximately reflected the monkey's wrist orientation. We also used PCA to reveal similarities and dissimilarities between grasping movements.

For example, smaller objects including the small ball, cube, or ring were grasped with a similar grip, using index finger and thumb, and were therefore located close to each other in PCA space. Even objects of largely diverse shape might be grasped very similarly such as the vertically aligned cylinders, big rings, and the handle. All of these objects were grasped with a kind of power grip (rotation of the forearm and enclosure with four digits).

Notably, the DOF and the $e^{\mathrm{MTU}}$ space showed a strong similarity within PCA coordinates. Almost the same cluster-overlaps were observed for the DOF and for the MTU representation (Fig. 5(a-b)). Computing the Pearson correlation coefficient between both multidimensional spaces further demonstrated the strong link between the DOF and the $e^{\mathrm{MTU}}$ representation (cc=0.85 and 0.75 for animal $Z$ and $M$ respectively). These similarities were expected, since the muscle patterns and the joint angle patterns are strongly linked to each other and both reflect the shape and size of the grasped objects.

Although, the representation of grasping movements was similar in the DOF and in the MTU space, how many linearly uncorrelated components were actually engaged in the task remained an open question. The large number of natural grip types and the resulting large variability in our dataset allowed us to answer this question. We determined how many of principal components were required to reproduce the individual grip types (Fig. 5(c)). We found that 8 PCA components were sufficient in both animals to explain more than $95 \%$ of variance across all conditions in the MTUspace, whereas in the DOF-space 11 (animal Z) and 12 components (animal M) were needed to explain the same amount of variance. The lower dimensional representation in the $e^{\mathrm{MTU}}$ space is remarkable, since the number of MTUs (i.e., 50) involved in grasping strongly exceeded the number of DOF (i.e., 27).

In addition to these findings, we tested whether the 50 object conditions were also represented more compactly in the $e^{\text {MTU }}$ than in the DOF space by computing the Dunn index (D)[29]. In both animals, the condition clusters were represented more separated when expressed in the $\ell^{\mathrm{MTU}}$ space than in the DOF space (D for animal Z: 1.17 DOF, 1.39 ( $\left.e^{\mathrm{MTU}}\right)$; animal M: $0.57 \mathrm{DOF}, 0.714 \mathrm{e}^{\mathrm{MTU}}$ ).

These findings emphasize the importance of the musculoskeletal organization for the control of hand movements, which might become highly relevant for the development of future hand prosthetics 
[31].
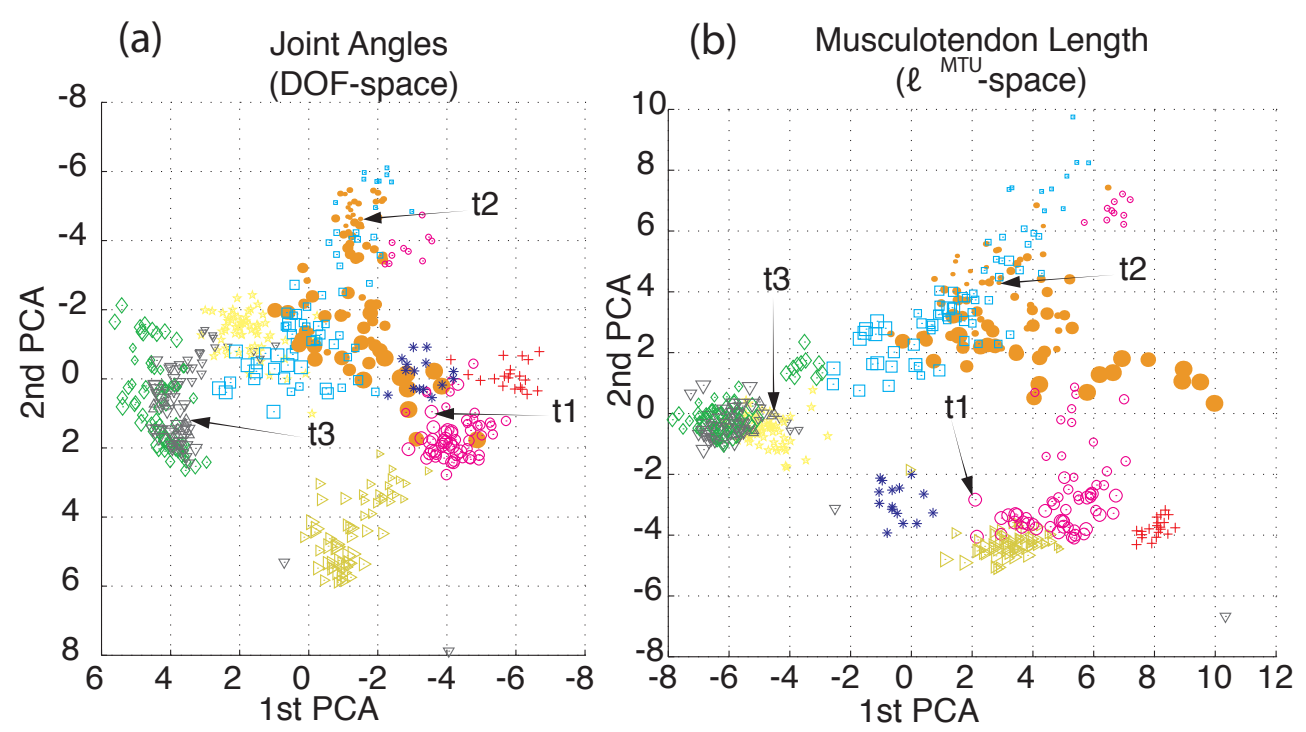

(c)

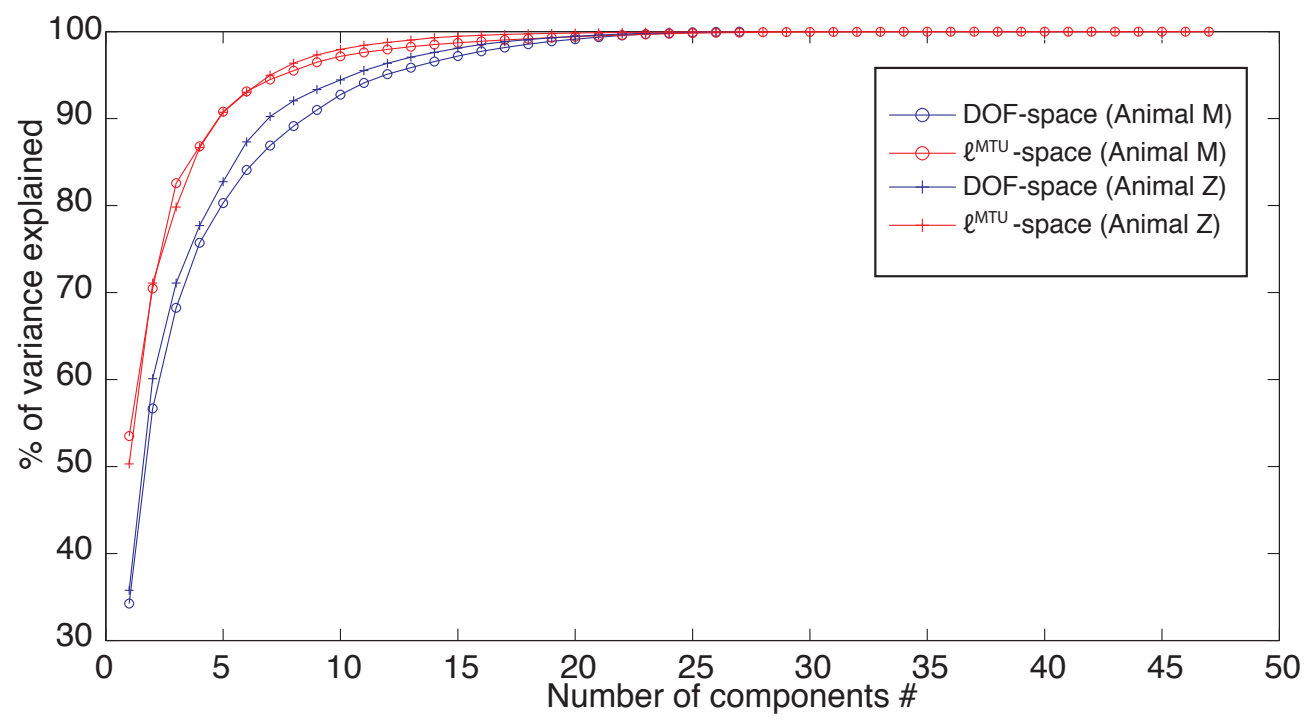

Fig. 5. Dimensionality estimation. The principal components were computed across all correctly performed trials in the space of all DOFs (a) as well as in the space of all $\ell^{\mathrm{MTU}}$ 's (b). Each symbol in (a) and (b) represents the DOF or $\ell^{\text {MTU }}$ state of a correctly performed trial while the monkey is holding an object. The colour of each symbol represents the shape of the object whereas the symbol size reflects the actual size of the object (colour code according to Fig. 1). The arrows in a-b point to the three selected grips presented in Fig. 3 at time t1, t2 and t3. (c) Percentage of variance explained as a function of the (sorted) number of principal components of DOFspace (blue) and $\ell^{M T U}$-space (red) for monkey $Z$ and M. As can be seen, the first eight PCA components are sufficient to explain $95 \%$ of the variance in the $\ell^{M T U}$-space. 


\section{Object Classification Accuracy}

As a measure of discriminability, we implemented a Bayesian classifier for decoding objects based on the extracted DOF or $\ell^{\mathrm{MTU}}$ parameters (27 and 50 dimensions, respectively).

Starting with a decoder of including only two objects, we iteratively added more objects to the decoder in a "greedy" fashion based on their separability (see Methods section). As shown in Fig. 6a (solid dots) the decoding accuracy remained stable until objects were included, that evoked similar DOF or $e^{\mathrm{MTU}}$ representations with respect to other objects. As a consequence, the decoding accuracies dropped. Importantly, the $\mathrm{e}^{\mathrm{MTU}}$-representation of grasping movements was better suited to distinguish individual objects than with the DOF representation. These findings were consistent in both animals and in agreement with the cluster analysis results. To test, whether this effect remained with an equal number of dimensions, we decoded the same objects with a randomly selected set of 10 dimensions (100 repetitions) in the $e^{\mathrm{MTU}}$ and DOF space. As shown in Fig. 6a (dashed lines), $e^{\mathrm{MTU}}$ still provided a better decoding in comparison to DOFs.

Further tests were performed on all dimensions. In the complete DOF space, the decoding accuracy was better than $95 \%$ for up to a maximum number of 20 objects in both animals. In comparison, the same decoding accuracy was achieved with a maximum number of 28 and 26 objects in the MTU space for animal $M$ and $Z$, respectively. Beyond these thresholds the decoding accuracy decreased linearly with increasing number of objects (i.e., less separable objects).

To quantitatively estimate the point of accuracy drop, we implemented the L-method [32]. The algorithm searches for the two piecewise linear regression lines that fit the decoding results best (solid lines, Fig.6a). For this, the algorithm gradually changes the intersection points of the two lines and measures their fit. The RMSE error produced by this fit is plotted in Fig. 6(b) for variable intersection points. Results highlight a better resolution and separability between objects when using the $e^{\mathrm{MTU}}$ description of grasping movements than rather than the DOF description.

Additionally, we computed the PCA components accounting for $95 \%$ of the variance of the data in the $e^{\mathrm{MTU}}$ and DOF space as a function of the number of included objects in the greedy selection. $6(\mathrm{c})$ shows a strong increase in variability when the most separable objects (ranked first) were added. However, the variability reached a plateau already for a small number objects in both animals. This demonstrated that including similar objects could not increase the variability of grip types further and explained the significant drop of decoding accuracy for larger object sets. Classifying the objects based on $e^{\mathrm{MTU}}$ and DOF demonstrated a higher separability and resolution in the MTU-space. 
(a)

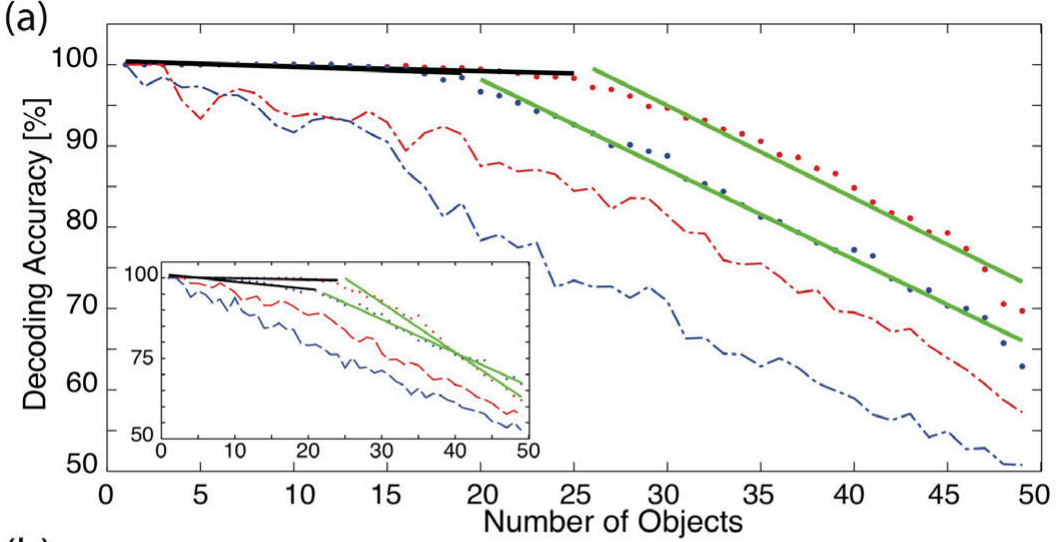

(b)
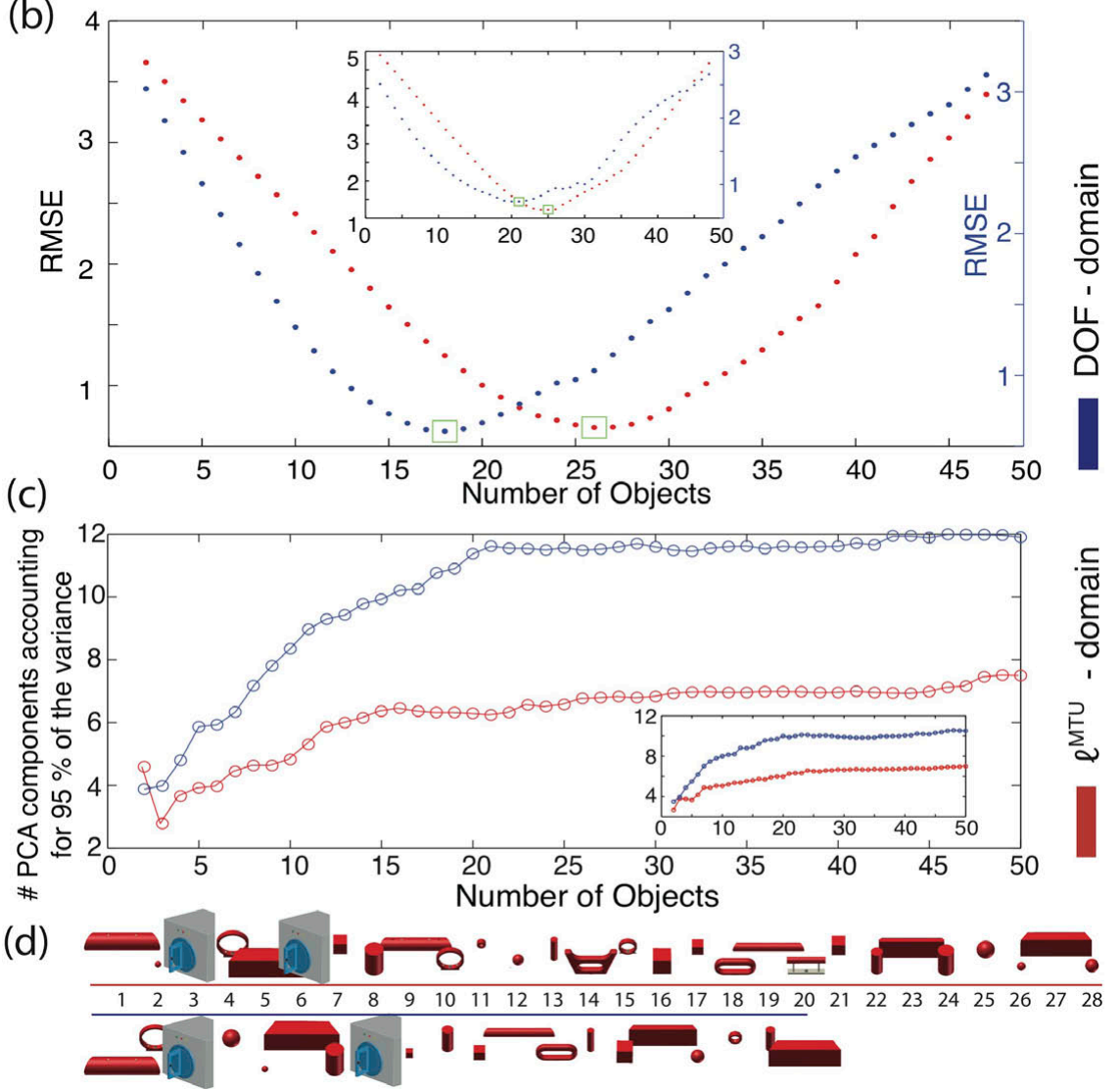

Fig. 6. Object classification accuracy. (a) Classification accuracy of the Bayesian Decoder vs. the number of gradually included objects sorted according to their separability (DOF in blue, $\ell^{M T U}$ in red). Decodings were performed on the complete spaces (solid dots; $27 \mathrm{DOF}$ and $50 \mathrm{l}^{\mathrm{MTU}}$ ) and on the reduced spaces (dashed line; 10 DOF and $\left.10 \mathrm{e}^{\mathrm{MTU}}\right)$. To find the optimal number of objects for the decoder in the complete space, the L-method was applied (Section III-E), which provided two regression lines fitting the data best (solid black and green lines). (b) The edge position (intersection of both regression lines) was systematically varied in order to minimize RMSEs. Object numbers with minimal RMSE are marked with green boxes. (c) The number of principal components required to describe $95 \%$ of data variance were plotted against the number of included objects (DOF-domain in red, $\ell^{M T U}$-domain in blue). (d) For animal $M$, the set of objects that achieved decoding accuracies higher than 95\% (28 in $\ell^{M T U}$ and 20 in DOF space) are illustrated. Their order corresponds to the $x$-axis of a-c. Large panels ac: results for animal $M$; inlays: corresponding results for animal $Z$. 


\section{Discussion}

In this study we presented a musculoskeletal modelling method that could account for the specific upper extremity anatomy in two primates. The animal-specific model was used to investigate grasp actions from a large repertoire of different objects.

This study enabled observing and investigating larger kinematics variability in the primate's arm and hand (Fig. 5) than it was possible in previous studies [5, 12]. Furthermore, it allowed for the first time extracting comprehensive kinematics information in 27 DOFs and 50 MTUs in the arm and hand segments simultaneously $[17,18]$. This resulted in higher classification accuracy than reported in previously published methods that used a nine-DOF, non-anatomical kinematics model in conjunction with electromyography (EMG) signals from 24 muscles [16].

Importantly, results showed that the number of components extracted from MTU kinematics that describe $95 \%$ of the data variability across all grip types was substantially smaller than the number of components extracted from DOFs kinematics for reconstructing the same data variability (Fig. 6(c)). This emphasizes on the non-linear relationship between MTU and DOF kinematics $[18,33]$ and proves its exploration to generate a more compact representation of grasping actions than it was possible with conventional joint kinematics. Further research is needed to investigate whether this may have related neural mechanisms. Supporting results in this direction [5] are providing evidence of direct relationships between single M1-neuron activity and the simultaneous actuation of proximal distal joints in the hand segments, such as wrist pronation/supination and thumb-index aperture. In this context, the relationship between single M1 neuron activity and simultaneous actuation of multiple DOFs might reflect the activation of the underlying muscles spanning proximal and distal joints. This hypothesis is further supported by the fact that M1-neurons have a direct influence on the spinal alpha motor neurons innervating MTUs [5]. The ability of extracting variables reflecting muscle behaviour from reach-to-grasp movements, such as presented here, might help further explain the neural code of grasping.

The compact representation of grasping tasks provided by MTU kinematics also resulted in a decoding method that could account for a larger number of objects than observed using DOF kinematics-based decoders Fig. 6(a). In this, MTU redundancy may play an important role. Multiple MTUs spanning the same DOF may play different roles across grasping tasks. This may contribute to assure good representation of a larger number of objects than it was possible with DOFs only.

Some limitations in our methods should be recognized. The proposed primate-specific model was derived and extended from a human upper extremity model [18] (see Methods section). This model was used as a basis for our study because of the extensive research consistently confirming strong anatomical and morphological similarities in the majority of the upper extremity joints and muscles 
between both species [23, 24, 34]. However, some differences should be acknowledged. While joints move about the same DOFs in both species, their range of motion can differ. To support and stabilize quadrupedal locomotion, the shoulder rotation in NHP is smaller than in humans, whereas the NHP wrist allows for a higher abduction and extension [23]. Differences are also reflected at the muscular level across proximal and distal joints [34]. This results in discrepancies in terms of insertion, origin, and size in muscle groups that are shared across the two species. Furthermore, this results in some muscles not being preserved across the two species [34]. Major differences in proximal muscles include the so called 'climbing muscle' (i.e. the dorsoepitrochlearis muscle), which is not present in humans $[35,36]$. Major differences in distal muscles relate to the macaque extensor digitorum communis group in the hand, which is equivalent to the human's extensor digitorum group. The macaque extensor digitorum communis group differs from the human's in that the four separate tendons are united as far distally as the metacarpal heads, after which they diverge and progress toward all fingers. Overall, morphological differences are related to the habitual quadrupedalism of the macaque, with the constant extension of the wrist and fingers and with larger moment arms in the shoulder muscles [34].

Therefore, the ability of fitting the generic model to each animal specifically played an important role in compensating for the reported morphological discrepancies. Fitting errors were small throughout each performed task, providing evidence that the scaled model not only matched a specific static pose in the monkey, but remained kinematically consistent throughout all performed motor tasks (see Results section; 3.2). This resulted in a scaled model that, despite morphological discrepancies with respect to the primate anatomy, resulted in improved performances in dimensionality reduction and decoding with respect to conventional methods based on DOF kinematics analysis.

Future research will assess whether the availability of a more correct musculoskeletal model may substantially improve dimensionality reduction and decoding results. Future work will also use EMGdriven musculoskeletal modelling methodologies [37, 38] for reconstructing all the transformations from the neural drive onset (i.e. muscle-specific EMG onset) to the generation of functional movement in the upper extremity joints. Combining EMG-driven modelling [37] with in vivo recordings of neuronal activity will enable, for the first time, tracking and monitoring all the major steps taking place along the neuromuscular pathway in primates.

Findings from this study might have important implications in the context of neurorehabilitation technologies. The development of multi-articulated prosthetic hands with fingers and thumb individually actuated by artificial tendons is providing amputees with effective solutions to restore lost grasping capabilities [39, 40]. Our presented methodology could be used to determine the required length changes to be generated in the prosthesis artificial tendons to reconstruct large repertoires of grasping tasks accurately. Importantly, it allows controlling a large number of artificial 
tendons with a low-dimensional controller as suggested by our PCA results (i.e. Results Section 3.4 and Fig. 5). This could bring current PCA-based prosthesis controllers an important step forward [40]. Our proposed methodology could also have implications for the development of advanced neuroprostheses $[8,41]$. In this scenario, recorded neuronal activity might better correlate to the coordinated behaviour of multiple muscles than to the movement of individual DOFs, which might lead to the possibility of controlling tendon-driven prostheses with more intuitive control systems.

\section{Conclusion}

This study showed that a musculoskeletal model of the upper extremity can be scaled to match the specific anatomy and of individual primates. The scaled model provides a non-linear transfer function from the joint domain to the muscle domain, which enables a more compact representation and a higher decoding accuracy of large repertoires of grasping actions, than it was possible using traditional joint kinematics recording. This has direct implications for improving the decoding of complex grasping movements and for the development of biologically-inspired control systems for upper limb prostheses. A better understanding of the inherent non-linearity between the skeletal and muscular systems is crucial for unravelling the nature of the neuromuscular control mechanisms in the primate's arm and hand movement. 


\section{References}

[1] R. D. Martin and A.-E. Martin, Primate origins and evolution: a phylogenetic reconstruction: Princeton University Press Princeton, 1990.

[2] G. Rizzolatti and G. Luppino, "The cortical motor system," Neuron, vol. 31, pp. 889-901, Sep 27 2001.

[3] M. A. Baumann, M. C. Fluet, and H. Scherberger, "Context-specific grasp movement representation in the macaque anterior intraparietal area," The Journal of neuroscience, vol. 29, pp. 6436-48, May 202009.

[4] S. J. Lehmann and $\mathrm{H}$. Scherberger, "Reach and gaze representations in macaque parietal and premotor grasp areas," The Journal of neuroscience, vol. 33, pp. 7038-49, Apr 172013.

[5] C. E. Vargas-Irwin, G. Shakhnarovich, P. Yadollahpour, J. M. Mislow, M. J. Black, and J. P. Donoghue, "Decoding complete reach and grasp actions from local primary motor cortex populations," The Journal of neuroscience, vol. 30, pp. 9659-69, Jul 212010.

[6] S. A. Overduin, A. d'Avella, J. Roh, and E. Bizzi, "Modulation of muscle synergy recruitment in primate grasping," The Journal of neuroscience, vol. 28, pp. 880-92, Jan 232008.

[7] R. N. Lemon, "Descending pathways in motor control," Annual Review of Neuroscience, vol. 31, pp. 195-218, 2008.

[8] J. L. Collinger, B. Wodlinger, J. E. Downey, W. Wang, E. C. Tyler-Kabara, D. J. Weber, et al., "Highperformance neuroprosthetic control by an individual with tetraplegia," Lancet, vol. 381, pp. 55764, Feb 162013.

[9] L. R. Hochberg, D. Bacher, B. Jarosiewicz, N. Y. Masse, J. D. Simeral, J. Vogel, et al., "Reach and grasp by people with tetraplegia using a neurally controlled robotic arm," Nature, vol. 485, pp. 372-5, May 172012.

[10] G. Courtine, M. B. Bunge, J. W. Fawcett, R. G. Grossman, J. H. Kaas, R. Lemon, et al., "Can experiments in nonhuman primates expedite the translation of treatments for spinal cord injury in humans?," Nature Medicine, vol. 13, pp. 561-6, May 2007.

[11] J. Zhuang, W. Truccolo, C. Vargas-Irwin, and J. P. Donoghue, "Decoding 3-D reach and grasp kinematics from high-frequency local field potentials in primate primary motor cortex," IEEE Transactions on Biomedical Engineering, vol. 57, pp. 1774-84, Jul 2010.

[12] V. Aggarwal, M. Mollazadeh, A. G. Davidson, M. H. Schieber, and N. V. Thakor, "State-based decoding of hand and finger kinematics using neuronal ensemble and LFP activity during dexterous reach-to-grasp movements," Journal of neurophysiology, vol. 109, pp. 3067-81, Jun 2013.

[13] K. Ganguly, D. F. Dimitrov, J. D. Wallis, and J. M. Carmena, "Reversible large-scale modification of cortical networks during neuroprosthetic control," Nature neuroscience, vol. 14, pp. 662-7, May 2011.

[14] S. Schaffelhofer and H. Scherberger, "A new method of accurate hand- and arm-tracking for small primates," Journal of neural engineering, vol. 9, p. 026025, Mar 152012.

[15] S. H. Scott, "Apparatus for measuring and perturbing shoulder and elbow joint positions and torques during reaching," Journal of Neuroscience Methods, vol. 89, pp. 119-27, Jul 151999.

[16] S. A. Overduin, F. Zaheer, E. Bizzi, and A. d'Avella, "An instrumented glove for small primates," Journal of Neuroscience Methods, vol. 187, pp. 100-4, Mar 152010.

[17] S. S. Chan and D. W. Moran, "Computational model of a primate arm: from hand position to joint angles, joint torques and muscle forces," Journal of neural engineering, vol. 3, pp. 327-37, Dec 2006.

[18] K. R. Holzbaur, W. M. Murray, and S. L. Delp, "A model of the upper extremity for simulating musculoskeletal surgery and analyzing neuromuscular control," Annals of biomedical engineering, vol. 33, pp. 829-40, Jun 2005.

[19] R. V. Gonzalez, T. S. Buchanan, and S. L. Delp, "How muscle architecture and moment arms affect wrist flexion-extension moments," Journal of biomechanics, vol. 30, pp. 705-712, 1997.

[20] K. M. Steele, M. C. Tresch, and E. J. Perreault, "The number and choice of muscles impact the results of muscle synergy analyses," Frontiers in computational neuroscience, vol. 7, p. 105, 2013. 
[21] N. Ogihara, H. Makishima, S. Aoi, Y. Sugimoto, K. Tsuchiya, and M. Nakatsukasa, "Development of an anatomically based whole-body musculoskeletal model of the Japanese macaque (Macaca fuscata)," American journal of physical anthropology, vol. 139, pp. 323-38, Jul 2009.

[22] A. C. Schouten, E. de Vlugt, F. C. van der Helm, and G. G. Brouwn, "Optimal posture control of a musculo-skeletal arm model," Biological cybernetics, vol. 84, pp. 143-52, Feb 2001.

[23] M. I. Christel and A. Billard, "Comparison between macaques' and humans' kinematics of prehension: the role of morphological differences and control mechanisms," Behavioural brain research, vol. 131, pp. 169-184, 2002.

[24] E. J. Cheng and S. H. Scott, "Morphometry of Macaca mulatta forelimb. I. Shoulder and elbow muscles and segment inertial parameters," Journal of morphology, vol. 245, pp. 206-24, Sep 2000.

[25] S. L. Delp, F. C. Anderson, A. S. Arnold, P. Loan, A. Habib, C. T. John, et al., "OpenSim: open-source software to create and analyze dynamic simulations of movement," IEEE transactions on biomedical engineering, vol. 54, pp. 1940-50, Nov 2007.

[26] National Research Council, Guidelines for the care and use of mammals in neuroscience and behavioral research. Washington, D.C.: National Academies Press, 2003.

[27] H. Scherberger and S. Schaffelhofer, "Modelling of hand and arm position and orientation," U.S. Patent 20130158946 A1, 2013.

[28] C. Pesyna, K. Pundi, and M. Flanders, "Coordination of hand shape," The Journal of neuroscience, vol. 31, pp. 3757-65, Mar 92011.

[29] J. C. Dunn, "A fuzzy relative of the ISODATA process and its use in detecting compact wellseparated clusters," 1973.

[30] H. Scherberger, M. R. Jarvis, and R. A. Andersen, "Cortical local field potential encodes movement intentions in the posterior parietal cortex," Neuron, vol. 46, pp. 347-54, Apr 212005.

[31] M. Controzzi, C. Cipriani, B. Jehenne, M. Donati, and M. C. Carrozza, "Bio-inspired mechanical design of a tendon-driven dexterous prosthetic hand," Conference proceedings : Annual International Conference of the IEEE Engineering in Medicine and Biology Society., vol. 2010, pp. 499-502, 2010.

[32] S. Salvador and P. Chan, "Learning states and rules for detecting anomalies in time series," Applied Intelligence, vol. 23, pp. 241-255, 2005.

[33] M. Sartori, M. Reggiani, A. J. van den Bogert, and D. G. Lloyd, "Estimation of musculotendon kinematics in large musculoskeletal models using multidimensional B-splines," Journal of Biomechanics, vol. 45, pp. 595-601, Feb 22012.

[34] R. R. Ackermann, "Comparative Primate Anatomy," Dissection Manual, pp. 1-64, 2003.

[35] A. B. Howell and W. L. Strauss, "The muscular system," in Anatomy of the rhesus monkey (Macaca Mulatta), C. G. Hartman and W. L. Strauss, Eds., ed New York: Hafner, 1961.

[36] P. Haninec, R. Tomas, R. Kaiser, and R. Cihak, "Development and clinical significance of the musculus dorsoepitrochlearis in men," Clinical anatomy, vol. 22, pp. 481-8, May 2009.

[37] M. Sartori, M. Reggiani, D. Farina, and D. G. Lloyd, "EMG-driven forward-dynamic estimation of muscle force and joint moment about multiple degrees of freedom in the human lower extremity," PloS one, vol. 7, p. e52618, 2012.

[38] M. Sartori, L. Gizzi, D. G. Lloyd, and D. Farina, "A musculoskeletal model of human locomotion driven by a low dimensional set of impulsive excitation primitives," Frontiers in computational neuroscience, vol. 7, p. 79, 2013.

[39] C. Cipriani, M. Controzzi, and M. C. Carrozza, "The SmartHand transradial prosthesis," Journal of neuroengineering and rehabilitation, vol. 8, p. 29, 2011.

[40] G. C. Matrone, C. Cipriani, M. C. Carrozza, and G. Magenes, "Real-time myoelectric control of a multi-fingered hand prosthesis using principal components analysis," Journal of neuroengineering and rehabilitation, vol. 9, p. 40, 2012.

[41] M. Velliste, S. Perel, M. C. Spalding, A. S. Whitford, and A. B. Schwartz, "Cortical control of a prosthetic arm for self-feeding," Nature, vol. 453, pp. 1098-101, Jun 192008. 



\subsection{From vision to action: a comparative population study of hand grasping areas AIP, F5, and M1}

Translating visual object information into corresponding hand configurations allows us to interact with and influence our environment. In this chapter, the neuronal mechanisms underlying these processes are explored in macaque hand grasping areas AIP, F5, and M1. Comparing the neuronal activity of these highly interconnected areas allowed us, for the first time, to explore and visualize their underlying visuomotor processes at the neuronal population level. We found a predominant role of AIP in encoding visual features of objects whereas F5 bridged the gap to the area M1 that precisely reflected the multidimensional kinematic space. 


\section{From vision to action: a comparative population study of macaque's hand grasping areas AIP, F5, and M1}

From vision to grasping actions

Stefan Schaffelhofer ${ }^{1}$, Hansjörg Scherberger ${ }^{1,2}$

${ }^{1}$ German Primate Center GmbH, D-37077 Göttingen, Germany

${ }^{2}$ Deparment of Biology, University of Göttingen, D-37077 Göttingen, Germany

Correspondence should be addressed to Stefan Schaffelhofer, Kellnerweg 4, D-37077 Göttingen, Germany. Email: sschaffelhofer@dpz.eu

Acknowledgements: The authors thank M. Sartori for developing the musculoskeletal model in collaboration with our lab, R. Ahlert, N. Nazarenus, and L. Burchardt for assistance in animal training, M. Dörge for technical assistance, L. Schaffelhofer for glove production, B. Lamplmair for providing illustrations, and J. Michaels and M. Hepp-Reymond, for their helpful comments on an earlier version of the manuscript. This work was supported by the BMBF (BCCN II, DPZ 01GQ1005C). 


\section{Abstract}

Hand grasping requires the transformation of visual object information into corresponding hand actions. In the primate brain these processes are linked to area AIP (anterior intraparietal cortex), F5 (ventral premotor cortex) and the hand area of M1 (primary motor cortex). Although these areas demonstrate selective responses when hand movements are planned or executed, it is up to now unclear how visual and motor information is encoded on the neuronal population level. To address this question, we trained two macaques to grasp up to 50 different objects in a delayed reach-tohold task. In this, we measured the kinematics of hand and arm together with spiking activity recorded from up to 300 neurons using microelectrode arrays. The high variation of visual stimuli and motor responses elicited from this task allowed us separating visual attributes of objects from motor features of the hand. Canonical discriminant- and hierarchical cluster analysis demonstrated a dominant visual role of AIP during both planning and execution epochs. The neural population separated the objects primarily on their shape and secondarily on their size. Furthermore, we found indicators for the processing of object affordances that are relevant for grasping. In contrast to AIP, we could identify a distinct motor role in $\mathrm{F} 5$ that primarily encoded the objects in motor terms. However, the highest similarity to the recorded hand kinematics could be observed at the level of M1. We could visualize and prove that the population of M1 is precisely reflecting the multi-joint space of hand and arm. Together our results demonstrate the distinct roles of AIP, F5 and M1 at the population level. These findings are highly relevant for understanding how visuomotor transformations are processed in the brain.

Keywords: grasping, objects, hand, vision, action, hand-tracking, population, macaque. 


\section{Introduction}

Grasping objects of different shapes and sizes in daily life appears trivial. We can distinguish between thousands of objects (Biederman, 1987) and form our hands according to their geometry in order to hold and manipulate them (Napier, 1956, Smeets and Brenner, 1999). Although such operations seem to be effortless, their underlying neuronal mechanisms are highly complex and require extensive computational resources (Felleman and Van Essen, 1991, Fagg and Arbib, 1998). The cortical network needs to translate high-dimensional visual information of an object into multidimensional motor signals that control the complex biomechanics of the hand and arm.

In the primate brain, these processes are linked to a large extent to the anterior intraparietal (AIP), the ventral premotor (F5), and the primary motor cortex (M1) (Castiello, 2005, Brochier and Umilta, 2007, Davare et al., 2011, Nelissen and Vanduffel, 2011). Within this network, AIP provides access to the dorsal visual stream that processes vision for action (Goodale et al., 1994, Culham et al., 2003). Electrophysiological studies support the hypothesis that AIP extracts visual object information. Neurons in AIP were reported to respond to the presentation of graspable objects (Murata et al., 2000) as well as to 3D contours (Theys et al., 2012) and features of shape (Romero et al., 2014). Additionally, AIP bridges the gap to cortical motor areas via dense reciprocal connections to F5 (Luppino et al., 1999, Borra et al., 2008). This particular part of the fronto-parietal network is associated with the translation of object attributes into motor commands (Jeannerod et al., 1995, Rizzolatti and Luppino, 2001). It has been hypothesized that the extracted visual features of AIP activate motor prototypes in F5 that store hand configurations according to an object's geometry (Rizzolatti and Luppino, 2001). In support of the model, deactivation studies of these areas led to severe deficits in pre-shaping the hand while approaching an object, supporting the model (Gallese et al., 1994, Fogassi et al., 2001). Concurrent electrophysiological studies further suggested that F5 neurons encode objects in motor terms (Raos et al., 2006) and store context specific, objectindependent grip types (Fluet et al., 2010). Connections of F5 to the spinal cord as well as to M1 provides further evidence of the primary role of F5 for grasp movement preparation (Dum and Strick, 2005, Borra et al., 2010).

Although previous studies gave substantial insight into the generation of hand movements, they could not provide a clear separation of visual and motor features at the neuronal population level. Such a differentiation is challenging since the shape of the hand is highly linked to the form of the object being grasped and vice versa. So far, neuronal modulations have been presented that were caused by the presentation of a relatively small set of graspable object (Murata et al., 1997, Murata et al., 2000, Raos et al., 2006, Umilta et al., 2007), which could therefore underlie visual as well as motor processes. Differentiating and explaining the multidimensional visual and motor space 
therefore requires the precise control of visual stimuli and motor responses, a high variability of conditions with many different objects, and the measurement of population of neurons.

In this study, we simultaneously recorded large populations of neurons from area AIP, F5 and M1 using microelectrode arrays while the monkeys grasped up to 50 objects. Furthermore, hand and arm kinematics were recorded with an instrumented glove based on electromagnetic sensors (Schaffelhofer and Scherberger, 2012). Investigating the neural state space revealed a dominant role of AIP for the coding of visual features. Its network separated objects based on their shape, whereas size played a clearly subordinated role. F5 could also present visual features of objects when they were presented, but demonstrated a dominant motor role when grasping movements were planned and executed. In contrast, M1 showed hardly any planning activity, but precisely reflected the multijoint space of the grasping hand.

\section{Methods}

\section{Animal Training}

For this study two rhesus monkeys (macaca mulatta; animals: $Z$ and $M$; sex: female, male; body weight: 7.0 and $10.5 \mathrm{~kg}$, respectively) were trained to grasp a wide range of objects while we recorded hand kinematics and neuronal activity simultaneously in AIP, F5, and M1 (see figure 1 a). During the experiments the animals sat upright in an individual adjusted primate chair with their heads rigidly fixed. In addition the monkeys were habituated to wear an instrumented glove (Schaffelhofer and Scherberger, 2012) that was attached to their grasping hand (contralateral to recording hemisphere). For each step of the training, we applied positive reinforcement training that was adapted to the needs and the learning curve of each animal (Laule et al., 2003). Animal care and all experimental procedures were conducted in accordance with German and European laws governing animal care and were in agreement with the guidelines for the care and use of mammals in neuroscience and behavioural research (National Research Council, 2003) .

\section{Experimental Setup.}

For this study we developed an experimental setup that allowed us to present a large number of graspable objects to the monkeys while monitoring their behaviour and hand kinematics. During a single recoding session, the animals grasped and subsequently lifted a total of $42-48$ objects of equal weight that were placed onto 8 disposable PC-controlled turntables (see figure $1 \mathrm{a}-\mathrm{b}$ ). In order to create a high variation of hand configurations and visual stimuli we used objects of different shapes and sizes including rings (outer diameter of $10 \mathrm{~mm}, 20 \mathrm{~mm}, 30 \mathrm{~mm}, 40 \mathrm{~mm}, 50 \mathrm{~mm}, 60 \mathrm{~mm}$ ), cubes 
(length of $15 \mathrm{~mm}, 20 \mathrm{~mm}, 25 \mathrm{~mm}, 30 \mathrm{~mm}, 35 \mathrm{~mm}, 40 \mathrm{~mm}$ ), spheres (diameters of $15 \mathrm{~mm}, 20 \mathrm{~mm}, 25$ $\mathrm{mm}, 30 \mathrm{~mm}, 35 \mathrm{~mm}, 40 \mathrm{~mm}$ ), cylinders (equal length, diameter of $15 \mathrm{~mm}, 20 \mathrm{~mm}, 25 \mathrm{~mm}, 30 \mathrm{~mm}, 35$ $\mathrm{mm}, 40 \mathrm{~mm}$ ), and bars (equal height and width, depth of $15 \mathrm{~mm}, 20 \mathrm{~mm}, 25 \mathrm{~mm}, 30 \mathrm{~mm}, 35 \mathrm{~mm}, 40$ $\mathrm{mm}$ ). Furthermore, a mixed turntable was holding objects of different shapes of average size. Important for this study, another turntable holding abstract forms was intended to differ visually but to require identical hand configurations. Complementary, the monkeys were trained to grasp one object, a handle, either with a precision grip or a power grip. In this way our task was extended by two more conditions that evoked similar visual but different motor responses. The handle as well as the three-dimensional (3D) objects were placed at a distance of $\sim 25 \mathrm{~cm}$ in front o the animals at chest level.

We used several sensor technologies for detecting correctly or incorrectly performed grasping trials. Similar to previous works (Baumann et al., 2009, Fluet et al., 2010), we assembled the handle with a photoelectric barrier and a force sensor to detect enclosure and pulling of the handle during power grips. Two additional sensors mounted into clearly visible recessions at each side of the handle allowed measuring the contact of the monkey's thumb and index finger when performing precision grips. Similarly, grasping and lifting one of the 3D-objects activated a light barrier installed below the turntable. The turntable position was controlled and monitored using a step motor and an additional infrared light barrier. Furthermore, our task required monitoring the monkey's eye position. For this purpose we used an optical eye tracking system (model AA-EL-200; ISCAN Inc.). All behavioural and task relevant parameters such as eye-position, the state of the sensors, and the activation of stimuli were controlled using a custom-written behavioural control software implemented in LabVIEW Realtime (National Instruments).

For monitoring the monkeys' hand and arm kinematics, we used an electro-magnetic tracking system (model Wave; Northern Digital Inc.). Conductive materials close to the systems' field generator can lead to distortions of the electro-magnetic field and to subsequent inaccuracies of the measurement (Raab et al., 1979, Kirsch et al., 2006). For this reason, we built the setup without the use ferromagnetic materials and used plastics, fiberglass or wood instead. Furthermore, we placed the step motor driving the turntable $1 \mathrm{~m}$ away from the field generator and connected it with fan belts to the turntable axis. 
a
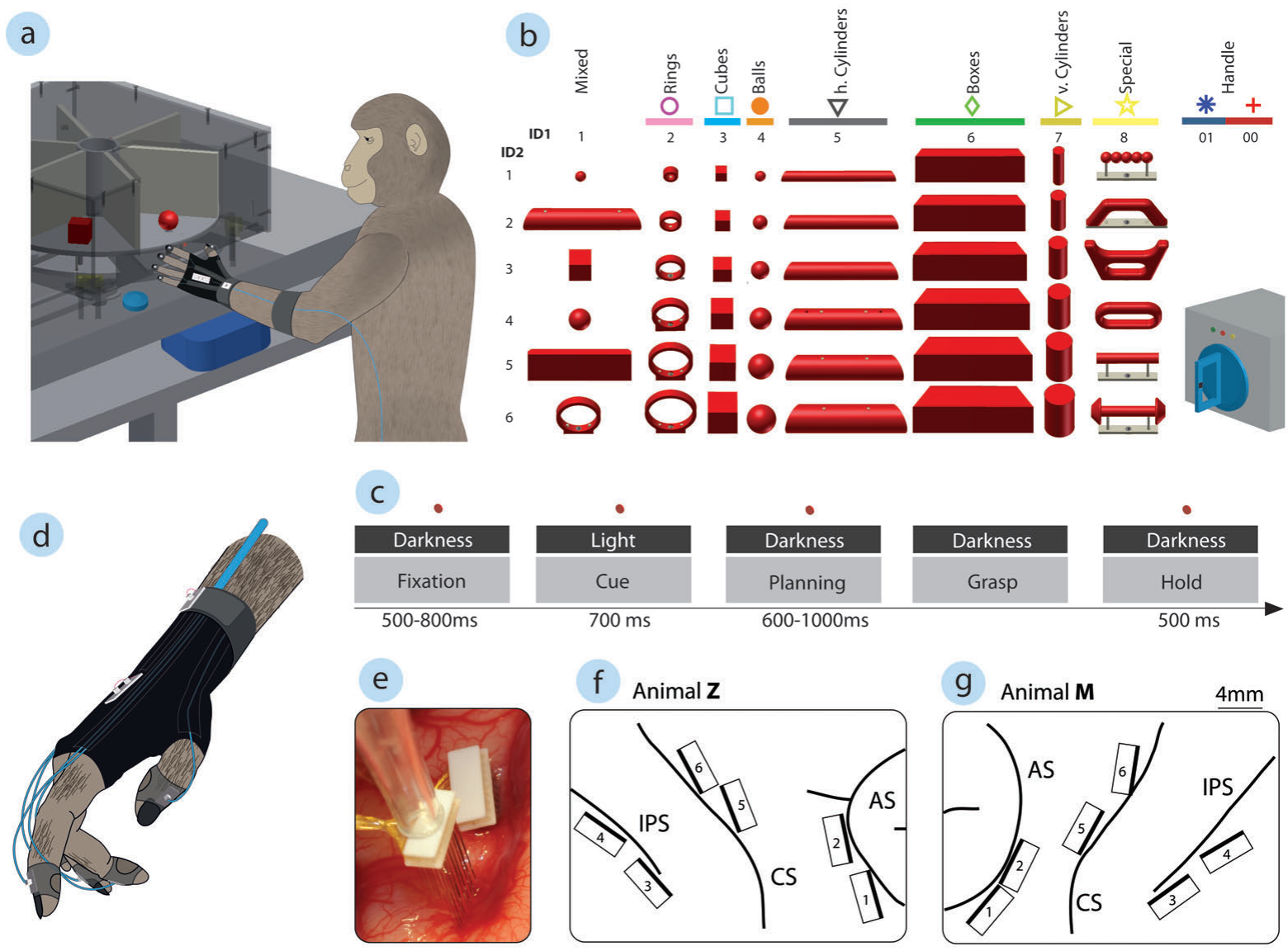

Figure 1. Behavioural design and implantation details. (a) Two macaque monkeys were trained to grasp $3 D$ objects that were presented on a PC-controlled turntable in a pseudorandom order. (b) In total, 48 objects of different shapes and sizes were mounted onto 8 exchangeable turntables and presented to and subsequently grasped by the animal. Additionally, the monkeys were instructed to perform either precision or power grips on a handle. We denoted each of the 50 grasping conditions with a double-digit number (ID1, ID2), a colour code, and a symbol to allow an easy identification of the large number of objects throughout this manuscript. (c) All grasping actions were performed as a delayed reach-to-grasp task consisting of eye-fixation, cue, planning, grasping and hold epochs. (d) An instrumented glove equipped with electro-magnetic sensors allowed monitoring and recording the animals' hand and arm kinematics in 27 DOF. (e-g) Simultaneously, we recorded the activity from large populations of neurons using floating microelectrode arrays implanted into areas AIP, F5, and M1. (e) Each array consisted of 32 individual electrodes having different length to fit the convexity of the sulcus. (f) In animal $M$ and (g) $Z$ two arrays were implanted in each area: toward the lateral end of the intraparietal sulcus (IPS) in AIP, on the bank of the arcuate sulcus (AS) in F5, on the bank of the central sulcus (CS) in the hand area of M1.

\section{Task paradigms}

Monkeys were trained to grasp for a wide range of objects within a delayed reach-to-grasp task (figure $1 \mathrm{c}$ ). While sitting in the dark the animals could initiate (self-paced) a trial by placing their grasping hand (left hand in monkey $\mathrm{Z}$, right hand in monkey $\mathrm{M}$ ) onto a rest sensor that enabled a fixation LED close to the object. Fixating this spot for a variable time (fixation epoch $500-800 \mathrm{~ms}$ ) enabled a spot light that illuminated the graspable object (cue epoch $700 \mathrm{~ms}$ ). After the light was 
turned off the animals had to withhold movement execution (planning epoch 600-1000ms) until the fixation LED blinked for $100 \mathrm{~ms}$. After this, the monkeys released the rest sensor, reached for the object (movement epoch) and held it for half a second (hold epoch). Planning and movement epochs were performed in darkness except for the red LED that the animals had to fixate throughout the task. In trials where the handle was grasped, one of two additional LEDs was presented in the cue epoch indicating the monkeys to perform either precision (yellow led) or power grips (green LED). All correctly executed trials were rewarded with juice and the monkeys could initiate the next trial after a short delay. Error trials were immediately aborted without reward.

\section{Kinematic recordings}

Finger, hand, and arm kinematics from both monkeys were tracked with an instrumented glove for small primates (figure $1 \mathrm{a}, \mathrm{d}$ ) that allowed extracting the position of 18 joints in 27 DOF at a sampling rate of $100 \mathrm{~Hz}$. The method and its underlying computational model were previously described in detail (Schaffelhofer and Scherberger, 2012). In short, seven sensor coils were placed onto the fingernails of digits and thumb, the hand's dorsum and the wrist. The orientation and position provided by each sensor was used in combination with the measured anatomical dimensions to estimate the center of 18 individual joints of the primate upper extremity, including the joints of thumb and digits, the wrist, elbow, and shoulder. We used electro-magnetic sensors since this technology (model WAVE, Northern Digital Inc.) does not depend on line of sight to a camera as required by optical systems (Vargas-Irwin et al., 2010, Aggarwal et al., 2013). This way, we could continuously monitor the monkey' movements, even when their hand was hidden by an object or other obstacles.

As a next step, the recorded joint trajectories were used to drive a 3D-musculoskeletal model (Schaffelhofer et al., 2014) that was scaled to match the specific anatomy of each primate. The model was implemented in OpenSim (Delp et al., 2007) and allowed extracting a total of 27 DOF, including finger flexion/extension, finger adduction/abduction, wrist flexion/extension, wrist adduction/abduction, wrist pronation/supination, elbow flexion, shoulder elevation, shoulder rotation, and shoulder adduction/abduction. The joint angles extracted from the model were resampled at $50 \mathrm{~Hz}$ and lowpass filtered by a Kaiser window finite impulse response filter (passband cutoff: $5-20 \mathrm{~Hz}$ ). All kinematic DOFs were used to describe the hand configurations during the many trials and grip type conditions and to compare them to the neuronal state space. 


\section{Electrophysiological recordings.}

Single and multiunit activity was recorded simultaneously using microelectrode arrays (FMA, Microprobe Inc., Gaithersburg, MD, USA). In each animal we recorded from in total 192 channels of 6 individual arrays implanted into area AIP, F5, and M1 (figure $1 \mathrm{e}-\mathrm{g}$ ). The neural activity was recorded unfiltered at a frequency of $24 \mathrm{kHz}$ and a resolution of 16 bits (model: RZ2 Biosignal Processor; Tucker Davis Technologies, FL, USA) and was synchronously stored to disk together with the behavioural and kinematic data. Raw recordings were then filtered offline (bandpass cutoff: $0.3-7$ $\mathrm{kHz}$ ) before spikes were detected (threshold: $3.5 \mathrm{x} \mathrm{std}$ ) and extracted. Spike sorting was processed in two steps: First, we applied super-paramagnetic clustering (Quiroga et al., 2004) and later revised the results by visual inspection using Offlinesorter (Plexon, TX, USA) to detect and remove neuronal drift and artifacts.

\section{Surgical procedures and imaging}

Prior to surgery, we performed a 3D anatomical MRI scan of the animal's skull and brain to locate anatomical landmarks (Schaffelhofer et al., in press). For this, the animal was sedated (e.g. $10 \mathrm{mg} / \mathrm{kg}$ ketamine and $0.5 \mathrm{mg} / \mathrm{kg}$ xylazine, i.m.), placed in the scanner (GE Signa HD or Siemens TrioTim; 1.5 Tesla) in a prone position, and T1-weighted images were acquired (iso-voxel size: $0.7 \mathrm{~mm}^{3}$ ).

Then in an initial procedure, a head post (titanium cylinder; diameter, $18 \mathrm{~mm}$ ) was implanted ontop of the skull (approx. stereotaxic position: midline, $40 \mathrm{~mm}$ ant., 20deg forward tilted) and secured with bone cement (Refobacin Plus, BioMed, Berlin) and orthopedic bone screws (Synthes, Switzerland). After recovery from this procedure and subsequent training with head fixation, each animal was implanted in a second procedure with six floating microelectrode arrays (FMAs; MicroProbes for Life Science, Gaithersburg, MD, USA): two arrays in each area AIP, F5, and M1 (see figure 1e-g). FMAs consisted of 32 non-moveable monopolar platinum-iridium electrodes (impedance: $300-600 \mathrm{k} \Omega$ at 1 $\mathrm{kHz}$ ) as well as two ground and two reference electrodes (impedance $<10 \mathrm{k} \Omega$ ). Electrode lengths ranged between 1.5 and $7.1 \mathrm{~mm}$ and were configured as in Townsend et al. (2011).

Electrode array locations are depicted in figure $1 \mathrm{f}-\mathrm{g}$. In both animals the lateral array in AIP was located at the end of the intraparietal sulcus at level of PF, whereas the medial array was placed more posteriorly and medially at the level of PFG (Borra et al. 2008). In area F5, the lateral array was positioned approximately in area F5a (Borra et al. 2009/2010), whereas the medal array was located in F5p in animal $Z$ and at the border of F5a and F5p in animal M. Finally, both arrays in M1 were positioned in the hand area of M1 (anterior bank of the central sulcus at the level of the spur of the arcuate sulcus and medial to it) (Rathelot and Strick, 2009). 
All surgical procedures were performed under aseptical conditions and general anesthesia (induction with $10 \mathrm{mg} / \mathrm{kg}$ ketamine, i.m., and $0.05 \mathrm{mg} / \mathrm{kg}$ atropine, s.c., followed by intubation, 1-2\% isofluorane, and analgesia with $0.01 \mathrm{mg} / \mathrm{kg}$ buprenorphene, s.c.). Heart and respiration rate, electrocardiogram, oxygen saturation, and body temperature were monitored continuously. Systemic antibiotics and analgesics were administered for several days after each surgery. To prevent brain swelling while the dura was open, the animal was mildly hyperventilated (end-tidal $\mathrm{CO}_{2}<30$ $\mathrm{mmHg}$ ) and mannitol kept at hand. Animals were allowed to recover for at least 2 weeks before behavioral training or recording experiments recommenced.

\section{Analysis.}

\section{Peristimulus time histograms and Modulation depth plots}

Peristimulus time histograms (PSTH) were created in order to visualize the activity of example neurons across time and conditions. For this, spike rates were smoothed with a Gaussian Kernel ( $\sigma=$ $50 \mathrm{~ms}$ ) over time and then averaged across trials of the same condition (Baumann et al., 2009). To illustrate the response of a specific condition we used the colour code as introduced in figure $1 \mathrm{~b}$. Complementary, we visualized the modulation depth (MD) of example neurons in specific epochs of interest. The MD between two conditions was defined as their difference in average firing rate. This measure was performed between all possible pairs of conditions and expressed as colour-maps. Additionally, we performed multi-comparison tests to test whether the differences in firing rate between conditions were significant (Anova, Tukey-Kramer criterion, $p<0.01$; Matlab functions: anova1, multcompare).

\section{Sliding ANOVA Population Analysis}

To investigate the population activity during the course of the trial, we tested for significant tuning at multiple time points $t$ using a 1-way ANOVA. Similar to the visualization of PSTH plots we smoothed the spike trains of a neuron with a Gaussian kernel $(\sigma=50 \mathrm{~ms})$ centred around $t$ that was shifted in steps of $1 \mathrm{~ms}$ (sliding window ANOVA, $p<0.01$ ). Due to the variable length of the planning epoch, trials were first aligned to the cue-onset and then also aligned to the grasp-onset.

\section{Dimensionality reduction}

Hand and arm kinematics measured with the instrumented glove (J-space) were recorded synchronously with the neuronal population activity as acquired from the implanted FMAs (N-space). In order to compare and visualize the multiplicity of variables we applied dimensionality reduction methods. For this, we used principal component analysis (PCA) to exploit redundancies (correlations) across variables and to express them in a compact, lower-dimensional fashion. The method creates a 
linear transform of the measured parameters and maps them on a new coordinate system, in which the axes are aligned along orthogonal directions of maximum variation (principal components). This approach was demonstrated to be optimal for describing hand shapes (Pesyna et al., 2011) and was used in this study to reduce and visualize the multidimensional J-space. As an input for PCA, we extracted the joint angles across all trials and DOFs from the hold epoch, which demonstrated the most versatile hand configurations under most stable conditions (input unit: joint angles in degree, input dimensions: trials $x$ DOF).

For exploring the $\mathrm{N}$-space (neuronal population space), we applied canonical discriminant analysis (CDA). Similar to PCA, CDA creates a new transform of the original dataset spanned by linear combinations of the original variables. Whereas PCA creates the new coordinate system in a way that maximizes the total variance, CDA transforms the data in order to maximize the separation between groups (here: task conditions). The first axis in the new transform (first canonical variable) therefore reflects the linear combination of original variables that show the most significant F-statistic in a oneway analysis of variance. The second canonical variable has the maximum separation to it and is aligned orthogonally. In the neuronal state space, CDA explores the directions that are most relevant with respect to the conditions of a task. This way, population activity of a brain area can be presented in a compact, low-dimensional fashion. We performed CDA based on the population vector of the mean firing rates of all neurons and all correctly performed trials in a specific task epoch of interest (e.g. grasp epoch) (input unit: firing rate in $\mathrm{Hz}$, input dimensions: trials $\mathrm{x}$ neurons) with respect to baseline activity. In contrast to other dimensionality reduction methods, CDA considers only variances of the signal related to conditional differences. Due to this advantage, noise or condition irrelevant modulations of the signal get suppressed.

\section{Procrustes analysis}

Procrustes analysis (PCRA) can be used to test similarities or dissimilarities between multidimensional datasets of different measures and scales. In the context of this study, PCRA was used to evaluate resemblance between the joint angle J-space (joint angles) and the $\mathrm{N}$-space (firing rates). For this, we reduced both representations to their lowest common dimension (i.e., 27) using the dimensionality reduction methods explained above. This produced datasets of identical number of trials (e.g., 600) and dimensions (e.g., 27). Then PCRA was used to translate and rotate the space of interest (e.g., N-space of M1 and F5) in a way that minimized the sum of squared distances (SSD) to the corresponding points in the reference space (e.g. J-space) (Matlab function: procrustes). The resulting transform was used to visualize similarities or dissimilarities with respect to the reference space (J-space). As a numerical measure of similarity, the sum of squared distances between the new transform and the reference frame was computed and normalized by the sum of squared 
distances between points of the reference space to their dimensional means (Matlab function: procrustes). This similarity measure s, gives a number between 0 and 1 . Values near 1 imply a high similarity between the multidimensional spaces, whereas values near 0 imply dissimilarity.

\section{Hierarchical Cluster Analysis}

To illustrate and compare the many conditions of our task at the neuronal population level, we performed hierarchical cluster analysis. For this, we extracted the average firing rate of all neurons separately for all conditions and all task epochs of interest (cue and hold epochs). Next, we computed the Mahalanobis distances between the population activities of all possible pairs of task conditions (Matlab function: manova1). This resulting distance matrix (e.g. $50 \times 50$ conditions) was used to create an agglomerative hierarchical cluster tree based on the average linkage criterion (Matlab function: manovacluster). The hierarchical cluster solutions are presented as dendrograms to visualize the similarities/dissimilarities between conditions. 


\section{Results}

Visual features of an object and the configuration of the grasping hand are multidimensional and highly linked to each other. Describing and separating both representations at the neuronal level is therefore challenging and requires a multidimensional approach. To address this question, two macaque monkeys were trained to grasp up to 50 objects while we recorded their hand and arm kinematics from a novel instrumented glove as well as their neuronal activity from 6 microelectrode arrays ( $6 \times 32$ channels). The many objects introduced in this task caused a high variability of both visual stimuli and motor responses, which allowed us to separate visual from motor features at the neuronal population level. In this chapter, we will present the distinct roles of AIP, F5, and M1 for the visuomotor processing of hand grasping movements.

\section{General Population responses}

For this study, we analysed data from 20 recording sessions in two monkeys (10 per animal). On average, spiking activity of $202 \pm 7$ and $355 \pm 20$ (mean \pm s.d) single and multiunits were collected across theses sessions in monkey $Z$ and $M$, respectively. From this, $29.2 \%$ and $25.2 \%$ were recorded from AIP, $37.3 \%$ and $32.3 \%$ from $\mathrm{F} 5$, and $33.5 \%$ and $42.5 \%$ from M1 (animal $\mathrm{Z}$ and $\mathrm{M}$, respectively).

Figure 2 shows the averaged population response of all AIP, F5 and M1 neurons during the task and demonstrates consistency across recording sessions and animals. In accordance with previous studies, neurons in AIP and F5 responded to the visual presentation of graspable objects (Murata et al., 1997, Murata et al., 2000). For both animals, the number of tuned cells in this task epoch was significantly higher in $\operatorname{AIP}(35 \%, 21 \%$, anim. $M$, Z resp.) than in F5 $(28 \%, 13 \%)$, whereas they showed comparable activity in the planning epoch (paired t-test, $p<0.05$ ). Interestingly, the population of AIP neurons responded significantly faster to the presentation of the 3D objects in comparison to F5 (49.7 ms and $54.9 \mathrm{~ms}$, animal $\mathrm{M}, \mathrm{Z}$ resp). As expected, in M1 only a minority of neurons became active in these epochs. However, at the end of the planning epoch preparatory activity could be observed in M1. During movement execution (grasp, hold) M1 presented the strongest response followed by F5 and subsequently AIP (t-test, $p<0.01$ ). Both, M1 and F5 showed a stronger averaged response in the motor than in the cue phase, whereas AIP did not show an activity increase during movement execution in comparison to object presentation ( $t$-test, $\mathrm{p}<0.05)$. 
a

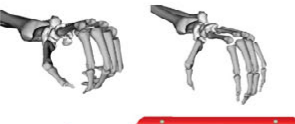

-

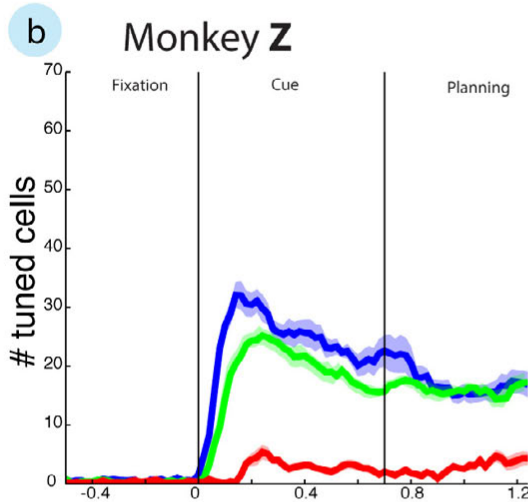

Monkey $\mathbf{M}$

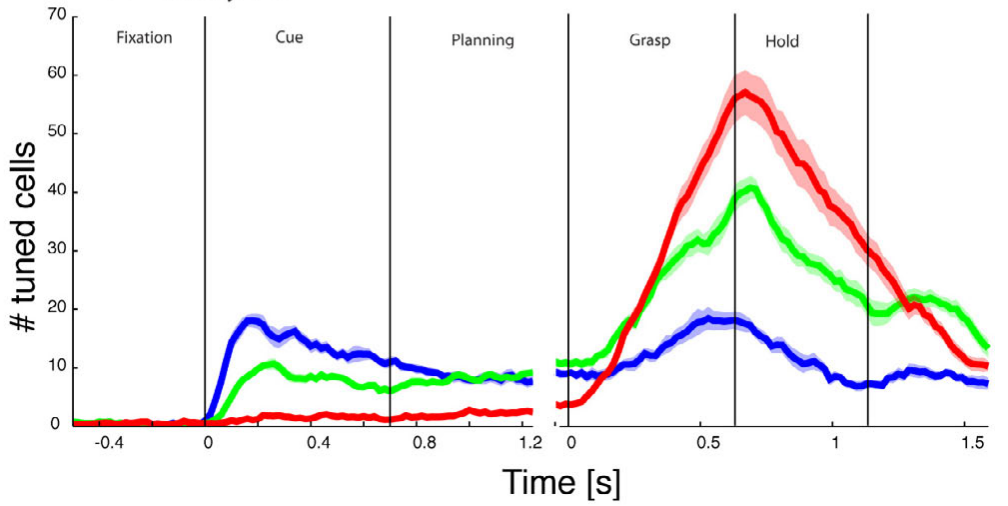

Figure 2. Averaged population activity. (a) Neuronal modulations of area AIP, F5 and M1 were tested on the mixed subset of six objects with a high variability of visual stimuli and motor responses. (b) $A$ one-way analysis of variance was performed on a sliding window along the time-line of trials to observe the percentage of tuned cells. Shades represent the standard error from mean (s.e.m.) across recording sessions. Histograms triggered on the cue and the grasp signal.

Although the results in figure $2 b$ indicate different roles of AIP, F5 and $\mathrm{M} 1$ in visumotor processing, a clear separation of visual and motor features cannot be

addressed from this average analysis: A significant response of a neuron during motor preparation could be related to visual object differences as well as to the subsequent grasping action. Consequently, neuronal responses during the motor epoch could reflect sensory or motor processes.

\section{Vision for Action}

According to the results presented in figure 2, area AIP responded faster and stronger to the presentation of 3D objects than F5 and M1. This fast response was also reflected at the single unit level as demonstrated in the PSTH plots of figure $3 a$ and supplemental figure $1 a$. In accordance with previous studies (Murata et al., 2000), the example neurons showed a vigorous increase in discharge when the graspable objects were presented. Interestingly, the unit displayed in figure 3a was capable of precisely differentiating the individual shapes of the presented objects whereas their size played a subordinated role. To further visualize this attribute, we computed a modulation depth analysis that determined the relative difference in firing rate between all pairs of conditions (see Materials and Methods section). The resulting figure $3 \mathrm{~b}$ revealed a checkered structure caused by the shape-wise order of object conditions 00-76. The maximum modulation depth between all possible pairs of conditions was $62 \mathrm{~Hz}$. To measure the significance of this activity differences, we performed a 
multicomparison test between all conditions (ANOVA and post-hoc Tukey-Kramer criterion, $p<0.01$; Matlab functions: anova1, multcompare). The analysis revealed a high encoding capacity of the example neuron that could significantly separate $71 \%$ of the 946 condition pairs (44 conditions). Interestingly, the neuron decreased its modulation depth in darkness but kept its encoding of shape (objects of similar shape clustered together as indicated in figure 3a).

To analyse the neuronal population, we performed canonical discriminant analysis (CDA see methods section) that allowed reducing the neuronal state space to its most informative dimensions. Figure $3 \mathrm{c}$ and $3 \mathrm{~d}$ illustrate the first three canonical variables during the cue and hold epoch respectively. Each marker represents the neuronal state of an individual trial of the AIP population (see figure $1 \mathrm{~b}$ for symbol and colour code). In the neural state space, objects are separated based on their shapes. Independent of the way the objects were grasped, the neural space accurately separated cylinders, rings, spheres, cubes, and bars. Importantly, this separation could be observed also during the grasp epoch. To quantify these findings, we computed the mahalanobis distance between all pairs of conditions in the complete neural space of AIP (see Materials and Methods). Hierarchical cluster analysis performed on these distance measures confirmed the finding of the CDA and revealed a clear separation of object shapes in the preparatory and execution epochs in animal $Z$, as demonstrated in figure $3 e$ and $3 f$, respectively. Importantly, these finding were consistent in animal $\mathrm{M}$, as shown in supplemental figure 1.

Although AIP showed a primary role in shape separation, we could also observe a minor role in size coding during both preparatory (e.g. cue) and motor epochs (e.g. grasp). As shown in figure 3e-f, the majority of objects were located closest to an object of similar size in the neural state space. The subordinated representation of size in comparison to shape was surprising, since object size has significant influence on the aperture of the grasping hand (Jakobson and Goodale, 1991). 

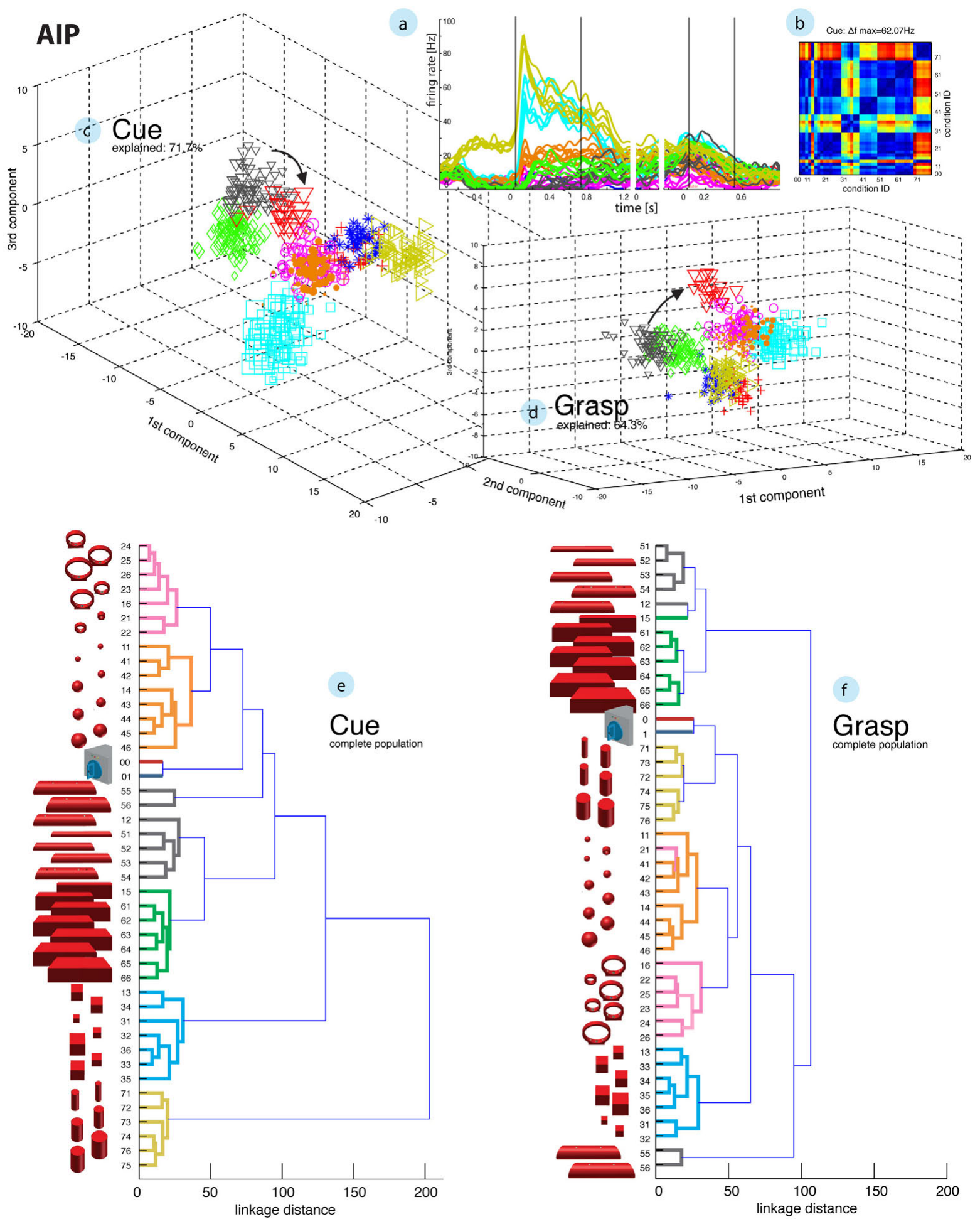

Figure 3. Visual object processing in area AIP. (a) Neurons in AIP responded to the presentation of graspable objects as illustrated by the PSTH-plot of an example neuron. (b) A modulation depth plot highlights the differences in firing rate between conditions in the cue epoch (condition order: 00-01, 11-16, 21-26, 31-36, 41-46, 51-56, 61-66, 71-76). Reddish colours indicate condition pairs of high modulation depth, whereas bluish colours reflect conditions of low modulation depth. (c-d) The shape-wise clustering of objects could be also demonstrated at the population level in the CDA-plots. Arrows indicate a shift in the neuronal space when the big horizontal cylinders (red triangles) were grasped from below. See text. (e-f) Dendrograms derived from hierarchical cluster analysis confirmed the finding on the complete population of AIP-neurons. Symbols and colour code in a, c-f according to figure $1 b$. 
To further test the coding of object shape vs. grip type, we designed six different objects of various shapes (abstract objects) that required equal hand configurations for grasping (see figure 4a). As intended, the equal motor actions caused the population response in M1 to be highly uniform. The number of tuned cells dropped from $68 \%$ to $9 \%$ and from $61 \%$ to $4 \%$ in animal $Z$ and $M$, respectively, when the animals were grasping the abstract (same grip, figure 4) instead of the mixed object set (different grips, figure 2). Strikingly, the response of the AIP population (cue epoch) was not affected by the consistent grip types and was able to separate the abstract objects as well as the mixed ones ( $t$-test, $p>0.05$ ) (see figure $2 b$ and $4 c$ ). Therefore, the observed modulations can be attributed to the object shape.
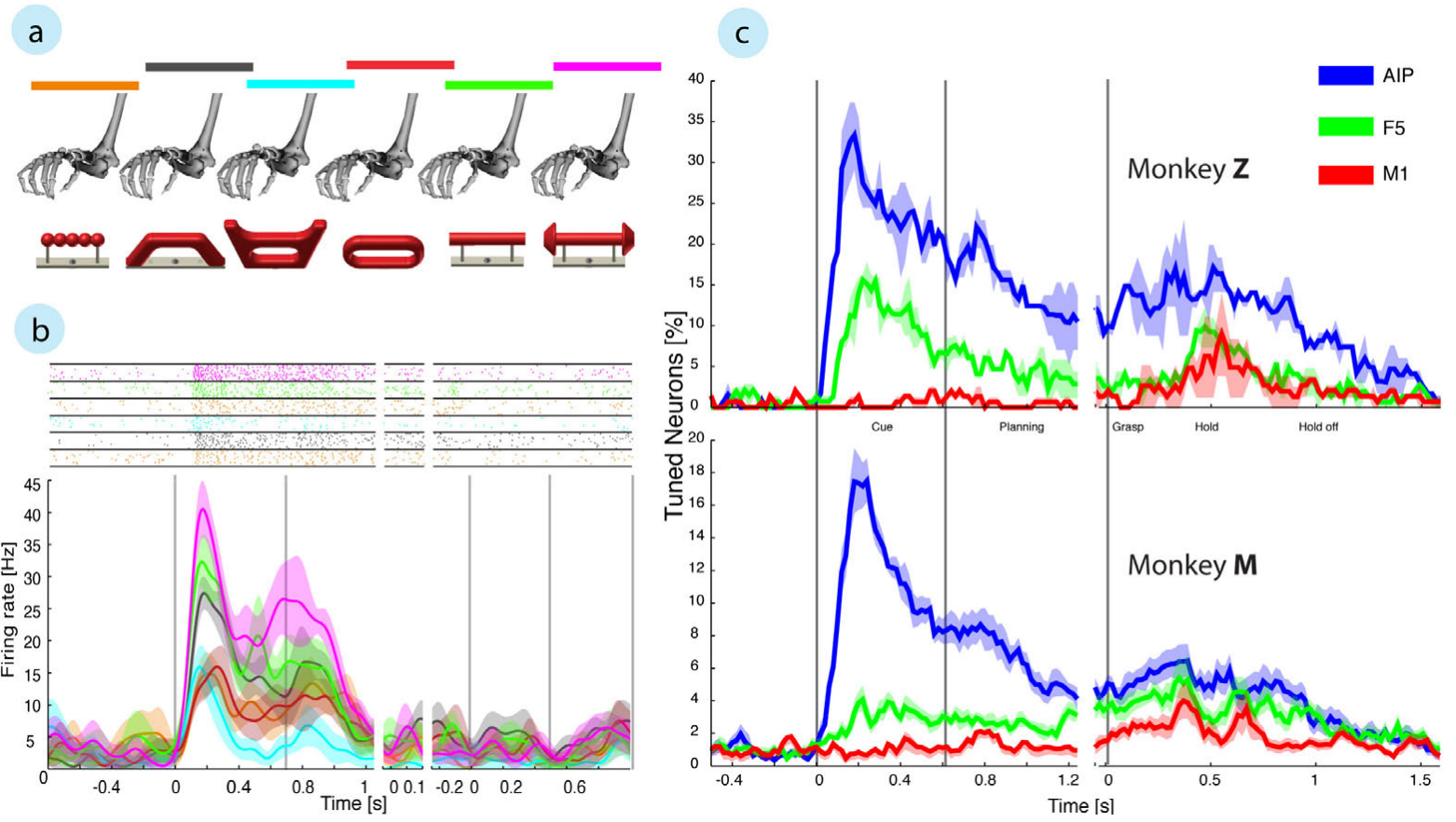

Figure 4. Visual processing of abstract object-shapes. (a) A set of six objects was designed that elicited different visual stimuli but the same motor action. (b) In AIP, single units differentiated these objects as demonstrated by the PSTH of an example neuron. (c) Additionally, a sliding one-way ANOVA was computed to visualize the population response in AIP, F5, and M1.

These results further support the idea that AIP plays a primary role for processing shapes. This hypothesis is also supported by the fact that AIP remained the most tuned area during the planning and movement phases, as shown in figure 4c. However, the number of significantly tuned cells decreased during these epochs in comparison to the responses evoked by the mixed objects (figure $2 b)$. This reduced selectivity could indicate visual transformations that extract object affordances relevant for grasping (Fagg and Arbib, 1998, Rizzolatti and Luppino, 2001). In this theory, AIP could 
visually reduce 3D shapes to its parts relevant for grasping. This would be, in the example of the abstract shapes, the handle that has nearly the same physical dimensions across all six objects.

We found further evidence for this hypothesis when we focused on objects that caused equal visual stimuli but different motor responses. To create such a scenario, the monkeys were trained to perform power or precision grips on the same object, the handle (condition 00 and 01). Although both conditions were located most distant in the kinematic space (see figure $6 \mathrm{c}$ and figure $7 \mathrm{a}$ for detailed information), they were located closest to each other at the population level of AIP in both animals (see figure $3 e-f$, and supplemental figure 1e-f), suggesting a visual representation of the handle. However, statistical analysis on both conditions (00 01) revealed that $21 \%$ and $16 \%$ of AIP neurons in animal $Z$ and $M$ significantly differentiated these conditions (ANOVA tested in grasp epoch, $\mathrm{p}<0.01)$. The clear visual separation of the handle in respect to the other $3 \mathrm{D}$ objects the population level in combination with the subordinated but existing differentiation of power and precision conditions suggests the coding of object affordances rather then the required grip types.

Additional evidence for the processing of object affordances could be observed in monkey $Z$ that grasped the two biggest horizontal cylinders $(55,56)$ in two ways: either from top (pronation) or from below (supination). As demonstrated by the recording shown in figure 3, AIP was capable of differentiating these approaches at the neuronal population level. In more detail, figure 3c-d visualize how these trials (red triangles) separate from the original cluster of cylinders in the neuronal space (arrows indicate the direction of state shift). Two indicators support a visual rather than a motor separation: (1) In the early phase of the cue (0-200 ms after cue onset) all horizontal cylinders were in the same cluster that represented their object shape; (2) after this early visual response, trials started to shift (figure $2 \mathrm{c}$-d), but not into the direction of other objects that required wrist orientation (e.g. vertical cylinders).

Together, we demonstrated strong evidence for a dominant role of AIP for processing object features in visual terms. Distances in the neuronal state space between different objects revealed a primary separation of object shapes and a subordinate role for size coding. Furthermore we found indicators for the coding of object affordances that are highly relevant for the planning and execution of grasping movements.

\section{Motor planning and execution}

To generate grasping movements, visual attributes of objects need to be transformed into adequate motor commands before they get executed (Jeannerod et al., 1995, Rizzolatti and Luppino, 2001); these processes are strongly associated with area F5 and M1, respectively (Murata et al., 1997, Raos et al., 2006, Fluet et al., 2010). For a better understanding of these areas we compared their 
neuronal activity with the 27 DOFs of kinematics data of the macaque' hand that was recorded with an instrumented glove developed for this purpose (Schaffelhofer and Scherberger, 2012, Schaffelhofer et al., 2014).

At the single unit level and in accordance with previous studies, neurons of F5 and M1 where modulated by hand grasping actions as illustrated in figure 5. M1 neurons strongly increased their firing rates during motor execution, but showed minimal modulations during preparatory epochs, such as indicated by the example neuron presented in figure $5 b$ and $d$. This neuron showed a maximum modulation depth of $57 \mathrm{~Hz}$ in the hold epoch (figure 5d, right) and was capable of differentiating $52 \%$ of condition pairs (Anova, Tukey-Kramer criterion, $p<0.01$ ). During the planning epoch, the modulation depth was insufficient to significantly separate any of the conditions. In contrast to this, the planning activity of the F5 example neuron (figure 5a) allowed significantly separating $43 \%$ of condition pairs (figure $5 \mathrm{c}$, left). Furthermore, the neuron's planning activity was highly correlated to its motor execution activity $(r=0.76$, Pearson correlation coefficient between modulation depth maps of planning and grasp epochs).
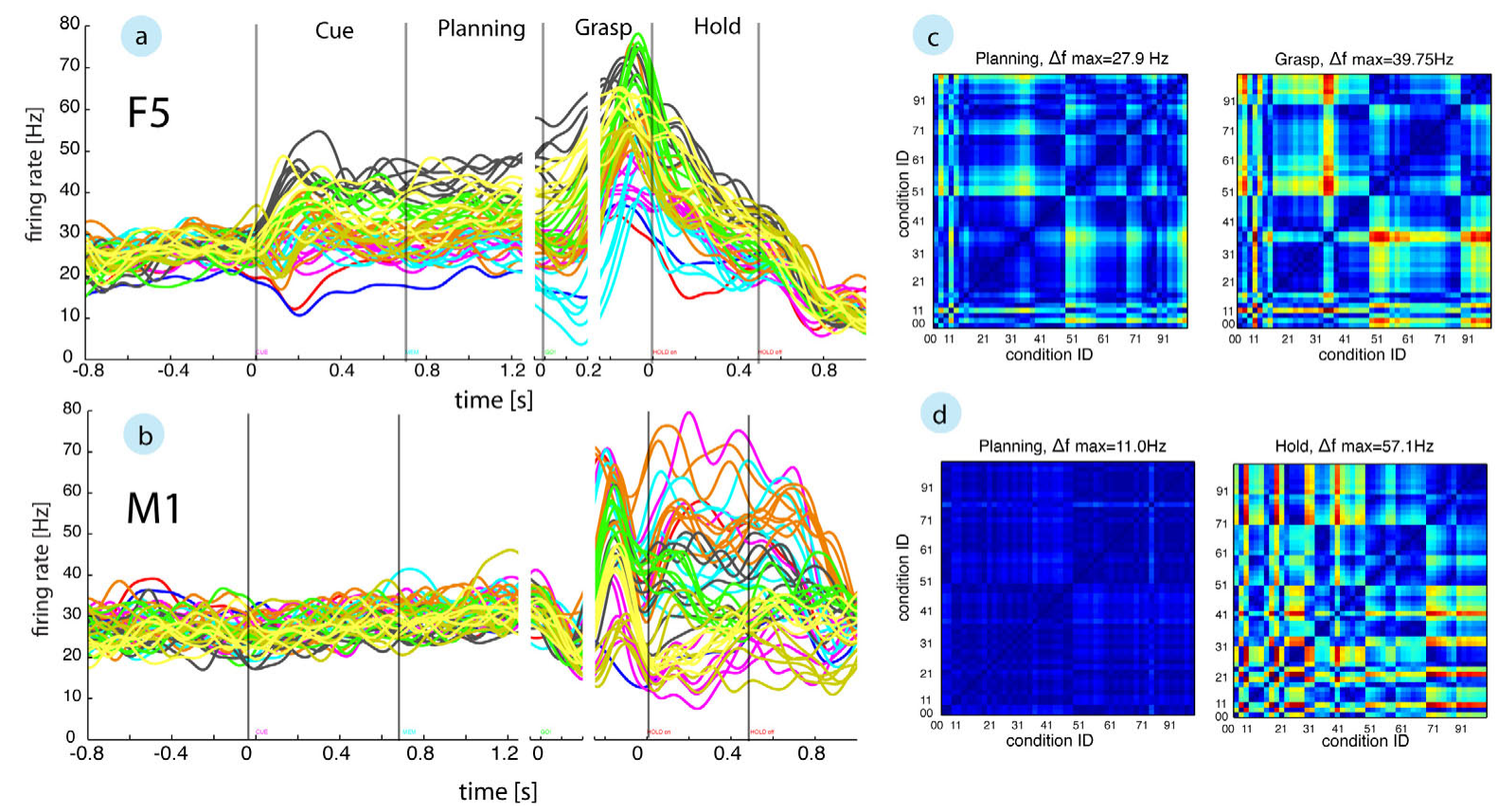

Figure 5. Example units from F5 and M1. (a) and (b) show PSTH responses of two example neurons from F5 and $M 1$, respectively, in monkey M. (c) and (d) show the differences in firing rate between all pairs of conditions as illustrated by modulation depth plots. 
To investigate the relationship between neuronal activity and motor actions, we recorded spiking activity (figure $6 \mathrm{~d}$-e) together with the kinematics of primate hand and arm (figure 6 a-b). For comparing both multidimensional representations, we performed dimensionality reduction methods and procrustes analysis (see Materials and Methods for detailed information).

As a first step in this procedure, we drove a primate specific musculoskeletal model (Schaffelhofer et al., 2014) with the joint trajectories recorded from an instrumented glove developed for this purpose (Schaffelhofer and Scherberger, 2012). As illustrated in figure 6b, we extracted all the DOFs during the hold epoch that provided the highest versatility of hand configurations under the most stable conditions. Performing PCA on this dataset allowed visualizing all correctly performed grips in a low dimensional fashion. Thus, each marker in figure $6 \mathrm{c}$ reflects one individual grip (symbol and colour code according to figure $1 \mathrm{~b}$ ).

In analogy to this analysis, dimensionality reduction was also performed on the neuronal population level. For this we simultaneously recorded the spiking activity from a large population of neurons and extracted the mean firing rates from epochs of interest (e.g. hold epoch) as illustrated in figure 6e. On this dataset we performed CDA and PRCA to reduce and compare the multidimensional N-space (figure 6f) and J-space (figure 6c).

As a first result of dimensionality reduction, the J-space demonstrated a high variability of hand configurations across conditions and closely reflected the hand's wrist orientation ( $1^{\text {st }}$ component) and hand aperture $\left(2^{\text {nd }}\right.$ component). Furthermore the reduced DOF space allowed observing similarities and dissimilarities between objects that were highly relevant for differentiating visual from motor features in the neural space. Here we highlight the most relevant observations:

(1) Objects of small sizes such as the small rings (condition ID 21, 22), spheres $(11,41,42$, ) and cubes $(31,32)$ were grasped similarly using index finger and thumb. Therefore trials of these conditions overlapped or were located close to each other in the reduced- (figure $6 c$, supplemental figure $2 c$ ) and the complete J-space (figure 7a). Please note: symbols of smaller sizes correspond to object of small sizes in figure $6 \mathrm{c}$ and supplemental figure $2 \mathrm{c}$.

(2) Vertical cylinders and big rings $(16,23-26,71-76)$ were grasped in a similar fashion by rotating the wrist and enclosing the corresponding objects with the digits. Therefore, these grips are located close to each other at the population level of the J-space (figure $6 c$, $7 a$, supplemental figure $2 c$ ).

(3) All abstract objects shared a compact cluster and demonstrated their similarity in the J-space (see cluster of yellow star-symbols in figure 6c, and IDs 91-96 in figure 7a). 
(4) Precision and power grips performed on the same handle required highly differentiated hand configurations and were located distant in the J-space (see blue asterix and red cross symbols in figure $6 c$, and IDs 00 and 01 in figure 7a).

(5) The highest separation of hand configuration across objects of similar shape was evoked from the rings that were grasped with precision $(21,22)$ or power grips (23-26). Small rings and big rings are therefore separated in the J-space as illustrated in figure $6 \mathrm{c}$ (see small and big ring symbols in figure $6 c$ and the black cluster highlighted in figure $7 a$.

Importantly, all of these observations could be confirmed in the neural state space of $F 5$ during motor execution epochs (figure $6 \mathrm{f}-\mathrm{g}$ ). In comparison to AIP, conditions of different visual stimuli but equal grips were located close to each other in the neural space (observation 1-3), whereas conditions of similar visual stimuli but different motor responses caused a separation (observation 45). These findings did not only demonstrate a differentiated coding of AIP and F5, but also revealed a primary motor role of F5. These finding were further supported by the high similarity between the J(figure 6c) and $\mathrm{N}$-space (figure 6f) during movement execution.

Furthermore, we used CDA and PRCA in order to visualize F5 planning activity that could be observed at the population and single unit level (see figure $2 b$ and $5 a, c)$. As shown in figure $6 g$ and supplemental figure 2e, F5 neurons demonstrated first expressions of the upcoming motor actions already during the planning epoch. A first manifestation of wrist orientation and grip aperture was reflected by the $1^{\text {st }}$ and $2^{\text {nd }}$ component as indicated in figure $6 \mathrm{f}-\mathrm{g}$. Although the population activity revealed a primary motor coding, individual cells of F5 responded to pure visual features when objects were presented as shown in figure 4c. F5 could therefore be interpreted as an integration area, where visual features are received and translated into motor commands. 


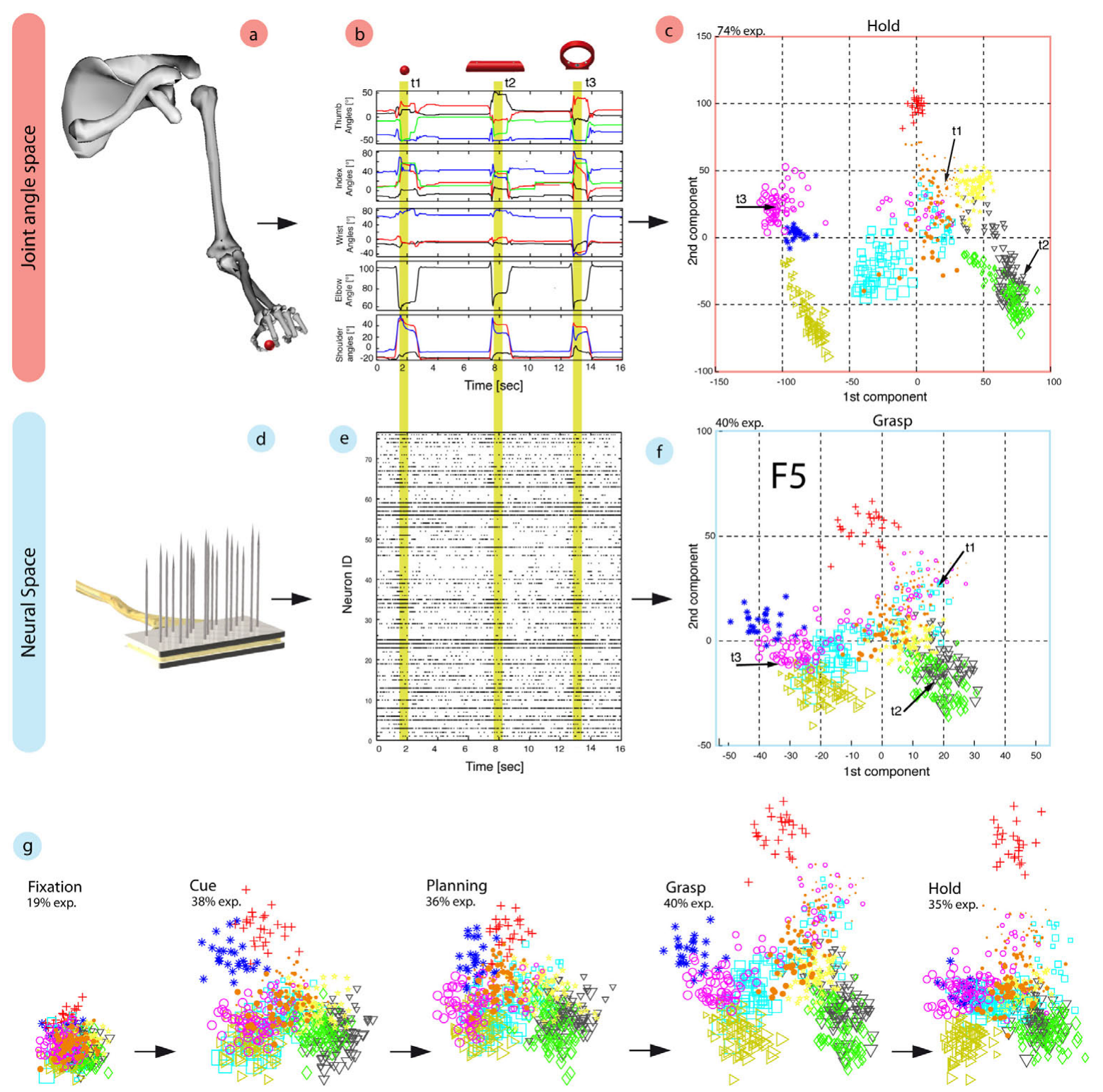

Figure 6. Motor planning and execution in F5. (a) The recorded kinematics were used to drive a monkeyspecific musculoskeletal model that allowed extracting 27 DOF of hand and arm. (b) A selection of DOFs is presented for three sequential grips from thumb and index finger (carpometacarpal adduction/abduction in black, carpometacarpal flexion/extension in red, proximal interphalangeal flexion/extension in blue, distal interphalangeal flexion/extension in green), wrist (abduction/adduction in black, flexion/extension in red, pronation/supination in blue), elbow (flexion in black) and shoulder (adduction/abduction in black, elevation in red, and rotation in blue). (c) PCA performed on the complete DOF-population obtained from the hold epoch allowed visualizing the grip types applied to all objects and trials. (d-e) Complementary, spiking activity from a population of F5 neurons was recorded using microelectrode arrays. The presented raster plots show spiking activity of 76 neurons. (f) The obtained firing rates that represent the neuronal space were transformed with CDA and PCRA to reduce, visualize and compare both multidimensional representations ( $\boldsymbol{c}$ vs. f). The joint angles $\left[{ }^{\circ}\right]$ and firing rates $\left[\mathrm{s}^{-1}\right]$ obtained from example trials are highlighted in yellow $(\boldsymbol{b}, \mathbf{e})$ and marked with arrows in the dimensionality-reduced spaces $(\mathbf{c}, \mathbf{f})$. (g) To visualize the neuronal state space evolution during the course of the task, we performed CDA and PCRA on the spike patterns extracted from fixation, cue, planning, grasp, and hold epochs. For c,f and g: Symbols and colours are used as described in figure $1 \mathrm{~b}$. The size of a symbol corresponds to the actual size of the grasped object. 
To confirm the observations made on the reduced spaces, hierarchical cluster analysis was performed on the complete population of F5 neurons and DOFs. In accordance with the lowdimensional representation, abstract forms, small objects, as well as the big rings and cylinders created individual clusters in the DOF space and were located close to each other. As shown in figure $7 \mathrm{~b}$ and supplemental figure 3 , these motor characteristics were rudimentarily marked in F5 already during motor preparation (see figure $7 \mathrm{~b}$, coloured boxes). These clusters formed during motor planning remained to a large extent during motor execution but increased their relative distance to each other, caused by the higher modulation depth in this epoch (figure 7c).
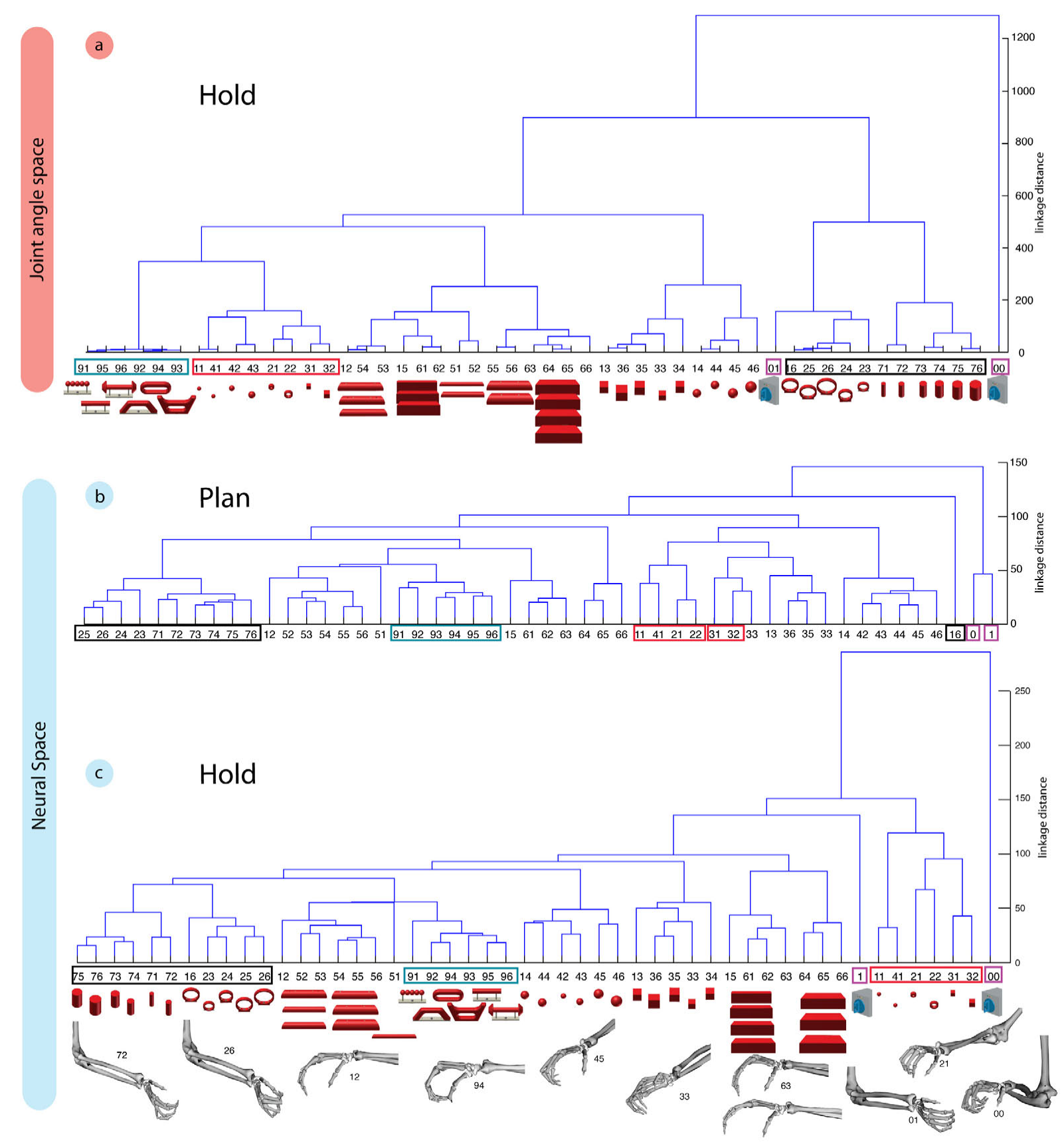

Figure 7. Hierarchical cluster analysis of F5 population. The complete DOF- (a) and F5-populations (b-c) are illustrated as dendrograms for different epochs of the task (plan, hold). A selection of grip types and their corresponding objects are illustrated in (c). Similar motor characteristics are highlighted with coloured boxes. 
Additional indicators for a coding of objects in motor terms was provided by the sliding ANOVA tests presented in figure 2 and 4 . Whereas AIP showed almost constant responses to the presentation of the mixed (different grips) and the abstract set of objects (similar grips), F5 reduced its tuning to the abstract objects significantly from $28 \%$ to $17 \%$ and from $13 \%$ to $4 \%$ for animal $Z$ and $M$ (ttest, $p<0.01)$, respectively. This significant suppression in tuning and the delayed activation of the F5 population in comparison to AIP indicate motor processes that start shortly after an object is presented. However, 17\% and 4\% of all F5 units in monkey $Z$ and $M$ responded visually (cue-epoch) to the presentation of abstract objects, suggesting that F5 contains, at least to a certain extent, cells that temporarily code objects in visual terms.

As expected, M1 neurons showed their strongest modulations when hand movements were executed (PSTH plot in figure 5b; sliding ANOVA in figure 2), but responded equally for the same kind of grip (figure 4c). These findings once again supported the clear and exclusive motor role of M1. However, it has never been demonstrated in which fashion the population of M1 neurons represents the actions of the grasping hand at the population level. The high variability of hand configurations recorded in our task allowed such a description (figure 8, supplemental figure 4). Similar to the analysis performed on the F5 population, PCRA analysis was performed to compare the multidimensional J- and M1-space with each other. Applying these methods revealed strong similarities between the J- (joint angle) Highly important for the understanding of hand movement generation, we found strong similarities between the J- (joint angles) and M1 population (firing rates), as demonstrated in figure $8 \mathrm{a}$ and $8 \mathrm{~b}$, respectively.
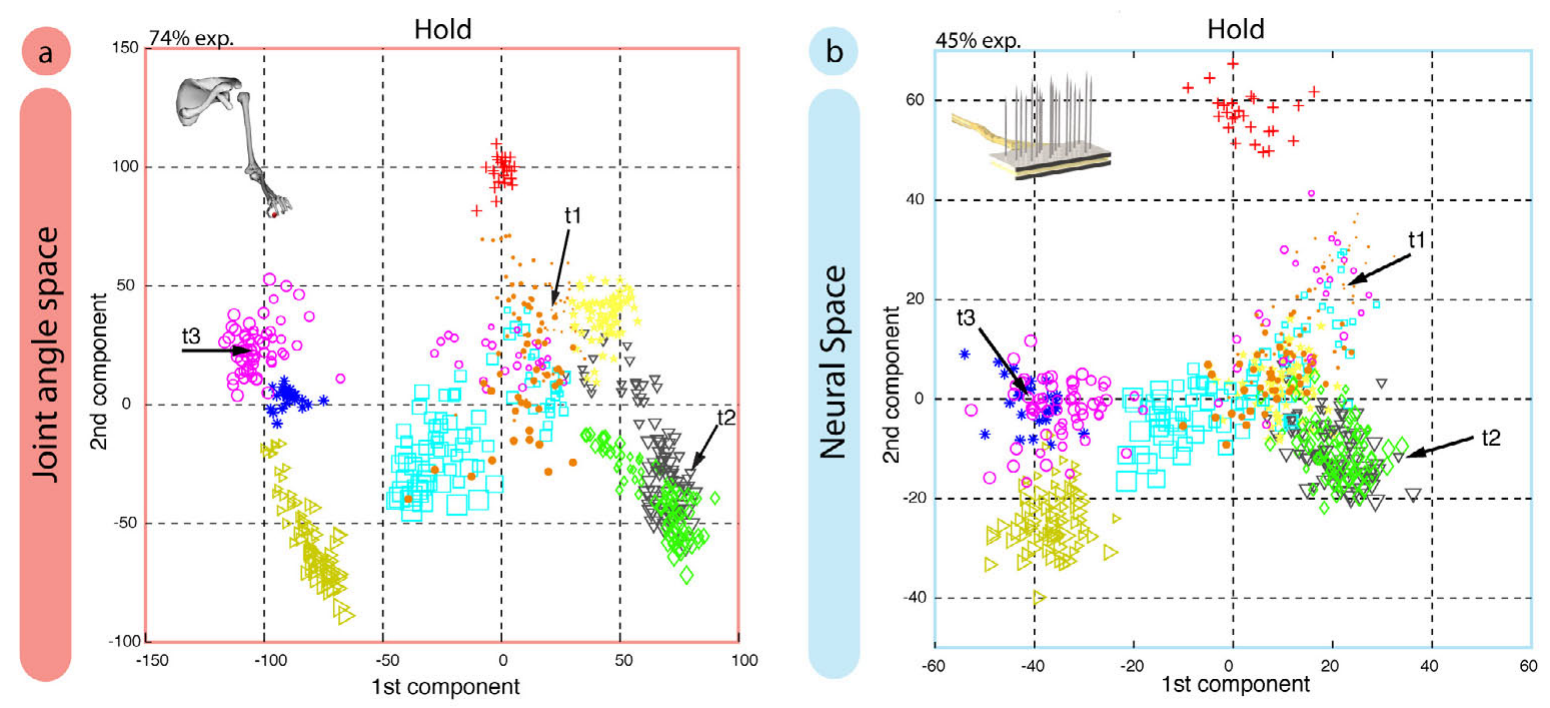

Figure 8. Motor execution in M1. Population activity from (a) the J-space is compared to (b) the N-space of M1 during the hold epoch of the task. Symbols and colours as in figure $1 \mathrm{~b}$. The size of a symbol corresponds to the size of the grasped object. Arrows highlight example trials that correspond to figure 6b,e. 
The conformity of $\mathrm{J}$ and M1 representation was further supported on the complete population as illustrated in the hierarchical cluster trees shown in figure 9 and supplemental figure 3 . The large majority of conditions were assigned to the same clusters in the J- and the M1-space (coloured boxes in figure 9a-b, exceptions: conditions $43,42,1)$. Furthermore, all the 5 motor characteristics defined above could be observed and were even more strongly represented in M1 in comparison to F5: Again, small objects and abstract forms clustered together whereas big and small rings as well as precision and power grips were located distant from each other in the neural space of M1.

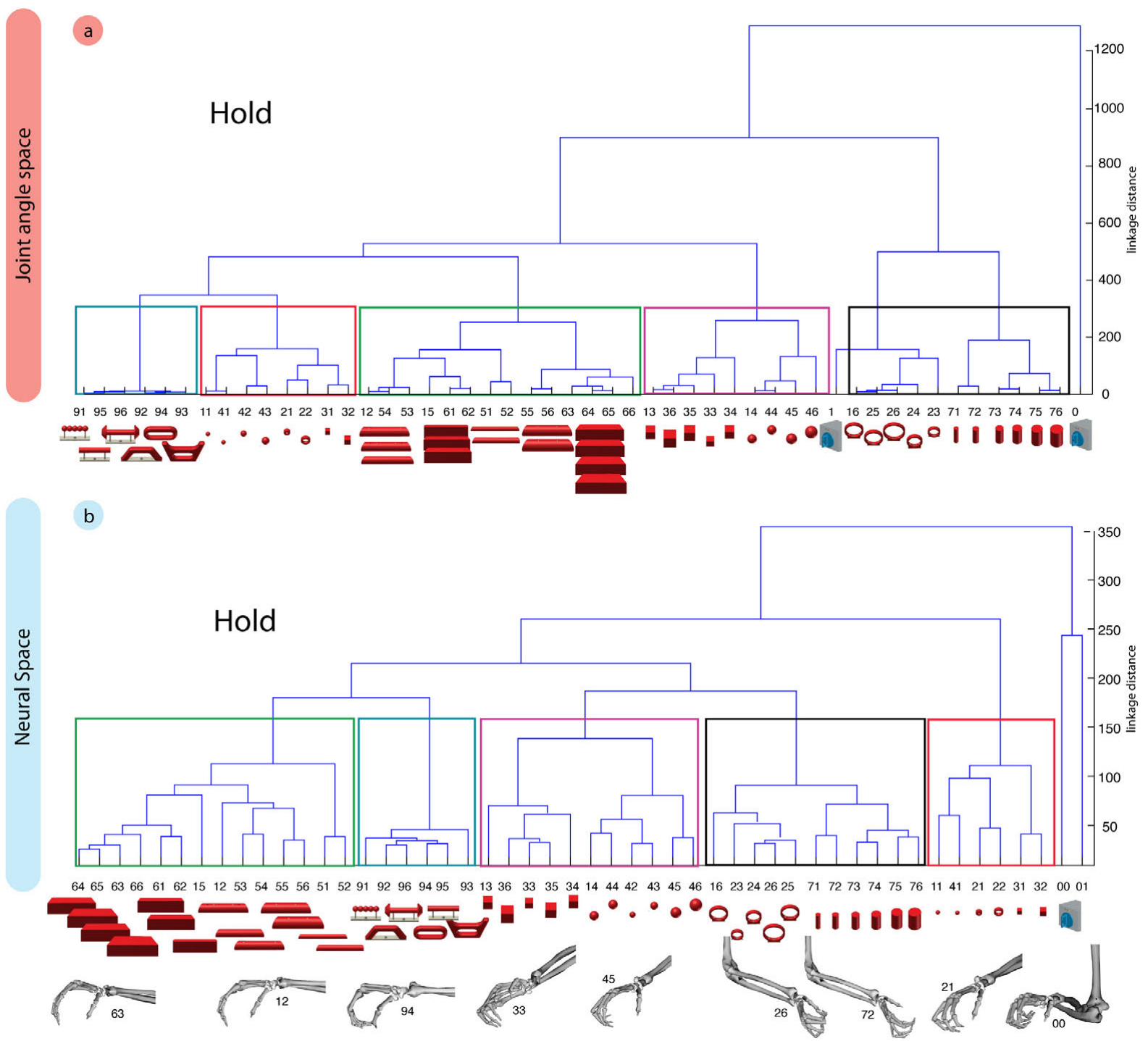

Figure 9. Hierarchical clusters analysis of (a) the J- and the (b) M1-population. Clusters of similar grips are marked with coloured boxes to allow a better comparison of both presentations. 
As a final test, motor conformities, as could be observed from figure 6 and figure 8 , were expressed numerically. For this, similarity measures were performed between the J-space and the $\mathrm{N}$-space of AIP, F5 and M1 using PCRA (see Methods). A similarity of "1" indicates a complete match between two multidimensional spaces over all trials (e.g. $>500$ ) and dimensions (i.e. 27). In contrast, values close to " 0 " would represent high divergences of the two spaces. In accordance with the previous analysis, M1 demonstrated the highest similarity to the J-space $(0.51,0.4$, averaged value over sessions for animal $M$ and $Z$ resp.), followed by F5 $(0.39,0.34$, animal $M$ and $Z$ resp.) and AIP $(0.25$, 0.24 , animal $\mathrm{M}$ and $\mathrm{Z}$ resp.). These findings were consistent over recording sessions and animals as shown in figure 10. Furthermore, differences between areas were significant (ANOVA and post-hoc Tukey-Kramer criterion, $p<0.01)$.

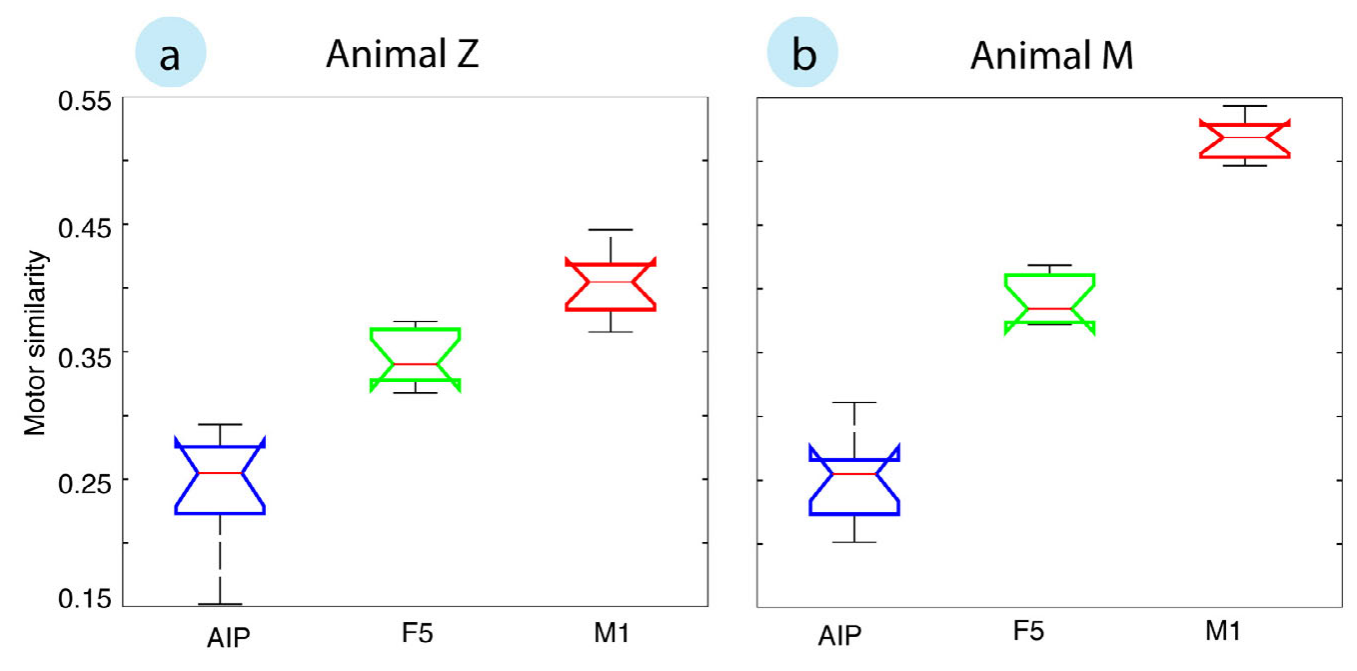

Figure 10. Motor similarity measure. Boxplots illustrate motor similarities in the hold epoch provided by PCRA analysis. Results are shown over all recording sessions for (a) animal Z and (b) animal M in area AIP (blue), F5 (green), and M1 (red). Red lines indicate median value, boxes show lower and upper quartile of data (25\%-75\%), and whiskers indicate maximum values. 


\section{Discussion}

We investigated the neural activity in area AIP, F5, and M1 during a delayed grasping task, in which two macaque monkeys were trained to grasp up to 50 different objects. Using microelectrode arrays in combination with an instrumented glove (Schaffelhofer and Scherberger, 2012, Schaffelhofer et al., 2014) allowed us to simultaneously record the neural activity of many neurons together with the kinematics of hand and arm. The multitude of conditions in this task caused a high variability of visual stimuli and motor responses and allowed separating visual and motor features at the neuronal population level. Area AIP maintained a distinct visual role even during grasp planning and execution epochs (figure 3, figure 4). Its neuronal population primarily separated objects based on their shape while size played a subordinated role. In contrast, the population activity of F5 and M1 reflected the multi-joint space of the hand and arm (figure 6, figure 8). As a consequence, objects in the neuronal space of F5 and M1 were separated based on motor features (figure 7, figure 9).

\section{Recording sites and relation to anatomical connections}

We intended to electrophysiologically investigate the anatomical network for sensorimotor transformation of hand grasping movements. Therefore all microelectrode arrays were implanted under consideration of anatomical studies. AIP receives visual input from parietal visual areas (e.g. LIP, CIP, and V6a) and from the inferior temporal cortex (e.g. TEa, TEm)(Nakamura et al., 2001, Borra et al., 2008). Distinct visual responses of AIP, which were selective for object attributes, were in agreement with these findings (see 3a-b). Furthermore, AIP bridges the gap to the cortical pre-motor areas via reciprocal connections to F5 (Luppino et al., 1999, Borra et al., 2008). Specifically, dense projections have been identified from the lateral convexity of the intraparietal sulcus to the posterior part the inferior arcuate sulcus [see figure 3 Borra et al. (2008)]. In AIP and F5 we found strong visuomotor responses that were modulated while presenting and grasping the 3D objects (see figure 2), which highlighted the importance of these recording sites. Furthermore, F5 has dense connections to the convexity of the hand area of M1 (Dum and Strick, 2005). As demonstrated by Rathelot and Strick (2009), this specific part of M1 consists of direct pathways to alpha-motor neurons in the spinal cord that drive the distal muscles of the upper extremities for hand and finger control. The population responses we recorded from M1 supported these findings: in both monkeys the majority of neurons (see figure 2) was modulated by hand actions, although they required the a similar reaching component. Furthermore, the peak activity of the population of M1 was aligned to the grasp of an object (begin of hold epoch). Together, recordings from the anatomically connected areas AIP, F5 and M1 allowed us to observe and compare their distinct roles at the population level for visuomotor transformation. 


\section{Visual processing for grasping}

In agreement with previous studies (Taira et al., 1990, Murata et al., 2000, Baumann et al., 2009), neurons recorded from AIP were modulated when monkeys observed and grasped 3D objects (figure $2,3)$. Since many of these cells responded selectively to the passive fixation of objects (object type), area AIP has been associated with the coding of objects in visual terms (Fagg and Arbib, 1998, Murata et al., 2000, Rizzolatti et al., 2001). Complementary, cells that were responsive to movements but not to object-fixation (non-object type) were linked to processes that code hand shape or hand orientation (Murata et al., 2000). Since the objects that were used in these studies provided different visual stimuli and motor responses at the same time, object selectivity could reflect visual or motor responses, independent of the task epoch (cue, execution). Furthermore, these studies could not reveal how visual attributes are represented at the population level.

For these reasons we aimed for objects in our task that created similarities and dissimilarities among visual and motor features (see figure 6c, 9a). Investigating these conditions at the neuronal population level of AIP clearly demonstrated a distinct visual separation of objects (figure 3,4) that was not related to the observed motor characteristics as determined by the instrumented glove (figure 6c, 9a). Objects that provided different visual stimuli but the same grips, such as the small objects (conditions $11,41,42,31,32,21,21$ ) or the abstract object set (conditions 91-96) were separated in the neuronal state space of AIP. Furthermore, the well-differentiated precision and power grips performed on the same handle were located close to each other. Importantly, object shape was observed to be the main criteria for object-separation in the neural space, followed by size. This ancillary role in size-coding is remarkable, since this feature is highly relevant for controlling and adapting the aperture of the hand (Jakobson and Goodale, 1991). An explanation for this effect could be that the computational effort (number of neurons) required for processing shape is larger than for size.

To our surprise AIP maintained its visual coding during movement execution in darkness. Similar to the cue epoch, a separation based on shape and size could was observed, although the relative distance between conditions was reduced (figure $3 e, f$ ). We hypothesize, that AIP serves as working memory that stores the visual object information required for motor planning and execution (Rizzolatti and Luppino, 2001). We want to highlight that these findings are related to the population level and do not exclude a motor coding of individual cells of AIP, as suggested in the past (Murata et al., 1997).

Although AIP provided object information throughout the task, we could observe shifts in the neural space when the same objects were grasped differently (see handle and cylinders in figure $3 c, d$ ). Importantly, these modulations were in comparison to F5 and M1 not related to the applied hand 
configurations (see figure 3 and supplemental figure 2, 4 for comparison). These findings suggest that AIP is able to extract visual object information for grasping. This is in agreement with the general understanding of AIP that is associated with the extraction of object affordances (Fagg and Arbib, 1998, Rizzolatti and Luppino, 2001, Baumann et al., 2009, Cisek and Kalaska, 2010).

Finally, the distinct visual role of AIP for the coding of visual object features was in accordance with anatomical studies. Borra et al. (2008) reported dense anatomical connections with the inferotemporal cortex (IT), suggesting that AIP has a unique role in linking the parietofrontal areas responsible for grasping with areas involved in object recognition. In agreement to our finding, neurons of the connected area IT respond selectively to shape and size (Logothetis et al., 1995, Tanaka, 1996).

\section{Motor planning and execution}

For grasping, a visual description of object attributes requires a subsequent transformation into motor commands. F5 is densely connected to AIP (Luppino et al., 1999, Borra et al., 2008) and is associated with these visuomotor processes (Jeannerod et al., 1995, Rizzolatti and Luppino, 2001, Fluet et al., 2010). Similar to AIP, F5-neurons have been reported to respond to the presentation of 3D objects (Murata et al., 1997, Raos et al., 2006). These modulations were first interpreted as object- (Murata et al., 1997) and later as motor representations (Raos et al., 2006). However, corresponding hand kinematics were not measured in these studies.

Here, we could confirm a primary motor role of F5 that reflected well the J-space during motor execution (figure 6, figure 10). Due to the large number of objects tested, we could show for the first time that the population of F5 does not reflect stereotypical grip types (Rizzolatti and Luppino, 2001) but well differentiated configurations of the hand (figure 6). Furthermore, motor characteristics could not only be observed in motor execution epochs, but also during motor preparation (figure 6g, figure $7 \mathrm{~b}$ ). Independent of their shape, objects that required a similar grip (small objects, big rings and vertical cylinders) were located close to each other in the neural space. Importantly and in contrast to AIP, the population of F5 neurons was suppressed already in the cue epoch when abstract object shapes were presented that required the same grip (figure 2 vs. figure 4). This suggests a fast representation of grip features right after object presentation, as proposed by (Raos et al., 2006). However, the population responses in $\mathrm{F} 5$ represented a subgroup of neurons that represented a pure visual coding during the cue epoch (figure 4). We hypothesize that these specific neurons receive the visual input from AIP as required for visuomotor transformation.

In contrast to F5, the hand area of the primary motor cortex showed an exclusive role in motor execution as expected from previous studies (Umilta et al., 2007, Saleh et al., 2010). Furthermore, 
the population of M1 demonstrated strong modulations related to hand and finger control (figure 2, figure 4) in agreement with anatomical, electrophysiological and deactivation studies. (Schieber and Hibbard, 1993, Schieber and Poliakov, 1998, Rathelot and Strick, 2009). Although the bank of the central sulcus has been identified as most relevant for hand movement generation (Rathelot and Strick, 2009), its neuronal population coding for detailed grasping movements has never been described. We demonstrated and visualized, for the first time, that the neuronal space of M1 is precisely reflecting the multi-joint representation of the hand (figure 8 , figure 9 , figure 10). These findings confirm results of decoding studies, which demonstrated significant correlations between M1 neurons (recorded from surface) and kinematic parameters (Vargas-Irwin et al., 2010, Aggarwal et al., 2013).

\section{Visuomotor transformation}

Our findings presented in this study mostly confirmed the visuomotor model proposed by Rizzolatti and Luppino (2001) . We could confirm that AIP is a visual area that processes and stores the attributes of 3D objects. Furthermore, we found indicators for the coding of object affordances in AIP that are highly relevant for grasping. In contrast, F5 coded the objects in motor terms during planning and execution epochs. These findings suggest that visuomotor transformation is achieved by the reciprocal activations of both areas. Finally, strong similarities between F5 and M1 during movement execution suggest a common execution of hand movements that is driven by F5. 


\section{References}

Aggarwal V, Mollazadeh M, Davidson AG, Schieber MH, Thakor NV (2013) State-based decoding of hand and finger kinematics using neuronal ensemble and LFP activity during dexterous reachto-grasp movements. Journal of Neurophysiology 109:3067-3081.

Baumann MA, Fluet MC, Scherberger H (2009) Context-specific grasp movement representation in the macaque anterior intraparietal area. The Journal of Neuroscience 29:6436-6448.

Biederman I (1987) Recognition-by-components: a theory of human image understanding. Psychological review 94:115-147.

Borra E, Belmalih A, Calzavara R, Gerbella M, Murata A, Rozzi S, Luppino G (2008) Cortical connections of the macaque anterior intraparietal (AIP) area. Cerebral cortex 18:1094-1111.

Borra E, Belmalih A, Gerbella M, Rozzi S, Luppino G (2010) Projections of the hand field of the macaque ventral premotor area F5 to the brainstem and spinal cord. Journal of comparative psychology 518:2570-2591.

Brochier T, Umilta MA (2007) Cortical control of grasp in non-human primates. Current opinion in neurobiology 17:637-643.

Castiello U (2005) The neuroscience of grasping. Nature Reviews Neuroscience 6:726-736.

Cisek P, Kalaska JF (2010) Neural mechanisms for interacting with a world full of action choices. Annual review of neuroscience 33:269-298.

National Research Council (2003) Guidelines for the care and use of mammals in neuroscience and behavioral research. Washington, D.C.: National Academies Press.

Culham JC, Danckert SL, DeSouza JF, Gati JS, Menon RS, Goodale MA (2003) Visually guided grasping produces $\mathrm{fMRI}$ activation in dorsal but not ventral stream brain areas. Experimental brain research 153:180-189.

Davare M, Kraskov A, Rothwell JC, Lemon RN (2011) Interactions between areas of the cortical grasping network. Current opinion in neurobiology 21:565-570.

Delp SL, Anderson FC, Arnold AS, Loan P, Habib A, John CT, Guendelman E, Thelen DG (2007) OpenSim: open-source software to create and analyze dynamic simulations of movement. IEEE transactions on biomedical engineering 54:1940-1950.

Dum RP, Strick PL (2005) Frontal lobe inputs to the digit representations of the motor areas on the lateral surface of the hemisphere. The Journal of Neuroscience 25:1375-1386.

Fagg AH, Arbib MA (1998) Modeling parietal-premotor interactions in primate control of grasping. Neural networks 11:1277-1303.

Felleman DJ, Van Essen DC (1991) Distributed Hierarchical Processing in the Primate Cerebral Cortex. Cerebral Cortex 1:1-47.

Fluet MC, Baumann MA, Scherberger H (2010) Context-specific grasp movement representation in macaque ventral premotor cortex. The Journal of Neuroscience 30:15175-15184.

Fogassi L, Gallese V, Buccino G, Craighero L, Fadiga L, Rizzolatti G (2001) Cortical mechanism for the visual guidance of hand grasping movements in the monkey: A reversible inactivation study. Brain 124:571-586.

Gallese V, Murata A, Kaseda M, Niki N, Sakata H (1994) Deficit of hand preshaping after muscimol injection in monkey parietal cortex. Neuroreport 5:1525-1529.

Goodale MA, Meenan JP, Bulthoff HH, Nicolle DA, Murphy KJ, Racicot Cl (1994) Separate neural pathways for the visual analysis of object shape in perception and prehension. Current biology 4:604-610.

Jakobson LS, Goodale MA (1991) Factors affecting higher-order movement planning: a kinematic analysis of human prehension. Experimental brain research 86:199-208.

Jeannerod M, Arbib MA, Rizzolatti G, Sakata H (1995) Grasping objects: the cortical mechanisms of visuomotor transformation. Trends in neurosciences 18:314-320.

Kirsch SR, Schilling C, Brunner G (2006) Assessment of metallic distortions of a electromagnetic tracking system. Medical Imaging 2006: Visualization, Image-Guided Procedures, and Display 6141:J1410-J1410. 
Laule GE, Bloomsmith MA, Schapiro SJ (2003) The use of positive reinforcement training techniques to enhance the care, management, and welfare of primates in the laboratory. Journal of Applied Animal Welfare Science 6:163-173.

Logothetis NK, Pauls J, Poggio T (1995) Shape representation in the inferior temporal cortex of monkeys. Current biology 5:552-563.

Luppino G, Murata A, Govoni P, Matelli M (1999) Largely segregated parietofrontal connections linking rostral intraparietal cortex (areas AIP and VIP) and the ventral premotor cortex (areas F5 and F4). Experimental brain research 128:181-187.

Murata A, Fadiga L, Fogassi L, Gallese V, Raos V, Rizzolatti G (1997) Object representation in the ventral premotor cortex (area F5) of the monkey. Journal of Neurophysiology 78:2226-2230.

Murata A, Gallese V, Luppino G, Kaseda M, Sakata H (2000) Selectivity for the shape, size, and orientation of objects for grasping in neurons of monkey parietal area AIP. Journal of Neurophysiology 83:2580-2601.

Nakamura H, Kuroda T, Wakita M, Kusunoki M, Kato A, Mikami A, Sakata H, Itoh K (2001) From threedimensional space vision to prehensile hand movements: the lateral intraparietal area links the area V3A and the anterior intraparietal area in macaques. The Journal of Neuroscience 21:8174-8187.

Napier JR (1956) The Prehensile Movements of the Human Hand. Journal of Bone and Joint SurgeryBritish Volume 38:902-913.

Nelissen K, Vanduffel W (2011) Grasping-related functional magnetic resonance imaging brain responses in the macaque monkey. The Journal of Neuroscience 31:8220-8229.

Pesyna C, Pundi K, Flanders M (2011) Coordination of hand shape. The Journal of Neuroscience 31:3757-3765.

Quiroga RQ, Nadasdy Z, Ben-Shaul Y (2004) Unsupervised spike detection and sorting with wavelets and superparamagnetic clustering. Neural computation 16:1661-1687.

Raab FH, Blood EB, Steiner TO, Jones HR (1979) Magnetic Position and Orientation Tracking System. IEEE Transactions on Aerospace and Electronic Systems 15:709-718.

Raos V, Umilta MA, Murata A, Fogassi L, Gallese V (2006) Functional properties of grasping-related neurons in the ventral premotor area F5 of the macaque monkey. Journal of Neurophysiology 95:709-729.

Rathelot JA, Strick PL (2009) Subdivisions of primary motor cortex based on cortico-motoneuronal cells. Proceedings of the National Academy of Sciences of the United States of America 106:918-923.

Rizzolatti G, Fogassi L, Gallese V (2001) Neurophysiological mechanisms underlying the understanding and imitation of action. Nature Reviews Neuroscience 2:661-670.

Rizzolatti G, Luppino G (2001) The cortical motor system. Neuron 31:889-901.

Romero MC, Pani P, Janssen P (2014) Coding of shape features in the macaque anterior intraparietal area. The Journal of Neuroscience 34:4006-4021.

Saleh M, Takahashi K, Amit Y, Hatsopoulos NG (2010) Encoding of coordinated grasp trajectories in primary motor cortex. The Journal of Neuroscience 30:17079-17090.

Schaffelhofer S, Agudelo-Toro A, Scherberger $\mathrm{H}$ (in press) Decoding a wide range of hand configurations from macaque Motor, Premotor, and Parietal Cortices. The Journal of Neuroscience.

Schaffelhofer S, Sartori M, Scherberger H, Farina D (2014) Musculoskeletal Representation of a Large Repertoire of Hand Grasping Actions in Primates. IEEE Transactions on Neural Systems and Rehabilitation Engineering Advance online publication Retrieved November 25, 2014 doi:101109/TNSRE20142364776.

Schaffelhofer S, Scherberger H (2012) A new method of accurate hand- and arm-tracking for small primates. Journal of neural engineering 9:026025.

Schieber MH, Hibbard LS (1993) How somatotopic is the motor cortex hand area? Science 261:489492.

Schieber MH, Poliakov AV (1998) Partial inactivation of the primary motor cortex hand area: effects on individuated finger movements. The Journal of Neuroscience 18:9038-9054. 
Smeets JB, Brenner E (1999) A new view on grasping. Motor Control 3:237-271.

Taira M, Mine S, Georgopoulos AP, Murata A, Sakata H (1990) Parietal cortex neurons of the monkey related to the visual guidance of hand movement. Experimental brain research 83:29-36.

Tanaka K (1996) Inferotemporal cortex and object vision. Annual review of neuroscience 19:109-139.

Theys T, Srivastava S, van Loon J, Goffin J, Janssen P (2012) Selectivity for three-dimensional contours and surfaces in the anterior intraparietal area. Journal of Neurophysiology 107:995-1008.

Townsend BR, Subasi E, Scherberger H (2011) Grasp movement decoding from premotor and parietal cortex. The Journal of Neuroscience 31:14386-14398.

Umilta MA, Brochier T, Spinks RL, Lemon RN (2007) Simultaneous recording of macaque premotor and primary motor cortex neuronal populations reveals different functional contributions to visuomotor grasp. Journal of Neurophysiology 98:488-501.

Vargas-Irwin CE, Shakhnarovich G, Yadollahpour P, Mislow JM, Black MJ, Donoghue JP (2010) Decoding complete reach and grasp actions from local primary motor cortex populations. The Journal of Neuroscience 30:9659-9669. 

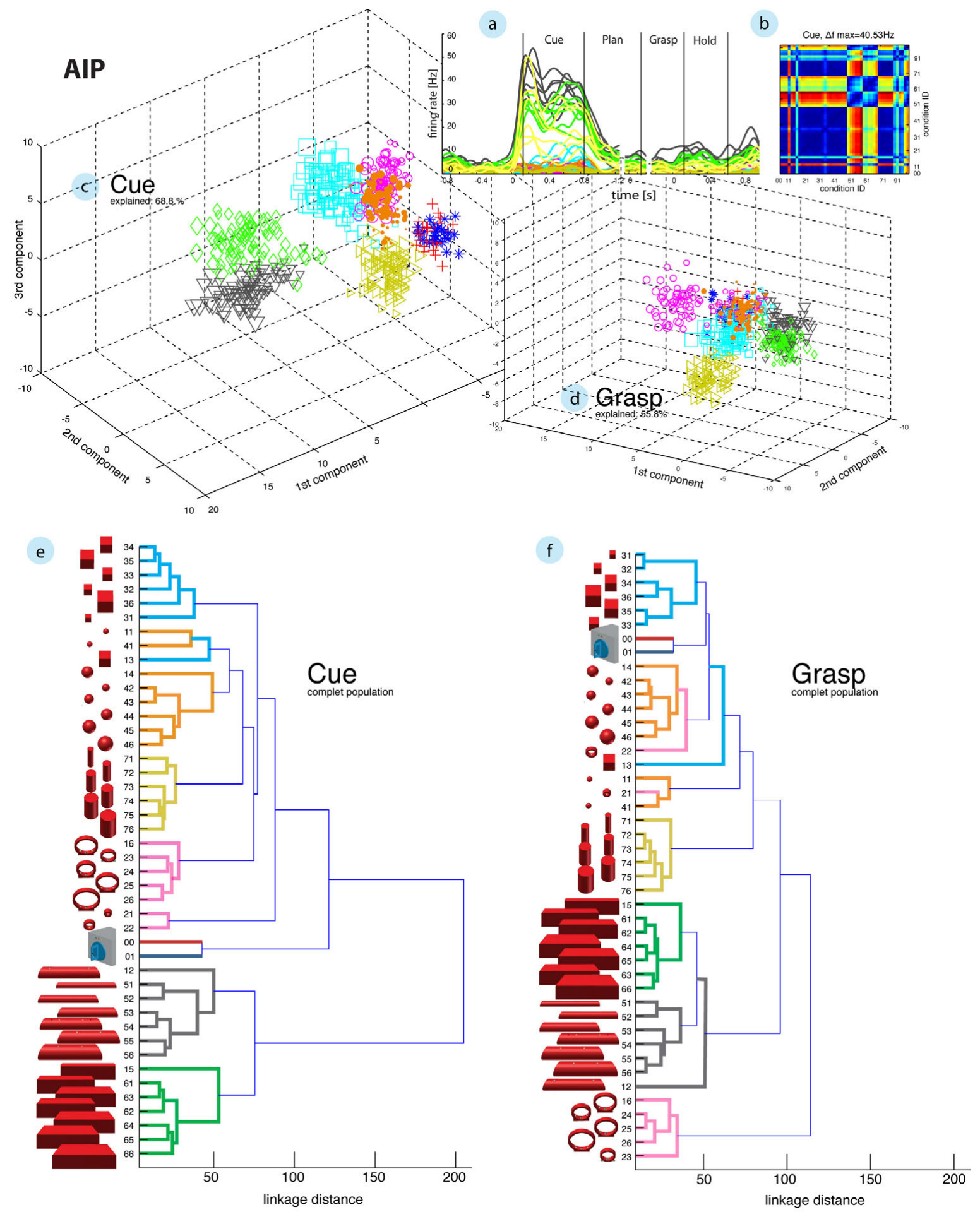

Supplemental figure 1. Visual coding for hand action in AIP in animal M. (a) PSTH plot of AIP example unit. (b) modulation depth plot of example unit during the cue epoch. (c-d) Neural space of AIP during cue and grasp epoch. (e-f) Dendrograms represent complete AIP population activity during the cue and grasp epoch. Symbols and colours as in figure $1 \mathrm{~b}$. 

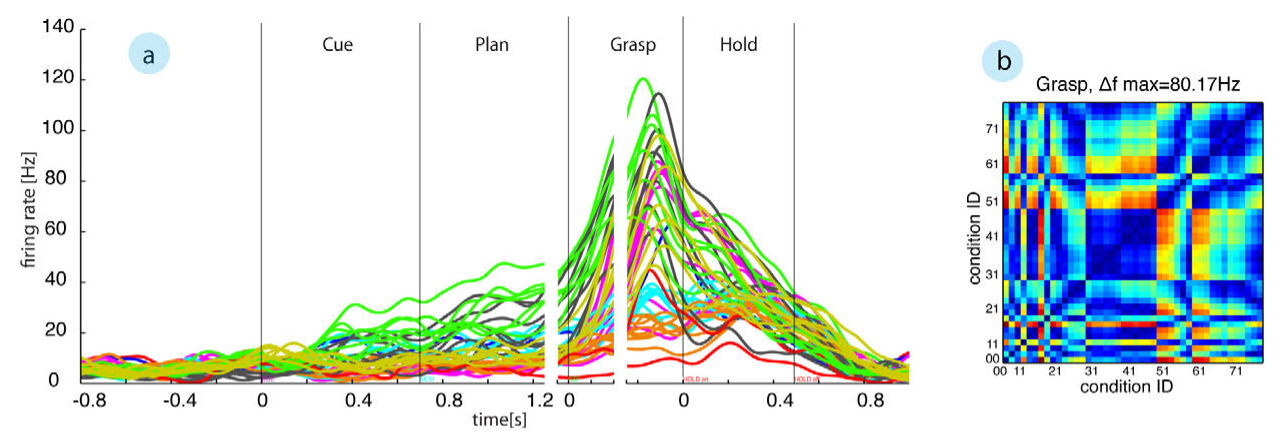

F5
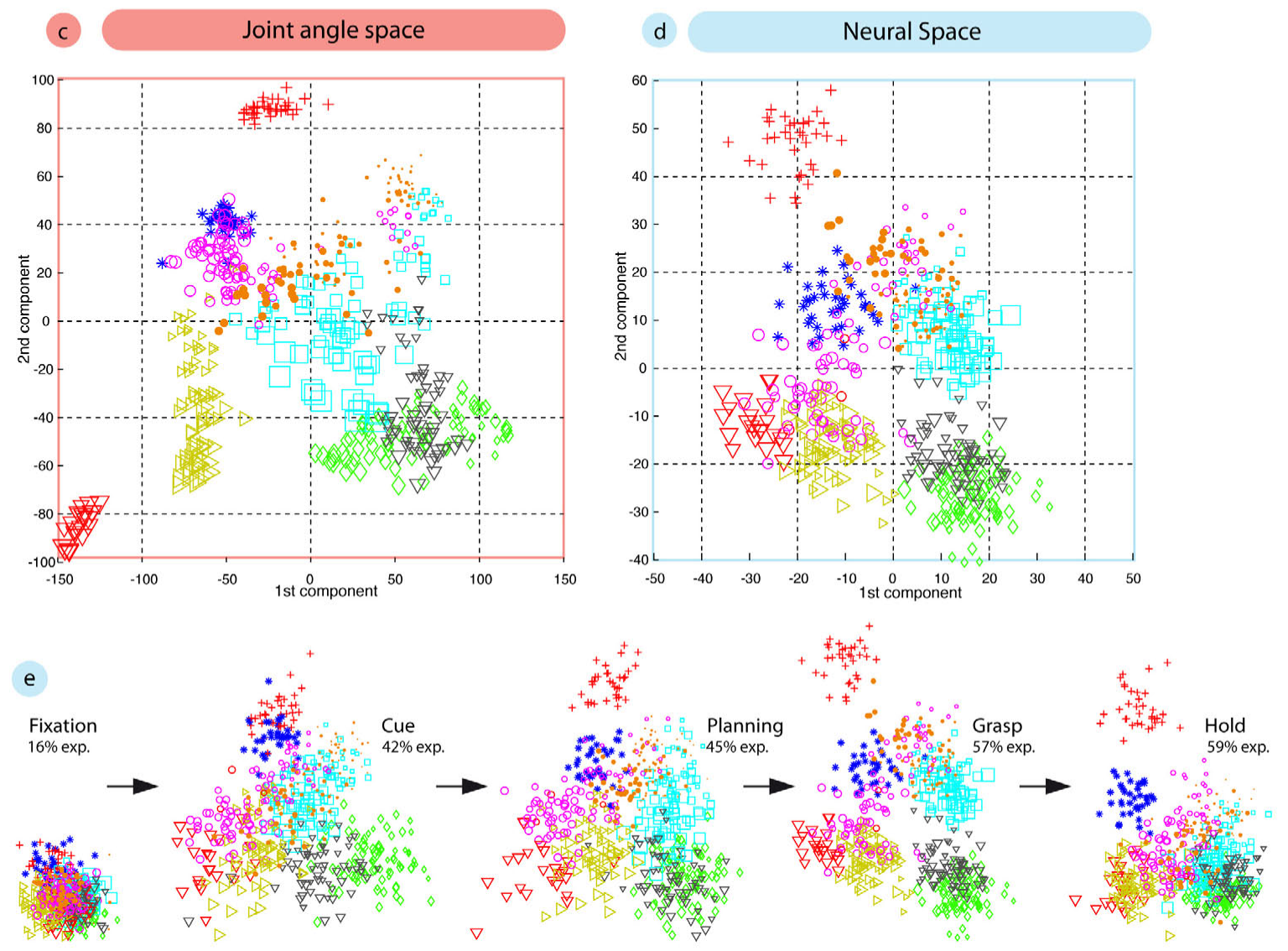

Supplemental figure 2. F5-Motor coding in animal Z. (a) PSTH plot of F5 example unit. (b) modulation depth plot of example unit during grasp epoch. (c) J-space recorded with instrumented glove. (d) Neural space of F5 during grasp epoch. (e) Neural state space evolution during the course of the task. For c-e: Symbols and colours as in figure $1 b$. 


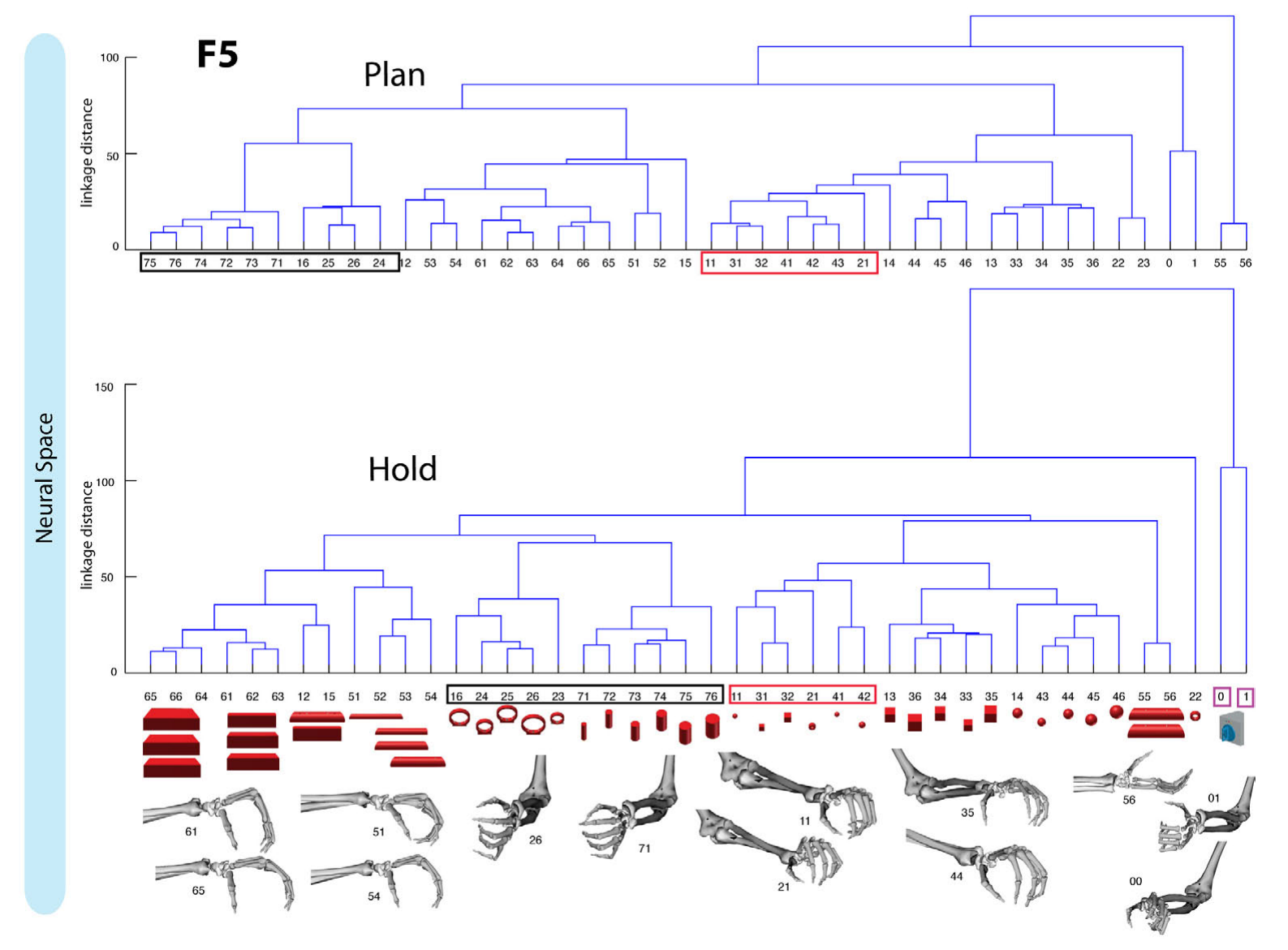

Supplemental figure 3. F5-Hierarchical cluster analysis in animal Z. Dendrograms represents the complete F5 population during plan and the hold epoch. 

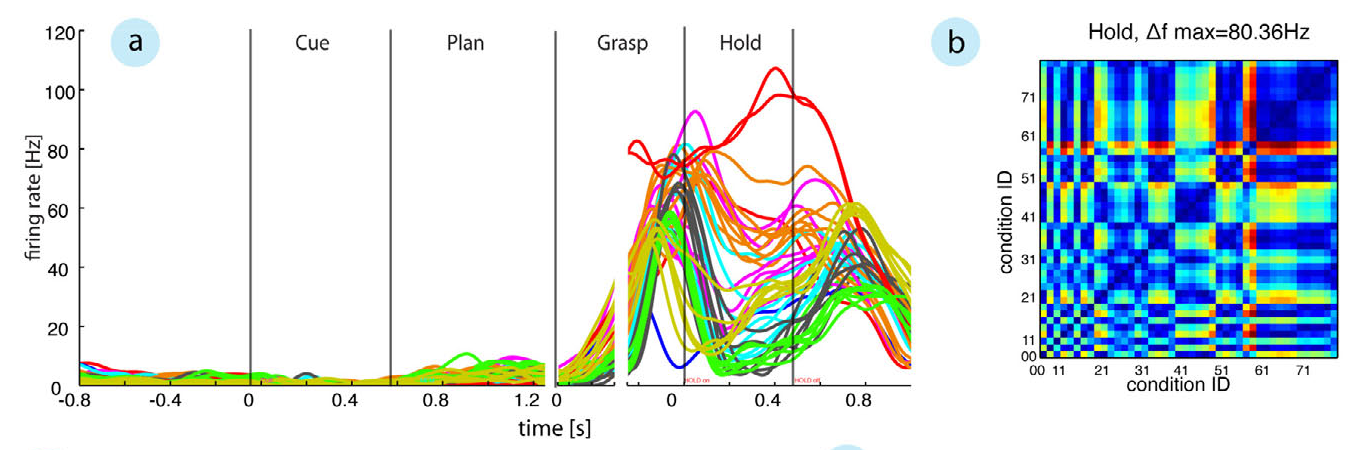

M1
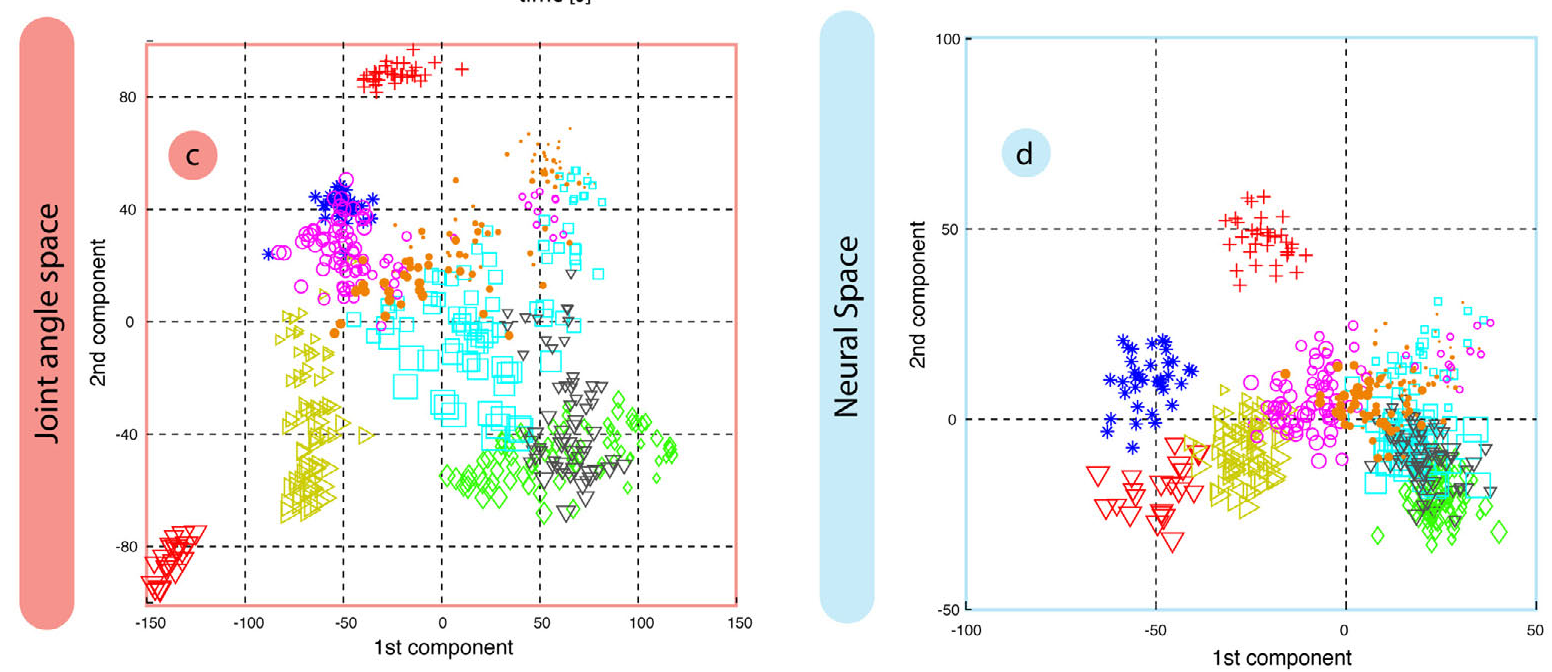

Supplemental figure 4. M1-Motor coding in animal Z. (a) PSTH plot of M1 example unit. (b) modulation depth plot of example unit in grasp epoch. (c) J-space recorded with instrumented glove. (d) N-space of M1 during hold epoch. For c-d: Symbols and colours as in figure 1 b.

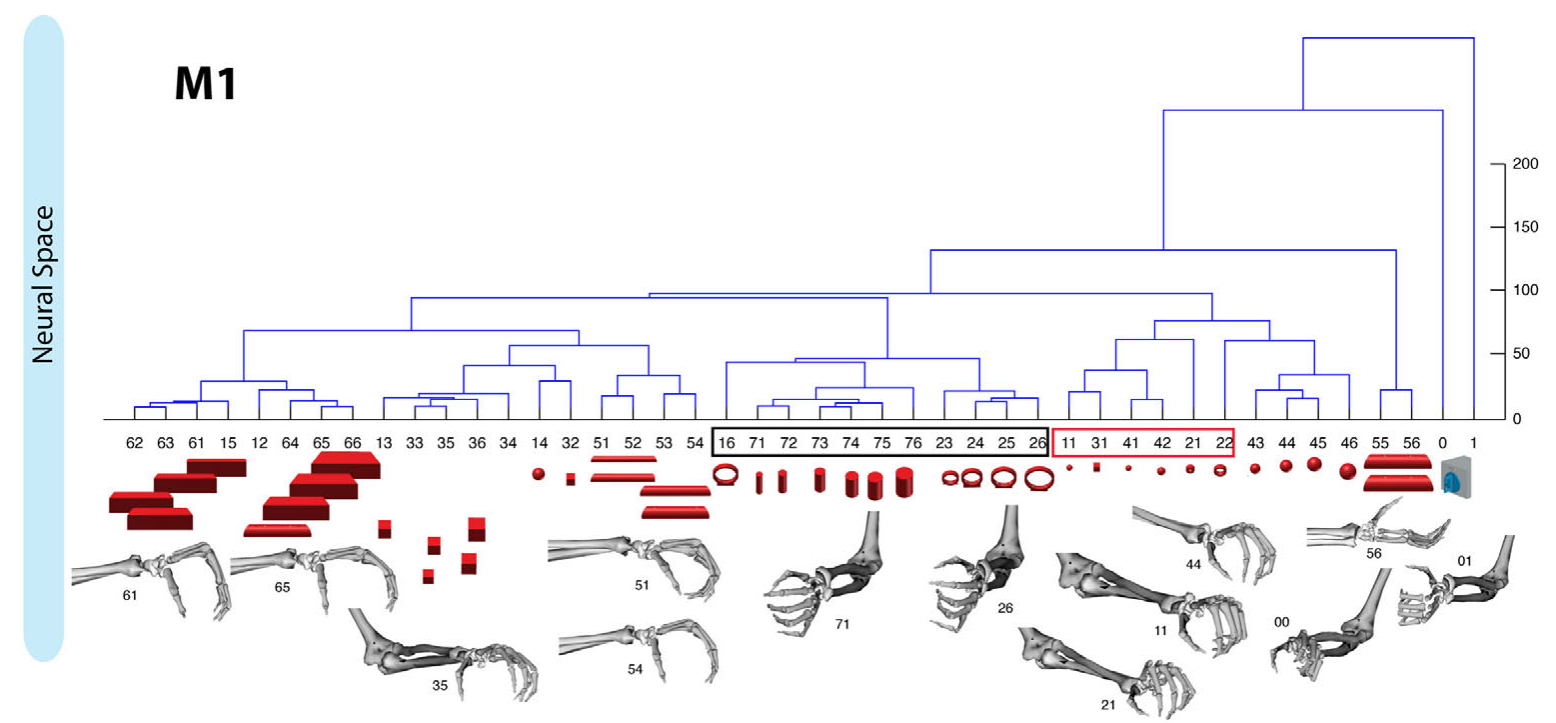

Supplemental figure 5. M1-Hierachical Cluster Analysis in animal Z. Dendrograms represent the complete M1 population during the plan epoch in animal $Z$. 



\subsection{Decoding a wide range of hand configurations from macaque motor, premotor, and parietal cortices}

Brain-machine-interfaces enable paralyzed patients to partially recover motor functions lost due to spinal cord injuries or diseases. In this chapter, a simple Bayesian classifier is presented that allowed decoding of a large number of hand configurations by analyzing neural activity recorded from macaque hand grasping areas AIP, F5, and M1. Furthermore, we successfully tested the possibility of translating the decoded grip types to an anthropomorphic robotic hand. These findings are highly relevant for the development of hand prosthetics that rely on control signals derived from higher cortical areas. The study is presented as published in the Journal of Neuroscience. 


\title{
Decoding a wide range of hand configurations from macaque motor, premotor, and parietal cortices
}

Neural decoding of hand configurations

\author{
Stefan Schaffelhofer ${ }^{1}$, Andres Agudelo-Toro ${ }^{1}$, and Hansjörg Scherberger ${ }^{1,2}$ \\ ${ }^{1}$ German Primate Center GmbH, D-37077 Göttingen, Germany \\ ${ }^{2}$ Deparment of Biology, University of Göttingen, D-37077 Göttingen, Germany
}

Correspondence should be addressed to Hansjörg Scherberger, Kellnerweg 4, D-37077 Göttingen, Germany. Email: hscherberger@dpz.eu

Acknowledgements: The authors thank $M$. Sartori for developing the musculoskeletal model in collaboration with our lab, F. Wörgötter for providing the robot hand, R. Ahlert, N. Nazarenus, and L. Burchardt for assistance in animal training, M. Dörge for technical assistance, L. Schaffelhofer for glove production, B. Lamplmair for providing illustrations, and J. Michaels and B. Dann, for their advice and helpful comments on an earlier version of the manuscript. This work was supported by the BMBF (BCCN II, DPZ 01GQ1005C). 


\section{Abstract}

Despite recent advances in decoding cortical activity for motor control, the development of hand prosthetics remains a major challenge. To reduce the complexity of such applications, higher cortical areas that also represent motor plans rather than just the individual movements might be advantageous. We investigated the decoding of many grip types using spiking activity from the anterior intraparietal (AIP), ventral premotor (F5), and primary motor cortex (M1). Two rhesus monkeys were trained to grasp 50 objects in a delayed task while hand kinematics and spiking activity from six implanted electrode arrays (total: 192 electrodes) were recorded. Offline, we determined 20 grip types from the kinematic data and decoded these hand configurations and the grasped objects with a simple Bayesian classifier. When decoding from AIP, F5, and M1 combined, the mean accuracy was $50 \%$ (using planning activity) and $62 \%$ (during motor execution) for predicting the 50 objects (chance level: $2 \%$ ), and substantially larger when predicting the 20 grip types (planning: $74 \%$, execution $86 \%$, chance level: $5 \%$ ). When decoding from individual arrays, objects and grip types could be predicted well during movement planning from AIP (medial array) and F5 (lateral array), whereas M1 predictions were poor. In contrast, predictions during movement execution were best from M1, whereas F5 performed only slightly worse. These results demonstrate for the first time that a large number of grip types can be decoded from higher cortical areas during movement preparation and execution, which could be relevant for future neuroprosthetic devices that decode motor plans.

Keywords: decoding; grasping; hand tracking; rhesus 


\section{Introduction}

Spinal cord injuries or motor diseases can lead to a disconnection of the spinal cord from the brain. Such paralyzed patients have reported that hand and arm functions are very important for them to recover (Anderson, 2004, Snoek et al., 2004). For these patients, myoelectric prosthetics are not applicable, since they depend on activated nerves in limbs or chest (Kuiken et al., 2009). In comparison, cortical neural interfaces can directly access brain activity and translate it into assistive control signals (Hatsopoulos and Donoghue, 2009, Scherberger, 2009). A better understanding of the cortical motor system together with improved decoding algorithms led to the development of brain interfaces for the control of computer cursors (Taylor et al., 2002, Ganguly and Carmena, 2009, Kim et al., 2011, Gilja et al., 2012) and robotic grippers (Hochberg et al., 2012, Collinger et al., 2013) that allow tetraplegic patients to regain physical interaction with their environment.

Despite these impressive advances, the neural guidance of hand prosthetics remains a major challenge. While reaching in space involves three degrees of freedom (DOF), this number increases to at least 23 DOFs when all joint angles of an anthropomorphic hand are considered. Controlling so many DOFs exclusively under visual feedback explains the difficulty of the neuroprosthetic substitution of hand function (Vargas-Irwin et al., 2010).

Alternatively, movement intentions can be decoded from higher-order planning signals of premotor and parietal cortex (Musallam et al., 2004, Townsend et al., 2011). Decoding higher-order motor plans (i.e., grip types) instead of many individual DOFs could help reduce the dimensionality problem for such decoding applications (Andersen et al., 2010). The ventral premotor cortex (specifically area F5) and the anterior intraparietal cortex (AIP) that show strong bidirectional anatomical connections (Luppino et al., 1999, Borra et al., 2008) are particularly well suited for this kind of task. Functionally, they are responsible for translating visual signals into hand grasping instructions. Neurons in both areas were identified to reflect visual information about the object being grasped (Murata et al., 1997, Murata et al., 2000) as well as the performed grip type (Baumann et al., 2009, Fluet et al., 2010). Compared to $M 1$, information in these areas is already accessible well before movement execution and has been used to decode largely different grip types such as power and precision grips (Carpaneto et al., 2011, Townsend et al., 2011). However, the question remains open whether detailed hand shapes could be differentiated from these areas as well.

In this study we demonstrate for the first time that fine differences in hand configurations can be decoded accurately from the cortical areas AIP and F5 during motor planning and execution. Furthermore, we compared the decoding capabilities of AIP and F5 with that of M1 and found major differences between them. Finally, the grip types selected from 27 DOF of the primate hand and arm 
could be translated to an anthropomorphic arm and hand of 16 DOF, hence demonstrating the possibility of converting high-level neural motor commands into neuroprosthetic robotic grips.

a

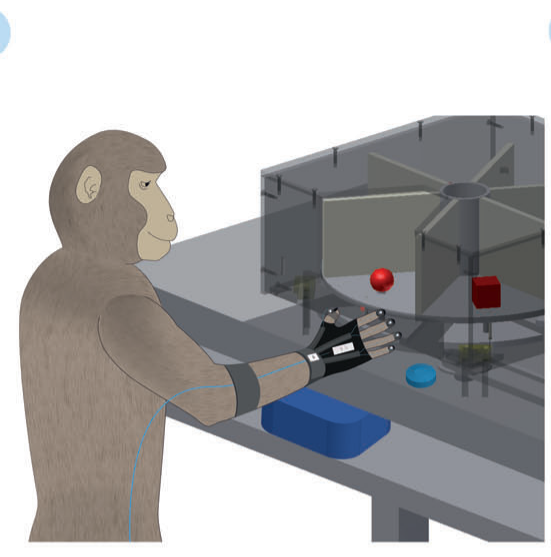

b

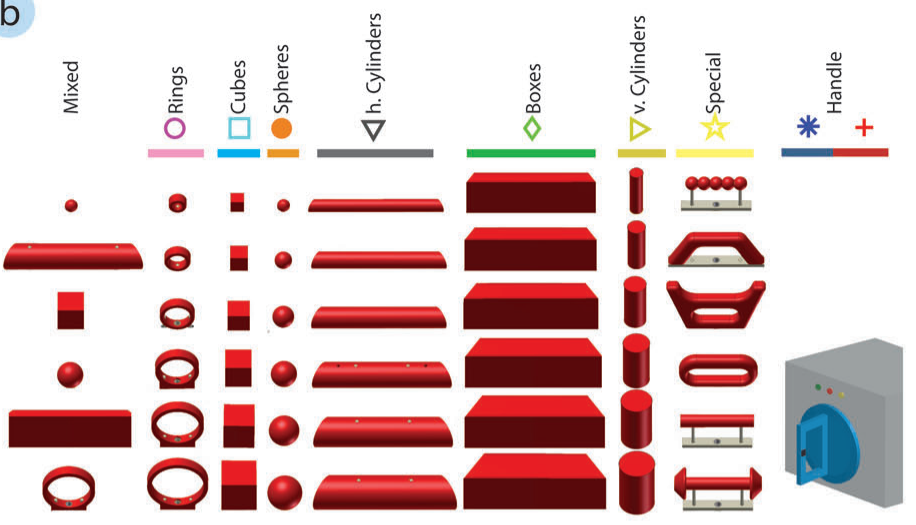

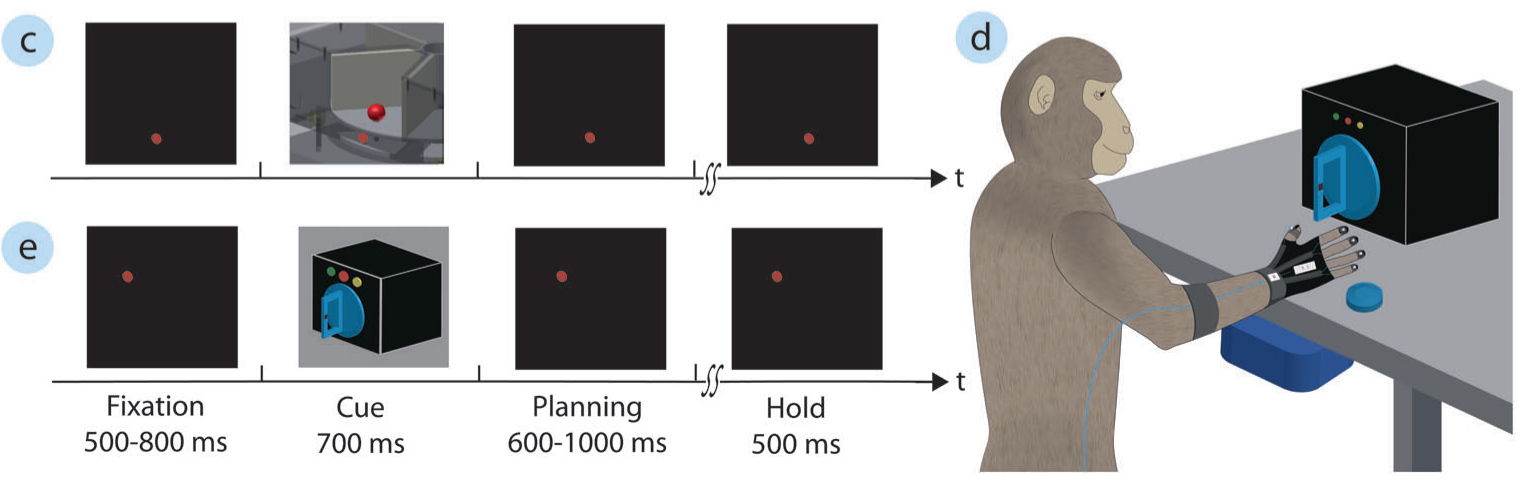

Figure 1. Experimental task. (a) Two macaque monkeys were trained to grasp a wide range of objects presented on a PC-controlled turntable. (b) In total, the animal grasped 48 objects mounted on 8 exchangeable turntables. (c) On each turntable, objects were presented in a pseudorandom order and were grasped within a delayed task consisting of eye-fixation, cue, planning, movement, and hold epochs. The monkeys performed the task in darkness, except during the cue epoch, when the objects were illuminated. (d) Within each recording session, monkeys also grasped a handle with two additional grips. (e) In this task, two supplementary LEDs instructed the animal to perform either a precision (yellow LED) or a power grip (green LED). 


\section{Materials and Methods}

\section{Basic procedures}

Two purpose-bread macaque monkeys (Macaca mulatta) participated in this study (Animal Z: female, $7.0 \mathrm{~kg}$; animal M: male, $10.5 \mathrm{~kg}$ ). They were first trained in a delayed grasping task to grasp a wide range of different objects while wearing a kinematic data glove (figure 1), then a head holder was implanted on the skull and electrode arrays were permanently inserted in the cortical areas AIP, F5, and M1. In subsequent recording sessions, neural activity and hand kinematics were simultaneously recorded while animals performed the grasping task. All analysis was performed offline. Animal care and all experimental procedures were conducted in accordance with German and European law and were in agreement with the Guidelines for the Care and Use of mammals in Neuroscience and Behavioral Research (National Research Council 2003).

\section{Experimental setup}

For behavioral training and experiments, the monkey sat in a customized primate chair with its head fixed. Graspable objects (handle or objects on a turntable) were presented in front of the animal at a distance of $25 \mathrm{~cm}$ at chest level (figure 1a). The setup design allowed a fast exchange of turntables in less than 1 minute and individual objects could be lifted vertically by $30 \mathrm{~mm}$. Custom-made software was used to control the turntable position and the pseudorandom sequence of object presentation. Object lifting and turntable position was monitored with a photoelectric barrier.

To obtain a high variation of grip types, we designed objects of different shape and size (figure $1 \mathrm{~b}$ ) including rings (outer diameter: $10 \mathrm{~mm}, 20 \mathrm{~mm}, 30 \mathrm{~mm}, 40 \mathrm{~mm}, 50 \mathrm{~mm}, 60 \mathrm{~mm}$ ), cubes (length: 15 $\mathrm{mm}, 20 \mathrm{~mm}, 25 \mathrm{~mm}, 30 \mathrm{~mm}, 35 \mathrm{~mm}, 40 \mathrm{~mm}$ ), spheres (diameters: $15 \mathrm{~mm}, 20 \mathrm{~mm}, 25 \mathrm{~mm}, 30 \mathrm{~mm}$, $35 \mathrm{~mm}, 40 \mathrm{~mm}$ ), cylinders (length: $170 \mathrm{~mm}$, diameter: $15 \mathrm{~mm}, 20 \mathrm{~mm}, 25 \mathrm{~mm}, 30 \mathrm{~mm}, 35 \mathrm{~mm}, 40$ $\mathrm{mm}$ ), and bars (length: $170 \mathrm{~mm}$, height $50 \mathrm{~mm}$, depth: $15 \mathrm{~mm}, 20 \mathrm{~mm}, 25 \mathrm{~mm}, 30 \mathrm{~mm}, 35 \mathrm{~mm}, 40$ $\mathrm{mm}$ ). Furthermore, a mixed turntable was used, holding mid-sized objects of various shapes (sphere $15 \mathrm{~mm}$, horizontal cylinder $30 \mathrm{~mm}$, cube $30 \mathrm{~mm}$, bar $10 \mathrm{~mm}$, ring $50 \mathrm{~mm}$ ), as well as a special turntable that contained objects of abstract forms (figure $1 \mathrm{~b}$ ). All objects had a uniform weight of 120 $\mathrm{g}$ independent of their size and shape.

In addition, power and precision grips were performed on a graspable handle (Baumann et al., 2009, Fluet et al., 2010). On the handle, two touch sensors were placed in small, clearly visible recessions to detect the contact of the animal's thumb and index finger during precision grips, whereas power grips were detected by an infrared light barrier at the inside of the handle and a pulling force sensor. 


\section{Behavioral paradigm}

Monkeys were trained in a delayed grasp and hold paradigm (figure 1c). While in complete darkness, an animal could initiate a trial by pressing a home button near its chest. Then, it had to fixate a red LED light while maintaining its hand on the home button. Fixating this red LED for a variable time (fixation epoch, duration: $500-800 \mathrm{~ms}$; mean, $650 \mathrm{~ms}$ ) turned on a spotlight that illuminated the graspable object (cue epoch, duration: $700 \mathrm{~ms}$ ). The spotlight was then turned off, but the animal had to withhold movement execution until the fixation LED blinked (planning epoch, 600-1000 ms; mean $800 \mathrm{~ms}$ ). Then the animal had to grasp and lift the object (movement-epoch) and hold it for $500 \mathrm{~ms}$ (hold epoch) in order to receive a liquid reward (small amount of juice). A following trial could then be initiated after an inter-trial interval of $1000 \mathrm{~ms}$. Error trials were immediately aborted without giving a reward. In case of the graspable handle (Fig 1.d), an additional yellow LED (or green LED) was turned on during the cue epoch to instruct the animal to perform a precision grip (or power grip) as shown in Figure 1e.

Objects were mounted on eight turntables in groups of six (see columns of figure 1b). During each block of trials, the objects of one turntable were presented in pseudorandom order until all objects were grasped successfully at least 10 times. Then, the turntable was exchanged and another block of trials started until all objects were tested. Finally, power and precision grip trials were performed with the graspable handle (10 trials pseudorandomly interleaved). To maintain a high motivation, animals were restricted from water access up to 24 hours prior to training or testing.

Eye movements were monitored with an infrared camera (ISCAN, Woburn, MA, USA) though a halfmirror. All behavioral and task relevant parameters, i.e., eye position, activation of buttons, and all stimulus presentations, were controlled using custom-written behavioral control software implemented in LabVIEW Realtime (National Instruments).

\section{Surgical procedures and imaging}

Before surgery, we performed a 3D anatomical MRI scan of the animal's skull and brain to locate anatomical landmarks (Townsend et al., 2011). For this, the animal was sedated (10 mg/kg ketamine and $0.5 \mathrm{mg} / \mathrm{kg}$ xylazine, i.m.), placed in the scanner (GE Signa HD or Siemens TrioTim; 1.5 Tesla) in a prone position, and T1-weighted images were acquired (iso-voxel size: $0.7 \mathrm{~mm}^{3}$ ).

Then in an initial procedure, a head post (titanium cylinder; diameter, $18 \mathrm{~mm}$ ) was implanted ontop of the skull (approx. stereotaxic position: midline, $40 \mathrm{~mm}$ ant., 20 deg forward tilted) and secured with bone cement (Refobacin Plus, BioMed, Berlin) and orthopedic bone screws (Synthes, Switzerland). After recovery from this procedure and subsequent training with head fixation, each animal was implanted in a second procedure with six floating microelectrode arrays (FMAs; 
MicroProbes for Life Science, Gaithersburg, MD, USA). Specifically, two FMAs were inserted in each area AIP, F5, and M1 (see figure 2). FMAs consisted of 32 non-moveable monopolar platinum-iridium electrodes (impedance: $300-600 \mathrm{k} \Omega$ at $1 \mathrm{kHz}$ ) as well as two ground and two reference electrodes per array (impedance $<10 \mathrm{k} \Omega$ ). Electrode length ranged between 1.5 and $7.1 \mathrm{~mm}$ and were configured as in Townsend et al. (2011).

Electrode array locations are depicted in figure 2c-d. In both animals the lateral array in AIP (AIPlat) was located at the end of the intraparietal sulcus at level of PF, whereas the medial array (AIPmed) was placed more posteriorly and medially at the level of PFG (Borra et al., 2008). In area F5, the lateral array (F5lat) was positioned approximately in area F5a (Belmalih et al., 2009, Borra et al., 2010), whereas the medial array (F5med) was located in F5p in animal $Z$ and at the border of F5a and F5p in animal M. Finally, both arrays in M1 (M1lat, M1med) were positioned in the hand area of M1 (anterior bank of the central sulcus at the level of the spur of the arcuate sulcus and medial to it) (Rathelot and Strick, 2009).

All surgical procedures were performed under aseptical conditions and general anesthesia (e.g., induction with $10 \mathrm{mg} / \mathrm{kg}$ ketamine, i.m., and $0.05 \mathrm{mg} / \mathrm{kg}$ atropine, s.c., followed by intubation, $1-2 \%$ isofluorane, and analgesia with $0.01 \mathrm{mg} / \mathrm{kg}$ buprenorphene, s.c.). Heart and respiration rate, electrocardiogram, oxygen saturation, and body temperature were monitored continuously. Systemic antibiotics and analgesics were administered for several days after each surgery. To prevent brain swelling while the dura was open, the animal was mildly hyperventilated (end-tidal $\mathrm{CO}_{2}<30$ $\mathrm{mmHg}$ ) and mannitol kept at hand. Animals were allowed to recover for at least 2 weeks before behavioral training or recording experiments recommenced.

\section{Hand kinematics}

To record the kinematics of the monkey's hand and arm, we have developed an instrumented glove for small primates, as previously described (Schaffelhofer and Scherberger, 2012). This kinematic tracking device is based on an electro-magnetic tracking system (WAVE, Northern Digital) and consists of seven sensor coils that are placed on all fingertips, the back of the hand, and at the lower forearm just proximal to the wrist (figure 1a). For calibration purposes, an additional sensor was also temporally placed on top of each metacarpal phalangeal joint (MCP). Using this instrumented glove, the dynamic 3D position of the distal interphalangeal joint (DIP), the proximal interphalangeal joint (PIP), and the MCP position of all fingers were determined as well as the 3D position and orientation of the hand. Furthermore, the wrist sensor provided the orientation of the forearm and hence the 3D position of the elbow. Since the monkey was head-fixed, the shoulder position could be assumed constant. This provided a full kinematic description of the arm and hand (including 18 joints and 27 
degrees of freedom (DOF)) with a temporal resolution of $100 \mathrm{~Hz}$. Data acquisition, processing and visualization were realized in a custom-made Graphical User Interface in MATLAB (The MathWorks, Inc., MA, USA).

Electromagnetic sensors could be tracked even when visually occluded, since they did not depend on line of sight to a camera. However, they were influenced by the presence of inductive metals (Raab et al., 1979). Therefore, ferromagnetic materials had to be largely avoided in the setup, including the turntable, all graspable objects, and the primate chair.

\section{Neural recordings}

From the implanted electrode arrays, we record spiking activity (single units and multiunits) simultaneously from a total of 192 electrodes in AIP, F5, and M1 (Fig 2). Neural activity was sampled with at a rate of $24 \mathrm{kHz}$ with a resolution of $16 \mathrm{bit}$ and stored to disk together with behavioral data and hand and arm kinematics using a RZ2 Biosignal Processor (Tucker Davis Technologies, FL, USA).

a

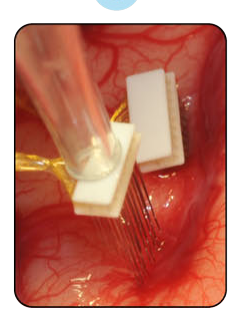

b

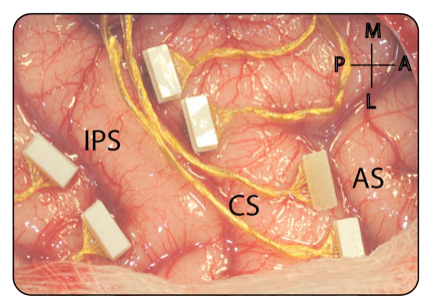

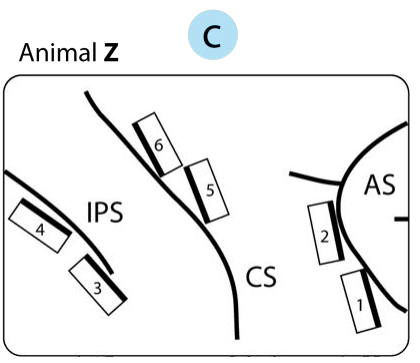

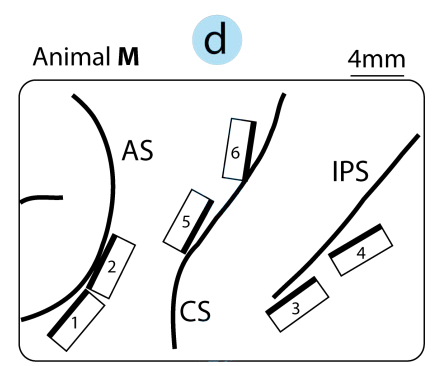

Figure 2. Electrode array implantation. (a-b) Animals were implanted with multiple floating micro-electrode arrays (FMAs) in area AIP, F5, and M1. (a) Each array consisted of 32 individual electrodes of variable length (1.5-7.1 $\mathrm{mm}$ ) and were (b) placed in the bank of the sulcus. In animal $Z(b-c)$ and animal $M(d)$, two arrays were implanted in each area: at the lateral end of the intraparietal sulcus (IPS) in AIP, in the posterior bank of the arcuate sulcus (AS) in area F5, and in the anterior bank of the central sulcus (CS) in the hand area of M1. (c-d) Schematics of FMA placements also show the FMA numbering for animal Z (right hemisphere) and animal M (left hemisphere), respectively. The dark edge of each FMA indicates the row of longest electrodes (max. $7.1 \mathrm{~mm}$ ). Annotations as in $\boldsymbol{b}$. In this study, individual arrays are labeled as F5/at (\#1), F5med (\#2), AlPlat (\#3), AlPmed (\#4), M1lat (\#5), and M1med (\#6). Scale: long edge of FMA is $4 \mathrm{~mm}$. A, anterior; L, lateral; M, medial; $P$, posterior. 


\section{Data analysis}

\section{Hand kinematics.}

The trajectories of all 18 joints of the moving hand and arm as well as of the fingertips were used to drive a musculoskeletal model (figure 6a) that was scaled to match the primate-specific anatomy (Schaffelhofer et al., 2014). The model was implemented in OpenSim (Delp et al., 2007) and allowed extracting all hand and arm joint angle positions, including: flexion/extension (MCP, PIP, DIP) and adduction/abduction (MCP) of all fingers, wrist flexion/extension, adduction/abduction, and pronation/supination, as well as elbow flexion and shoulder elevation, rotation, and adduction/abduction (27 DOF in total).

\section{Spike sorting}

All spike sorting relevant for analysis was performed offline. First, we applied WaveClus (Quiroga et al., 2004) for automatic sorting and subsequently the OfflineSorter (Plexon TX, USA) for manual resorting. This procedure provided an objective and automatized classification of neurons and an additional evaluation of cluster quality with respect to signal stability (e.g., drift) and interspike interval histograms.

\section{Single unit and population activity}

Firing rate histograms (FRH) were created to present tuning attributes of example neurons from AIP, F5 and M1 (figure 3a, d, g). For this, the spike rates were visualized by replacing each spike with a Gaussian kernel function ( $\sigma=50 \mathrm{~ms}$ ) that were then averaged across all spikes and trials (Baumann 2009).

Furthermore, we computed the cross-modulation-depth of individual neurons (figure $3 b, e, h$ ). The modulation depth (MD) between two conditions (e.g., $x$ and $y$ ) was defined as the absolute difference of the averaged firing rate (across all $\mathrm{n}$ trials) of the neural activity $f$ between condition $\mathrm{x}$ and y:

$$
m d(x, y)=\frac{1}{n}\left|\sum_{i=1}^{n}\left(f_{x}^{i}-f_{y}^{i}\right)\right|
$$

The MD between all condition pairs was computed and the resulting matrix was plotted as a color map for individual task epochs. To obtain information about the significance of the modulation depth of individual condition pairs, we performed a multi-comparison test across all task conditions (ANOVA and post-hoc Tukey-Kramer criterion, $\mathrm{p}<0.01$; Matlab functions: anova1, multcompare) 
(figure 3c, $\mathrm{f}$, i). Furthermore, we defined the coefficient of separability (CS) for each neuron and task epoch as the fraction of significant condition pairs with respect to all pairs.

Finally, the large number of conditions allowed comparing the encoding properties of individual neurons between different task epochs. For this, we computed the Pearson correlation coefficient between the modulation depth maps.

For visualizing the population activity during the task, we computed for each neuron a sliding ANOVA ( $p=0.01$; time steps: $20 \mathrm{~ms}$ ) across the 6 conditions of the mixed turntable. The fraction of significantly modulated neurons at each time step was then calculated separately for each area and recording array (see figure 4).

\section{Decoding}

For decoding, our goal was to predict the presented object or the intended grip type from the recorded neuronal activity as accurate as possible. For this the decoding classes (or categories) were defined as the presented objects ( 50 classes) or the grips used for grasping these objects, ( 20 classes; see below, Grip-type classification). For each decoding procedure only simultaneously recorded spiking activity from single and multiunits were included. This way, a real-time decoding could be simulated as closely as possible. The mean firing rate of all single units and multiunits were computed for the specific task epochs and used as the input parameters for the classifier.

We used a naive Bayesian classifier for decoding that has been shown to reach close to optimal performance within a large family of classifiers for this kind of data (Scherberger et al., 2005, Subasi et al., 2010, Townsend et al., 2011). (Naively) assuming statistical independence between the firing rates $f_{\mathrm{i}}$ of different neurons $(i=1, \ldots, n)$, the likelihood function $L(c)$ can be computed as $L(c)=$ $\prod_{i=1}^{N} p\left(c \mid f_{i}\right)$, where $p\left(c \mid f_{i}\right)$ denotes the probability of observing condition $c$ for a given firing rate $f_{i}$ of neuron $i$. Using Bayes equation

$$
p\left(c \mid f_{i}\right)=\frac{p\left(f_{i} \mid c\right) \cdot p(c)}{p\left(f_{i}\right)}
$$

this probability can be expressed with $p\left(f_{i} / c\right)$, which denotes the probability of observing the firing rate $f_{i}$, given condition $c$. Furthermore, the uniformly distributed term $p(c)$ and the term $p\left(f_{i}\right)$, which is independent of $c$, can be summed as $k_{i}$, which reduces the equation to $p\left(c \mid f_{i}\right)=k_{i} \cdot p\left(f_{i} \mid c\right)$. Because the factor $k_{i}$ is constant across conditions, the likelihood function can be further reduced to

$$
L(c)=\prod_{i=1}^{N} p\left(f_{i} \mid c\right) .
$$


The condition showing the highest likelihood for the observed firing rates was then selected as the decoded condition:

$$
\hat{c}=\operatorname{argmax}_{c}(L(c))
$$

To train the decoder, the probability distributions $p\left(f_{i} \mid c\right)$, which were estimated from the mean firing rates observed in the training data under the assumption of a the Poisson distribution, had to be determined for each condition. For testing decoding performance, we applied a leave-one out cross-validation, which ensured that data sets used for training were not used for testing.

\section{Neuron-drop analysis}

To measure the decoding accuracy as a function of neurons used for decoding, we performed a neuron-dropping analysis. This simple algorithm starts by training the decoder with a randomly selected neuron. Then, the number of included cells was increased in steps of 1 until all available neurons were included to the decoder. For each increment (step), the random selection of cells was repeated 100 times. The neuron-drop analysis was applied to each microelectrode array and allowed a direct and objective comparison between cortical areas and sub-areas. Paired t-tests $(p<0.01)$ were applied for statistical comparison between areas and task epochs.

\section{Grip-type classification}

Electrophysiological studies in the macaque hand areas AIP and F5 revealed not only motor discharges, but also responses to the visual representation of objects, and it was assumed that such visual cells code attributes of objects such as shape, size, and/or orientation (Murata et al., 1997, Murata et al., 2000). To demonstrate that hand configurations (i.e., hand shape) can be decoded independently from such object information, we classified the performed trials based on the grips applied to the many objects. This re-grouping of trials according to grip type allowed an objectindependent decoding, and furthermore could help reduce redundancies among different objects, e.g. objects of different shape that were grasped by the same grip.

For the classification of grip types, we recorded finger, hand, and arm kinematics in each recording session simultaneously with cortical recordings. The hold epoch revealed the highest variation of grip type under the most stable kinematic conditions. We therefore selected the hold epoch to extract joint angles for grip type classification.

To find the similarities or differences in the classification of grip types across performed trials and kinematic dimensions (i.e., >500 trials and 27 DOF) we computed the Euclidean distance between each pair of trials (Matlab function: pdist). Based on this distance measure, a hierarchical cluster tree was created that described the proximity of trials to each other (Matlab function: linkage, criterion: 
"ward"). As a final step, we were searching for natural groupings within the dataset (number of clusters). Due to the large number of trials and objects used, the kinematic space represented a natural and non-discrete distribution of hand configurations. As a result, the dataset did not reveal an optimal number of clusters that showed a maximum separation (silhouette test). Therefore, we set the number of clusters heuristically to a value of 20 , hence demonstrating a good compromise between quantity of grip types and quality of kinematic separability (e.g. see Fig 6d). Furthermore, the constant number of clusters across multiple recordings allowed a more objective comparison between the decoding results of sessions and animals.

\section{Offline robotic control}

To illustrate the possible translation of the primate arm and hand model with its 27 DOF in a lowerdimensional robot arm and hand (here: 16 DOF), we employed a 7-DOF robot arm (Barrett Technology WAM $^{\text {TM }}$ Arm, Newton, MA, USA) and a 5-fingered robotic hand (SCHUNK GmbH, Lauffen, Germany). To translate the primate model on the robot arm and hand, we solved the inverse kinematic problem for the arm and employed linear transforms for the fingers. Although the robot arm had equal DOF as the primate arm, its rotation axes (3 DOF for shoulder, 3 DOF for wrist, and 1 DOF for elbow) differed from the primate model. We solved this inverse kinematic problem (Paul, 1982) by matching the robot posture to the primate upper arm orientation with respect to the shoulder and to the primate hand orientation with respect to the forearm.

The five-fingered robotic hand had 9 actuated DOF: (1) thumb abduction, (2) combined carpometacarpal joint (CMC), MCP, and DIP flexion of thumb, (3) index MCP flexion, (4) combined PIP and DIP flexion of index finger (5) middle MCP flexion, (6) combined PIP and DIP flexion of middle finger, (7) combined MCP, PIP, DIP flexion of ring finger, (8) combined MCP, PIP, DIP flexion of little finger, (9) combined spread of digits. Each actuated DOF of the robot hand was linearly coupled to the corresponding DOF of the primate hand, such that the movement range of the primate DOF was linearly mapped on the robotic movement range.

\section{Results}

The data in this study present in total 20 recording sessions from two macaque monkeys (10 recordings per monkey). Both animals were implanted with six 32-channel FMAs in the handgrasping areas AIP, F5, and M1 (two FMAs per area; 192 channels in total). This facilitated simultaneous recording from $355 \pm 20$ and 202 \pm 7 (mean \pm SD) single units and multiunits in monkeys $M$ and $Z$, respectively. All implanted arrays were functional and allowed recording of stable neuronal populations. Across areas, the acquired single units and multiunits were distributed as follows: $25.2 \%$ 
for AIP, 32.3\% for F5, and $42.5 \%$ for M1 in animal M; and 29.2\% for AIP, 37.3\% for F5, and $33.5 \%$ for M1 in animal $Z$.

Simultaneous to the neural recordings, we tracked finger, hand, and arm movements across all recording sessions using an instrumented glove (Schaffelhofer and Scherberger, 2012) and fit a musculoskeletal model of the primate hand and arm that consisted of 27 DOF at 18 joints (Schaffelhofer et al., 2014). From these movement kinematics, we then classified and decoded a wide range of hand configurations that the animal applied to grasp the 50 heterogeneous objects of our task.

\section{Neuron tuning properties}

Neurons recorded in this study presented attributes consistent with previous studies of area AIP (Murata et al., 2000, Baumann et al., 2009), F5 (Rizzolatti et al., 1988, Raos et al., 2006, Fluet et al., 2010), and the hand area of M1 (Schieber, 1991, Schieber and Hibbard, 1993, Vargas-Irwin et al., 2010).

In AIP, neurons showed a strong response and tuning during the cue epoch of the task, when the objects were illuminated. These attributes are illustrated by the example neuron in figure $3 \mathrm{a}$. It demonstrated a rapid increase in firing rate after cue presentation and a high selectivity for grasping conditions (i.e., for specific objects). Visually presenting the horizontal cylinders and the bar objects resulted in the highest response. These object groups shared similar visual attributes (i.e., being long and horizontal) but also required similar kind of grips (enclosure of big objects with flexed digits and wide aperture between index finger and thumb). Figure $3 b$ visualizes the relative differences in firing rate (i.e., the modulation depth) between all pairs of conditions (i.e., objects) in the considered task epochs (see Methods section). For this neuron, modulation depth was maximal $(34.4 \mathrm{~Hz})$ in the cue epoch. Neural tuning was consistent throughout the task, as indicated by the high correlation coefficient between the modulation depth matrix of the cue and the hold epoch ( $c=0.92$ ). Furthermore we performed a multicomparision analysis within each task epoch to identify those task condition (object) pairs, for which the neural firing rate was significantly different (Tukey-Kramer criterion, $\mathrm{p}<0.01$, see Methods). Pairs of conditions with significant differences are shown in red in figure 3 c. Overall, $57.5 \%$ and $51.83 \%$ of all condition pairs had significantly different firing rates in the cue and planning epoch, respectively (coefficient of separability, CS, see Methods), thus demonstrating a high object selectivity of this AIP neuron.

Figure 3d illustrates an example neuron of F5. It was activated during cue presentation (CS: $33.95 \%$ ), reached its maximum selectivity (figure $3 \mathrm{f}$ ) in the planning epoch (CS: $42.9 \%$ ), and was followed by a 
strong response in the movement epoch (CS: 42.2\%). Maximum modulation depth was observed during movement execution $(39.8 \mathrm{~Hz}$, see figure $3 \mathrm{f})$. Furthermore, not only the coefficient of separability was similar during motor preparation and execution, but also neural tuning, as demonstrated by the high correlation between the modulation depth maps of the planning and movement epoch $(c=0.76)$.

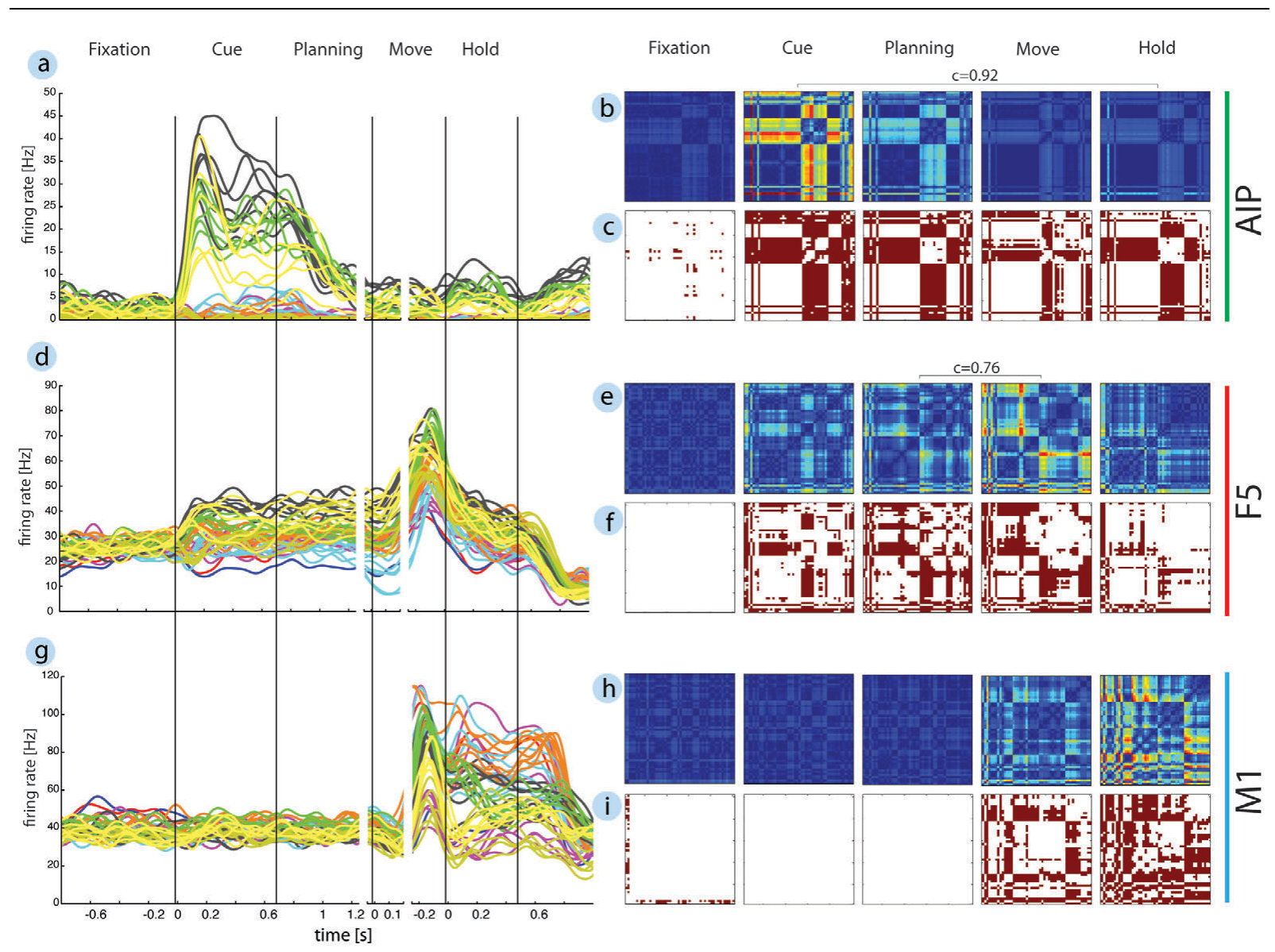

Figure 3. Neural coding of grasping actions. $(\boldsymbol{a}, \boldsymbol{d}, \boldsymbol{g})$ Firing rate histograms (FRH) are shown for three simultaneously recorded example neurons from area $A I P, F 5$, and $M 1$, respectively. Each line represents the average firing rate for a specific grasping condition (i.e., 50 objects) vs. time. The color code matches the object shape as in figure $1 b$. (b,e,h) Crossmodulation-depth plots (CMD) reflect the relative difference in firing rate between all pairs of grasping conditions $(50 \times 50$ pairs) for all 5 epochs. Firing rates were normalized relative to the maximum modulation depth found across all epochs. Pixels towards red represent pairs with maximum modulation depth, whereas pixels towards blue represent pairs without difference in firing rate. (c,f,i) Furthermore, a multi-comparison analysis (MC) revealed significant differences (in red) between condition pairs. The order of columns/rows for CMD- and MC plots is the same as in figure 5a. (a-c) The AIP neuron showed the highest modulation depth during the cue epoch and an additional bump during tfirihe hold epoch. (d-f) The example F5 neuron demonstrated a high modulation depth in the planning epoch and a further increase during motor execution. (g-i) The M1 motor neuron showed no significant coding during motor preparation (i.e., cue and planning), but became highly active during motor execution (i.e., movement and hold). Horizontal brackets indicate significant correlation coefficient c of MD maps between epochs. 
Not surprisingly, M1 neurons demonstrated the strongest response during movement execution (figure $3 \mathrm{~g}$ ). None of the condition pairs were significantly tuned before or during the preparation epoch (figure 3h). However, in the movement and hold epoch, when the monkey grasped and held the objects, $38.2 \%$ and $54.8 \%$ of the condition pairs were significantly different (CS, Fig. 3i). The illustrated $\mathrm{M} 1$ neuron showed a maximum modulation depth of $72.3 \mathrm{~Hz}$ during the hold epoch (figure $3 h)$.

All example neurons showed a high selectivity during motor execution, especially neurons recorded from M1 and F5. They demonstrated a surprisingly high differentiation between a wide range of grip type conditions. In AIP and F5, these attributes were also represented during the motor preparation epochs (move and hold). The multi-comparison analysis and the correlation of modulation depth matrices between planning and motor epochs highlight that these neurons represent movement well before execution, which makes them potentially suitable for the decoding of intended hand configurations, i.e., well before movement execution.

Individual neurons could demonstrate tuning already in the fixation epoch. This effect is explained by the block-wise task design required for presenting the large number of conditions (i.e., grips on handle and individual turntables). In figure $3 g$, the example neuron showed an increased firing rate when the handle was mounted in front of the animal, therefore the presented cell could differentiate between the handle and the turntable task (figure 3i). However, none of the neurons showed significant tuning in the fixation epoch within the group of the handle or the turntables, which demonstrates the non-predictability of individual conditions within each block of trials.

The attributes of single units could be confirmed at the population level (figure 4). Similar to the example cell in AIP, the population of AIP units showed a strong response to the presentation of objects. However, we found important differences between the lateral (AIPlat) and the medial array (AIPmed). Whereas AIPmed showed its strongest response in the cue epoch with a fraction of $42 \%$ (monkey Z) and $30 \%$ of significantly tuned units (monkey M), the population from AIPlat had a peak activation during motor execution with a fraction of $36 \%$ (monkey $\mathrm{Z}$ ) and $23 \%$ of tuned units (monkey $M$, sliding window ANOVA; $p<0.01$ ). These consistent results from both animals suggest a more visual role of AIPmed, whereas AIPlat might be rather motor-related (figure 4).

Similar to AIP, we also found substantial differences between the medial and lateral arrays of F5. In both animals, F5lat showed a higher fraction of tuned units during the planning epoch than the F5med population. However, in contrast to AIPmed that showed its strongest contribution during the cue epoch, F5lat demonstrated an additional increase of tuned units during movement execution. Although F5med had a weaker planning activity than F5lat, its contribution to our task was essential, 
as indicated by the fraction of $51 \%$ (animal Z) and $32 \%$ of tuned cells (animal M) during movement execution.

In $M 1$, the main population response occurred during the movement epoch with a fraction of $78 \%$ of tuned units in M1med of monkey $Z$ and of $69 \%$ in M1lat of monkey $M$, whereas only a small fraction of units represented planning activity. Also, M1 motor responses showed their peak activity aligned to the beginning of the hold epoch, which further supports the important role of M1 for hand movement generation. Together, the F5 and AIP populations both showed strong planning activity at the single unit level, which underscores the potential significance of these areas for decoding applications.
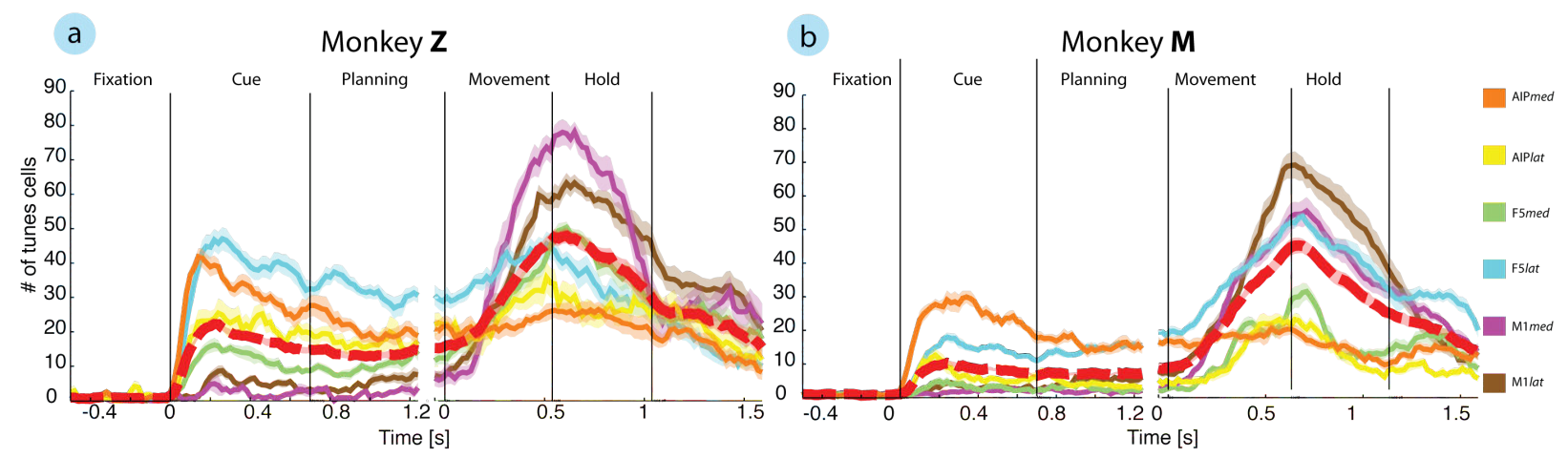

Figure 4. Population activity. Individual curves describe the percentage of tuned units vs. time separately for (a) animal Z and (b) animal M for recording arrays AIPmed, AIPlat, F5med, F5lat, M1med, and M1lat. The color-code for each array is consistent throughout the manuscript.

\section{Object based decoding}

Previous studies have investigated higher cortical regions such as area AIP and/or F5 to decode grip types before movement execution (Subasi et al., 2010, Carpaneto et al., 2011, Townsend et al., 2011, Carpaneto et al., 2012). These studies, however, focused exclusively on large differences in hand configurations such as precision and power grips applied to handles of different orientations (up to 10 conditions) or when grasping objects of highly different shape. In contrast, here we investigated the possibility of decoding fine differences of grips performed on a large number of objects. In total, the monkeys grasped $\sim 50$ objects that caused a high variability of hand shapes. Note that small differences in object size - while sharing the same object shape - elicited fractional difference in hand shape (see section: Grip type based decoding). Similar to previous studies (Baumann et al., 2009, Fluet et al., 2010, Townsend et al., 2011, Lehmann and Scherberger, 2013), we focused on the planning and hold epoch that were performed in darkness. This way, visual responses were avoided, while preparatory and motor signals became disambiguated. 

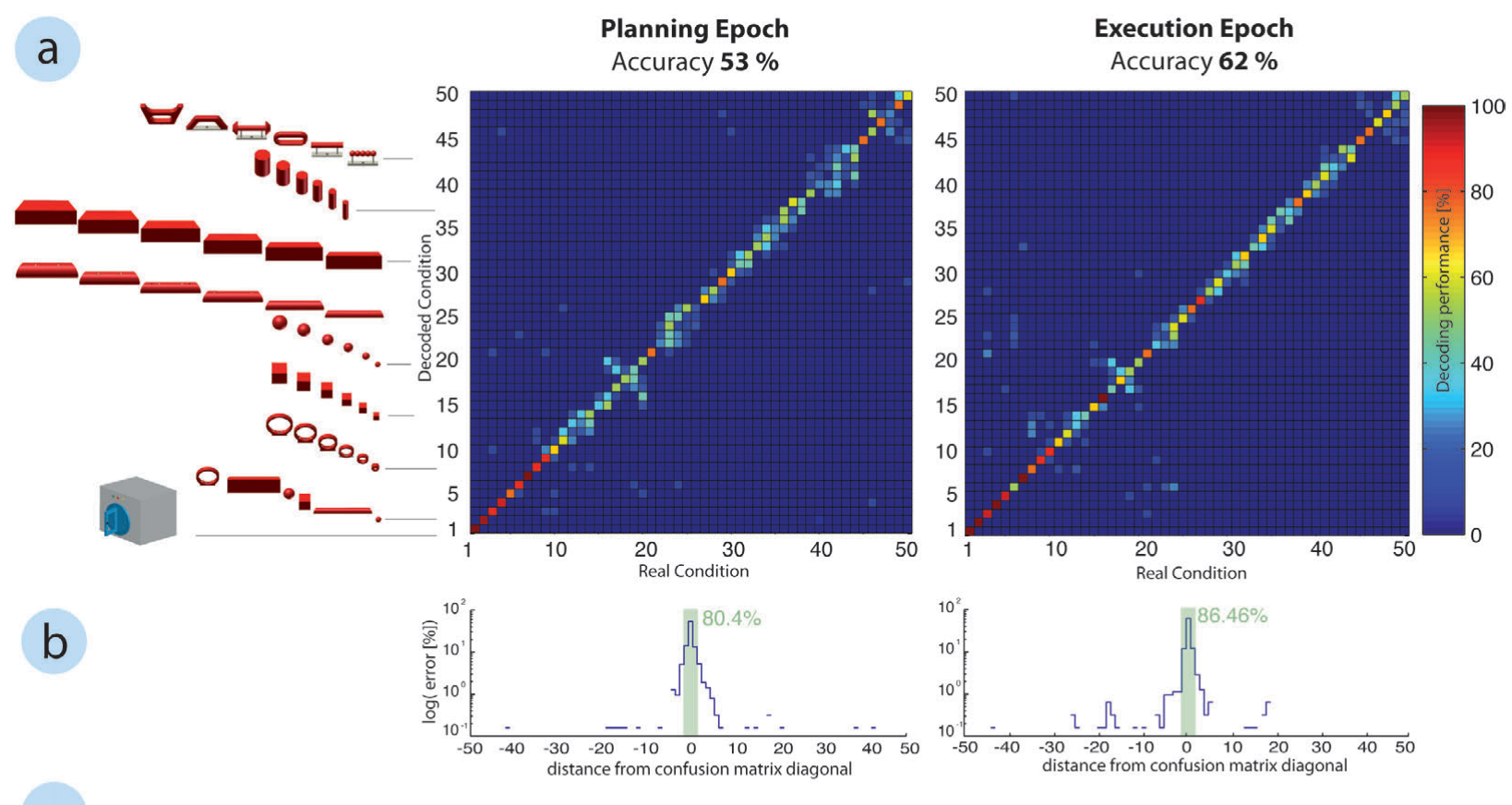

C
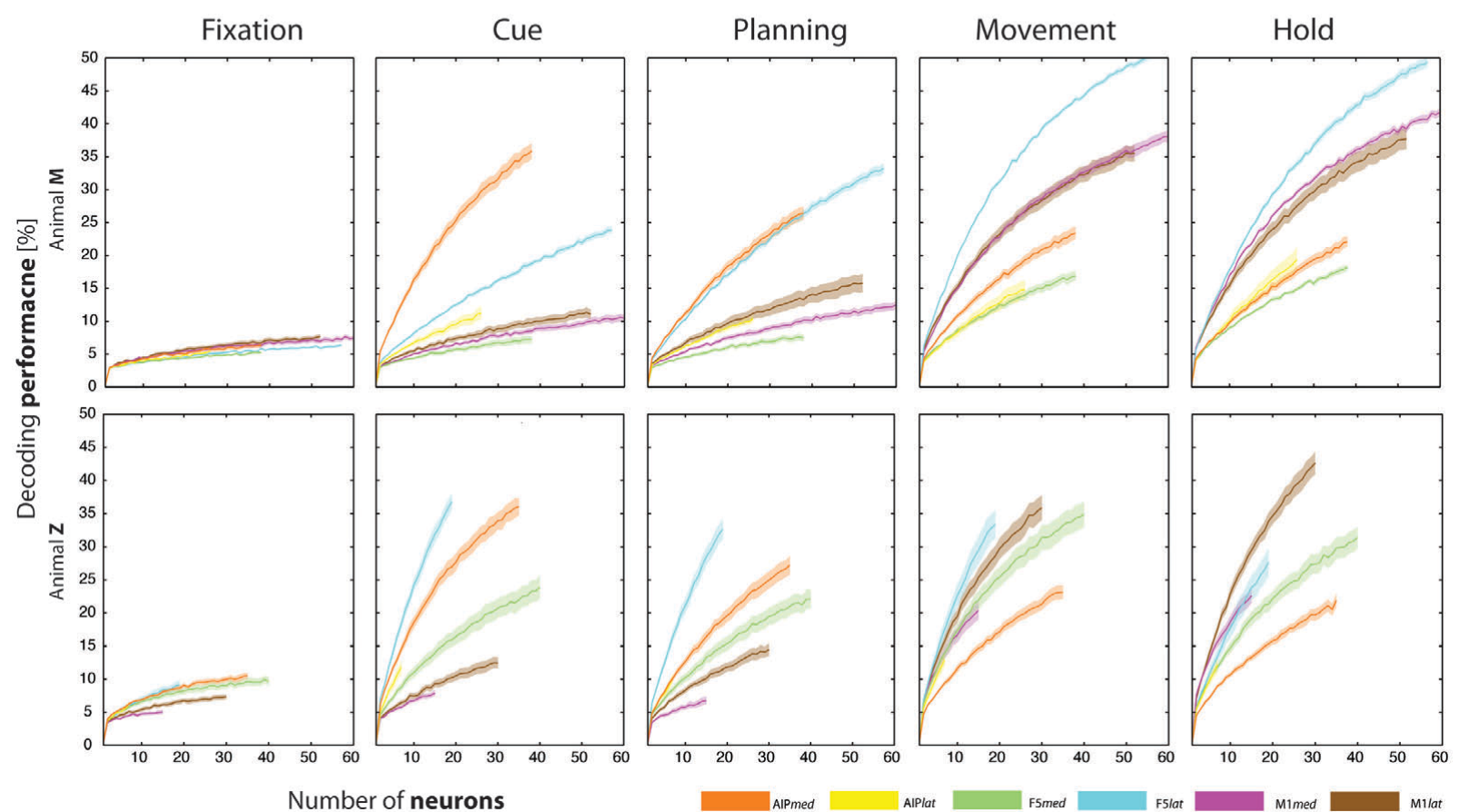

Figure 5. Object-based decoding. (a) The confusion matrix shows the decoding results from the planning and the hold epoch of a single recording session. Each decoding condition is illustrated by the 3D object grasped by the monkey. From 1 to 50: 12 precision and power grip on handle, 3-8 mixed turntable, 9-14 rings, 15-20 cubes, 21-26 balls, 27-32 horizontal cylinders, 33-38 bars, 39-44 vertical cylinders, 45-50 abstract shapes. (b) Error distribution in the confusion matrix as a function of distance to the matrix diagonal; note the logarithmic scale for the planning and the motor epoch. Green bar sums the percentage of trials with correct and distance-1 errors. (c) Neuron-drop analysis for all task epochs and both animals across all recording sessions. Decoding performance is plotted vs. the number of randomly selected neurons for each of the implanted microelectrode arrays for each epoch and animal. Solid lines show the mean decoding performance for specific arrays, whereas shades indicate standard error of the mean across 10 sessions in each animal. Lines stop at the minimal number of recorded neurons across all sessions. 
Decoding results of one example session are presented in figure 5. Using maximum likelihood decoding with cross-validation (see Materials and Methods), we found a high correlation between the real conditions and the decoded conditions in both the planning and the hold epoch, as illustrated in the confusion matrices (figure 5a). Error trials did not spread across all conditions but were most likely attributed to neighboring condition classes (e.g., cylinders of $30 \mathrm{~mm}$ in diameter could be confused with cylinders of $35 \mathrm{~mm}$ diameter). Objects were arranged in the matrix according to their similarity (shape and size). These effects were further visualized in figure $5 b$, where success and error rates were plotted on a logarithmic scale against distance from the confusion matrix diagonal. For the recording session displayed in figure $5 a, 53.0 \%$ and $62.4 \%$ of all trials were correctly assigned during the planning and hold epoch, respectively. However, the majority of error trials (58\%, 64\% for planning and hold respectively) were assigned to a class that was neighboring the correct (true) class. To evaluate the total error distribution, we additionally averaged the confusion matrices across all sessions from both animals. In this population, the majority of trials were correctly decoded with $51.3 \%$ and $60.7 \%$ during the planning and hold epoch, respectively, similar to the presented example session (Fig. 5a). Again, most of the errors were assigned to an adjacent class. Of all errors, 59\% (planning) and 62\% (hold) were incorrectly classified to a neighboring class. Allowing the assignment to such an adjacent class would therefore boost the decoding performance to $80.2 \%$ and $85.2 \%$ (planning and hold, respectively), while chance level would increase only to $6 \%$ (3 out of 50 conditions). These 'relaxed' accuracies are highlighted for the example session in figure $5 \mathrm{~b}$ (green bars).

Across the entire dataset of 10 decoding sessions per animal, the 50 object conditions could be decoded from the planning epoch with an accuracy of $48.7 \pm 3.6 \%$ and $51.9 \pm 3.4 \%$ (mean \pm s.d.) in animal $M$ and $Z$, respectively. This performance was $23.9 x$ and $26 x$ above chance (2\%). During motor execution (i.e., from the hold epoch) the average decoding accuracy was even larger: $62.9 \pm 3.6 \%$ and $61.4 \pm 4.1 \%$ (monkey $M$ and $Z$, respectively), corresponding to $31.5 x$ and $30.7 x$ above chance (2\%). This means, that decoding accuracy in the hold epoch was on average 14.2 and 9.5 percentage points higher than in the planning epoch (animal $M$ and $Z$, respectively). This improvement was significant $(p<0.001,2$-way ANOVA) in both animals.

Furthermore, we explored the functional differences of the various cortical areas and recording sites separately in each electrode array: (1) F5lat, (2) F5med, (3) AIPlat, (4) AIPmed, (5) M1lat, and (6) M1med (array numbering as in figure 2). To make the analysis fair, we applied a "neuron-drop" procedure that evaluated the decoding performance as a function of the number of randomly selected neurons included in the analysis (figure $5 \mathrm{c}$ ). This analysis allowed the following key observations: in the motor preparation epochs (i.e., cue and planning epochs), AIPmed and F5lat 
achieved the best decoding results in both animals, which was reflected in the steepest performance increase as a function of number of neurons included for decoding. The information content on these arrays was therefore significantly higher than on the supplemental arrays (AIPlat, F5med) (t-test, $\mathrm{p}<0.01)$. Please note that no statistical comparison was possible for the array AIPlat in animal $Z$ due to the small number of neurons detected. However, mean values were still smaller than in AIPmed, as shown in figure $5 \mathrm{c}$ (animal $Z$, cue). In animal $M$, the decoding performance of $F 5$ med was even lower than in the M1 arrays. This was surprising since the recording quality on this array was quite high.

Additional interesting observations were made for the M1 arrays. First, both arrays achieved performances above chance already during the planning epoch, indicating the presence of preparatory activity in M1. However, in both animals the more lateral array (M1lat) provided significantly better decoding accuracies during motor preparation than its medial counterpart (M1med). Very surprising though, we found that the F5lat array performed not only better than both M1 arrays during motor planning, but also during motor execution. In the hold epoch M1lat, M1med, and F5lat achieved best accuracies, however with different order in both animals.

When comparing the decoding performances across task epochs, we observed a strong role of AlPmed in motor preparation (cue and planning epoch), whereas decoding performance strongly decreased during motor execution (movement and holding epoch). In contrast, M1 showed a continuous increase in decoding performance over time, with best performance during the hold epoch, as expected.

Together, higher motor cortical areas in premotor and parietal cortex could be used to decode a wide range of grasping actions in 50 different object conditions. Decoding results from these areas were almost as high during motor preparation as during motor execution. Decoding from primary motor cortex, on the other hand, was strongest during grasp execution. Furthermore, we found strong differences between the sub-areas of F5 and AIP. Recording sides F5lat and AIPmed demonstrated most informative planning signals consistently in both animals. In particular F5lat was best suited for decoding during both motor preparation and execution. This area might therefore be a good target for a hybrid brain-computer interface that is capable of exploiting both: grasp planning and movement execution.

\section{Grip-type decoding}

One major goal of this study was to decode motor signals rather than visual object attributes. For this reason, we focused on the planning and hold epoch of the task that were performed in darkness. Furthermore and importantly, we also decoded the grip types applied to the objects based on the 
kinematic measures from the instrumented glove. This way, trials were assigned to specific grip types rather than individual objects.

For the classification of grip types, we recorded the 3D trajectories of 18 joint locations of the hand and arm with an electromagnetic tracking glove (figure 1a) (Schaffelhofer and Scherberger, 2012) in parallel to the neural data. This technology allowed us to record the movements continuously, even when fingers were hidden behind an object or obstacle. Furthermore, we used the recorded marker trajectories to drive a primate-specific musculoskeletal model (figure 6a) (Schaffelhofer et al., 2014). The model allowed extracting 27 DOF of the primate's upper extremity (figure 6b) that were subsequently used to classify the applied grips. For this analysis, we focused on the hold epoch, since it showed the highest variability of hand shapes under the most stable conditions. Figure $6 c$ presents the hand configurations of the hold epoch as principal component transforms with each the correctly performed trial represented as a single marker in PCA space (marker symbols as in figure $2 \mathrm{~b}$, marker size reflecting object size).

Using hierarchical cluster analysis, we then identified the 20 most different grip types from the multidimensional hand configuration dataset (27 DOF) of holding the 50 objects ten times (see Methods section). The resulting separable grip-type clusters are differentiated by color in figure $6 \mathrm{~d}$ and demonstrate highly variable hand configurations.

Furthermore, the hand configuration that each cluster represents is shown in figure 7a. Apparently, the high quality of the hand-tracking data allowed differentiating quite small grip differences. For example, grip type 1 and 2 were very similar in shape. However, they showed a minor but relevant difference: grip type 1 was applied to the small balls, which were the smallest objects of the set, whereas grip type 2 was applied to the small rings and required a slightly larger thumb-index aperture. Grips 3-6 represented whole-hand grips of different apertures, requiring one (i.e., grip 3) to 4 digits (i.e., grip 6). Grasping the large balls (i.e., grip 7) additionally required support from the little finger and a strong spread to enclose these large round objects. Grips 8-11 were applied to the long horizontal objects including bars and horizontal cylinders that needed variable apertures, not only of the digits $2-5$, but also of the thumb. For example, grip 8 was applied to a cylinder of smallest diameter, whereas grip 11 was applied to the thickest bar. Also, there was a high similarity between the classes 9 and 10. Both required similar apertures of thumb and index, however, the proximal and distal phalanges had to be more flexed for enclosing a cylinder (i.e., 10) than for the bars that required more extended fingers. A special hand configuration was applied to the average sized rings. In this case, the monkey was using a hook grip with the index finger to lift the object (i.e., grip 12). Grips 13-20 required variable amount of wrist rotation (see also $1^{\text {st }}$ PC in figure $6 \mathrm{~d}$ ). Minimal wrist rotation was applied when grasping the large cubes (i.e. 13), whereas the wrist was rotated to almost 
$180^{\circ}$ when the big cylinders were lifted from below (i.e. 20). Furthermore, grips 16 and 17 reflected precision and power grips applied to the handle. Index and thumb were used to perform the precision grip (grip 17), whereas all digits were used to enclose and pull the handle (grip 16). Finally, class 18 and 19 reflect the grips performed onto the vertical cylinders, which were similar to grips 8 and 10 for the horizontal cylinders, but with the wrist supinated by about $90^{\circ}$.
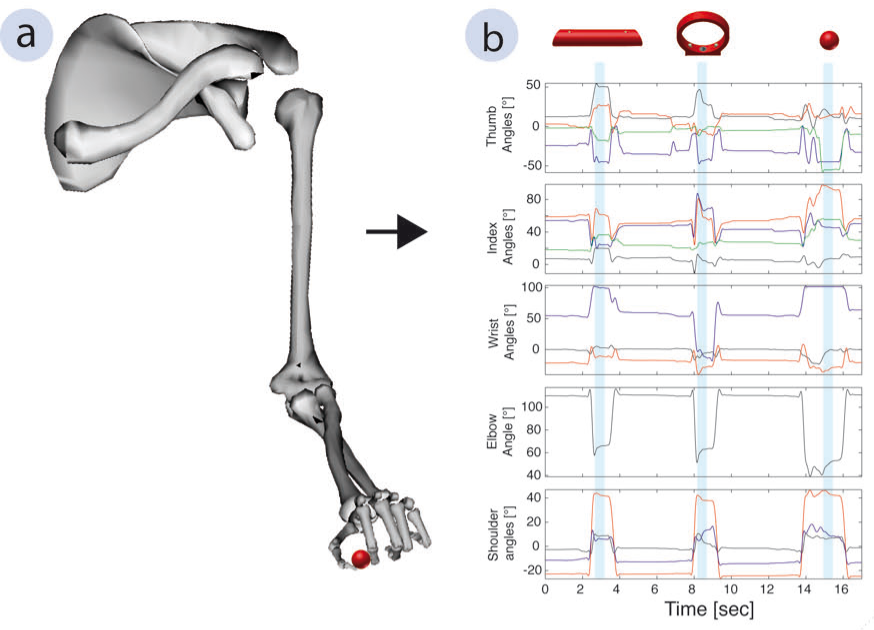

C

d

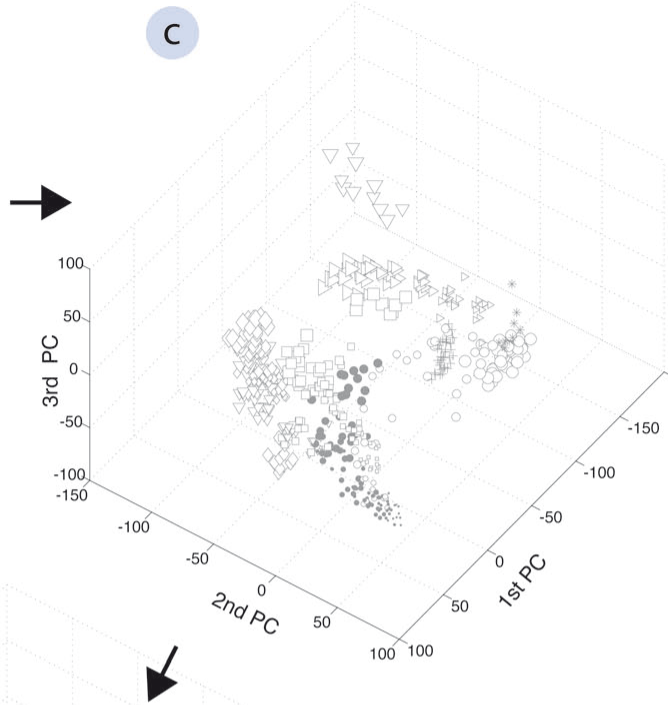

20

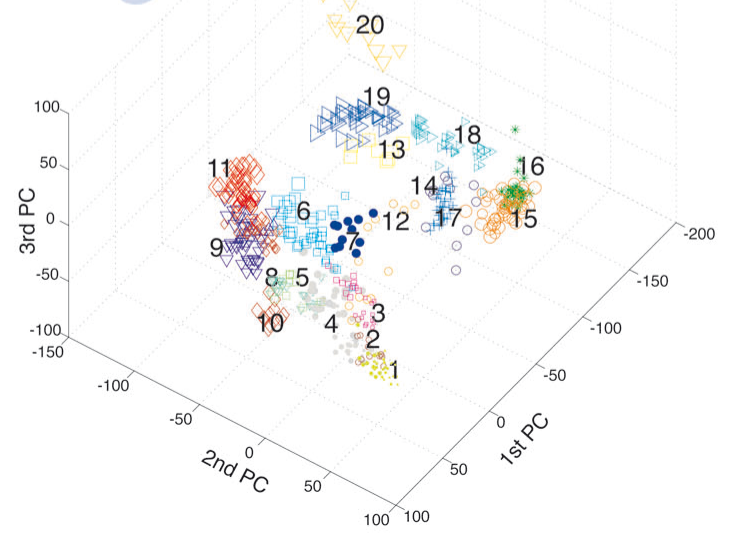

Figure 6. Grip type clustering. (a) Recorded joint positions of the upper limb were used to drive a $3 D$ musculoskeletal model. Applying the kinematics to the primate specific model allowed extracting joint angles of the hand and arm (27 DOF). A selection of features is shown in (b). Presented are from top to bottom: thumb and index angles (carpometacarpal adduction/abduction in black, carpometacarpal flexion/extension in red, proximal interphalangeal flexion/extension in blue, distal interphalangeal flexion/extension in green), wrist angles (deviation in black, flexion/extension in red, pronation/supination in blue), elbow angle (flexion in black), and shoulder angles (adduction/abduction in black, elevation in red, and rotation in blue). The hold epoch (used for classification) is highlighted in blue for grasping a horizontal bar, ring, and small ball. Subplot (c) illustrates the joint angles of the hold epoch as principal component (PC) transforms. Each symbol reflects an individual and correctly performed trial within the space of the first three PCs. Different symbols represent different object shapes, whereas their size reflects the object size. Applying hierarchical clustering to the multi-dimensional kinematic data allowed us to recluster the trials based on the applied grip type (d). The 20 most different hand configurations of the example session are numbered consecutively and trials from the same grip type class share the same color. 
Categorizing the trials based on the performed grips instead of the presented objects did not only improve the separation between visual and motor features, but also reduced redundancies within objects. For example, different objects that required the same or similar grips could be merged into the same cluster. Training the decoder on these hand-configuration classes readily allowed decoding these 20 grip types highly accurately. Figure 7a shows an example session in which hand configurations were decoded with an accuracy of $86 \%$ and $92 \%$ from the planning and hold epoch, respectively.

The independence of grip types from visual features was particularly apparent in two specific grip type classes: grip type 10 and 20 were applied to the same object, the big cylinder. The monkey decided to grasp this object in some instances from above with the hand pronated or in some trials from below with the hand in supination. Another example is the pair of grip 16 and 17 . Again, these grips were applied to the same object (the handle), but these grip types could be predicted with high accuracy already during the planning epoch (motor preparation). Both examples demonstrated that the decoder can differentiate these grips, even though they were performed on the same objects, thereby demonstrating object-independent decoding. However, a complete independent classification of objects and grips is not possible in general, since the shape of the hand is highly linked to the shape of the object being grasped (see figure $6 \mathrm{~d}$ ). Therefore, individual grip type classes often reflected particular objects.

Similar to object-decoding, the results of grip-type decoding across all recording session (figure $7 \mathrm{~b}$ ) demonstrated that decoding accuracy was highest when data from all cortical areas were considered, both during the planning ( $73 \pm 6.2 \%, 74.7 \pm 3.5 \%$, animal $\mathrm{Z}$ and $\mathrm{M}$ resp.) and hold epoch ( $82.15 \pm$ $5.0 \%, 89.2 \pm 1.7 \%$, animal $Z$ and $M$ resp.). Again, AlPmed and F5lat contributed most during the planning epoch, whereas M1lat and M1med predicted in both animals the grip types best during movement execution. However, across all electrode arrays F5lat achieved the highest performance when considering both planning or execution epoch.

These results demonstrate that higher cortical areas can indeed be used to decode complex hand configurations already during motor planning and with only slightly lower decoding performance than in the motor execution phase. This is impressive, since grip types were classified during the hold epoch and should therefore reflect the decoded hand configurations best. Nevertheless, the contribution of AIP and F5 during motor preparation led to a decoding performance that was on average only 8.6 and 14.5 percentage points smaller than during the hold epoch (animal $Z$ an $M$, respectively). 

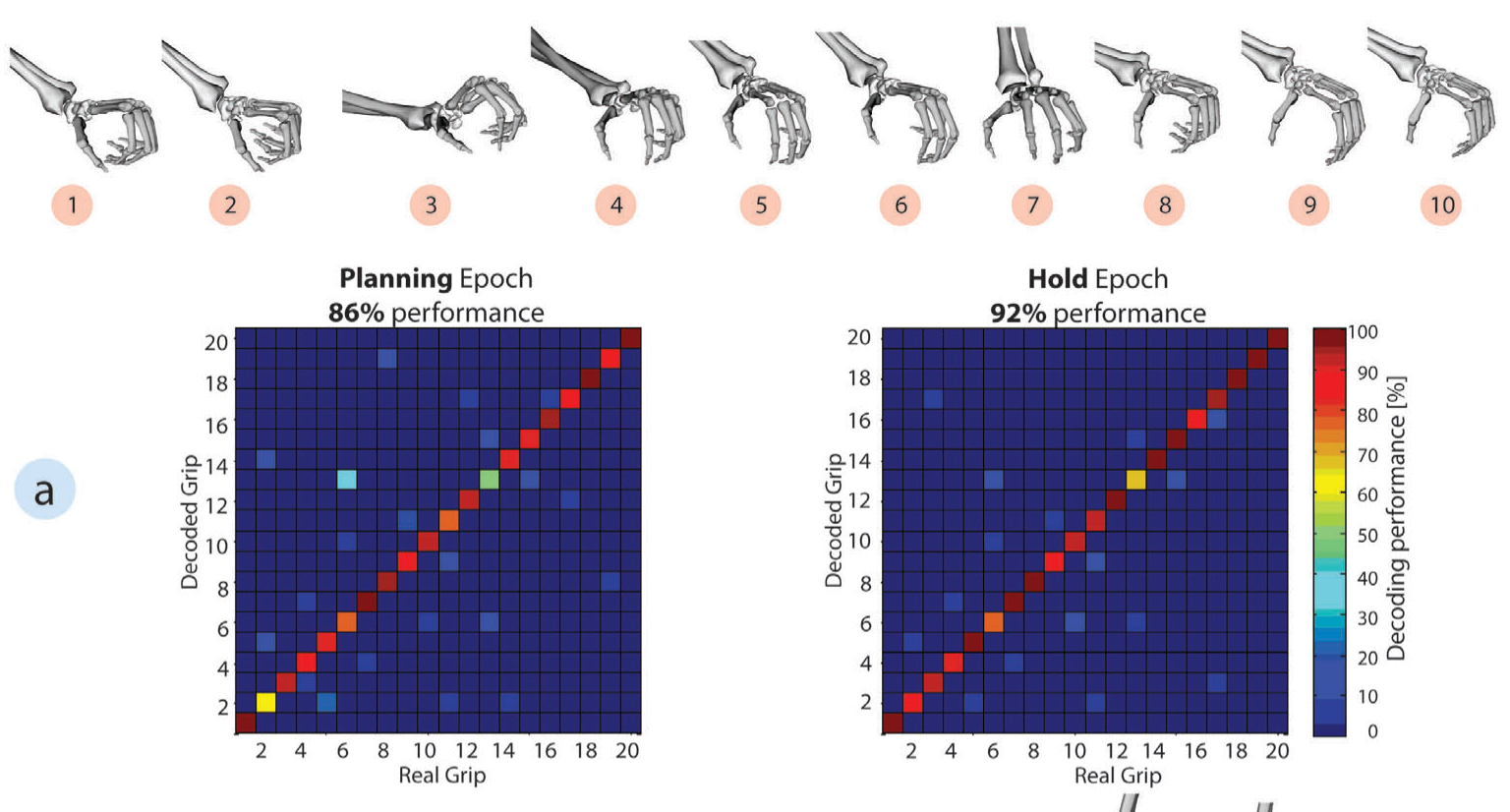

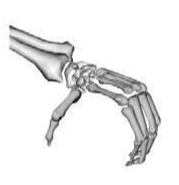

11

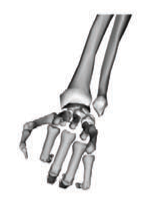

12

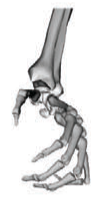

13

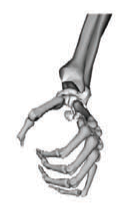

14

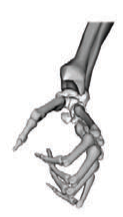

15

5

6

8

9

10
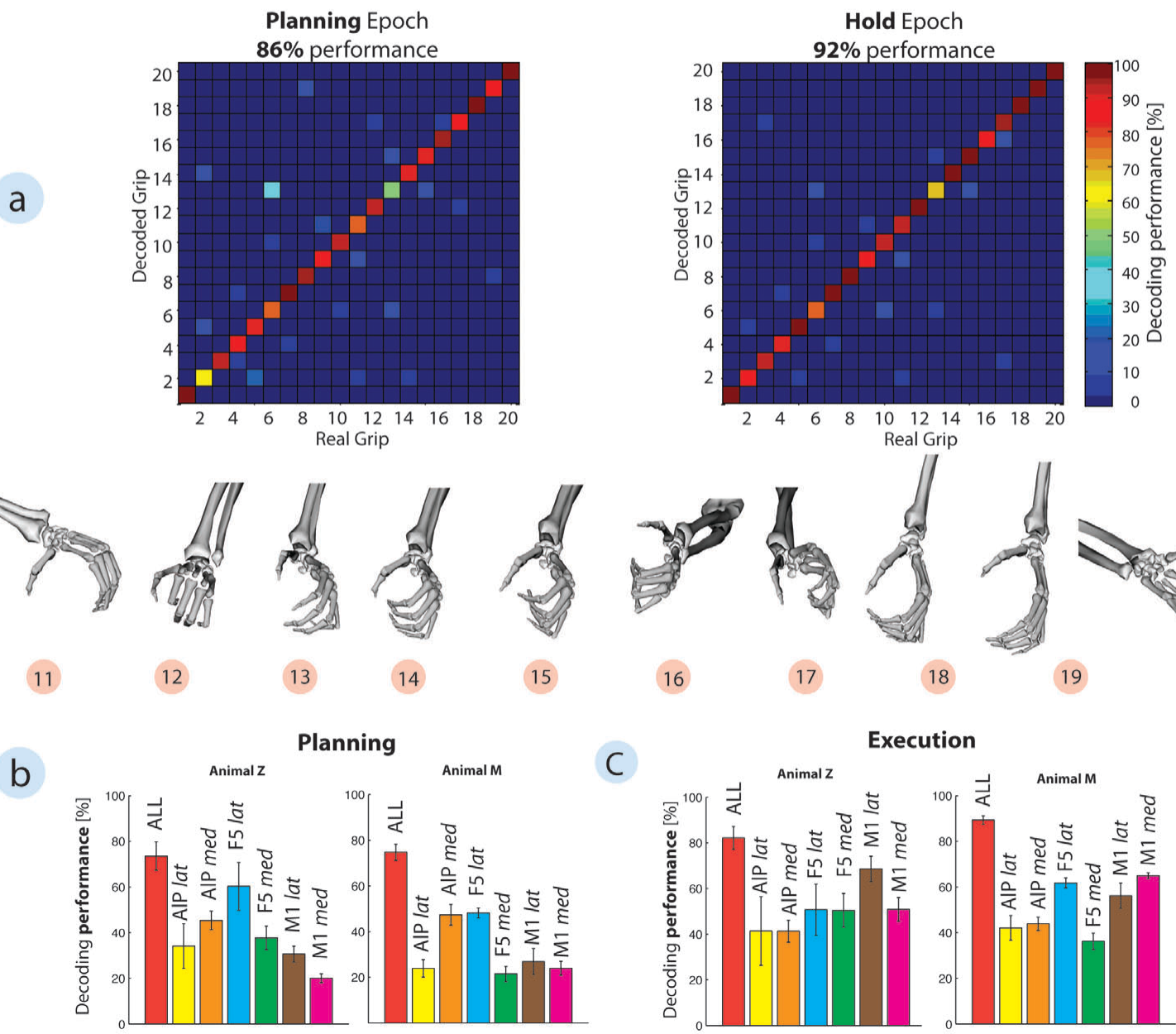

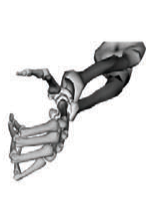

16

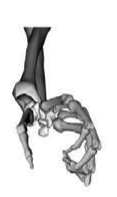

17

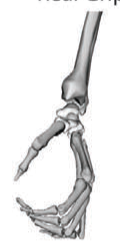

18

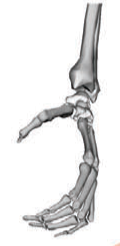

19

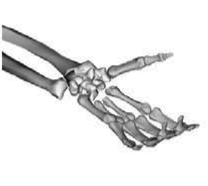

20

C

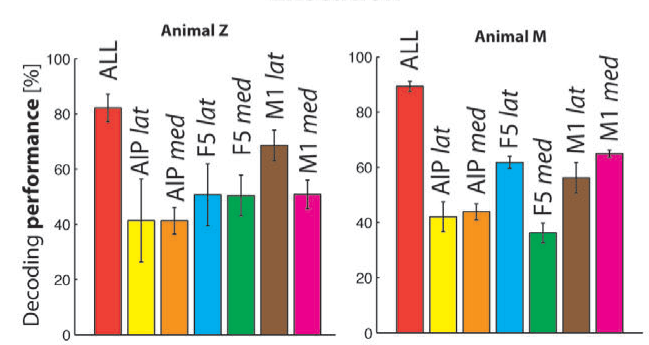

Figure 7. Grip-type decoding. (a) Hand configurations 1-20 and confusion matrices expressing decoding performance during the planning and hold epoch, respectively. Grip types were decoded using simultaneously recorded neurons from area AIP, F5, and M1. Hand configurations and grip type numbers as in figure 6. (b-c) Grip type decoding performances are summarized across all recording sessions for each implanted microelectrode array during the planning and hold epoch (mean \pm s.d.). Note: array specific decoding results were limited to a maximum of 30 neurons to allow a fair comparison of arrays. Color code as in figure $5 \mathrm{c}$.

\section{Spike sorting affects decoding performance}

For future real-time applications, the instantaneous processing of action potentials in large populations, i.e., spike sorting, might be difficult. The classification of action potentials to individual neurons can cause extensive computations, such as principal component transformation or template matching algorithms across a large number of channels. Previous studies have demonstrated minimal loss of decoding performance when advanced spike sorting methods were replaced by simple 
thresholding techniques (Gilja et al., 2011) or when spikes recorded from the same channel were merged to a single multi-unit (Gilja et al., 2012, Hochberg et al., 2012, Collinger et al., 2013). These procedures limit the number of available units to the number of electrodes and largely avoid the computational cost of spike sorting.

When comparing both methods in our decoding analysis, we found, as expected, better decoding accuracies when applying spike sorting instead of simple thresholds (figure 8): decoding accuracy increased on average by 9.9 and 8.8 percentage points during the planning and hold epoch, respectively, across all sessions and animals. Although these differences were significant (ANOVA, $p<0.01$ ), the clusters were still located close to unity line in the scatter plot, suggesting that simple thresholding could be used to decode a wide range of conditions with a negative effect of decoding accuracy of less than 10 percentage points.

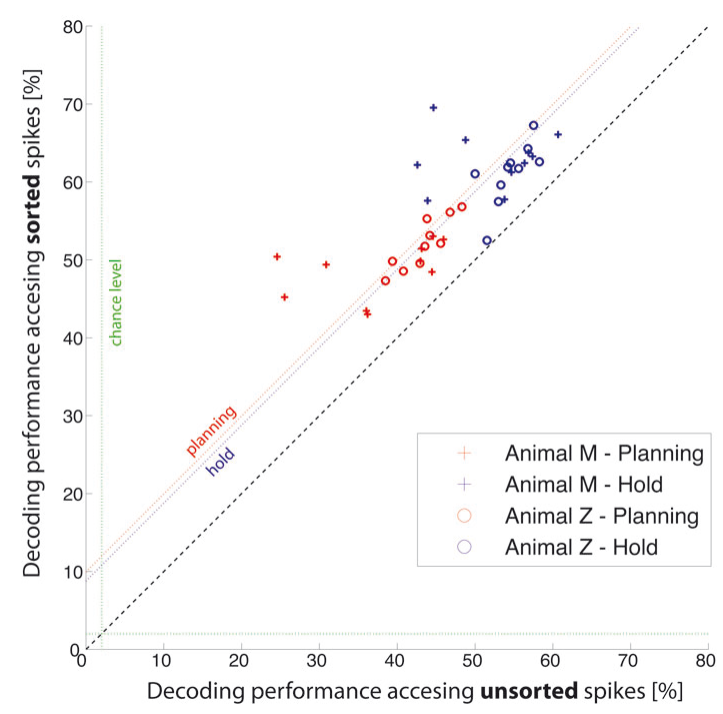

Figure 8. Spike sorting affects decoding accuracy. Scatter plot comparing the decoding performance using unsorted spiking activity ( $x$-axis) and sorted spiking activity ( $y$-axis). Symbols indicate results for both animals during the planning (red) and hold epoch (blue).

\section{Offline robotic control}

Finally, for future robotic applications, we also tested the translation of the decoded hand configurations into postures of an anthropomorphic arm and hand (figure 9). Because of noncongruent architectures and common under-actuation of currently available robotic hands (one motor actuates several DOF), such transformations are often non-trivial. Using a simple transformation method (see Methods), we could demonstrate the translation of the 20 grip type classes (defined by 27 DOF) in a 16 DOF robotic arm and hand (Barrett arm, 7 DOF; Schunk hand, 9 
DOF) (figure 9). Two problems were encountered in translating the grip. One was that the thumb abduction of the robot rotated about a different axis than the primate thumb (see figures 9a, inset, and figure 9b). Our approach was to visually match the ranges in which both thumbs coincided in orientation and restricting the robot movement to this range. The second problem concerned the execution of the ring- and little fingers, since both robotic fingers were actuated by only 1 DOF. This was solved by averaging the little and ring finger joint angles of the primate model (see figure 9c-e). Together, even though this robotic illustration was performed offline and rather qualitatively, it nevertheless demonstrates the feasibility of the primate hand model for future neuroprosthetic applications.
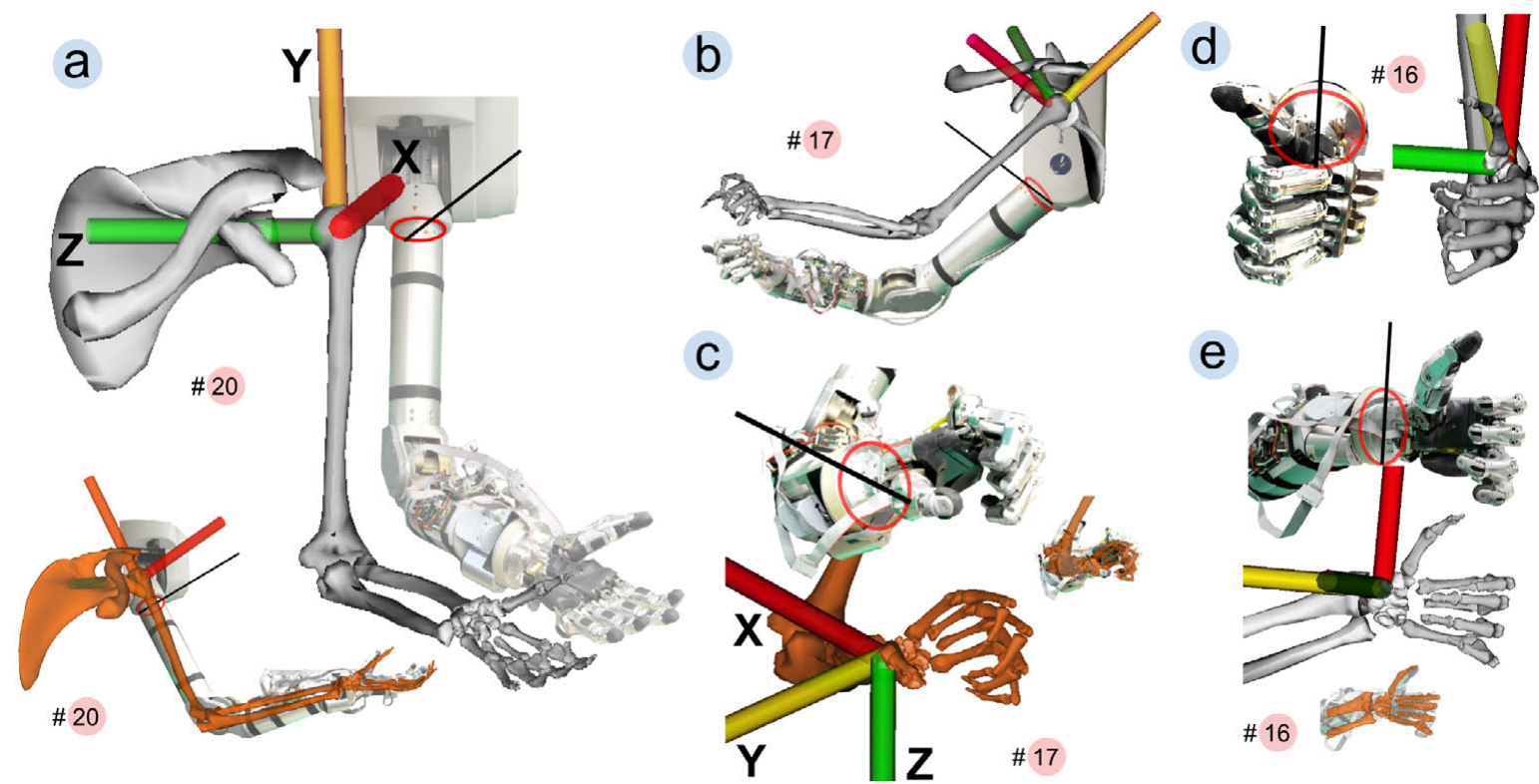

Figure 9: Execution of arm pose and grip type by a low-dimensional prosthetic device. Execution was compared to the primate skeletal model (SM). To infer the device's frame of reference from photographs, an oval (red) was drawn matching the circumference of its upper arm or wrist. The direction of the robot axis was estimated with a secant (black) cutting the oval in two equal parts and touching a physical marker painted on the robot. The model was manually aligned setting the SM's $X$ axis (red) parallel to the secant and the SM's $Y$ axis (yellow) parallel to the robot's upper or lower arm. (a) Reproduction of grip 20 (see figure 7) by the prosthetic device. The device can enact the hand and arm pose as rendered by the SM. Inset: medial view. (b-c) Arm and hand during grip 17. Digits 2-5 represent the grip well except at the distal phalanges that have no separate control on the robot. Inset: superposition of both grips. (d, e) Frontal and lateral view of grip 16 (as in figure 7). The grip is well represented except for the distal phalange angles. 


\section{Discussion}

The extensive experimental task design has let us record kinematics of the primate hand together with neural activity of the cortical areas AIP, F5, and M1 that are known to be involved in hand movement generation. From the planning and execution signals of these areas, we demonstrated accurate decoding of a wide range of hand configurations that animals used to grasp 50 different objects.

\section{Object based decoding}

As a first approach, we evaluated the decoding capabilities of AIP, F5, and M1 on the full set of 50 objects. This number of conditions was larger than in previous studies (Vargas-Irwin et al., 2010, Carpaneto et al., 2011, Carpaneto et al., 2012, Aggarwal et al., 2013) and caused a high variability of hand shapes, ranging from precision to power grips as well as many different grasp apertures (figure 6d). Grasp-planning areas AIP and F5 were capable of resolving the many grip type conditions even during movement preparation. Decoding performances during the planning phase were only moderately lower $(<15 \%)$ than in the movement epoch. Selective responses at the population level (Fig. 4) and distinct modulations of individual neurons (Fig. 3) are able to explain the planning quality of the AIP and F5 population.

Although the decoding performance was on average about 30 times larger than chance (execution epoch), the actual correlation between real and decoded conditions was even higher. Most of the decoding errors were made to adjacent objects of similar shape and size (figure 5). This closeness of grip-conditions was intended: in contrast to previous studies that classified a few largely different grip types (Townsend et al., 2011, Carpaneto et al., 2012), we have introduced object similarities to evaluate the nature of the neural signals at the various recording sites.

Training the Bayesian classifier separately with neuronal ensembles from each individual electrodearray revealed significant differences across the recorded populations. In both animals the arrays F5lat and AIPmed carried significantly more information about the upcoming grip than their complementary array in the same area (F5med and AIPlat). Interestingly, we also found differences in terms of planning activity between the two M1 arrays. M1lat, located at the level of the principle sulcus, achieved higher decoding performances during grasp planning than M1med. However, these effects were marginal in comparison to the activity during movement execution, when the majority of neurons at both sites (M1lat and M1med) showed strong selective responses (see figure 4), in line with the known direct cortico-motoneuronal connections of M1 to the distal limb musculature (Rathelot and Strick, 2009). These results clearly highlight the importance of M1 for hand movement control and its suitability for potential neuroprosthetic applications with larger number of objects. 


\section{Grip-type based decoding}

Area AIP and F5 are part of the frontoparietal network that is highly relevant for transforming visual attributes of objects into motor commands for grasping (Jeannerod et al., 1995, Luppino et al., 1999, Rizzolatti and Luppino, 2001, Brochier and Umilta, 2007). In both areas, preparatory neuronal activities have been reported that reflected context specific object information as well as 2- and 3dimensional object features (Murata et al., 2000, Raos et al., 2006, Baumann et al., 2009, Fluet et al., 2010, Theys et al., 2012a, Theys et al., 2012b, Romero et al., 2014). In agreement with these findings, neurons from AIP and F5 responded selectively to the presentation of various objects (figure 4).

To demonstrate the capability to decode motor plans rather than visual attributes, we evaluated the preparation activity during the planning epoch when animals were in complete darkness. Furthermore, we classified neural activity based on the applied grip type rather than the observed object. For this, we tracked finger, hand, and arm movements with an instrumented glove equipped with electromagnetic sensors (Schaffelhofer and Scherberger, 2012). From the 3D marker trajectories we then extracted the joint angles in 27 DOF and classified them into 20 grip type classes. This classification method did not only create classes based on the applied grip, but also reduced redundancies among conditions, since some objects were grasped with the same grip. We have selected a relatively high number of grip types to test the limits of the decoder and signals. However, a lower number of hand configurations would be sufficient in daily life and could potentially increase the decoding performance (Bullock et al., 2013).

The rather good performance for these grip type conditions clearly demonstrated, to our knowledge for the first time, that a large number of hand configurations can be precisely decoded from both motor planning and motor execution signals: whereas AIPmed and F5lat contributed strongest during movement preparation, M1 showed the best performance during object grasping.

Because animals were allowed to grasp the objects intuitively, some objects like the horizontal cylinders were sometimes grasped with alternative grips (e.g., with pronated vs. supinated hand). Although the object attributes were identical in such cases, we were able to classify the correct grip, therefore demonstrating the decoding of a motor plan rather than objects.

However, a strict separation between visual and motor attributes is generally not possible. This conclusion is supported by the PCA analysis that demonstrated a clear link between object shape and the applied grip type (figure 6d). This statement is relevant for decoding applications as well as for our general understanding of grasp coding in AIP and F5. Clearly, the simple neuronal responses and decoding analysis presented here cannot reveal the real nature of the neural signals in terms of their object or motor representations. Since either visual object features or the intended motor plan could 
generate the observed object selectivity, further investigations of the neural state space are necessary to address these questions.

\section{Implications on neuroprosthetics}

Previous work has presented striking examples of neural interfaces for the control of arm prosthetics. However, most of these studies, did not consider dexterous control of an anthropomorphic hand. Instead, they implemented 1-dimensional controls for simple grippers that essentially could be opened and closed (Velliste et al., 2008). Whereas hand orientation was not, or only manually controlled in in the past (Hochberg et al., 2012), a recent study achieved an additional neural control of the wrist (Collinger et al., 2013). Although some offline studies demonstrated a continuous reconstruction of finger and hand movements (Vargas-Irwin et al., 2010, Bansal et al., 2012, Aggarwal et al., 2013), none of them demonstrated the capability for closed-loop applications, since they were decoded in parallel to the actual movement. Therefore, the neural control of the many DOF of the hand under visual guidance remains the major challenge. Accessing higher cortical areas that reflect motor intentions rather than individual joint angle control might help reducing the dimensionality problem for real-time applications (Carpaneto et al., 2011, Townsend et al., 2011). Here, we demonstrated the decoding of a wide range of complex hand configurations from motor preparatory activity, ranging from precision grips to power grips.

Furthermore, as a test for prospective real-time applications, we illustrated the possibility of translating hand postures to an anthropomorphic 16 DOF hand and arm. Inverse kinematics and a linear translation of hand configurations allowed executing a total of 20 grip types on the robotic device. This offline test demonstrated the possibility of physically executing complex hand configurations as decoded from neuronal planning and execution signals.

Although decoding motor intensions significantly reduced the decoding complexity of the primate hand, it is important to note that such an open-loop approach could not work stand-alone. For realtime applications, the instant processing of neural activity for aperture control and error correction would be required. One possibility would be a hybrid neural interface that accesses both planning and motor execution signals for grasping. Such an approach could consist of three major steps: (1) detecting the planning state before movement onset (Aggarwal et al., 2013), (2) decoding the intended grip type from preparatory activity, and (3) closing the aperture of the decoded hand configuration with continuous decoders (e.g. Kalman-filter) in closed- loop applications under visual guidance (Collinger et al., 2013). In our study the ventral premotor cortex showed similar or even better performance during movement execution than primary motor cortex. The redundancy between both interconnected areas (Dum and Strick, 2005) was already reported in previous 
decoding studies (Aggarwal et al., 2013). However, driving a hybrid neural interface with access to planning and motor activity could benefit from both areas and lead to a significant increase in decoding performance and usability. Therefore, motor execution signals may not necessarily have to originate from motor cortex. As shown in figure $5 c$, the lateral part of $F 5$ demonstrated the best performance across planning and motor epochs and hence might be well suited for this kind of applications. 


\section{References}

Aggarwal V, Mollazadeh M, Davidson AG, Schieber MH, Thakor NV (2013) State-based decoding of hand and finger kinematics using neuronal ensemble and LFP activity during dexterous reach-to-grasp movements. Journal of neurophysiology 109:3067-3081.

Andersen RA, Hwang EJ, Mulliken GH (2010) Cognitive neural prosthetics. Annu Rev Psychol 61:169-190, C161-163.

Anderson KD (2004) Targeting recovery: priorities of the spinal cord-injured population. J Neurotrauma 21:1371-1383.

Bansal AK, Truccolo W, Vargas-Irwin CE, Donoghue JP (2012) Decoding 3D reach and grasp from hybrid signals in motor and premotor cortices: spikes, multiunit activity, and local field potentials. Journal of neurophysiology 107:1337-1355.

Baumann MA, Fluet MC, Scherberger H (2009) Context-specific grasp movement representation in the macaque anterior intraparietal area. The Journal of neuroscience 29:6436-6448.

Belmalih A, Borra E, Contini M, Gerbella M, Rozzi S, Luppino G (2009) Multimodal architectonic subdivision of the rostral part (area F5) of the macaque ventral premotor cortex. J Comp Neurol 512:183-217.

Borra E, Belmalih A, Calzavara R, Gerbella M, Murata A, Rozzi S, Luppino G (2008) Cortical connections of the macaque anterior intraparietal (AIP) area. Cereb Cortex 18:10941111.

Borra E, Belmalih A, Gerbella M, Rozzi S, Luppino G (2010) Projections of the hand field of the macaque ventral premotor area F5 to the brainstem and spinal cord. J Comp Neurol 518:2570-2591.

Brochier T, Umilta MA (2007) Cortical control of grasp in non-human primates. Curr Opin Neurobiol 17:637-643.

Bullock IM, Zheng JZ, De La Rosa S, Guertler C, Dollar AM (2013) Grasp frequency and usage in daily household and machine shop tasks. IEEE transactions on haptics 6:296-308.

Carpaneto J, Raos V, Umilta MA, Fogassi L, Murata A, Gallese V, Micera S (2012) Continuous decoding of grasping tasks for a prospective implantable cortical neuroprosthesis. Journal of neuroengineering and rehabilitation 9:84.

Carpaneto J, Umilta M, Fogassi L, Murata A, Gallese V, Micera S, Raos V (2011) Decoding the activity of grasping neurons recorded from the ventral premotor area F5 of the macaque monkey. Neuroscience 188:80-94.

Collinger JL, Wodlinger B, Downey JE, Wang W, Tyler-Kabara EC, Weber DJ, McMorland AJ, Velliste M, Boninger ML, Schwartz AB (2013) High-performance neuroprosthetic control by an individual with tetraplegia. Lancet 381:557-564.

National Research Council (2003) Guidelines for the care and use of mammals in neuroscience and behavioral research. Washington, D.C.: National Academies Press.

Delp SL, Anderson FC, Arnold AS, Loan P, Habib A, John CT, Guendelman E, Thelen DG (2007) OpenSim: open-source software to create and analyze dynamic simulations of movement. IEEE transactions on bio-medical engineering 54:1940-1950.

Dum RP, Strick PL (2005) Frontal lobe inputs to the digit representations of the motor areas on the lateral surface of the hemisphere. J Neurosci 25:1375-1386.

Fluet MC, Baumann MA, Scherberger H (2010) Context-specific grasp movement representation in macaque ventral premotor cortex. The Journal of neuroscience 30:15175-15184.

Ganguly K, Carmena JM (2009) Emergence of a stable cortical map for neuroprosthetic control. PLoS Biol 7:e1000153.

Gilja V, Chestek CA, Diester I, Henderson JM, Deisseroth K, Shenoy KV (2011) Challenges and opportunities for next-generation intracortically based neural prostheses. Biomedical Engineering, IEEE Transactions on 58:1891-1899.

Gilja V, Nuyujukian P, Chestek CA, Cunningham JP, Yu BM, Fan JM, Churchland MM, Kaufman MT, Kao JC, Ryu SI, Shenoy KV (2012) A high-performance neural prosthesis enabled by control algorithm design. Nat Neurosci 15:1752-1757. 
Hatsopoulos NG, Donoghue JP (2009) The science of neural interface systems. Annual review of neuroscience 32:249-266.

Hochberg LR, Bacher D, Jarosiewicz B, Masse NY, Simeral JD, Vogel J, Haddadin S, Liu J, Cash SS, van der Smagt P, Donoghue JP (2012) Reach and grasp by people with tetraplegia using a neurally controlled robotic arm. Nature 485:372-375.

Jeannerod M, Arbib MA, Rizzolatti G, Sakata H (1995) Grasping objects: the cortical mechanisms of visuomotor transformation. Trends Neurosci 18:314-320.

Kim SP, Simeral JD, Hochberg LR, Donoghue JP, Friehs GM, Black MJ (2011) Point-and-click cursor control with an intracortical neural interface system by humans with tetraplegia. IEEE transactions on neural systems and rehabilitation engineering : a publication of the IEEE Engineering in Medicine and Biology Society 19:193-203.

Kuiken TA, Li G, Lock BA, Lipschutz RD, Miller LA, Stubblefield KA, Englehart KB (2009) Targeted muscle reinnervation for real-time myoelectric control of multifunction artificial arms. JAMA : the journal of the American Medical Association 301:619-628.

Lehmann SJ, Scherberger H (2013) Reach and gaze representations in macaque parietal and premotor grasp areas. The Journal of neuroscience 33:7038-7049.

Luppino G, Murata A, Govoni P, Matelli M (1999) Largely segregated parietofrontal connections linking rostral intraparietal cortex (areas AIP and VIP) and the ventral premotor cortex (areas F5 and F4). Experimental brain research Experimentelle Hirnforschung Experimentation cerebrale 128:181-187.

Murata A, Fadiga L, Fogassi L, Gallese V, Raos V, Rizzolatti G (1997) Object representation in the ventral premotor cortex (area F5) of the monkey. J Neurophysiol 78:2226-2230.

Murata A, Gallese V, Luppino G, Kaseda M, Sakata H (2000) Selectivity for the shape, size, and orientation of objects for grasping in neurons of monkey parietal area AIP. J Neurophysiol 83:2580-2601.

Musallam S, Corneil BD, Greger B, Scherberger H, Andersen RA (2004) Cognitive control signals for neural prosthetics. Science 305:258-262.

Paul RP (1982) Robot Manipulators: Mathematics, Programming, and Control: MIT Press.

Quiroga RQ, Nadasdy Z, Ben-Shaul Y (2004) Unsupervised spike detection and sorting with wavelets and superparamagnetic clustering. Neural Comput 16:1661-1687.

Raab FH, Blood EB, Steiner TO, Jones HR (1979) Magnetic Position and Orientation Tracking System. Ieee T Aero Elec Sys 15:709-718.

Raos V, Umilta MA, Murata A, Fogassi L, Gallese V (2006) Functional properties of graspingrelated neurons in the ventral premotor area F5 of the macaque monkey. Journal of neurophysiology 95:709-729.

Rathelot JA, Strick PL (2009) Subdivisions of primary motor cortex based on corticomotoneuronal cells. Proc Natl Acad Sci U S A 106:918-923.

Rizzolatti G, Camarda R, Fogassi L, Gentilucci M, Luppino G, Matelli M (1988) Functional organization of inferior area 6 in the macaque monkey. II. Area F5 and the control of distal movements. Exp Brain Res 71:491-507.

Rizzolatti G, Luppino G (2001) The cortical motor system. Neuron 31:889-901.

Romero MC, Pani P, Janssen P (2014) Coding of shape features in the macaque anterior intraparietal area. J Neurosci 34:4006-4021.

Schaffelhofer S, Sartori M, Scherberger H, Farina D (2014) Musculoskeletal Representation of a Large Repertoire of Hand Grasping Actions in Primates. IEEE Trans Neural Syst Rehabil Eng. Advance online publication. Retrieved November 25, 2014. doi:101109/TNSRE20142364776.

Schaffelhofer S, Scherberger H (2012) A new method of accurate hand- and arm-tracking for small primates. Journal of neural engineering 9:026025.

Scherberger H (2009) Neural Prostheses for Reaching. In: Encyclopedia of Neuroscience, vol. 6 (Squire, L. R., ed), pp 213-220 Oxford: Academic Press.

Scherberger H, Jarvis MR, Andersen RA (2005) Cortical local field potential encodes movement intentions in the posterior parietal cortex. Neuron 46:347-354.

Schieber MH (1991) Individuated finger movements of rhesus monkeys: a means of quantifying the independence of the digits. Journal of neurophysiology 65:1381-1391. 
Schieber MH, Hibbard LS (1993) How somatotopic is the motor cortex hand area? Science 261:489-492.

Snoek GJ, MJ IJ, Hermens HJ, Maxwell D, Biering-Sorensen F (2004) Survey of the needs of patients with spinal cord injury: impact and priority for improvement in hand function in tetraplegics. Spinal cord 42:526-532.

Subasi E, Townsend B, Scherberger H (2010) In search of more robust decoding algorithms for neural prostheses, a data driven approach. In: Engineering in Medicine and Biology Society (EMBC), 2010 Annual International Conference of the IEEE, pp 4172-4175: IEEE.

Taylor DM, Tillery SI, Schwartz AB (2002) Direct cortical control of 3D neuroprosthetic devices. Science 296:1829-1832.

Theys T, Pani P, van Loon J, Goffin J, Janssen P (2012a) Selectivity for three-dimensional shape and grasping-related activity in the macaque ventral premotor cortex. J Neurosci 32:12038-12050.

Theys T, Srivastava S, van Loon J, Goffin J, Janssen P (2012b) Selectivity for three-dimensional contours and surfaces in the anterior intraparietal area. Journal of Neurophysiology 107:995-1008.

Townsend BR, Subasi E, Scherberger H (2011) Grasp movement decoding from premotor and parietal cortex. The Journal of neuroscience : the official journal of the Society for Neuroscience 31:14386-14398.

Vargas-Irwin CE, Shakhnarovich G, Yadollahpour P, Mislow JM, Black MJ, Donoghue JP (2010) Decoding complete reach and grasp actions from local primary motor cortex populations. The Journal of neuroscience 30:9659-9669.

Velliste M, Perel S, Spalding MC, Whitford AS, Schwartz AB (2008) Cortical control of a prosthetic arm for self-feeding. Nature 453:1098-1101. 



\section{Discussion}

The defined goal of this dissertation was to investigate and compare the neuronal representation of hand movements in cortical areas AIP, F5, and M1. For this, a new experimental setup was built that allowed simultaneous recording of spiking activity from 192 channels together with kinematics of the primate hand and arm, and a task was designed in which macaque monkeys grasped a total of 50 different objects. The high variability of visual stimuli (object attributes) and motor responses (applied grip types) allowed us to describe the distinct roles of AIP, F5, and M1 in visuo-motor processing. Furthermore, the grip types applied on the full set of objects could be successfully decoded from the neural activity of these areas. In this final section, the technologies and findings of the four studies reported in this thesis are summarized and briefly discussed in a general context.

In the first part of this dissertation, a novel key technology developed for the investigation of hand movements was presented: a hand-tracking device for small primates (Schaffelhofer and Scherberger, 2012). This technology was required for comparing and correlating neural signals of the brain with the kinematics of the arm and hand.

Although many of such hand-tracking devices are available for humans (Harvill et al., 1992, Dipietro et al., 2003, Simone et al., 2007, Gentner and Classen, 2009), applications in non-human primates are rare (Overduin et al., 2010, VargasIrwin et al., 2010). This necessitated the development of a hand-tracking prototype (soft- and hardware) that fulfilled the requirements for experiments with monkeys: (1) compact size, (2) robustness (3) high spatial and (4) temporal resolution, and most importantly, (5) reliability. All of these claims could be implemented by building an instrumented glove equipped with seven electro-magnetic sensors (WAVE, Northern Digital Inc, Waterloo, CA). In contrast to other technologies that can either provide 
the orientation or the spatial position in space (Overduin et al., 2010, Vargas-Irwin et al., 2010), sensor coils give access to both. The high information content provided by the sensors in combination with a novel computational model, which exploited the constraints of finger and hand anatomy, allowed describing the motion of the upper limb in 27 DOF. To our knowledge, this ratio between DOF and number of sensors is higher than for any other published or commercially available method.

Although the reduced number of sensors required more computational effort, the usability was significantly increased. Less sensors and weight made the glove more tolerable for the animals. Consequently, the glove could be used on a daily basis and was reliable within recording sessions lasting up to 2 hours.

Furthermore, the applied magnetic-based sensor technology did not depend on line-of-sight to a camera as optical systems do (Vargas-Irwin et al., 2010, Aggarwal et al., 2013). This feature was extremely important for hand tracking in grasping experiments, since the manipulation of objects can easily occlude sensors or markers placed on the fingers or hand. Using electro-magnetic coils allowed to continuously track sensor trajectories, even if they were located behind the hand or one of the objects.

Important for future studies, the control software for the instrumented glove was implemented as a graphical user interface. The menu-guided program enables users to intuitively set up and monitor experiments. Furthermore, a wide range of interfaces allow transferring the measured hand kinematics to several electrophysiological recording systems for a synchronous data acquisition together with neural data (e.g., Cerebus, Blackrock; TDT). The software package will be shared with the research community.

Together, the novel computational model and data glove turned out to be a reliable technique for finger, hand, and arm tracking of primates using a minimal number of sensors. The measured kinematics provided the basis for all subsequent studies presented in this thesis. 
The second part of this dissertation presented a 3D musculoskeletal model of the primate upper (Schaffelhofer et al., 2014), implemented in OpenSim (Delp et al., 2007). The goal of this study was to translate the marker trajectories recorded with the instrumented glove into anatomically correct joint angles and muscle tendon length.

Previous work has studied the dynamics of the musculoskeletal system with a main focus on limb and joint kinematic variables such as hand velocity, direction, and joint angular position (Vargas-Irwin et al., 2010, Zhuang et al., 2010, Aggarwal et al., 2013). However, these methodologies have never taken into account the kinematic behavior of the underlying musculotendon units spanning the hand and the upper extremity joints during reach-to-grasp movements. To address this question, a generic musculoskeletal model of the human upper extremity (Holzbaur et al., 2005) was scaled and morphed to accurately match the primate specific anatomy of each individual monkey. Simulations based on the model and the real marker trajectories made it possible to extract non-invasively the joint angles in 27 degrees of freedom (DOF) and the instantaneous length of 50 muculotendon units.

Having simultaneous access to the joint angle and muscletendon description of the hand allowed a direct comparison between both domains. Most interestingly, we could demonstrate a more compact representation of the grasp movements when they were expressed in the muscle kinematics domain than in the joint angle domain. This lower dimensional representation could reflect the synergistic design of the muscular system of the hand and arm with many muscles spanning multiple joints. In this respect, our musculoskeletal model could serve as a template for the development of novel neurorehabiliation technologies: our findings suggest that prosthetic hands that are controlled by artificial tendons, rather than by individual joints, require lower dimensional control (Controzzi et al., 2010). 
Furthermore, the 3D musculoskeletal model provided an essential tool for the following neuronal studies of this thesis. Marker trajectories could be translated into anatomically accurate joint angles via inverse kinematics and vice versa. This allowed visualizing and expressing movements in the multi-joint domain of the hand and arm and facilitated a direct comparison with the neuronal state spaces of specific brain areas.

The third study of this thesis investigated the neuronal representation of hand actions in the macaque brain areas AIP, F5, and M1. Previous studies reported selective neuronal responses in these areas when objects were visually presented and grasped (Murata et al., 1997, Murata et al., 2000, Raos et al., 2006, Umilta et al., 2007, Baumann et al., 2009, Fluet et al., 2010). Although these findings provided strong evidence for the processing of visuomotor transformations, it remained unclear how visual and motor information is encoded at the neuronal population level.

To address this question, two macaque monkeys were trained to grasp 50 different objects in a delayed grasping task while we simultaneously recorded their neuronal activity from 192 channels together with the kinematics acquired from the instrumented glove. The extensive variability of visual stimuli and motor responses allowed separating and describing the multidimensional visual and motor features at the neuronal population level.

In agreement with anatomical studies (Nakamura et al., 2001, Borra et al., 2008), AIP was identified as a visual area that was representing objects in visual terms. Its neural population differentiated objects primarily based on their shape and secondarily on their size. Although these visual attributes could be observed during both object presentation and motor epochs, we could identify shifts in the neural population state when the same object was grasped with alternative grips. Based on how these modulations appeared at the neuronal population of AIP, we hypothesize 
that they reflect different visual attributes of the same object that are relevant for grasping. These findings are in agreement with the current understanding of AIP that has been associated with the visual coding of objects and the extraction of object affordances (Fagg and Arbib, 1998, Rizzolatti and Luppino, 2001, Baumann et al., 2009).

In contrast to AIP, area F5 primarily coded the objects in motor terms, although individual neurons could code visual information. Features like wrist orientation or grip aperture could be observed in part already during motor preparation and became apparent most distinctively during motor execution. However, highest resemblance of neural population activity with the measured kinematics of hand and arm configurations was observed in the cortical hand area of M1. We demonstrated that the neuronal space of M1 precisely reflects the multi-joint representation of the hand. These findings are in perfect agreement with anatomical studies demonstrating direct connections of the bank of M1 to alpha motor neurons in the spinal cord controling distal hand muscles (Rathelot and Strick, 2009). Together, the presented results revealed distinct roles of AIP, F5, and M1 at their population level and significantly improved our understanding of how visuomotor transformations are processed in the brain to generate hand actions.

The population analysis performed in AIP, F5 and M1 demonstrated the coding of grasp relevant information. In study 4, we accessed the neuronal planning and execution signals from these areas in order to decode the many hand configurations applied on the full set of 50 objects (Schaffelhofer et al., in press). In contrast to previous studies that predicted largely different grip types (Subasi et al., 2010, Carpaneto et al., 2011, Townsend et al., 2011), we demonstrated the possibility to decode complex hand shapes during both motor execution and motor preparation epochs. As a final test for future real time applications, we demonstrated that the decoded grip types could be executed by an anthropomorphic robotic hand. 
These results could be relevant for the development of neuroprosthetic devices: first, we could identify highest decoding capabilities during movement planning and execution epochs in the lateral recording site of F5 (F5a). Hybrid brain computer interfaces that access planning and execution signals might strongly benefit from spiking activity recorded in this area. Second, decoding of grip types, rather than individual joint angles of the hand, could help reducing the decoding complexity for neuroprosthetic application that have so far not accomplished full control of the complex versatility of the primate hand (Hochberg et al., 2012, Collinger et al., 2013). 


\section{Bibliography}

Aggarwal V, Mollazadeh M, Davidson AG, Schieber MH, Thakor NV (2013) State-based decoding of hand and finger kinematics using neuronal ensemble and LFP activity during dexterous reach-to-grasp movements. Journal of Neurophysiology 109:3067-3081.

Andersen RA, Hwang EJ, Mulliken GH (2010) Cognitive neural prosthetics. Annual review of psychology 61:169-190, C161-163.

Batista AP, Buneo CA, Snyder LH, Andersen RA (1999) Reach plans in eye-centered coordinates. Science 285:257-260.

Batista AP, Santhanam G, Yu BM, Ryu SI, Afshar A, Shenoy KV (2007) Reference frames for reach planning in macaque dorsal premotor cortex. Journal of Neurophysiology 98:966-983.

Baumann MA, Fluet MC, Scherberger H (2009) Context-specific grasp movement representation in the macaque anterior intraparietal area. The Journal of Neuroscience 29:6436-6448.

Biederman I (1987) Recognition-by-components: a theory of human image understanding. Psychological review 94:115-147.

Blasdel GG (1992) Orientation selectivity, preference, and continuity in monkey striate cortex. The Journal of Neuroscience 12:3139-3161.

Borra E, Belmalih A, Calzavara R, Gerbella M, Murata A, Rozzi S, Luppino G (2008) Cortical connections of the macaque anterior intraparietal (AIP) area. Cerebral cortex 18:1094-1111.

Borra E, Belmalih A, Gerbella M, Rozzi S, Luppino G (2010) Projections of the hand field of the macaque ventral premotor area F5 to the brainstem and spinal cord. Journal of comparative psychology 518:2570-2591.

Brochier T, Umilta MA (2007) Cortical control of grasp in non-human primates. Current opinion in neurobiology 17:637-643.

Buneo CA, Jarvis MR, Batista AP, Andersen RA (2002) Direct visuomotor transformations for reaching. Nature 416:632-636.

Carpaneto J, Raos V, Umilta MA, Fogassi L, Murata A, Gallese V, Micera S (2012) Continuous decoding of grasping tasks for a prospective implantable cortical neuroprosthesis. Journal of neuroengineering and rehabilitation 9:84.

Carpaneto J, Umilta M, Fogassi L, Murata A, Gallese V, Micera S, Raos V (2011) Decoding the activity of grasping neurons recorded from the ventral premotor area F5 of the macaque monkey. Neuroscience 188:80-94.

Castiello U (2005) The neuroscience of grasping. Nature Reviews Neuroscience 6:726736.

Cheney PD, Fetz EE (1980) Functional Classes of Primate Corticomotoneuronal Cells and Their Relation to Active Force. Journal of Neurophysiology 44:773-791.

Collinger JL, Wodlinger B, Downey JE, Wang W, Tyler-Kabara EC, Weber DJ, McMorland AJ, Velliste M, Boninger ML, Schwartz AB (2013) High-performance neuroprosthetic control by an individual with tetraplegia. Lancet 381:557-564.

Controzzi M, Cipriani C, Jehenne B, Donati M, Carrozza MC (2010) Bio-inspired mechanical design of a tendon-driven dexterous prosthetic hand. Conference proceedings : Annual International Conference of the IEEE Engineering in Medicine and Biology Society 2010:499-502.

Craig A (1867) The Book of the Hand: Or the Science of Modern Palmistry, Chiefly According to the Systems of D'Arpentigny and Desbarrolles. With Some Account of the Gipsies: S. Low and Marston. 
Culham JC, Danckert SL, DeSouza JF, Gati JS, Menon RS, Goodale MA (2003) Visually guided grasping produces fMRI activation in dorsal but not ventral stream brain areas. Experimental brain research 153:180-189.

Davare M, Kraskov A, Rothwell JC, Lemon RN (2011) Interactions between areas of the cortical grasping network. Current opinion in neurobiology 21:565-570.

Delp SL, Anderson FC, Arnold AS, Loan P, Habib A, John CT, Guendelman E, Thelen DG (2007) OpenSim: open-source software to create and analyze dynamic simulations of movement. IEEE transactions on biomedical engineering 54:1940-1950.

DiCarlo JJ, Zoccolan D, Rust NC (2012) How does the brain solve visual object recognition? Neuron 73:415-434.

Diez PF, Torres Muller SM, Mut VA, Laciar E, Avila E, Bastos-Filho TF, Sarcinelli-Filho M (2013) Commanding a robotic wheelchair with a high-frequency steady-state visual evoked potential based brain-computer interface. Medical engineering and physics 35:1155-1164.

Dipietro L, Sabatini AM, Dario P (2003) Evaluation of an instrumented glove for handmovement acquisition. Journal of Rehabilitation Research and Development 40:179-189.

Dum RP, Strick PL (1991) The origin of corticospinal projections from the premotor areas in the frontal lobe. The Journal of Neuroscience 11:667-689.

Dum RP, Strick PL (2005) Frontal lobe inputs to the digit representations of the motor areas on the lateral surface of the hemisphere. The Journal of Neuroscience 25:1375-1386.

Fagg AH, Arbib MA (1998) Modeling parietal-premotor interactions in primate control of grasping. Neural networks 11:1277-1303.

Fattori P, Breveglieri R, Raos V, Bosco A, Galletti C (2012) Vision for action in the macaque medial posterior parietal cortex. The Journal of Neuroscience 32:32213234.

Felleman DJ, Van Essen DC (1991) Distributed Hierarchical Processing in the Primate Cerebral Cortex. Cerebral Cortex 1:1-47.

Fluet MC, Baumann MA, Scherberger H (2010) Context-specific grasp movement representation in macaque ventral premotor cortex. The Journal of Neuroscience 30:15175-15184.

Fogassi L, Gallese V, Buccino G, Craighero L, Fadiga L, Rizzolatti G (2001) Cortical mechanism for the visual guidance of hand grasping movements in the monkey: A reversible inactivation study. Brain 124:571-586.

Gallese V, Murata A, Kaseda M, Niki N, Sakata H (1994) Deficit of hand preshaping after muscimol injection in monkey parietal cortex. Neuroreport 5:1525-1529.

Gentner R, Classen J (2009) Development and evaluation of a low-cost sensor glove for assessment of human finger movements in neurophysiological settings. Journal of Neuroscience Methods 178:138-147.

Georgopoulos AP, Schwartz AB, Kettner RE (1986) Neuronal population coding of movement direction. Science 233:1416-1419.

Gilja V, Chestek CA, Diester I, Henderson JM, Deisseroth K, Shenoy KV (2011) Challenges and opportunities for next-generation intracortically based neural prostheses. IEEE Transactions on Biomedical Engineering 58:1891-1899.

Godschalk M, Mitz AR, van Duin B, van der Burg H (1995) Somatotopy of monkey premotor cortex examined with microstimulation. Neuroscience research 23:269-279.

Goodale MA, Meenan JP, Bulthoff HH, Nicolle DA, Murphy KJ, Racicot CI (1994) Separate neural pathways for the visual analysis of object shape in perception and prehension. Current biology 4:604-610.

Goodale MA, Milner AD (1992) Separate visual pathways for perception and action. Trends in neurosciences 15:20-25. 
Goodale MA, Milner AD, Jakobson LS, Carey DP (1991) A Neurological Dissociation between Perceiving Objects and Grasping Them. Nature 349:154-156.

Graziano M (2006) The organization of behavioral repertoire in motor cortex. Annual Review of Neuroscience 29:105-134.

Graziano MS, Taylor CS, Moore T (2002) Complex movements evoked by microstimulation of precentral cortex. Neuron 34:841-851.

Harvill YL, Zimmermann TG, Grimaud JG (1992) Motion sensor wich produces an asymmetrical signal in response to symmetrical movement. (Patent, U. S., ed), p 13 USA: VPL Research Inc., Redwood City, Calif.

Hatsopoulos NG, Donoghue JP (2009) The science of neural interface systems. Annual review of neuroscience 32:249-266.

Hepp-Reymond MC, Husler EJ, Maier MA, Ql HX (1994) Force-related neuronal activity in two regions of the primate ventral premotor cortex. Canadian journal of physiology and pharmacology 72:571-579.

Hochberg LR, Bacher D, Jarosiewicz B, Masse NY, Simeral JD, Vogel J, Haddadin S, Liu J, Cash SS, van der Smagt P, Donoghue JP (2012) Reach and grasp by people with tetraplegia using a neurally controlled robotic arm. Nature 485:372-375.

Hochberg LR, Donoghue JP (2006) Sensors for brain-computer interfaces. IEEE engineering in medicine and biology magazine 25:32-38.

Holzbaur KR, Murray WM, Delp SL (2005) A model of the upper extremity for simulating musculoskeletal surgery and analyzing neuromuscular control. Annals of biomedical engineering 33:829-840.

Hubel DH, Wiesel TN (1968) Receptive Fields and Functional Architecture of Monkey Striate Cortex. Journal of Physiology 195:215-243.

James TW, Culham J, Humphrey GK, Milner AD, Goodale MA (2003) Ventral occipital lesions impair object recognition but not object-directed grasping: an fMRI study. Brain : a journal of neurology 126:2463-2475.

Jeannerod M, Arbib MA, Rizzolatti G, Sakata H (1995) Grasping objects: the cortical mechanisms of visuomotor transformation. Trends in neurosciences 18:314320.

Kakei S, Hoffman DS, Strick PL (1999) Muscle and movement representations in the primary motor cortex. Science 285:2136-2139.

Kandel ER, Schwartz JH, Jessell TM (2000) Principles of neural science: McGraw-Hill New York.

Kim SP, Simeral JD, Hochberg LR, Donoghue JP, Black MJ (2008) Neural control of computer cursor velocity by decoding motor cortical spiking activity in humans with tetraplegia. Journal of neural engineering 5:455-476.

Kim SP, Simeral JD, Hochberg LR, Donoghue JP, Friehs GM, Black MJ (2011) Point-andclick cursor control with an intracortical neural interface system by humans with tetraplegia. IEEE transactions on neural systems and rehabilitation engineering 19:193-203.

Kolb B, Whishaw IQ (2002) Brain and Behavior: Worth Publishers Inc.

Komatsu H, Ideura Y (1993) Relationships between Color, Shape, and Pattern Selectivities of Neurons in the Inferior Temporal Cortex of the Monkey. Journal of Neurophysiology 70:677-694.

Konen CS, Kastner S (2008) Two hierarchically organized neural systems for object information in human visual cortex. Nature neuroscience 11:224-231.

Kraskov A, Prabhu G, Quallo MM, Lemon RN, Brochier T (2011) Ventral premotor-motor cortex interactions in the macaque monkey during grasp: response of single neurons to intracortical microstimulation. The Journal of Neuroscience 31:88128821.

Kuffler SW (1953) Discharge Patterns and Functional Organization of Mammalian Retina. Journal of Neurophysiology 16:37-68. 
Lehmann SJ, Scherberger H (2013) Reach and gaze representations in macaque parietal and premotor grasp areas. The Journal of Neuroscience 33:7038-7049.

Li W, Gilbert CD (2002) Global contour saliency and local colinear interactions. Journal of Neurophysiology 88:2846-2856.

Li W, Piech V, Gilbert CD (2006) Contour saliency in primary visual cortex. Neuron 50:951-962.

Logothetis NK, Pauls J, Poggio T (1995) Shape representation in the inferior temporal cortex of monkeys. Current biology 5:552-563.

Logothetis NK, Sheinberg DL (1996) Visual object recognition. Annual review of neuroscience 19:577-621.

Luppino G, Murata A, Govoni P, Matelli M (1999) Largely segregated parietofrontal connections linking rostral intraparietal cortex (areas AIP and VIP) and the ventral premotor cortex (areas F5 and F4). Experimental brain research 128:181-187.

Maunsell JH, Van Essen DC (1983) Functional properties of neurons in middle temporal visual area of the macaque monkey. I. Selectivity for stimulus direction, speed, and orientation. Journal of Neurophysiology 49:1127-1147.

Merigan WH, Katz LM, Maunsell JH (1991) The effects of parvocellular lateral geniculate lesions on the acuity and contrast sensitivity of macaque monkeys. The Journal of Neuroscience 11:994-1001.

Merigan WH, Maunsell JH (1990) Macaque vision after magnocellular lateral geniculate lesions. Visual neuroscience 5:347-352.

Mountcastle VB, Lynch JC, Georgopoulos A, Sakata H, Acuna C (1975) Posterior parietal association cortex of the monkey: command functions for operations within extrapersonal space. Journal of Neurophysiology 38:871-908.

Murata A, Fadiga L, Fogassi L, Gallese V, Raos V, Rizzolatti G (1997) Object representation in the ventral premotor cortex (area F5) of the monkey. Journal of Neurophysiology 78:2226-2230.

Murata A, Gallese V, Luppino G, Kaseda M, Sakata H (2000) Selectivity for the shape, size, and orientation of objects for grasping in neurons of monkey parietal area AIP. Journal of Neurophysiology 83:2580-2601.

Musallam S, Corneil BD, Greger B, Scherberger H, Andersen RA (2004) Cognitive control signals for neural prosthetics. Science 305:258-262.

Nakamura H, Kuroda T, Wakita M, Kusunoki M, Kato A, Mikami A, Sakata H, Itoh K (2001) From three-dimensional space vision to prehensile hand movements: the lateral intraparietal area links the area V3A and the anterior intraparietal area in macaques. The Journal of Neuroscience 21:8174-8187.

Napier JR (1956) The Prehensile Movements of the Human Hand. Journal of Bone and Joint Surgery-British Volume 38:902-913.

Nelissen K, Vanduffel W (2011) Grasping-related functional magnetic resonance imaging brain responses in the macaque monkey. The Journal of Neuroscience 31:82208229.

Norman J (2002) Two visual systems and two theories of perception: An attempt to reconcile the constructivist and ecological approaches. The behavioral and brain sciences 25:73-144.

O'Doherty JE, Lebedev MA, Hanson TL, Fitzsimmons NA, Nicolelis MA (2009) A brainmachine interface instructed by direct intracortical microstimulation. Frontiers in integrative neuroscience 3:20.

O'Doherty JE, Lebedev MA, Ifft PJ, Zhuang KZ, Shokur S, Bleuler H, Nicolelis MA (2011) Active tactile exploration using a brain-machine-brain interface. Nature 479:228-231.

Overduin SA, Zaheer F, Bizzi E, d'Avella A (2010) An instrumented glove for small primates. Journal of Neuroscience Methods 187:100-104. 
Palmeri TJ, Gauthier I (2004) Visual object understanding. Nature Reviews Neuroscience 5:291-U217.

Pesaran B, Nelson MJ, Andersen RA (2006) Dorsal premotor neurons encode the relative position of the hand, eye, and goal during reach planning. Neuron 51:125-134.

Raos V, Umilta MA, Murata A, Fogassi L, Gallese V (2006) Functional properties of grasping-related neurons in the ventral premotor area F5 of the macaque monkey. Journal of Neurophysiology 95:709-729.

Rathelot JA, Strick PL (2009) Subdivisions of primary motor cortex based on corticomotoneuronal cells. Proceedings of the National Academy of Sciences of the United States of America 106:918-923.

Reed CL, Shoham S, Halgren E (2004) Neural substrates of tactile object recognition: an fMRI study. Human brain mapping 21:236-246.

Rizzolatti G, Camarda R, Fogassi L, Gentilucci M, Luppino G, Matelli M (1988) Functional organization of inferior area 6 in the macaque monkey. II. Area F5 and the control of distal movements. Experimental brain research 71:491-507.

Rizzolatti G, Fogassi L, Gallese V (2001) Neurophysiological mechanisms underlying the understanding and imitation of action. Nature Reviews Neuroscience 2:661-670.

Rizzolatti G, Luppino G (2001) The cortical motor system. Neuron 31:889-901.

Romero MC, Pani P, Janssen P (2014) Coding of shape features in the macaque anterior intraparietal area. The Journal of Neuroscience 34:4006-4021.

Sakata H, Taira M, Kusunoki M, Murata A, Tanaka Y (1997) The TINS lecture - The parietal association cortex in depth perception and visual control of hand action. Trends in neurosciences 20:350-357.

Sakata H, Taira M, Murata A, Mine S (1995) Neural mechanisms of visual guidance of hand action in the parietal cortex of the monkey. Cerebral cortex 5:429-438.

Schaffelhofer S, Agudelo-Toro A, Scherberger H (in press) Decoding a wide range of hand configurations from macaque Motor, Premotor, and Parietal Cortices. The Journal of Neuroscience.

Schaffelhofer S, Sartori M, Scherberger H, Farina D (2014) Musculoskeletal Representation of a Large Repertoire of Hand Grasping Actions in Primates. IEEE Transactions on Neural Systems and Rehabilitation Engineering. Advance online publication Retrieved November 25, 2014 doi:101109/TNSRE20142364776.

Schaffelhofer S, Scherberger H (2012) A new method of accurate hand- and armtracking for small primates. Journal of neural engineering 9:026025.

Scherberger H (2009) Neural control of motor prostheses. Current opinion in neurobiology 19:629-633.

Schieber MH (2001) Constraints on somatotopic organization in the primary motor cortex. Journal of Neurophysiology 86:2125-2143.

Schieber MH, Hibbard LS (1993) How somatotopic is the motor cortex hand area? Science 261:489-492.

Schieber MH, Poliakov AV (1998) Partial inactivation of the primary motor cortex hand area: effects on individuated finger movements. The Journal of Neuroscience 18:9038-9054.

Schieber MH, Santello M (2004) Hand function: peripheral and central constraints on performance. Journal of applied physiology 96:2293-2300.

Schmidlin E, Brochier T, Maier MA, Kirkwood PA, Lemon RN (2008) Pronounced reduction of digit motor responses evoked from macaque ventral premotor cortex after reversible inactivation of the primary motor cortex hand area. The Journal of Neuroscience 28:5772-5783.

Scott SH (2004) Optimal feedback control and the neural basis of volitional motor control. Nature Reviews Neuroscience 5:532-546.

Simone LK, Sundarrajan N, Luo X, Jia Y, Kamper DG (2007) A low cost instrumented glove for extended monitoring and functional hand assessment. Journal of Neuroscience Methods 160:335-348. 
Subasi E, Townsend B, Scherberger H (2010) In search of more robust decoding algorithms for neural prostheses, a data driven approach. In: Engineering in Medicine and Biology Society (EMBC), 2010 Annual International Conference of the IEEE, pp 4172-4175: IEEE.

Taira M, Mine S, Georgopoulos AP, Murata A, Sakata H (1990) Parietal cortex neurons of the monkey related to the visual guidance of hand movement. Experimental brain research 83:29-36.

Tanaka K (1996) Inferotemporal cortex and object vision. Annual review of neuroscience 19:109-139.

Theys T, Pani P, van Loon J, Goffin J, Janssen P (2012a) Selectivity for three-dimensional shape and grasping-related activity in the macaque ventral premotor cortex. The Journal of Neuroscience 32:12038-12050.

Theys T, Srivastava S, van Loon J, Goffin J, Janssen P (2012b) Selectivity for threedimensional contours and surfaces in the anterior intraparietal area. Journal of Neurophysiology 107:995-1008.

Thorpe S, Fize D, Marlot C (1996) Speed of processing in the human visual system. Nature 381:520-522.

Townsend BR, Subasi E, Scherberger H (2011) Grasp movement decoding from premotor and parietal cortex. The Journal of Neuroscience 31:14386-14398.

Townsend G, LaPallo BK, Boulay CB, Krusienski DJ, Frye GE, Hauser CK, Schwartz NE, Vaughan TM, Wolpaw JR, Sellers EW (2010) A novel P300-based brain-computer interface stimulus presentation paradigm: moving beyond rows and columns. Clinical neurophysiology 121:1109-1120.

Umilta MA, Brochier T, Spinks RL, Lemon RN (2007) Simultaneous recording of macaque premotor and primary motor cortex neuronal populations reveals different functional contributions to visuomotor grasp. Journal of Neurophysiology 98:488-501.

Vargas-Irwin CE, Shakhnarovich G, Yadollahpour P, Mislow JM, Black MJ, Donoghue JP (2010) Decoding complete reach and grasp actions from local primary motor cortex populations. The Journal of Neuroscience 30:9659-9669.

Velliste M, Perel S, Spalding MC, Whitford AS, Schwartz AB (2008) Cortical control of a prosthetic arm for self-feeding. Nature 453:1098-1101.

Zhuang J, Truccolo W, Vargas-Irwin C, Donoghue JP (2010) Decoding 3-D reach and grasp kinematics from high-frequency local field potentials in primate primary motor cortex. IEEE Transactions on Biomedical Engineering 57:1774-1784. 




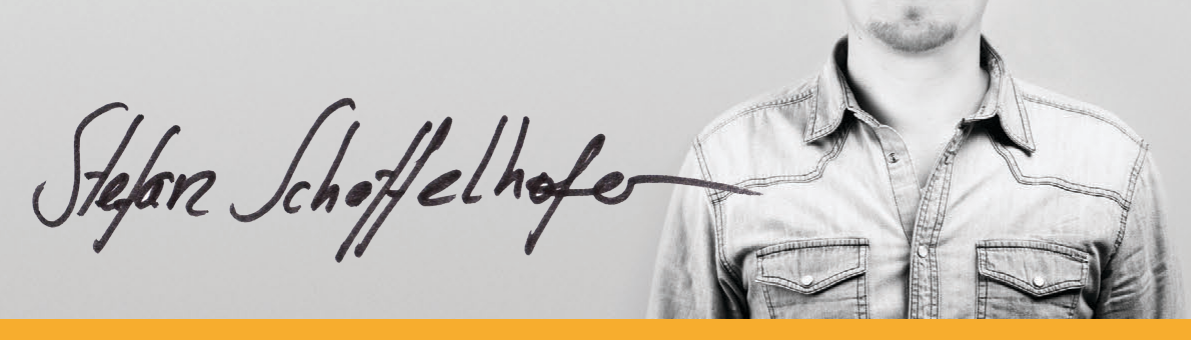

PERSONAL INFORMATION

Name:

Stefan Schaffelhofer

Address:

Zimmermannstraße 60, 37075 Göttingen, Germany

Telephone:

+491745363051

E-mail:

schaffelhofer@me.com

Nationality:

Austrian

Date of birth:

27.10.1983

Gender:

Male

\section{EDUCTATION / STUDY}

2009-2014:

Ph.D. student at the Georg August Universität Göttingen, Research group of Professor Dr. Hansjörg Scherberger

Degree: Dr.rer.nat, predicate "summa cum laude"

Ph.D. Thesis: „From vision to action:The neural representation of hand actions in macaque hand grasping areas AIP, F5 and M1"

2004-2008:

Diploma Study of Medical Engineering at the University of Applied

Sciences Upper Austria, Linz

Degree:Dipl. Ing. (FH), predicate „Mit Auszeichnung bestanden“

("excellent").

Diploma Thesis: „Online position reconstruction of rats by the use of neural spike information from hippocampal place cells and grid cells" in cooperation with g.tec

1998-2003:

Polytechnikum for Electrical Engineering Leonding (Höhere Technische Bundeslehranstalt Leonding)

\section{Degree: Ing.}

Diploma Thesis: „A contactless gear tooth sensor realized with Hall effect sensors" 
German Primate Center GmbH, Research group Neurobiology (since August 2014)

Position: Postdoctoral Research Scientist

\section{German Primate Center GmbH, Research group Neurobiology (November 2009 - July 2014)}

Position: Ph.D. student in Neuroscience enrolled at the Georg August Universität Göttingen and member of the Göttinger Graduate School for Neuroscience and Molecular Biology.

Research projects:

- Development of a hand and finger-tracking system based on magnetic sensors for humans and non-human primates

- Development of a musculoskeletal model for describing hand and arm motion in non-human primates

- Decoding grip types from macaque motor (M1), pre-motor (F5) and parietal cortex (AIP) for neuro prosthetics hand control

- Object or grip type representation? A comparative population study of hand grasping areas AIP, F5, and M1.

- The representation of others' hand actions in AIP.

\section{g.tec GmbH, Department for Research \& Development (August 2008 - August 2009)}

Position: Research and Software Development Engineer

Engineering projects:

- Development of a real-time recording software for extracellular action potential acquisition

- Development of a software package for place- and grid cell analysis

Research Projects:

- In-house project manager of EU-project "Synthetic Forager" (funded by 7th Framework Program, project reference: 217148 )

- Co-operation for EU-project „Presenccia“ (funded by 6th Framework Program, project reference: 27731)

- Executive engineer for the art project:„Multimodal Brain Orchestra“ presented at the FET 2009 in Prague.

g.tec GmbH, Practice training (July 2007 - December 2007)

Development of a grid-cell analysis toolbox for Matlab.

Schaffelhofer CATV, part-time job during studies (October 2004 - June 2007)

Maintenance of Cabel-TV monitoring systems, network administration;

Österreichisches Bundesheer, basic military service (January 2004 - August 2004)

Infantry battalion, Amstetten, Austria

Lindsay Electronics Ltd. Canada, internship (October 2003-December 2003)

Final tests of Cabel-TV amplifiers, Design of analogue filters

Leotec GmbH, high-school diploma thesis (September 2002- June 2003)

Development of a gear-tooth sensor based on Hall effect sensors

A.Haberkorn \& CO. GmbH, internship (Summer 2002)

Quality management 
Schaffelhofer S., Agudelo-Toro A., Scherberger H., Decoding a wide range of hand configurations from macaque motor, premotor and parietal cortex. J Neurosci. In press.

Schaffelhofer S.*, Sartori M.*, Scherberger H., Farina D., Musculoskeletal Representation of a Large Repertoire of Hand Grasping Actions in Primates. IEEE Trans Neural Syst Rehabil Eng. 2014.

Schaffelhofer S.* \& Scherberger $\mathrm{H}^{*}$, Modelling of hand and arm position and orientation. Patent No. EP2418562 B1, 2013.

Schaffelhofer S. \& Scherberger H., A new method of accurate hand- and arm-tracking for small primates. Journal of neural engineering. 2012.

Guger C., Gener T., Pennartz C. M., Brotons-Mas J.R., Edlinger G., Bermudez I. S., Verschure P., Schaffelhofer S., Sanchez-Vives M.V., Real time position reconstruction with hippocampal place cells. Frontiers in neuroscience. 2011.

\section{PEER REVIEWED PROCEEDINGS}

Schaffelhofer S., Guger C., Brotons-Mas J.R., Sanchez-Vives M.V.\& Gener T., Position reconstruction of rats by the use of neural spike information from hippocampal place cells. European Future Technologies Conference, Prague, Czech Republic, April 21-23, 2009.

Schaffelhofer S., Guger C., Sanchez-Vives M.V., Brotons-Mas J. R. \& Gener T., Position decoding of hippocampal place cells. IFMBE Proceedings, World Congress on Medical Physics and Biomedical Engineering. Munich, Germany, September 7-12, 2009.

\section{UNDER REVIEW / IN PREPARATION}

Schaffelhofer S. \& Scherberger H., From vision to action: A comparative population study of hand grasping areas AIP, F5, and M1. Prepared for submission. 2014.

Menz. V.K.* , Schaffelhofer S.* , Scherberger H. Decoding of hand kinematics from parietal, premotor, and motor cortex. Prepared for submission. 2014.

\section{ABSTRACTS}

Schaffelhofer S. \& Scherberger H., Object or grip type representation? A comparative population study of hand grasping areas AIP, F5, and M1. Neuroscience. New Orleans, USA, October 13-17, 2012.

Schaffelhofer S. \& Scherberger H., Decoding grip types from macaque motor (M1), premotor (F5) and parietal cortex (AIP). Neuroscience. New Orleans, USA, October 13-17, 2012.

Menz K.*, Schaffelhofer S.* \& Scherberger H., Detection and decoding of continuous hand and arm kinematics from primate motor, premotor, and parietal cortex. Neuroscience, New Orelans, USA, October 13-17, 2012.

Schaffelhofer S. \& Scherberger H., A new hand and finger tracking system for non-human primates based on magnetic sensors. Neuroscience. San Diego, USA, Nov. 13-17, 2010.

Schaffelhofer S., Guger C., Sanchez-Vives M. V., Gener T. \& Brotons-Mas J. R., Online reconstruction of rat's position based on firing information from hippocampal place cells. Neuroscience. Chicago, USA, October 17-21, 2009. 


\section{TALKS}

From Vision to Action: Hand representations in macaque grasping areas AIP, F5, and M1. Neuroscience. Washington D.C., USA, Nov. 15-19, 2014.

Object or grip type representation? A comparative population study of hand grasping areas AIP, F5, and M1. 10th Göttinger Meeting of the German Neuroscience Society, Göttingen, Germany, March 13-16, 2013.

Online position reconstruction of rats by the use of neural spike information from hippocampal place cells, Biosignal 2008, Brno, Czech Republic, June 29-30, 2008.

\section{AWARDS}

"Förderpreis des Deutschen Primatenzentrums" for an outstanding research with primates. 2014

"CSF junior award" for best scientific contribution. Hand brain and technology 2014. Ascona, Switzerland.

\section{MEDIA}

TV-Interview: Simmons D., Can robots ever be like humans?, BBC, 2009.

Online magazine article: Krüger A., Rollstuhl mit Gedanken steuern, ZDF heute.de, 2009.

Book article: Chorost M.,World wide mind:The coming integration of humanity, machines, and the Internet. Free Press, 2011.

\section{TUTORING EXPERIENCE}

Thesis supervision: Kratzenberg A., Categorization of single neurons in macaque brain areas AIP, F5, and M1. Bachelor thesis. 2012.

Thesis supervision: Minarik T., Analysis of neural activity from hippocampus and cerebellum in rats. Master thesis. 2009.

Organization of lecture series "Motor Systems", held by Prof. Hansjörg Scherberger at the University of Göttingen. 2011.

\section{CAPABILITIES}

Languages:

Software Engineering:

Hardware Engineering:

Operating Systems:

Computer skills:

Driving licence:
German (native), English (fluent)

Matlab, Simulink, C++, Labview, OpenSim;

Eagle Layout Editor, AutoCAD

Windows, Mac OS, Mac OS Server

MS Word, Excel, Adobe Illustrator, Photoshop, Finalcut

European class $A, B$;

\section{PERSONAL INTERESTS}

Diving, football and tactics, race simulations, and family.

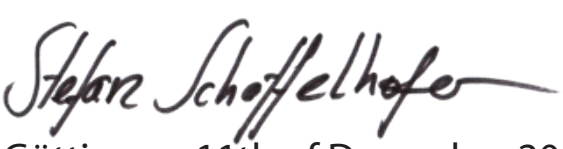

Göttingen, 11 th of December 2014 